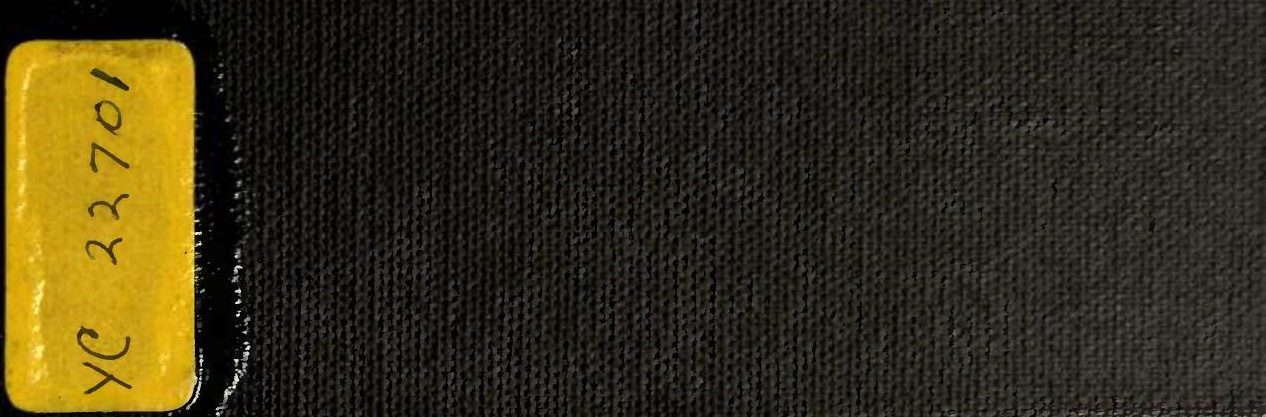





A

\section{COURSE OF LECTURES}

on

\section{NATURAL PHILOSOPHY,}

$\& c$.

VOLUME II.—PLATES. 


\section{COURSE OF LECTURES}

ON

\section{NATURAL PHILOSOPHY \\ AND THE}

\section{MECHANICAL ARTS.}

BY THOMAS YOUNG, M.D.,

FOR. SEC. R.S., FOREIGN A SSOCIATE OF THE ROYAL INSTITUTE OF FRANCE, MEMBER OF EMMANUEL COLLege, CAMBRIDGe, AND PROFEgror OF NATURAL PHILOSOPHY IN THE ROYAL INSTITUTION OF GREAT BRITAIN.

A NEW EDITION, WITH REFERENCES AND NOTES, BY THE

REV. P. KELLAND, M.A., F.R.S., Lond. \& Epinb., LATE FELLOW OF QUEN'S COLLEGE, CAMBRIDGe; PROFESSOR OF MATHEMATICS, ETC. IN THE UNIVERITY OF EDINBURGH.

Ellustrated by fumerous Engrabings on Copper.

IN TWO VOLUMES.

VOLUME II.—PLATES.

$$
\text { L O N D O N : }
$$

PRINTED FOR TAYLOR AND WALTON, UPPER GOWER STREET. 
Printed by J. \& H. COX, BROTHERS (LATE COX \& SONS), $74 \&$ 75, Great Queen Street, Lincoln's-Inn Fields. 
47
1845
$V .2$

\section{DESCRIPTION OF PLATES.}




\section{DESCRIPTION OF PLATES.}

\section{P L A T E I.}

Fig. 1. The point $\mathbf{A}$ being supposed to move in a right line to $\mathbf{B}, \mathbf{A} \mathbf{B}$ is the direction of its motion. P. 15.

Fig. 2. The lines A B, B C, C D, are the successive directions of the point A, moving from $A$ to $D$ in the figure $A B C D . P .15$.

Fig. 3. The tangent $\mathrm{A} B$ is the direction of the motion of the point $\mathbf{C}$, moving in the curve C D, when it arrives at E. P. 15.

Fig. 4. The square A B, moving on the board C D, so that the points E, F, describe the parallel lines E G, F H, with equal velocities, the plane A E F B is in rectilinear motion with respect to the surface C D. P. 18.

Fig. 5. The cycloid A B C, and the trochoid DEF are the results of the rotatory motion of the points B and E round the centre of the wheel, combined with the progressive motion of the wheel along the base A C. P. 18, 34 .

Fig. 6. A B is a fixed bar, C D an arm which slides on it, E C F a thread passing round the pulley at $\mathrm{C}$, and either fixed to the pin on the slider $\mathrm{F}$, or passed over the pulley $G$, and fixed again at $H$. The arm turns round the same axis that carries the pulley at $\mathrm{C}$, and may be fixed by means of the screw which is cut on the axis, while two other screws keep it steady by pressing on the slider below it. The point I describes, by its compound motion, the oblique line K I. P. 18.

Fig. 7. The diagonal A B of the parallelogram C D is the joint result of the motions represented by its sides A C, A D. P. 19.

Fig. 8. The line A B may be either simply drawn in the direction A B, or it may be traced by the equal motions A C and A D of the arm and its slider, or by the unequal motions $\mathrm{A}$ E and A F. P.'19.

Fig. 9. The body A, moving uniformly along the line A B, first approaches to the point $\mathrm{C}$, and then recedes from it, as if repelled. P. 21.

Fig. 10. When A B and A C approach each other, and coincide, the diagonal A D becomes equal to their sum. P. 23.

Fig. 11. Atwood's machine. The boxes A, B, containing equal weights, are connected by the thread A C B, passing over the pulley C, which is supported either on friction wheels, or by the points of screws, one of which is seen at D. The box $A$ is made to descend either by a flat weight placed on it, or by the bar E, which is intercepted by the ring $\mathbf{F}$, and the box continues to descend till it strikes the stage $G$; the space being measured on the scale $H I$, and the time by the pendulum $K$, which may be kept in motion by a clock scapement with a weight. The machine is levelled by the screws L, M. P. 23, 41 .

Fig. 12. The time of the descent of a falling body being represented by any portion $\mathrm{A} \mathrm{B}$ of the base of a triangle, the velocity will be proportional to $\mathrm{BC}$, which is equal to $\mathrm{AB}$, and the space described during the time D E, supposed infinitely short, will be proportional to the area D E F G, which is expressed by the product of $\mathrm{B} \mathrm{C}$ and D E ; consequently the whole area A E F will represent the space described in the time AE, and A H I the space described in the time $A \mathrm{H}$; but A H I is half of the square $H K$, and A E F of EL: the space is therefore always as the square of the time, and is equal to half the space which would be described in the same time with the final velocity. P. 24.

Fig. 13. The whirling table. The arms A B, C D, are made to revolve on the axes $\mathrm{E} \mathrm{F,} \mathrm{G} \mathrm{H} \mathrm{by} \mathrm{the} \mathrm{string} \mathrm{passing} \mathrm{over} \mathrm{the} \mathrm{wheel} \mathrm{I,} \mathrm{the} \mathrm{upper} \mathrm{or} \mathrm{under} \mathrm{pulley} \mathrm{of}$ either axis being employed at pleasure : the stages $\mathrm{K}, \mathrm{L}$, with their weights, are placed at certain distances from the centre, by means of the racks or teeth below them ; they move along the arms by means of friction wheels resting on wires, and they raise the weights $\mathbf{M}, \mathbf{N}$, by means of threads passing each over two pullies. P. 27 .

Fig. 14. If a body revolving in a curve A B C, by means of a force directed to $\mathrm{D}$, describe the portions $\mathrm{A} \mathrm{E}, \mathrm{BF}, \mathrm{CG}$ in equal times, the areas A DE, B D F, CD G, will be equal, and the velocities in A, B, and $G$, will be inversely as the perpendiculars D H, D I, and D K. P. 28. 


\section{P L A T E II.}

Fig. 15. The ball $A$, revolving round the point $B$, and being drawn towards it by means of the thread B C, with a force variable at pleasure, its velocity may be observed to vary according to its distance from the point B. P. 29.

Fig. 16. The curve A B C D E is an ellipsis ; F and G are its foci, A D its greater axis, and $\mathrm{C} \mathrm{E}$ its lesser axis. P. 29.

Fig. 17. The horizontal range, A B, of a body projected at an elevation of $45^{\circ}$, is greater than $\mathbf{A C}$ or $\mathbf{A ~ D , ~ t h e ~ r a n g e s ~ o f ~ b o d i e s ~ p r o j e c t e d ~ w i t h ~ t h e ~ s a m e ~ v e l o c i t y ~ a t ~}$ a greater or less elevation. If the parallel lines $\mathrm{E} \mathrm{F,} \mathrm{G} \mathrm{H,} \mathrm{be} \mathrm{always} \mathrm{as} \mathrm{the} \mathrm{squares}$ of A E, A G, the curve A F H will be a parabola ; and such is the path of a projectile. P. 31 .

Fig. 18. The path of a ball moving swiftly through the atmosphere nearly resembles the curve A B. P. 31 .

Fig. 19. The ball A, having descended along the groove A B, describes the parabola $\mathrm{B} \mathrm{C}$, passing through the rings $\mathrm{D}, \mathrm{E}$. P. 31 .

Fig. 20. The cylinder A, loaded at the axis, descends along an inclined plane more rapidly than the cylinder $B$, loaded with an equal weight at the circumference. P. 33.

Fig. 21. The balls A, B, C, descend along the planes A D, B E, C F, of equal height, in times proportional to their lengths. The upper surfaces of the slips AD, B E, C F, are slightly grooved. P. 33.

Fig. 22. The balls A, B, C, descend in equal times along the chords A D, B D, C D. P. 33 .

Fig. 23. The same ball, descending from equal heights, at A, B, or C, by different paths, will rise to the same height at $D$ on the opposite side of $E$. P. 34 .

Fig. 24. The thread A B, playing between the cycloidal cheeks A C, A D, describes the cycloid C E D ; and the balls B, F, descending from any two points of the curve, will meet at $\mathrm{E}$, in the same time that the ball $\mathrm{G}$ falls from a point nearly $\frac{1}{4}$ of $\mathrm{A} \mathrm{E}$ above $\mathrm{A}$. The space described by the pendulum in descending is always proportional to the height $\mathrm{HI}$, to which a body setting out from $\mathrm{E}$, and revolving uniformly in a circle, will rise in the same time. The circle E I lies without the cycloid C E D, and is somewhat less inclined to the horizon at equal distances from E. P. 35 .

Fig. 25. The ball A, descending from $\mathbf{B}$ in the curve $\mathbf{B} \mathbf{A}$, arrives at $\mathbf{C}$ before the ball $\mathrm{D}$, moving in a right line on the plane B C. P. 36 .

Fig. 26. The balls A, B, C, being made to revolve by means of the whirling table, they are always found in the same horizontal plane. The joint connecting them with the axis is represented at $\mathrm{D}$, as seen from above. P. 36 .

Fig. 27. The equal vibrations, represented by A B, C D, compose, when united, the circular revolution, A E B : the unequal vibrations A B, F G, compose the ellipsis $\mathrm{A} \mathrm{H} \mathrm{B}$; the place of the body being always ascertained by combining the versed sines of two circular arcs increasing uniformly. P. 37.

Fig. 28. The balls A, B, as their revolution becomes more rapid, fly out, and the point $\mathrm{C}$ is depressed. P. 37 .

Fig. 29. The mass of the body A being 1, and that of B 2, and A C being twice $\mathrm{B} \mathrm{C}, \mathrm{C}$ is the centre of inertia [gravity]. P. 40.

Fig. 30. The balls $A$ and $B$ are suspended by long threads, which allow them to move in the arcs A C, B D ; the ball A is perforated in a horizontal direction, and contains a spiral spring, which is confined by the thread $\mathbf{E}$, and being set at liberty by burning this thread, strikes the ball $\mathrm{B}$, so as to cause each of the balls to move through an arc, of which the chord is proportional to the weight of the other ball. P. 40 . 


\section{P L A T E III.}

Fig. 31. The centre of inertia of the bodies A, B, C, D, may be determined, either by finding $\mathbf{E}$ the centre of inertia of $\mathrm{A}$ and $\mathrm{B}$, and supposing a body equal to their sum to be placed in it, then determining $F$ from $\mathbf{E}$ and $\mathbf{C}$; and $\mathbf{G}$, the point required, from $F$ and $D$; or by finding first $H$ and $I$ from $A, C, B, D$, taken in pairs, and dividing $\mathrm{H} I$ in due proportion in the same point G. P. 41 .

Fig. 32. The point A being the centre of inertia of the bodies B, C, D, E, the products obtained by multiplying $\mathrm{B}$ by $\mathrm{BF}, \mathrm{C}$ by $\mathrm{C} \mathrm{G}, \mathrm{D}$ by $\mathrm{D} \mathrm{H}$, and $\mathrm{E}$ by $\mathrm{E} \mathrm{I}$, are equal, when added together, to the product of the masses of all the bodies by the distance A K ; all the lines drawn to the plane F I being parallel. P. 42.

Fig. 33. The weights A B C will remain at rest when they are in the same proportion to each other as the respective sides of the triangle DE F ; D F being parallel to E G. P. 47.

Fig. 34. The bodies A, B, remain in equilibrium when their centre of inertia $\mathrm{C}$ is immediately below the point of suspension $D$. P. 47 .

Fig. 35. The system of bodies A, B, C, is at rest when the centre of inertia D is immediately below the point of suspension E. P. 47 .

Fig. 36. The bodies A, B, remain at rest when the centre of inertia $\mathbf{C}$ is immediately above the point of support D. P. 47 .

Fig. 37. The bodies $\mathrm{A}, \mathrm{B}$, remain at rest when the centre of inertia $\mathbf{C}$ coincides with the fulcrum, or point of support. P. 47 .

Fig. 38. The irregular body A B remains at rest when the centre of inertia $\mathbf{C}$ is immediately below the point of suspension D. P. 47 .

Fig. 39. A being the centre of gravity of the board B, C, the point of suspension being $\mathrm{D}, \mathrm{E}$, or $\mathrm{F}$, the position of the vertical line will be $\mathrm{DA}, \mathrm{E} \mathrm{A}$, or $\mathrm{F} \mathrm{A}$. P. 47 .

Fig. 40. The equilibrium of the vessel $\mathrm{A}$ is stable, that of the vessel $\mathrm{B}$ tottering; the path of the centre of gravity having its concavity upwards in the first, and downwards in the second. P. 48.

Fig. 41. Paths of the centre of gravity of an oval. P. 48.

Fig. 42. Paths of the centre of gravity of a body resting on a sphere. P. 48.

Fig. 43. A, the path of the centre of gravity of a body standing on a flat basis ; B, the tottering equilibrium of the same body inclined. P. 48 .

Fig. 44. The effects of a certain inclination of a waggon, loaded with light and heavy materials, are represented at $A$ and $B$ respectively. P. 48.

Fig. 45. The suspension of a weight from a rod projecting over a table. P. 49 .

Fig. 46. A shows the path of the centre of gravity of a loaded cylinder on an inclined plane, $\mathrm{B}$ that of the centre of gravity of a double cone moving towards the more elevated end of a triangular surface. $\mathbf{C}$ is an elevation of the double cone. P. 49.

Fig. 47. A B is a lever of the first kind, the forces acting on different sides of the fulcrum $\mathrm{C} ; \mathrm{DE}$ of the second kind, the forces being applied at $\mathrm{D}$ and $\mathrm{F}$, on the same side of E. P. 50.

Fig. 48. A force applied at A may be held in equilibrium by a triple force, applied in the direction $\mathrm{B} \mathrm{C}$, either at $\mathrm{B}$ or at $\mathrm{C}$, or in a direction perpendicular to the arm $\mathrm{CD}$ at $\mathrm{E}, \mathrm{DE}$ and D B being each one third of A D. P. 51 .

Fig. 49. A force, acting at $\mathrm{A}$ on the lever $\mathrm{A} \mathrm{B}$, has a great mechanical advantage in turning the lever $\mathrm{C} \mathrm{D}$; but when the levers are in the position $\mathrm{B} \mathrm{E}, \mathrm{DF}$, the force acts with a similar disadvantage. P. 51 .

Fig. 50. The diameter of the cylinder $\mathbf{A}$ being three times as great as that of $\mathbf{B}$, the weight $C$, or an equivalent force applied to the winch $D$, will support a triple weight at E. P. 51 . 


\section{PLA T E IV.}

Fig. 51. The weight A, acting on the double cylinder B, supports the weight $\mathbf{C}$ by the pulley running in the angle of the rope D C E, which is wound on the larger cylinder at $\mathrm{D}$, while it is uncoiled from the smaller at $\mathbf{E}$, and the force is the same as if the weight $\mathbf{C}$ were attached to the line $\mathbf{C F}$, acting on the axis $\mathbf{F}$, of which the diameter is equal to the difference of the radii of the double cylinder. P. 52,158 .

Fig. 52. A single fixed pulley, supporting two equal weights. P. 52.

Fig. 53. A single moveable pulley, by means of which a weight supports another twice as great. P. 52.

Fig. 54. The arrangement of pullies in ships' tackles, with a force of six to one. P. 53.

Fig. 55. An arrangement of pullies in a vertical line, with a force of six to one. P. 53.

Fig. 56. Mr. Smeaton's blocks, giving a force of twenty to one, the rope being applied in the middle of the outer series, ana following the order of the figures from 1 to 21 . P. 53, 159.

Fig. 57. A system of pullies fixed on one axis in each block; having a power of 8 to 1 . P. 53.

Fig. 58. A system of pullies, each of which doubles the effect; having a power of 8 to 1. P. 53.

Fig. 59. A system of pullies with each rope fixed to the weight, having a force of 7 to 1. P. 53.

Fig. 60. Two systems of pullies, of the kind denominated Spanish bartons, in which two of the pullies are suspended by the same rope; the one has a power of 4 , the other of $5 . \quad$ P. 53.

Fig. 61. A. The depression of the middle weight being one third of its distance from the pullies, it sustains two equal weights, which are together three times as great as itself. B. The depression of the smaller weight being one fourth of its distance from the pulley, it supports a weight twice as great as itself. P. 53 .

Fig. 62. A joiner's saw, stretched by twisting a double cord, by means of a lever passing through it. P. 54.

Fig. 63. The weight A, resting on an inclined plane of which the height is to the oblique length as 3 to 5 , is sustained by a weight $B$ three fifths as great as itself; and if for the resistance of the plane we substitute the action of the weight $\mathbf{C}$, reduced to the direction A D perpendicular to the plane, this weight must be four fifths of the weight $\mathbf{A}$, the horizontal length of the wedge being four fifths of its oblique length. P. 54.

Fig. 64. The weights A, B, and C, acting, by means of threads passing over pullies, which are fixed to any required part of a horizontal table, on the rollers which press against the sides of a wedge, proportional in length to the respective weights, retain each other in equilibrium, when their directions meet in one point. In order that the threads may pass on each side of the wedge, it may be supported by three or more balls. P. 54 . 


\section{P L A T E V.}

Fig. 65. By means of the moveable inclined plane A B, of which the height A C is one third of the horizontal length B C, the weight D, acting horizontally, sustains a triple weight $\mathbf{E}$, acting in a vertical direction. P. 55 .

Fig. 66. A B being one fourth of B C, the rope A B must exert a force of tension equal to one fourth of the weight $\mathrm{C}$, in order to support it, supposing the surfaces to be without friction. But if the friction of the end of the beam $\mathbf{A} \mathbf{C}$ were equal to one fourth of the pressure, it would support the weight $\mathrm{C}$ without any other force, whatever might be its magnitude. P. 55.

Fig. 67. A B being half of $\mathrm{BC}$, or one fourth of $\mathrm{CD}$, the force extending the rope $\mathrm{C} \mathrm{D}$ each way is equal to the weight $\mathrm{E}$. P. 55 .

Fig. 68. The thin wedge A B, of which the height is one fifth of the length, being rolled round the cylinder $\mathrm{C}$, makes the screw $\mathrm{D}$, by means of which the weight $\mathrm{E}$ is capable of supporting a weight five times as great as F. P. 55 .

Fig. 69. A is a screw, and B the nut belonging to it. P.55.

Fig. 70. The endless screw A B acts on the teeth of the wheel C D. P. 55.

Fig. 71. The distance of the threads of the interior screw is four fifths of that of the exterior or perforated screw, and this distance is one thirtieth of the circumference. Hence the weight A is capable of sustaining a weight B 150 times as great as itself. P. 56.

Fig. 72. The apparatus for experiments on collision. Those balls which are not employed may be left behind the graduated arc, as at A and B; some of the strings have balls of half the weight of the rest, others have a small dish $\mathbf{C}$, on which balls of clay, or of wax softened with one fourth its weight of oil, may be supported. P. 58.

Fig. 73. If the ball $\mathrm{A}$ strike the ball $\mathrm{B}$ in the oblique direction $\mathrm{A} \mathrm{C}$, the ball $\mathrm{B}$ will be impelled in the direction C D perpendicular to the surface of contact; and the velocity $\mathrm{EC}$ being resolved into $\mathrm{EF}$ and $\mathrm{FC}$, the part $\mathrm{FC}$ will continue unaltered; and if the balls are equal, the part E F will be destroyed, so that the ball A will move after the stroke in the direction C G, excepting the effect of any accidental disturbance which may be derived from the resistance of the surrounding bodies. If we imagine a ball at $\mathbf{C}$ in contact with $\mathrm{B}$, in the direction $\mathrm{D} \mathrm{B}$, we may aim a blow at the centre of this ball, in order to drive the ball $\mathrm{B}$ to $\mathrm{D}$; and if $\mathrm{B}$ happen to be situated any where in the semicircle $\mathbf{D C G}$, the motion of $\mathbf{A}$ after the impulse will be in the direction B G or G B, if there be no resistance. When the ball $\mathrm{H}$ is reflected by a fixed obstacle, as by the cushion of a billiard table, at I, its velocity $\mathrm{K} \mathrm{I}$ may be resolved into the parts $\mathrm{K} \mathrm{L}, \mathrm{LI}$; the part $\mathrm{K} \mathrm{L}$ continues, and may be represented by $\mathrm{L} M$ equal to $K \mathrm{~L}$, the part $\mathrm{L} I$ is converted into $I \mathrm{~L}$ in a contrary direction, which when combined with $L M$ makes I M, the angle $L I M$ being equal to L I K. We may find the proper direction for striking any ball by reflection if we suppose a ball $\mathrm{N}$ in contact with the nearest point of the cushion, and making $\mathrm{N} \mathrm{O}$ equal to $M N$, aim at a ball supposed to be at $O$. In the same manner if we wish to impel the ball $P$ in the direction $P Q$ by a stroke of the ball $R$ after reflection at $S$, we first place a ball at $\mathbf{T}$ behind $\mathbf{P}$, and determine the direction $\mathrm{R} \mathrm{S}$ by aiming at a ball $\mathbf{U}$, as if we wished to strike a ball at $\mathbf{T}$ with a direct impulse. But in the case of a billiard ball, the rotation of the ball round its axis, which is not destroyed by the collision, will cause the ball to move, on account of the friction of the table, in a direction different from its first direction: thus the ball $\mathbf{C}$ will not go on to $\mathbf{G}$, but will strike the cushion between $\mathbf{C}$ and $\mathrm{D}$; and the ball $\mathbf{H}$, after reflection at $\mathbf{I}$, will proceed in a direction a little nearer to $\mathrm{N}$ than $\mathrm{IM}$; so that the imaginary ball $\mathrm{O}$ ought perhaps to be placed as far from the cushion itself as $M$, in order that the ball may be struck after reflection. P. 62 .

Fig. 74. Mr. Smeaton's apparatus for experiments on rotatory motion. P. 64 .

Fig. 75. The moveable centre of suspension being fixed at the distance of 5 inches from one of the balls, and 7 from the other, the vibration is performed at the same time as that of a pendulum 37 inches long. P. 65 .

Fig. 76. The three weights, supported on wheels, being drawn up the three inclined planes at the same time, by the action of three other equal weights, the middle weight arrives first at the top, the length of its plane being twice the height. P. 67 . 


\section{P L A T E VI.}

Fig. 77. The proportions of the diameters of the different parts of the double pullies being 3 to 2,5 to 2 , and 8 to 2 , the middle weight may be observed to rise the most rapidly. P. 67 .

Fig. 78. A wheel supposed to be capable of producing a perpetual motion; the descending balls, acting at a greater distance from the centre, but being fewer in number, than the ascending. In the model, the balls may be kept in their places by a plate of glass covering the wheel. P. 70.

Fig. 79. A, the inclination of cross lines generally most convenient for producing the effect of a tint, in drawing. B shows the effect of lines crossing each other perpendicularly, and $\mathbf{C}$ that of lines crossing too obliquely. Where the surface to be shaded is large, the separate lines or hatches should begin and end with a point, in order that the junction of the different portions may escape observation. P. 72 .

Fig. 80. Dr. Hooke's telegraph, in which the characters are arranged behind a screen, and drawn out as they are required. P. 77.

Fig. 81. Dr. Hooke's alphabet, with some other arbitrary characters for his telegraph. P. 77.

Fig. 82. A beam compass, with a scale. P. 78.

Fig. 83...85. Instruments for drawing arcs of large circles. P. 78.

Fig. 86. A pair of triangular compasses. P. 78.

Fig. 87. Marquois's scales, for drawing parallel lines. P. 79.

Fig. 88. A pen for ruling musical lines. P. 79.

Fig. 89. A pantograph. A being the centre of motion, $B$ the tracing point, and $C$ the describing point. $A B$ is always to $A C$ as $A D$ to $A E$, and the copy $F$ is similar to the original G. P. 79.

Fig. 90. A pair of proportional compasses. P. 79. 


\section{P L A T E VII.}

Fig. 91. A Sector. The scale of equal parts is marked L. As A B is to A C, so is $\mathrm{BD}$ to $\mathrm{CE}$; and if any line $\mathrm{BD}$ be placed with its extremities in the third division of the scale on each leg, the distance $\mathrm{C} \mathrm{E}$ between the seventh divisions will contain 7 equal parts, of which B D contains 3 ; and the same is true of any other numbers. P. 80 .

Fig. 92. A vernier, indicating $38 \frac{3}{10}$ of the divisions of its scale. P. 81 .

Fig. 93. A sliding rule. The slider being drawn out, so that the division marked 1 is opposite to 3 on the rule; all the other figures on the rule are triple of those which stand opposite to them. P. 82.

Fig. 94. A circular logarithmic instrument. The inner circle slides within the outer, and as it is represented in the figure, each number stands opposite to another which is twice as great. P. 82 .

Fig. 95. A steel chain, made by Ramsden. A, the screw for bringing the mark B precisely to the point required; C, a joint between the adjoining links; D, a cross joint at every tenth link; E, a pulley and weight for stretching the chain. P. 86.

Fig. 96. A micrometrical scale made by Troughton. The compound microscopes $\mathbf{A}$ and $\mathbf{B}$ are fixed nearly at the required distance on the scale $\mathbf{C} ; \mathbf{A}$ is then made to point exactly to a division of the standard scale $\mathbf{D}$ by means of the screw $\mathbf{E}$, and $\mathbf{B}$ to another division, at the required distance, by means of the screw $\mathrm{F}$, the fractional parts being added by the turns of the crew G. The scale $D$ is then removed, and the object to be compared with it is put in its place. P. 86 .

Fig. 97. A diagonal scale. The line A B contains 274 parts, of which the units of the scale contain 100 . P. 86 .

Fig. 98. The statuary's compass, seen sideways. The pin A B is forced down, till it is stopped by the moveable stud $\mathbf{C}$; the screw $\mathbf{D}$ fixes it in its angular position. It is also capable of motion round the axis $\mathrm{E} \mathrm{F}$, which is fixed by the screw G. P. 87 .

Fig. 99. An instrument for making drawings in perspective; the perforated sight may be drawn out to any required distance. The dotted lines show how a second frame may be applied instead of the sight, so as to answer the same purpose. P. 88.

Fig. 100. Illustration of the principles of perspective. A being the place of the eye, and B C the plane of projection, if $\mathrm{A} \mathrm{D}$ be parallel to E F, GH, and I K, $D$ will be their vanishing point, and $E D, G D$, and I D, their whole images : A L being parallel to $\mathrm{E} \mathrm{M}$ and I N, L will be their vanishing point, and $\mathrm{E} \mathrm{L}, \mathrm{I} \mathrm{L}$, their whole images : and A $\mathbf{O}$ being parallel to $\mathbf{P} \mathbf{Q}, \mathbf{O}$ will be its vanishing point. P. 89.

Fig. 101. A being the centre of the picture, A B the horizontal vanishing line, A C the vertical line, and D the point of distance, if a ground plan E F G H of any figure on the horizontal plane be placed in its true position with respect to $I K$, the bottom of the picture, the vanishing points of all its lines will be found by drawing $\mathrm{DL}, \mathrm{DM}, \mathrm{DN}$, and $\mathrm{D} \mathrm{O}$, parallel to those lines respectively; and the whole images of the lines will be $\mathrm{PL}, \mathrm{QM}, \mathrm{KN}$, and I $\mathrm{O}$, determining, by their intersections, the figure RST $\mathrm{T}$, which will be the projection of $\mathbf{E} \mathbf{F} \mathbf{H}$. The plan may also be drawn, in an inverted position, below the line $I K$, and the point of distance taken above $\mathbf{A}$ instead of below it. P. 89.

Fig. 102. A B being the whole image of the line represented by A C as a ground plan, and $\mathbf{D}$ the point of distance, we may find $\mathbf{E}$, the image of the point $\mathbf{C}$, by drawing $\mathbf{C D}$; or we may make $\mathbf{B F}=\mathbf{B D}$ and $\mathbf{A G}=\mathbf{A} \mathbf{C}, \mathbf{F} \mathbf{G}$ will then also cut $A$ B in the point E. P. 89 . 


\section{PLATE VIII.}

Fig. 103. The heights of the houses, windows, doors, and figures are determined by lines directed to the centre of the picture ; the true height being measured on the lines $\mathrm{AB}, \mathrm{CD}$, where the objects are supposed to touch the plane of projection. The distance EF, and all other parts of lines perpendicular to the picture, are measured by laying off the lengths of the originals, as $\mathrm{G} \mathrm{H}$, on the line $\mathrm{A} \mathrm{C}$, and drawing I E G, I F H, from I, the point of distance; which, in most cases, will be more remote from the centre of the picture than it is here made. The line $\mathrm{KL}$, and others parallel to A C, may be measured by the assistance of any point $M$ in the horizontal line, the distances, $\mathrm{N} \mathrm{O}, \mathrm{OP}$, being laid off on A C, or simply by reducing the scale in the proportion of MP to $\mathrm{ML}$. P. 89 .

Fig. 104. A circle thrown into perspective, by means of the circumscribed square, the points of contact being found by bisecting the sides. P. 89 .

Fig. 105. Two perspective delineations, and two orthographical projections of a cube, in different positions. For the orthographical projection, the ground plan being A B C D, the image of any point A, B, may be found by drawing A E, B F, perpendicular to the ground line; E G, F H, parallel to the line assumed for the direction of the centre of the picture, and $\mathrm{A} \mathrm{G}, \mathrm{B} \mathrm{H}$ parallel to the line of direction of the point of distance; the intersections $\mathrm{G}$ and $\mathrm{H}$ will then be the points corresponding to $A$ and $B$. P. 90.

Fig. 106. $\mathrm{A}$ is the orthographical projection of a sphere, with some of its circles; B the stereographical projection of the same circles. P. 90.

Fig. 107. A balance made by Fidler for the Royal Institution, nearly resembling those of Ramsden and Troughton. The middle column $\mathbf{A}$ is raised at pleasure by the cock B, and carries the round ends of the axis in the forks at its upper part, in order to remove the pressure on the sharp edges of the axis within the forks. The scales are occasionally supported by the pillars $\mathbf{C}$ and $\mathbf{D}$, which are elevated or depressed by turning the handle $\mathrm{E}$. The screw $\mathrm{F}$ serves for raising or lowering a weight within the conical beam, by means of which the place of the centre of gravity is regulated. The extent of the vibrations is measured on the graduated arc G. P. 97.

Fig. 108. A balance for the illustration of different kinds of equilibrium. When the scales are hung on the middle pins, A, B, which are in the same horizontal line with the support of the beam, the equilibrium is neutral, the weights acting as if the centre of gravity coincided with the point of suspension. If the scales be hung on the lowest pins $C, D$, the centre of gravity will be nearly in the line $C D$, and its path the curve E, which has its concavity upwards; but if the scales are hung on the pins $F, G$, the path of the centre of gravity will be convex upwards, and the beam will overset. In reality the true paths of the centre of gravity would be nearly in the curves $\mathrm{H}$ and $\mathrm{I}$, situated between the weights in the scales : but these are similar to the other curves. P. 97.

Fig. 109. When the equilibrium of a balance is tottering, the lower weight acts with the greatest advantage : thus the effect of the weight $\mathrm{A}$ is reduced in the proportion of $\mathrm{BC}$ to $\mathrm{DC}$, by the obliquity of the $\operatorname{arm~} \mathrm{CA}$, while the weight $\mathrm{E}$ acts on the whole length of its arm C F. P. 97.

Fig. 110. If A B C be a semicircle, and B D represent a given weight, and A D its counterpoise in one of the scales of an unequal balance, $\mathrm{D} \mathrm{C}$ will be the counterpoise in the other scale. It is obvious that $\mathbf{A C}$ is more than twice as great as BD. P. 97. 


\section{P LA T E IX.}

Fig. 111. A weighing machine. The platform supporting the weight rests on the pins A, B, C, D, at equal distances from the fulcra E, F, G, H; so that wherever the weight may be placed, it presses equally on the lever I K, at $\mathbf{L}$, and is counterpoised by a much smaller weight placed in the scale M. P.97.

Fig. 112. A steelyard resembling that of Mr. Paul, in which different weights may be employed. A, a loop to check the vibrations; $\mathrm{B}$, a scale to be suspended by the hook C. If great delicacy be required in the weights, the fractional parts may be expressed by the turns of a micrometer screw D, furnished with an index. P. 97.

Fig. 113. A bent lever balance. P. 98 .

Fig. 114. A spring steelyard : half the case being removed, to show the spring. P. 98.

Fig. 115. A B, the path of the centre of gravity of the human body, such as it would be described in walking, if the legs were inflexible. C D, the path described in running, on the same supposition. P. 100.

Fig. 116. The actual path of the centre of gravity, as it is usually described. P. 100 .

Fig. 117. An elastic column, compressed by a weight acting at the distance of one third of its depth from the concave surface; the compression being every where as the distance of the lines A B, A C. P. 107.

Fig. 118. An elastic column, extended by a weight acting at the distance of one third of its depth from the convex surface, the extension being every where as the distance of A B, A C. P. 107.

Fig. 119. An elastic column, compressed by a weight acting immediately on the concave surface : the compression extends only to the line A B, the parts beyond this line being extended. P. 107.

Fig. 120. A column bent, by a weight acting longitudinally, into the form of a harmonic curve: the line A BCD is the limit between the parts which are compressed, and those which are extended. P. 107.

Fig. 121. An elastic plate or rod, considerably bent by a weight acting at its extremity. P. 107.

Fig. 122. An elastic rod fixed at one end, and bent by its own weight. P. 108.

Fig. 123. An elastic rod supported at each end, and bent by its own weight. P. 108 . 


\section{PLATE X.}

Fig. 124. The manner in which a prismatic column is crushed by pressure, supposing the lateral adhesion to be simply proportional to the surface concerned. P. 112.

Fig. 125. The manner in which a column is crushed, supposing the lateral adhesion to be increased by pressure. P. 112 .

Fig. 126. The circle is as strong as the circumscribing square, supposing the adhesion proportional to the surface, the relative force of all its chords being equal. P. 113.

Fig. 127. The three circles are as strong as the circnmscribing parallelogram. P.113.

Fig. 128. A, the strongest form for a beam, cut out of a plauk of uniform depth, for resisting a longitudinal force; $\mathrm{B}$, the form into which it is bent; both curves being circular. P. 116.

Fig. 129. A, the strongest form for a beam, cut out of a plank of equable breadth, for resisting a longitudinal force which bends it into the cycloidal curve seen at B. P. 116.

Fig. 130. A, the strongest form for a square or turned beam or column, slightly bent by a longitudinal force : $B$, the form into which it is bent by such a force. P. 116 .

Fig. 131. The strongest form of a beam cut out of a horizontal plank, fixed at one end, and supporting a weight at the other. P. 116.

Fig. 132. The strongest form of a beam cut out of a vertical plank, fixed at one end, and supporting a weight at the other; the outline being parabolic. In practice the best method in such a case would be simply to reduce the depth at the end to one half of the whole, keeping the outline straight; in this manner one fourth of the timber would be saved. P. 116.

Fig. 133. The strongest form of a square or turned beam, fixed at one end, and supporting a weight at the other; the outline being a cubic parabola. P. 116 .

Fig. 134. The strongest form for the outline of a compound spring, supporting a weight at the end. P. 116.

Fig. 135. The strongest form for a beam cut out of a horizontal plank, fixed at one end, and supporting a weight equally distributed throughout its length; the outline being a parabola. P. 116.

Fig. 136. The strongest form for a beam cut out of a vertical plank, fixed at one end, and supporting a weight equally distributed throughout its length. P. 116.

Fig. 137. The strongest form for a square or turned beam, fixed at one end, and supporting a weight equally distributed throughout its length; the outline being a semicubic parabola, in which the cube of the thickness is as the square of the distance from the end. P. 116.

Fig. 138. The strongest form for a beam cut out of a vertical plank, for supporting its own weight; the outline being a parabola. P. 116.

Fig. 139. The strongest form for a turned beam, for supporting its own weight ; the outline being parabolic. P. 116.

Fig. 140. The strongest form of a beam calculated to resist the pressure of its own weight by lateral adhesion only. The outline is a logarithmic curve, which never comes into contact with the axis, and in order that the condition of equal strength may be possible, the beam must be loaded with a weight at its extremity, equal to that of the portion which is wanting to complete the figure. P. 116.

Fig. 141. The strongest form for a beam cut out of a horizontal plank, supported at both ends, and bearing a weight at the middle. P. 116.

Fig. 142. The strongest form for a beam cut out of a horizontal plank, supported at both ends, and bearing a weight equally distributed throughout its length ; the outline being parabolic. P. 116 .

Fig. 143. The strongest form for a beam cut out of a vertical plank, supported at both ends, and bearing a weight equally distributed throughout, the outline being elliptic. P. 116.

Fig. 144. The strongest form for a beam cut out of a horizontal plank, firmly fixed at both ends, and supporting a weight at the middle. P. 116.

Fig. 145. The strongest form for a beam cut out of a vertical plank, firmly fixed at both ends, and supporting a weight at the middle, the curves being parabolic. P. 116.

Fig. 146. The strongest form for a beam cut out of a vertical plank, and sup- 
porting every where a weight proportional to the distance from the extremity; the outline being a cubic parabola. P. 116.

Fig. 147. The strongest form for a square or turned beam, supporting every where a weight proportional to the distance from the extremity, and represented by the section of the same figure, which is a pyramid or a.cone. P. 116.

\section{P LA T E XI.}

Fig. 148. A machine for examining the strength of materials. The force is applied by means of the winch $\mathbf{A}$, which winds up the rope $\mathbf{B} \mathbf{C}$, passing over the first pulley, and under the second, which is directly under the point $D$, at which the force acts on the piece E F to be broken; the pullies slide on two parallel bars, fixed in a frame, which is held down by a point projecting at $\mathbf{G}$, from the lever $\mathbf{G} \mathbf{H}$, which is graduated like a steelyard, and measures the force. The piece to be broken is held by a double vice, $\mathrm{I}, \mathrm{K}$, with four screws, two of them hiding the other two in the figure : if a wire is to be torn, it may be fixed to the cross bar $\mathbf{L} \mathbf{M}$; and a substance to be crushed must be placed under the lever $\mathrm{NO}$, the end $\mathrm{N}$ receiving the rope, and the end $O$ being held down by the click, which acts on the double ratchet $\mathbf{O} \mathbf{P}$. The lever is double from $\mathbf{O}$ to $\mathbf{Q}$, and acts on the substance by a loop, fixed to it by a pin. P. 116 .

Fig. 149. The outline of a column diminished one fifth of its diameter, in two different ways : the side $\mathbf{A}$ being an arc of an ellipsis, of which the semidiameter $\mathbf{A ~ B}$ is the lesser semiaxis, joined at $\mathbf{A}$ to a right line $\mathbf{A ~ C}$, of one third of the length of the column, the part A D being cylindrical; the side DE is a cubic parabola, and may be drawn mechanically by fixing a straight ruler E F, in such a position that $\mathrm{DF}$ may be twice the diminution at $\mathrm{E}$, and then bending it to $\mathrm{D}$ : the diminution being every where as the cube of the distance from $D$. These two methods are compared in a contracted scale at $\mathbf{G}$ : the outer line represents the first method, and the next line the second; the third, which is nearest to $G$, the conchoid of Nicomedes, recommended by Chambers, said to be found in the columns of the Pantheon; the curve beginning at the base. Palladio fixes the rule at $\mathbf{A}$, and bends it to $\mathrm{H}$, which makes the curvature abruptly greater at $\mathrm{H}$. P. 122.

Fig. 150. A section of Mr. Smeaton's light house at the Eddystone. P. 122.

Fig. 151. Mr. Smeaton's mode of uniting tiers of stones by wooden pins and wedges. P. 123.

Fig. 152. A string of beads, suspended in equilibrium from two points, and remaining in equilibrium in an inverted position. The ends are supported by two pieces, which slide backwards and forwards, and are fixed by screws : the string is also tightened by turning a pin. P. 154.

Fig. 153. A system of bars, hanging in equilibrium, and supporting each other in the same form when inverted. P. 154 .

Fig. 154. A, a chain loaded, at equal distances, with other chains of such a length, as to represent the depth of the materials pressing on an arch of the form shown by the first chain, and holding it in equilibrium. B, an arch of a similar form. P. 154.

Fig. 155. A comparison of the curves which have various advantages for the construction of an arch supporting a horizontal road. The full line is an elliptic arc, somewhat less than half the ellipsis. The outside curve, which is also continued furthest down, is that which is calculated for resisting the pressure of materials acting like a fluid, or in the manner of wedges; the second dotted curve, for supporting the pressure of the materials above each part, supposed to act in a vertical direction only ; the third is a circular arc, making one third of a whole circle; the fourth is part of a logarithmic curve, which is nearly of equal strength with respect to the tendency of the materials to give way for want of lateral adhesion; and the fifth is composed of parabolic curves, showing the outline which would be strongest for supporting any additional weight placed on the middle of the arch. If the height were greater in proportion to the span, as usually happens in practice, there would be less difference between the curves. The radius of curvature at the summit being $\mathrm{A} \mathrm{B}$, the horizontal thrust is equal to the weight of the portion A B C D of the materials. P. 125. 


\section{P L A T E XII.}

Fig. 156. The middle arch of Black Friars Bridge. P. 126.

Fig. 157. A spherical dome, of which the lower parts are made thicker, in order that they may be of equal stability throughout. From $A$ to $B$ the dome is of equable thickness : below $\mathbf{C}$ and $\mathrm{D}$ the thickness cannot be increased sufficiently to procure an equilibrium, without the application of a chain or hoop, of which the section is represented at C, D. If the thickness were not at all increased, a hoop would be required at $\mathrm{E}, \mathrm{F}$, or still higher. P. 127.

Fig. 158. A section of the roof of St. Paul's Cathedral. The section of the dome consists of two circular arcs, of which the centres are a little beyond the axis: it is supported by carpentry, resting on a cone of brickwork. The internal dome is of brickwork only, and is open at the summit. P. 127.

Fig. 159. A section of the dome of the Pantheon at Rome. P. 127.

Fig. 160. A Tuscan column, with its pedestal, capital, and entablature. P. 127.

Fig. 161. A Doric column. P. 127.

Fig. 162. An Ionic column. P. 127.

Fig. 163. A Corinthian column. P. 127.

Fig. 164. A Composite column. P. 127.

Fig. 165. An elevation of the end of King's College Chapel, Cambridge ; show ing on one side the buttresses, the tower being supposed to be removed, and on the other the tower, which not only supplies the place of a buttress at the end, but assists also in supporting a considerable portion of the thrust in the direction of the length of the chapel; the roof, which is of stone, being vaulted in this direction as well as transversely. There is also a roof of carpentry, covered with lead above the stone roof. P. 128. 


\section{P L A T E XIII.}

Fig. 166. Joints for a tie beam. The joints at A and B cannot be more than half as strong as the entire beam, supposing the adhesion, produced by the pressure of the bolts, as strong as could be required. The joint at $\mathbf{C}$ is called a dovetail joint; its strength is a little less than that of $\mathbf{A}$ and $\mathbf{B}$, but the adhesion is more easily secured, since a force tending to separate the beams must tighten the joint. P. 128.

Fig. 167. Joints for a tie beam. The joint $A$, if sufficiently tight, may possess of the strength of the beam. The joint B may be as strong as the beam, if the adhesion were great enough, but it would be difficult to apply sufficient pressure to create such an adhesion, and if the beam were subject to be much shaken, the joint would be a very bad one. P. 128 .

Fig. 168. A good joint for a tie beam; the adhesion being secured by a slight diminution of the strength. P. 128.

Fig. 169. A, a simple 'scarfed joint, which may be tightened by a wedge at the centre ; it is not strong. B, a scarfed joint, which is much stronger. P. 129.

Fig. 170. A joint for a beam supporting a weight by its transverse strength. The junction might be made, if it were necessary, by means of a third piece, of which the limits are marked by the dotted line. The strength is but little diminished by the joint. P. 129.

Fig. 171. A beam supporting a weight by its transverse strength, joined to another by means of a third piece of half the depth, spliced or fished on below the beam, and secured by pins, and by blocks or joggles. The strength is a little greater than that of the original beam. The dotted lines show the proportion in which the strata are extended or compressed, the lower part of the original beam remaining in its natural state, without sustaining any pressure, as far as one fourth of the depth, and a little further. P. 129.

Fig. 172. A joint for a beám pressing obliquely against another. The dotted lines show the form of the tenon, which may occupy a considerable part of the breadth of the beam. The upper strap, $A$, is in the most usual situation, but the lower one, B, appears to afford greater strength, as it presses the beams more closely together, yet without any danger of crippling them; besides the advantage of having a firmer hold of the lower beam. P. 130.

Fig. 173. A joint for a horizontal beam suspended from a vertical one : the end of the tenon being dilated by wedges, and the whole secured by a strong strap. The tenon ought not to be wide, since it diminishes the strength of the horizontal beam. P. 130 .

Fig. 174. The straps, bent so as to deviate from the right lines joining their extremities in the degree that is here represented, have their strength reduced to about one seventh of that which they would have if straight. Thus, A B is only one seventh as strong as CD, supposing the substance inflexible. P. 130.

Fig. 175. The simplest form of a roof. A B, A C, are the rafters, and B C the tie beam; the weight of each half being represented by $\mathbf{A B}$, or $\mathbf{A} \mathbf{C}$, the thrust in the direction of the rafters will be $\mathbf{A} \mathbf{D}$, and the horizontal thrust each way $\mathbf{B} \mathrm{D}$ or C D. It is obvious that A D will be least when $B$ A C is a right angle. P. 130 .

Fig. 176. A common roof, with braces. A B is the king post, and B C, B D the braces. P. 130 .

Fig. 177. A kirb or mansard roof, the rafters of which hold each other in equilibrium. A B and C D are queen posts helping to support the tie beam. The piece A C acts as a strut, in supporting the pressure occasioned by the weight of the tie beam. The heads of the queen posts are not much thickened, in order to avoid the change arising from the unequal contraction of the wood. P. 130. 


\section{P L A T E XIV.}

Fig. 178. Three sketches for wooden bridges; the last requires no abutments. P. 131 .

Fig. 179. The centering used for building one of the arches of Black Friars Bridge. It was struck, or removed, by forcing back the compound wedges A, B, by the impulse of a battering ram. P. 131 .

Fig. 180. Modes of supporting a series of rods, for communicating alternate motion. $A$ is the best and most common method, the rods being suspended from a centre above them: at $B$ the centre of motion is below the rods. Where there is a declivity, the arrangement at $\mathbf{C}$ may be useful. The mode shown at $\mathbf{D}$ is also recommended in some cases. P. 133.

Fig. 181. A lever strengthened by a projecting frame. P. 133.

Fig. 182. A bent lever strengthened by a cross bar. P. 133.

Fig. 183. Hooke's universal joint. P. 133.

Fig. 184. A wheel with a crank, for producing alternate motion in a rod. P. 134, 257.

Fig. 185. A wheel with an inclined and undulated surface, for producing alternate motion in a rod, with the interposition of a friction wheel. P. 134, 257.

Fig. 186. A frame for guiding the motion of a point $\mathbf{A}$ in a direction nearly rectilinear, A B being to CD as C D to B E. [It is termed a parallel motion, Watt obtained a patent for it on the 28th of April, 1784.] The dotted line shows the path of the point A. P. 134, 257.

Fig. 187. A frame for producing a motion nearly rectilinear in the point $\mathrm{A}$. It may be applied to a pump rod B C, worked by a crank, or otherwise. P. 134, 257.

Fig. 188. A compound frame, for keeping two rods A B, C D, in a direction very nearly parallel. E F is 36 parts of the scale, F G, 64, G H and $\mathrm{H} \mathrm{I}$ each 80 ,

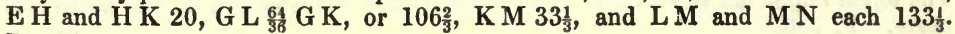
P. 134 . 


\section{PLATE XV.}

Fig. 189. The form of a wheel or pulley, on which a broad strap runs, the surface being convex : the wheel which drives it is of a similar form, but its upper part only is shown in the figure. P. 135.

Fig. 190. The teeth of two wheels, formed into epicycloidal curves, acting on planes: the dotted lines show the effective magnitude of the wheels. P. 135.

Fig. 191. The teeth of two wheels, formed into involutes of circles, described by uncoiling a thread from the dotted circles; the point of contact of the teeth being always in the straight line which touches both circles. P. 135 .

Fig. 192. Two surfaces formed into involutes of circles, revolving in contact with each other, the equidistant lines, drawn on them, continuing to meet each other throughout the revolution. P. 135.

Fig. 193. The pinion A is of the kind called a spur wheel; B is a crown wheel, or a contrate wheel. P. 136.

Fig. 194. The wheel and pinion are both bevilled; the faces of the teeth being directed to the point A. P. 136.

Fig. 195. Two wheels a little eccentric, acting on each other. P. 137.

Fig. 196. An eccentric contrate wheel, acting on a long pinion. P. 137.

Fig. 197. A machine for cutting the teeth of wheels. $A$ is the wheel, of which the teeth are formed by the revolving saw B, turned by the wheel and pinion C, D, by means of the handle $\mathrm{E}$, while the frame which holds the saw, moving on hinges, and resting on a spring, is depressed by the handle F, its place having been previously adjusted by the screw $\mathrm{G}$. The large plate $\mathrm{H} I$ contains a number of concentric circles, variously divided by points, into which the end of the spring I sinks at each step, 80 as to fix the apparatus in the required position. P. 137.

Fig. 198. A chronometer for measuring minute portions of time. The axis A B being turned, either by the handle $\mathbf{A}$ or by the weight $\mathbf{C}$, the balls $\mathrm{D}, \mathrm{E}$ fly out, and carry the weights F, G further from the axis ; in consequence of which the increased effect of friction retards the motion, when it becomes too rapid. The barrel $\mathrm{H}$ is turued in the mean time, with the axis, and is allowed to descend as the thread at $I$ is uncoiled, so that the point $\mathrm{K}$, which is pressed against it by a spring, describes on it a spiral, which is interrupted whenever the pin $K$ is touched. P. 147 .

Fig. 199. The fusee of a watch or clock, the general outline of which forms part of the hyperbola A B, in which the distance of each point from the axis C D is inversely as its distance from the line DE. P. 148. 


\section{P L A T E XVI.}

Fig. 200. A fusee with an auxiliary spring, for continuing the motion when the watch is wound up. The action of the main spring turns the fusee in the direction A B; the fusee acts on the ratchet wheel A B C by means of the click B, and this wheel impels the toothed wheel D E by the spring C B A, which is supposed to be seen through it. When the watch is wound up, this spring forces back the wheel A B C against the click $\mathbf{F}$, which serves as a fixed point, while the other end continues to act on D E, and to maintain the motion. P. 148 .

Fig. 201. The scape wheel A B, moving in the direction A C B, impels the pallets $\mathrm{D}, \mathrm{E}$ of the crutch or anchor, alternately in contrary directions. P. 149.

Fig. 202. A is the scape wheel, B and C the pallets of the common watch scapement. P. 149.

Fig. 203. The dead beat scapement. The teeth are first received on the flat or rather cylindrical surfaces $\mathbf{A}, \mathrm{B}$, on which they rest until the pendulum arrives near the middle of its vibration, when the teeth begin to act on the inclined surfaces terminating the pallets. P. 150.

Fig. 204. The horizontal scapement, for a watch. The tooth A rests first on the external surface of the cylinder, B C, and then impels it by its inclined face, in the direction B C; it afterwards falls on the concave surface D E, and lastly impels the cylinder in the contrary direction. P. 150.

Fig. 205. The duplex scapement. A B is the pallet, through which the cylinder, and the tooth which rests on it, are supposed to be seen, the point of the tooth being about to escape from the notch towards C. The short tooth D next impels the point of the pallet, and the long tooth $\mathbf{E}$ falls on the cylinder. It first rests on the convex surface, and then drops into the notch, which causes a slight recoil in the wheel, and passes by, the tooth $\mathrm{F}$ being beyond the reach of the pallet; but on its return, the tooth falls again into the notch; and when it escapes, the pallet is impelled as before. P. 150 .

Fig. 206. Mr. Mudge's watch scapement. A, the scape wheel, and one of the subsidiary springs, seen from above; B, a general view of the balance, with both the subsidiary springs, seen from one side. The point of one of the teeth rests at $\mathbf{C}$ on the end of the pallet, which is bent so as to detain it until the pin $D$, which is attached to the balance, sets it a liberty, by striking against the arm E; this arm is then carried on by the balance, to the end of its vibration, and impels it in its return, until the pallet meets the next tooth. The other spring acts alternately in the same manner, but in a contrary direction. P. 151 .

Fig. 207. An improvement on Mr. Cumming's scapement for a clock. The tooth $\mathbf{A}$ is seen resting on a flat surface at the end of the pallet $B$ : it is disengaged by the descent of the opposite pallet into the position in which it is represented, the pallet $\mathrm{B}$ being impelled by it at $\mathrm{C}$. This pallet continues resting on the flat end of the tooth, until the pin D of the pendulum strikes against the arm E, which is carried before it, and impels the pendulum in its descent, until the pallet B acquires the situation in which the opposite pallet is represented, and sets that pallet at liberty from the tooth $\mathrm{E}$, which has raised it. The situation and magnitude of the weights G, H, may be adjusted at pleasure. P. 151 .

Fig. 208. Mr. Arnold's watch scapement. The pin A, projecting from the verge or axis of the balance, moving towards $\mathrm{B}$, carries before it the spring $\mathrm{B}$, and with it the stiffer spring $\mathrm{C}$, so as to set at liberty the tooth $\mathrm{D}$, which rests on a pallet projecting from the spring. The angle $\mathbf{E}$ of the principal pallet has then just passed the tooth $\mathbf{F}$, and is impelled by it until the tooth $\mathbf{G}$ arrives at the detent. In the return of the balance, the pin A passes easily by the detent, by forcing back the spring $\mathbf{B}$. The screw $\mathrm{H}$ serves to adjust the position of the detent, which presses against it. P. 151.

Fig. 209. Mr. Earnshaw's scapement. A is the unlocking pallet, B the spring on which it acts, $\mathrm{C}$ the detent, holding the tooth $\mathrm{D}$ by a pin ; $\mathrm{E}$ is the point of the principal pallet first impelled by the tooth F, G is the tooth next locked, and $H$ the adjusting screw. P. 151.

Fig. 210. A gridiron pendulum, consisting of three bars of iron, and two of a mixture of zinc and silver. P. 154.

Fig. 211. A compensation balance, as employed by Arnold. The outside of the 
hoops A, B is of brass, the inside of steel : the weights C, D are screwed backwards and forwards, in order to obtain the requisite degree of compensation. The weights $\mathrm{E}, \mathrm{F}$ are employed to regulate the mean rate of the watch, and $\mathrm{G}, \mathrm{H}$, and $\mathbf{I}$, for adjusting it to all positions with respect to the horizon. P. 154 .

Fig. 212. The compound plate A B rests on two supports, which are adjusted to a proper distance by turning the double screw $\mathrm{C}$, the flexure of the plate by heat raising the bar $D$, which supports the pendulum, while its effective length is determined by a fixed clip, which is seen below the plate. P. 154.

\section{P L A T E XVII.}

Fig. 213. A jack for raising weights by the alternate motions of a lever, the clicks on each side being detained in the teeth of the ratchets by the assistance of the springs in which they terminate, and which are connected together. P. 157.

Fig. 214. The mode of supporting a tackle for raising stones in building; the summit of the triangle, which is composed of three poles, being raised or lowered by means of a rope and pullies. P. 159.

Fig. 215. A method of raising weights obliquely, by means of a rope passing over a pulley, which is drawn along horizontally. P. 159 .

Fig. 216. A B, a section of an inclined plane, belonging to the Duke of Bridgwater's canal; the boats are drawn into the locks at $\mathbf{A}$, which are then filled with water; $\mathrm{C}$ is the plan of the windlass, by which the descending and ascending boats are connected together, and which is turned by a winch; D and E are the locks. P. 159.

Fig. 217. A crane, with an oblique walking wheel, for oxen or horses. The wheel is taken from a mill of Leupold. P. 161 .

Fig. 218. A crane with a wheel and break like Mr. White's. The man walks at any required distance from the axis of motion, and pushes forwards the lever $A$, which moves the bar B C, connected to the same axis, and removes the break C D from the circumference of the wheel. P. 161.

Fig. 219. A lewis, for raising stones. P. 161.

Fig. 220. When the centre of gravity $A$ is twice as far from one of the porters $B$, as from the other $\mathrm{C}$, the first bears one third of the weight, the other two thirds. P. 162 .

Fig. 221. When the centre of gravity $\mathbf{A}$ is above the line joining the points of support B, C, the load is divided in the ratio of the segments C D, BD, terminated by the vertical line A D ; but it may be supported by two equal forces in the directions $\mathrm{BE}, \mathrm{CF}$, found by making $\mathrm{GH}$ equal to $\mathrm{BG}$, and joining $\mathrm{CH}$; the angle G B E being equal to $\mathrm{GHF}$; the forces and the weight may then be represented by the lines $\mathrm{C} I, \mathrm{I} K$, and $\mathrm{C} \mathrm{K}$. P. 162.

Fig. 222. A roller with two wheels fixed on its ends, by means of which the slab resting on it may be moved to a considerable distance without leaving the roller behind. P. 164 .

Fig. 223. Mr. Garnet's rollers for diminishing friction : their axes being loosely connected by a ring, in order to keep them in their places. P. 164 . 


\section{P LATE XVIII.}

Fig. 224. A pair of friction wheels, supporting one end of the axis of a wheel. P. 164 .

Fig. 225. The centre of the wheel A B, passing over the obstacle $\mathbf{C}$, describes the path DE; that of the larger wheel F G, the path H I, which is less steep. P. 164.

Fig. 226. The centre of the wheel A B describes the curved path C D, in passing over the obstacle $\mathrm{E}$, while that of the larger wheel $\mathrm{F} \mathrm{G}$ has an angle at $\mathrm{H}$. P. 164 .

Fig. 227. The wheel A B, moving on a soft road towards B, has to overcome the resistance of the earth at C. P. 165 .

Fig. 228. A section of the wheel of a carriage, a little dished, or inclined outwards. P. 166.

Fig. 229. A B and CD being the straps or braces by which a coach is suspended, if the centre of gravity be at E, F, or G, it must move, when the carriage swings, in the curve passing through the respective point. P.167.

Fig. 230. The mode of harnessing two horses, so as to make them draw conveniently together: when either horse advances so far that the bar A B assumes the position $\mathrm{CD}$, the foremost horse has the disadvantage of acting on a lever equivalent only to E F, while the other horse acts on E C. P. 167 .

Fig. 231. A sugar mill. The axis $A$ is turned either by animal force or by water : the liquor is collected in the trough $\mathbf{B}$, and runs off in the channel $\mathbf{C}$. The openings $\mathrm{D}$ are for the purpose of adjusting the axes of the rollers. The canes are supplied by the hands of the workmen. P. 170 .

Fig. 232. A glazier's vice. The vacuity in the middle shows the form of the section of the lead which is drawn through it. P. 171.

Fig. 233. A forge hammer, elevated by the plugs, projecting from an axis, either at $\mathbf{A}$, or more conveniently, at $\mathbf{B}$, and thrown forcibly against the wooden spring C. P. 171 .

Fig. 234. An engine for driving piles, on Vauloué's construction. The horses, drawing at $\mathrm{A}, \mathrm{B}$, raise the weight $\mathbf{C}$, held by the tongs $\mathrm{D}$, fixed in the follower $\mathbf{E}$, which are opened, when they reach the summit, by being pressed between the inclined planes $F, G$, so as to let the weight fall. At the same time the lever $\mathbf{H}$ is raised by the rope I, and presses on the pin $K \mathrm{~L}$, so as to depress the lever $M N$, and draw the pin $O$ out of the drum $\mathbf{P} Q$; the follower then descends, and uncoils the rope, its too rapid motion being prevented by the counterpoise $R$, acting on the spiral barrel $Q$. The motion is regulated by the fly $S$, the pinion of which is turned by the great wheel T. P. 173.

Fig. 235. The rollers of the slitting mill. P. 174 .

Fig. 236. A simple plough. A is the coulter, for dividing the ground; B the share, fixed on the mould board $\mathbf{C}$, for turning it to the right hand; D is the rest, and E, F, the handles. P. 175.

Fig. 237. Section of a threshing mill. The corn is drawn in by the rollers or feeders $\mathrm{A}, \mathrm{B}$ : it is beaten by the revolving beaters $\mathrm{C}, \mathrm{D}$, and the straw is drawn out by the rakes $\mathrm{E}, \mathrm{F}$, which discharge it at $\mathrm{G}$; the grain falling through the arched bottoms H I, I G, which are formed like sieves. P. 178 .

Fig. 238. A corn mill, with some of the improvements made in America, by $\mathrm{Mr}$. Ellicott and Mr. Evans. The corn being poured into the funnel A, is conveyed by the revolutions of a spiral $\mathrm{BC}$, to $\mathrm{C}$, whence it is raised, by the chain of buckets $\mathrm{C} D$, to be cleaned by the revolving sieve $\mathrm{E}$, and the fan $\mathrm{F}$; it is then deposited in the granary $\mathbf{G}$, which supplies the funnel or mill hopper $\mathbf{H}$; this being perpetually agitated by the iron axis of the upper mill stone, shakes it by degrees into the perforation of the stone; it escapes, when ground, at I, and is conveyed, by means of the carrier $\mathrm{KL}$, and the elevator $\mathrm{LM}$, to the cooler $\mathrm{N}$, where it is spread on a large surface : it passes afterwards to the bolter $O$, and is received in the binn $P$, from whence it is taken to be packed in sacks or barrels. Q represents the surface of a mill stone, cut into furrows, in order to make it act more readily on the corn. P. 179 . 


\section{PLA T E XIX.}

Fig. 239. The surfaces of the fluid in the bent tube A B remain on the same level, in the same manner as if the tube were absent, and the fluid made a part of that which is contained in the reservoir C D. P.197.

Fig. 240. The bucket A being suspended by the rope B, and made to revolve rapidly round its axis, the surface of the water assumes a parabolic form. P. 198.

Fig. 241. A heavier fluid being contained in the upper part of the bent tube A B, which is immersed in the lighter fluid, filling the vessel $C D$, the fluid in the tube remains in a state of tottering equilibrium, when its surfaces are in the same level. P. 198.

Fig. 242. The fluid A B C presses on the bottom of the vessel B C with the same force as if the vessel were of the form B CDE. P. 199.

Fig. 243. The portion A B C D of the fluid being supposed to be congealed, and then to form a part of the vessel, the pressure on the bottom would remain unaltered. P. 199.

Fig. 244. The weight A may be supported by the pressure of a small quantity of fluid, either by making the surface of the vessel B C very large, and the height of the tube D E moderate, or, while the vessel F remains of a moderate size, by making the height of the tube G H very great. P. 199.

Fig. 245. The pressure on any small part of the side of the vessel A B, at C or D may be represented by the line $\mathrm{CE}, \mathrm{DF}$, and the whole pressure on the side by the triangle B G, of which the centre of gravity is at $\mathrm{H}$; and if the side A I be supported by a single prop, it must be placed at the point $K$, the height of which is equal to that of H. P. 200.

Fig. 246. If the height of the surface $A$ above $B$ be to $B C$ as the specific gravity of the fluid in B C to that of the fluid in A B, the fluids will support each other. P. 201.

Fig. 247. Two square beams floating at the depths shown at $A$ and $B$, will have a certain degree of stability, but if they sink, as at C, they will overset. But a beam of the breadth shown at D will always float securely. P. 202.

Fig. 248. A jar containing images of fishes, with bubbles of air in them, which sink when the cover of the jar is pressed with the hand. P. 202.

Fig. 249. Dr. Hooke's semicylindrical counterpoise, by means of which a vessel is kept always full. P. 203.

Fig. 250. The form into which the flexible bottom of a cistern would be bent by the pressure of the water; the curve is the same as that into which an elastic rod would be bent by forces acting at A and B. P. 203 .

Fig. 251. The bottle A, containing air and mercury, has the tube A B fitted into it: and when the jar CD, in which it is enclosed, is exhausted by means of the air pump, the elasticity of the air in the bottle forces the mercury up the tube. P. 204.

Fig. 252. An instrument for showing the buoyant effect of the air, called by Boyle a statical baroscope ;* the index A shows, on the scale B C, the degree in which the ball $\mathrm{D}$ is obliged to descend, by the diminution of the weight of the air. P. 206.

Fig. 253. The line 0 denoting the natural density of the air, the line $1 \mathrm{~A}$ next above it shows the degree in which the air is expanded at the height of a mile, and I B the density of the air at the same height; in the same manner $10 \mathrm{C}$ shows the expansion of the air at the height of 10 miles, and $10 \mathrm{D}$ its density ; and $5 \mathrm{E}$, below the line, the density which it would acquire at the depth of 5 miles below the earth's surface. The lines A C, D B E, are of the kind called logarithmic curves. P. 206.

Fig. 254. The box or bason, in which the mercury of the common barometer is contained : $\mathbf{A}$ is a float for adjusting the beight, by means of the screw $\mathbf{B}$, operating on the leather which forms the bottom of the cavity. P. 209.

$$
\text { * Pl. Tr. 1665, p. } 231 .
$$




\section{P LAT E XX.}

Fig. 255. A jet or vein of a fluid, passing through an orifice in a thin plate in any direction, and contracted after its escape, in consequence of the lateral motions of the particles which flow towards the stream, nearly in the directions of the lines here drawn. P. 212.

Fig. 256. A stream flowing through a short cylindrical pipe, compared with another flowing through a diverging conical pipe, the directions of the motions of the particles appearing to be nearly similar in both cases. P. 213.

Fig. 257. In an experiment of $D$. Bernoulli, the water flowing through the conical pipe A drew up water through the tube $\mathrm{B}$ from the vessel $\mathrm{C}$; in another of Venturi, the water flowing through the cylindrical pipe $\mathbf{D}$ raised water through the tube E. P. 213.

Fig. 258. A siphon, through which a fluid runs from the higher vessel into the lower one. P. 215.

Fig. 259. A fluid flowing through a vertical pipe, and filling a vessel to a height nearly equal to the length of the pipes, while it is discharged through a similar horizontal pipe. P. 216 .

Fig. 260. Subterraneous cavities, with outlets in the form of siphons, through which they do not begin to discharge any water till they are nearly full; the lower one will then continue to run till it be empty. In the mean time either of them may keep up a constant stream by other passages. P. 217.

Fig. 261. A tube turned up and contracted, so as to throw out the fluid contained in it, in a jet, which rises very nearly to the height of the fluid in the tube. P. 217 .

Fig. 262. The forms of jets issuing from various parts of a reservoir, the amplitude A B being twice C D, and A E four times F G. P. 217.

Fig. 263. A series of waves, moving in the direction A B, and reflected by the obstacle $\mathrm{B}$, lose the appearance of progressive motion, and vibrate up and down within the limits of the curves A C D E B, and F G H I K; the elevation and depression become however twice as great as before reflection. P. 219.

Fig. 264. A series of waves diverging from a centre A, and striking a fixed obstacle B C, are reflected by it into the same form as if they proceeded from the centre $\mathbf{D}$, at an equal distance on the opposite side of the surface B C. P. 219.

Fig. 265. An apparatus for observing the motions of waves excited in a fluid poured into the trough $\mathrm{A} \mathrm{B}$, by the vibrations of the elastic wire $\mathrm{C}$, loaded with a moveable weight $\mathrm{D}$; the shadow of the waves being thrown on a screen $\mathrm{E}$ by the lamp F, through the bottom of the trough, which is of glass. P. 220.

Fig. 266. A series of waves, diverging from the centre A, and passing through the aperture B C, extend themselves on each side so as to fill the space B C D E, while they affect the parts without this space much less sensibly. P. 220, 360 .

Fig. 267. Two equal series of waves, diverging from the centres $\mathrm{A}$ and $\mathrm{B}$, and crossing each other in such a manner, that in the lines tending towards $\mathrm{C}, \mathrm{D}, \mathrm{E}$, and F, they counteract each other's effects, and the water remains nearly smooth, while in the intermediate spaces it is agitated. P. 220, 364 . 


\section{P LA T E XXI.}

Fig. 268. A stream of air being forced through the pipes A and B, the mercury in the barometer C D falls from C to D. P. 225 .

Fig. 269. A stream entering the reservoir A, by the pipe B, carries with it all the water C, which stands above the level of its upper surface. P. 226.

Fig. 270. The ball $A$ is permanently supported by the jet $B$, because when it falls into the position here represented, the centrifugal force of the water at $\mathbf{A}$ carries it back to the middle of the jet. P. 226.

Fig. 271. A plate, bent into the form A B C, turning on the centre B, is impelled by a stream of air D in the direction C D. P. 226.

Fig. 272. A cylinder moveable on an axis, with two curved pipes inserted in its lower part, seen from above. The stream A enters at the top of the cylinder, and is discharged by the orifices $\mathbf{B}, \mathbf{C}$, so as to turn the vessel in the direction $\mathbf{B} \mathbf{D}$. P. 229 .

Fig. 273. A jet of a fluid, striking on an obstacle of equal diameter, and separated by it so as to continue its motion obliquely. P. 229.

Fig. 274. The whole resistance directly opposed to the surface A B being represented by $\mathrm{B} \mathrm{C}$, the portion which, according to the principles of the resolution of forces, ought to act on the wedge A B D, is represented by B E; and in the same manner the resistance on A B F is to the whole as B G to B C. P. 230.

Fig. 275. The form of the dead water moving before an obtuse body is nearly like that of A B C; and the form adapted for moving through the water with the least possible resistance like A B D C. P. 231.

Fig. 276. The direction in which the particles of a fluid are supposed to move when they strike against a concave surface. P. 232.

Fig. 277. A hydrostatic balance. P. 235.

Fig. 278. Mr. Nicholson's hydrometer, to be employed with weights, for finding the specific gravity of fluids or solids. P. 236.

Fig. 279. A spirit level. P. 237.

Fig. 280. An overflowing lamp. The hemispherical counterpoise, which is so loaded, that its centre of gravity is at A, raises the surface of the heavy fluid B the higher as it is more exhausted, so that the oil $\mathbf{C}$ is always forced up nearly to the level of the wick at $D$. The oil is poured in by a pipe, in the middle of the cylindrical column. The air holes may be made wherever it is most convenient. P. 237.

Fig. 281. A section of an embankment, of a proper form to be opposed to the sea, with a drain passing through it, and a valve at its opening. P. 238 .

Fig. 282. The form recommended for the section of a river or canal. P. 238 .

Fig. 283. A B shows the strongest form for a vertical beam, fixed above and below, and calculated to resist the pressure of a fluid; the greatest thickness being at $\mathrm{C}$; and $\mathrm{DE}$ is the outline of a series of horizontal planks, of such a thickness as to afford equal strength throughout the sluice or floodgate. P. 239.

Fig. 284. A box, with a valve supported by a hollow ball, for letting out air from pipes, when it is below the level of the reservoir. P. 241.

Fig. 285. Two methods of letting out air from pipes, when it is above the level of the reservoir; A a valve with a stopcock near it ; $B$ a vessel of water, screwed on for receiving the air; to be replenished with water as it becomes empty. P. 242.

Fig. 286. A section of a compound stopcock, which receives a fluid from either of the pipes A, B, or C, into a cavity which descends a little in the direction of the axis, and communicates with the pipe $\mathrm{D}$, by means of one of the bores represented by dotted lines, according to the position into which the moveable cylinder is turned. P. 242 .

Fig. 287. Valves of different kinds; $\mathbf{A}$ the common clack valve; $\mathbf{B}$ a double clack valve, consisting of two semicircular valves; $C$ a pyramidical valve, consisting of four triangular pieces; D a circular valve turning on an axis; $E$ a steam valve of metal, sometimes called a $\mathrm{T}$ valve; F, a valve of oiled silk or bladder, supported by a grating, for air. P. 242. 


\section{PLATE XXII.}

Fig. 288. Mr. Woltmann's hydrometrical fly. The plates A, B, are so adjusted by experiment, as to move exactly or very nearly with the velocity of the wind, a few degrees being allowed as a compensation for the retardation of friction. The cord $\mathbf{C}$ is drawn up, and the wheel D is caused to revolve, at a time observed by a stop watch; and its surface is graduated so as to number the revolutions of the fly. P. 243.

Fig. 289. An apparatus for measuring a ship's way, resembling Captain Hamilton's. $\Lambda$ is a funnel partly covered, B a part of the ship's keel, $C$ the upper part of the pipe $\mathrm{D}$, in which the smaller pipe $\mathrm{EF}$ slides in a collar of leathers, so as to have the orifice F level with the surface of the water. This pipe has a small aperture at the bottom, which limits the magnitude of the stream discharged into the vessel G, the end $\mathrm{F}$ being considerably larger. The tube $\mathrm{H}$ serves as a gage, to measure the velocity at any given time. P. 243.

Fig. 290. An overshot wheel, on which the water is admitted in a retrograde direction, so as to run off in a continued stream; at the lower part of the wheel it is retained in the buckets partly by the assistance of a sweep. P. 245.

Fig. 291. A breast wheel, with a sweep. P. 246.

Fig. 292. An undershot wheel. P. 246.

Fig. 293. A the form of the sail of a windmill : B the best inclination for each part of the sail A, according to Smeaton's experiments. P. 247.

Fig. 294. A kite supported by the wind, of which the force acts nearly in the line A B, perpendicular to the surface of the kite; and this, compounded with the force of the cord A C, produces the result $\mathrm{AD}$, which sustains the weight of the kite. P. 247.

Fig. 295. A ship working against a wind; the force of the wind acting nearly in the direction A B, perpendicular to the sails, the ship's real course is B C, the angle C B D being the lee way. P. 249.

Fig. 296. The anoria, or noria, used in Spain, for drawing water, by a series of earthen pitchers, connected by ropes, and passing over a sprocket wheel. P. 250 .

Fig. 297. An undershot waterwheel, carrying fixed buckets, which raise a portion of water, and deliver it into a trough, furnished with a projection, which stands under the buckets, at the upper part of the wheel. P. 250 .

Fig. 298. A throwing wheel, for draining fens, worked by a windmill or otherwise, and carrying the water upon a sweep from a lower to a higher level. P. 250.

Fig. 299. The rope pump of Vera, for raising water by means of friction : the rope is kept stretched by a pulley under the water, which is loaded with a weight, and slides in a groove. P. 251 .

Fig. 300. The screw of Archimedes, nearly as described by Vitruvius. P. 251.

Fig. 301. The screw of Archimedes, as recommended by D. Bernoulli. P. 251.

Fig. 302. A waterscrew, revolving within a fixed cylinder. P. 252.

Fig. 303. The spiral pump of Wirtz. P. 253. 


\section{PLA T E XXIII.}

Fig. 304. A centrifugal pump. The machine is first filled through the funnel $A$, and when it is made to revolve, the water is discharged into a circular trough, of which a section is seen at $B$ and $C$. The valve at $D$ remains shut while the pump is filling. P. 253.

Fig. 305. A pump consisting of two plungers, continued nearly to the height at which the water is delivered. P. 254.

Fig. 306. Lahire's double forcing pump. When the piston is depressed, the water enters the barrel at the valve $A$, and goes out at $B$; when it is elevated, it enters at $\mathbf{C}$ and escapes at D. P. 254.

Fig. 307. The common piston, coated with leather. P. 254.

Fig. 308. Mr. Bramah's press. The pump A forces the water through the pipe $B$ into the barrel $\mathrm{C}$, in which it acts very powerfully on the large piston $\mathrm{D}$, and raises the bottom of the press E. P. 254 .

Fig. 309. The common sucking pump. P. 254.

Fig. 310. A bag pump, the bag or puff A being extended and contracted by the motion of the piston. P. 255.

Fig. 311. A lifting pump, the piston rod AB being drawn up by a frame. P. 255 .

Fig. 312. A sucking pump, converted, by the addition of a collar of leathers at A, into a forcing pump. P. 255.

Fig. 313. A fire engine, on a construction similar to some machines described by Ramelli. A B is the piston, working within a cylindrical barrel, and moved by the handles $\mathbf{C D}$. When the end $\mathbf{C}$ is depressed, the water enters through the valves $\mathbf{E}$ and $F$, and is discharged at $G$ and $H$; when $D$ is depressed, the water enters at $I$ and $\mathbf{K}$, and is discharged at $\mathbf{L}$ and $M$, into the air vessel $N$, whence it is expelled by the pipe $O$. The pipes $P$ and $Q$ may be united, if it be required. P. 255 .

Fig. 314. From Ramelli. The wheel A B, revolving in the direction B A, carries a portion of water $\mathbf{C}$ between itself and the sweep $\mathrm{DE}$, which is intercepted by the slider F, and forced up the pipe E G. P. 256.

Fig. 315. From Ramelli. The roller A, revolving within the reservoir B C, which is nearly cylindrical, carries with it the slider $\mathrm{D} \mathrm{E}$, which is made to sweep the internal surface of the cylinder from $\mathbf{C}$ to $\mathbf{F}$, by means of a projecting surface acting on the end $D$, so that the water $G$ is forced through the pipe F. P. 256.

Fig. 316. From the cabinet of Mr. Servière. The wheels A and B carry, during their revolution, a quantity of water from $C$ to $D$, or from $D$ to $C$, according to the direction in which they are turned. P. 256.

Fig. 317. Mr. Gwynn's patent water engine. The valve A is kept, partly by means of the spring $\mathbf{B}$, but still more by the pressure of the water, in contact with the roller or piston $\mathbf{C}$, which revolves within the box $\mathbf{D E}$, and sweeps it from $\mathbf{E}$ to $\mathbf{F}$, so that the portion of water $\mathrm{G}$ is forced, during each half of a revolution, into the pipe $\mathrm{F}$; or is drawn from $\mathrm{F}$ to $\mathrm{E}$, when the roller revolves in a contrary direction. P. 256 .

Fig. 318. A chain pump. P. 257.

Fig. 319. The mechanism of Höll's acting pump. In the position of the stopcock $A$ B, here represented, the water flows out of the barrel $C$, and the piston $D$ is allowed to descend. The rod $\mathbf{E}$ then turns the stopcock, and the barrel $\mathbf{C}$ communicates only with the pipe $\mathrm{F}$, whicb fills it, and forces up the piston, until the stopcock is turned back to its former position. P. 257.

Fig. 320. The hydraulic air vessels of Schemnitz. The reservoir A being filled with water, and $B$ with air, and water being poured into the funnel $C$, the air in $B$ acts by the pipe $\mathrm{D}$ on the water in $\mathrm{A}$, and forces it up the pipe E. P. 258.

Fig. 321. A being the high water mark, and $B$ the low water mark, the vessels $\mathbf{C}$ and $\mathrm{D}$ are filled at high water from below, the air being suffered to escape by a stopcock, which is opened by the fall of the ball F; at low water the air will enter the vessel $D$ at $B$; and before the next high water, the water $C$ will be forced up the pipe E. P. 258.

Fig. 322. The fountain of Hero. Its operation resembles that of the hydraulic air vessels, fig. 320 ; but the pipe $\mathrm{D}$ here ascends. P. 258.

Fig. 323. The hydraulic ram of Montgolfier. When the water in the pipe A B has acquired a sufficient velocity, it raises the valve $\mathbf{B}$, which stops its passage, so that a part of it is forced through the valve $C$, into the air vessel $D$, whence it rises through the pipe E. P. 259. 


\section{P L A T E XXIV.}

Fig. 324. The cupping instrument of Hero. The cavity A was partly exhausted by applying the mouth repeatedly to the pipe $B$, the stopcock $B$ being turned after each application. When the stopcock $C$ was opened, the air at D in contact with the skin was also rarefied, and the effect of suction was produced. P. 260, 276 .

Fig. 325. Mr. Cuthbertson's air pump. When the piston rod A is depressed, it leaves the piston $\mathrm{B}$ a little behind it, so as to make an opening between two conical parts which are ground together, and the air escapes from the lower part of the barrel into the upper part; when it is elevated, the whole piston is raised, and a wire, which slides through the axis of the rod, raises a small valve at the bottom of the barrel, which leads to the receiver C, by the tube D E : the air is forced from the upper part of the barrel through a valve in the oil vessel $F$, whence the oil runs back, when it overflows, by a tube leading to the mouth of the barrel; and if this tube be stopped by turning its cock, the air may be condensed into a receiver fixed at $\mathbf{G}$. At $\mathbf{H}$ is a long gage, with a barometer immersed in the same bason of mercury. The piston rod, which is hollow, has a perforation a little above A, to admit the oil, in order that the wire may work freely in it. P. 261.

Fig. 326. The two flies $A$ and $B$ being caused to revolve with equal velocities by the descent of the weight $C$, they continue to move for an equal length of time in the vacuum of the air pump. P. 261.

Fig. 327. The air in the bottle A expands, when the receiver B is exhausted, and causes the water to rise in a jet. P. 261.

Fig. 328. A pear gage; to be suspended in a receiver by a hook like that which is shown in fig. 325. P. 262.

Fig. 329. A condenser, with screws, for confining the receiver. A is a gage for showing the degree of condensation; $B$ the piston of the syringe, with a valve of the best kind, which is conical, and is confined by a spiral spring. But in common, the valves are made of leather, with a plate of metal to strengthen it. P. 262.

Fig. 330. A diving bell. A is the forcing pump, B a stopcock for letting out the heated air, $\mathrm{C}$ a strong glass for giving light, $\mathrm{D}$ a float for the security of the diver. P. 262.

Fig. 331. Laurie's hydraulic bellows. When the vessel $\mathbf{A}$ is raised, the air enters at the valve $B$; when it is depressed, the valve $B$ shuts, and the air is forced through the pipe $C D$, which conducts it to the reservoir $E$, where it is confined by the valve F, and forced by the pressure of the water through the pipe G. P. 263.

Fig. 332. Mr. Watt's gasometer. The pressure is regulated by the magnitude of the weights $A$ and $B$, which act by the spiral fusees C, D, so as to sustain a part of the weight of the inverted vessel, represented by the exterior dotted line. The gas is admitted at $\mathbf{E}$ or $\mathbf{F}$, and is delivered at $\mathbf{G}$. $\mathbf{G ~ H}$ is a gage for showing the height of the water within and without the moveable vessel. I is a cock for letting off the water. P. 263.

Fig. 333. The shower bellows. The stream A, passing through the strainer B, carries with it a quantity of air through the pipe $C$, which rises to the upper part of the air vessel D, and is discharged by the pipe E. P. 263.

Fig. 334. The centrifugal bellows. By the revolution of the fly, the air is caused to enter at A, and is discharged at B. P. 264.

Fig. 335. The original steam engine of Savery. The vessel A being filled with steam from the boiler $B$, and the stopcock being turned, the steam cools and is condensed, and water is forced into its place by the pressure of the atmosphere, through the valve $\mathrm{C}$ : the steam is then readmitted, and forces the water to ascend through the valve $D$ and the pipe $D E$. The vessel $F$ acts alternately with $A$. P. 266.

Fig. 336. The common steam engine of Newcomen and Beighton. The steam being admitted into the cylinder $A$ below the piston, the weight $B$ is allowed to descend : a jet of water is then admitted by the pipe $C$, which condenses the steam, and the pressure of the atmosphere then depresses the piston: a part of this water is admitted by the pipe D into the boiler, in order to keep it sufficiently full. P. 266 .

Fig. 337. Mr. Watt's steam engine. The steam, which is below the piston, is suffered to escape into the condenser $A$ by the cock $B$, which is opened by the rod C, and at the same time the steam is admitted by the cock D into the upper part of the cylinder; when the piston has descended, the cocks $\mathrm{E}$ and $\mathrm{F}$ act in a similar manner in letting out the steam from above and admitting it below the piston. The 
jet is supplied by the water of the cistern $G$, which is pumped up at $\mathbf{H}$ from a reservoir : it is drawn out, together with the air that is extricated from it by the air pump I, which throws it into the cistern $K$, whence the pump $\mathbf{L}$ raises it to the cistern $\mathbf{M}$; and it enters the boiler through a valve, which opens whenever the float $\mathrm{N}$ descends below its proper place. The pipes $O$ and $\mathbf{P}$ serve also to ascertain the quantity of water in the boiler. The piston rod is confined to a motion nearly rectilinear by the frame $Q$; the fly wheel $R$ is turned by the sun and planet wheel $S, T$; and the strap $\mathrm{U}$ turns the centrifugal regulator $\mathrm{W}$, which governs the supply of steam by the valve or stopcock X. P. 267.

Fig. 338. Mr. Symington's steam boat. A is the boiler, B the cylinder, C the piston, $\mathbf{D}$ the condensation pipe, $\mathbf{E}$ the air pump, $\mathbf{F}$ stampers for breaking ice. P. 267.

Fig. 339. An air gun. The air is forced by the syringe A into the cavity surrounding the barrel, whence it is discharged by the valve $B$, which is opened either immediately by the action of the trigger $\mathrm{C}$, or by a spring, which is bent by cocking the gun, and set at liberty by the trigger. P. 269.

\section{PLATE XXV.}

Fig. 340. A series of waves or pulses of sound, diverging from one of the foci of an ellipsis, and reflected towards the other. P. 293.

Fig. 341. Waves diverging from a point near the centre of a circle, and converg. ing after reflection to a point at an equal distance on the other side of the centre. P. 293.

Fig. 342. A section of a speaking trumpet and of a hearing trumpet : the lines representing the direction of the sound before and after its reflections. P. 294 .

Fig. 343. A string impelled by the bow of a violin, and lightly touched at the same time at a point one third of its length from the end : the small pieces of paper fly off from the middle of the vibrating portions, while the piece situated at the remaining point of division retains its situation. P. 299.

Fig. 344. A vibration compounded with another smaller vibration, three times as frequent, in a transverse direction, the separate vibrations being such that the points may be always opposite to a point moving uniformly in a circle. Thus the vibrations in the lines A B and A C compose the complicated figure D E. P. 299.

Fig. 345. A specimen of the manner in which the vibrations of a string are usually performed when it is struck with a bow. P. 299.

Fig. 346. Specimens of the simplest manner in which sand is collected into lines, on a plate of glass or metal, which is made to sound by means of the bow of a violin. P. 300 .

Fig. 347. A round plate, performing some of its most complicated vibrations, the lines of division being indicated by the place of the sand. From Chladni. P. 300 .

Fig. 348. A square plate divided into a diversity of vibrating portions. From Chladni. P. 300 .

Fig. 349. The small bones of the left ear, nearly three times the natural size, supposed to be seen through the membrane of the tympanum, by looking directly into the auditory canal. A B is the membrane of the tympanum, $\mathrm{C}$ the hammer, D the anvil, $\mathrm{E}$ its attachment to the surrounding bone, $\mathrm{F}$ the stirrup, $\mathrm{G}$ the round aperture in the bone leading to the cochlea. P. 302.

Fig. 350. A view of the vestibule of the left ear, with the semicircular canals and the cochlea, seen with the eye a little more depressed than by looking straight through the canal, and exactly in the direction of the stirrup. A B C is the vestibule, immediately behind the oval aperture, which is covered by the basis of the stirrup, $D$ are the canals, $\mathrm{E}$ the cochlea, the upper spire terminating in the vestibule, the lower in the round aperture at $B$. The projection of the membrane of the tympanum is marked by an oval line. P. 302.

Fig. 351. The structure of the left ear, seen from above, the upper part of the canal being supposed to be removed. $A$ is the auditory canal, $B$ the membrane of the tympanum, $\mathrm{C}$ the hammer, D the anvil, $\mathbf{E}$ the stirrup ; $\mathrm{F}$ the place of the canals, 
which are higher than the parts represented, $\mathrm{G}$ the place of the cochlea, $\mathrm{H}$ the round aperture. P. 302 .

Fig. 352. A, B, C, a representation of the joint effect of two equal vibrations variously combined, the middle line being always half way between the two outer ones, and showing the compound vibration reduced to half its real extent: D shows the mode of finding the joint effect of vibrations, by cutting a surface into sliders, which are retained in their places by a screw. P. 305 .

Fig. 353. The uppermost and lowermost curves represent a series of vibrations, of which 12 occupy any given period of time : the third and sixth lines two series of which 15 and 16 occupy respectively the same time : the joint effect of each pair is shown by the dotted curves which are interposed between them, the middle one representing the effect denominated a beat. P. 305, 306 .

Fig. 354. The proportional lengths of a chord or pipe, constituting the different notes of the simple diatonic scale, with their mutual relations, shown by their divisions into aliquot parts. P. 307.

Fig. 355. A good practical mode of temperament; making all the fifths and the third in the first division perfect concords; the three remaining fifths equally imperfect. P. 309.

Fig. 356. The trumpet of Marigni, with its bridge, which is supported by the string A B nearly in contact with the sounding board; this string being either stretched by a pin at B, or by a cross string B C. The places at which the string is to be touched, may be marked by frets fixed under them, as they are here shown by points. At $\mathrm{D}$, the scale of this instrument is exhibited, resembling that of the trumpet and the French horn. P. 312.

\section{PLATE XXVI.}

Fig.357. The right half of the human larynx. A B C is the outline of the cricoid cartilage, DEFG $\mathrm{H}$ of the thyreoid, and I KL of the arytaenoid cartilage; $\mathrm{M}$ is the epiglottis, N K the upper ligament of the glottis, O P the lower ligament, and $Q$ the trachea. P. 313 .

Fig. 358. A view of the ligaments of the glottis, seen from above, the larynx being divided by a horizontal section a little above them. P. 313 .

Fig. 359. Sections of the pipes employed by Kratzenstein for producing the sounds of the different vowels; in general by means of a larynx resembling the mouth piece of a reed organ pipe, but in the case of the vowel I by simple inflation through the tube B. The pipe for $U$ produces the sound $O$, exeept when it is very nearly shut up. P. 313.

Fig. 360. The vox humana organ pipe, with the mouth piece common to reed pipes in general; the lower part in contact with the tongue being nearly semicylindrical ; the tongue being adjusted to the proper pitch by means of a sliding wire, which regulates the length of the part that is at liberty to vibrate. P. 314 .

Fig. 361. The mouth piece proposed by Kratzenstein, for imitating the human voice, the tongue A passing freely in and out of the tube, which is more than half of a cylinder, as is seen at B. P. 314 .

Fig. 362. The form of the regal organ pipe. P. 314 .

Fig. 363. A front view and section of the open diapason organ pipe of metal. It is tuned by opening or contracting the upper orifice. P. 314 .

Fig. 364. A a front view of the flute organ pipe, of wood, which is tuned by a plug. B a section of the pipe. P. 314 .

Fig. 365. A stopped diapason organ pipe, of metal. It is tuned by altering the position of the pieces on each side of the mouth. P. 314 .

Fig. 366. A chimney pipe. P. 314 .

Fig. 367. A spindle shaped organ pipe, contracted above. P. 314.

Fig. 368. A the form of a cromorn pipe, B, of a trumpet pipe, both having reed mouth pieces. P. 315 .

Fig. 369. A ray or pencil of light A B, C B, falling on the surface D E; a portion of the light is reflected, and another portion is transmitted, in the direction $\mathrm{BF}, \mathrm{BG}$, so that $\mathrm{BG}$ is equal to $\mathrm{BC}$, and $\mathrm{BH}$ to $\mathrm{BI}, \mathrm{C} \mathrm{IK}$ and $\mathrm{GH} \mathrm{L}$ being 
lines perpendicular to $\mathrm{DE}$ at any such distances, that $\mathrm{B} \mathrm{K}$ may be to $\mathrm{B} \mathrm{L}$ in a certain proportion, which is that of the sines of the angles of incidence A B M, C B M, to those of the angles of refraction $\mathrm{FBN}, \mathrm{GBN}$. BO and B P are the reflected portions of the rays. P. 322 .

Fig. 370. A mode of determining the position of a refracted ray, which is particularly convenient in the case of refractions at spherical surfaces. A B C being any circle, either touching the refractive surface at A, or being itself a section of the refracting substance, if another circle DE F be drawn on the same centre, having its diameter to that of the first as the sine of the angle of incidence to that of refraction, and a third circle GH I, which is less than the first in the same proportion as the second is greater; and if the direction of the incident ray $\mathrm{KA}$ be continued to $\mathrm{D}$, and $\mathrm{LD}$ be drawn from the centre cutting G H I in G, A G will be the direction of the refracted ray; and if this ray pass again out of the denser medium at $B$, its direction B M may be found by drawing L I F, and F B M will be thus truly determined. P. 322.

Fig. 371. A ray or pencil A B, refracted at B to C, and there reflected by a perpendicular surface into an opposite direction $\mathrm{C} \mathrm{B}$, will return also in the direction $\mathrm{B} A$, a portion of it being reflected in the first place to $\mathrm{D}$, and in the second to $\mathrm{E}$. P. 323.

Fig. 372. A pencil A B passing through a substance CD contained between parallel surfaces, continues its course in the direction EF parallel to A B. P. 324 .

Fig. 373. The ray $\mathbf{A B}$, entering the medium $\mathbf{C D}$ through the transparent substance E F, contained between parallel surfaces, acquires the direction $\mathrm{GH}$, parallel to $I K$, into which $L I$ is at once refracted. P. 324 .

Fig. 374. The appearance of a prism, of which the lower surface is divided into a bright and a dark portion, separated by a coloured arch ABC. P. 324 .

\section{P L A T E XXVII.}

Fig. 375. $\mathrm{A}$ is an actual focus of diverging rays, $\mathrm{B}$ an actual focus both of converging and of diverging rays, $C$ a virtual focus of converging rays, and $D$ a virtual focus of diverging rays; $\mathrm{A}$ and $\mathrm{B}, \mathrm{B}$ and $\mathrm{C}$, and $\mathrm{C}$ and $\mathrm{D}$ are foci conjugate to each other, with respect to the refractions of the three lenses. P. 325 .

Fig. 376. The image of the point $N$, formed by the plane mirror AB, is at an equal distance behind the mirror; and in this manner the whole image of the word is formed in an inverted position. P. 325 .

Fig. 377. A B CD represents a pencil of parallel rays falling on the concave mirror $\mathrm{CD}$, and collected into the principal focus at $\mathbf{E}$, which is half way between the surface and its centre. $F$ is the principal focus of the convex mirror $G$; and $H$ that of the refracting surface $I$. P. 326 .

Fig. 378. A being the centre of the concave mirror B, the image of an object at $C$ will be found at $D$, and the reverse. P. 326 .

Fig. 379. A pencil of light, deflected from its path by a prism of a denser substance, in different positions. P.326.

Fig. 380. A pencil of light scattered into various directions by a multiplying glass. P. 326.

Fig. 381. $\mathbf{A}$ is a section of a double convex lens, $\mathbf{B}$ of a double concave. $\mathbf{C}$ is a planoconvex, D a planoconcave; and $\mathrm{E}$ and $\mathrm{F}$ meniscus lenses; but a meniscus of the form represented by $\mathrm{F}$ is sometimes called a concavoconvex lens. P. 326 .

Fig. 382. The pencils of light A, B are refracted by the convex lens $\mathbf{C}$ in the same manner as they would have been by the circumscribed double prism $\mathbf{D E}$; and in the same manner the concave lens $\mathbf{F}$ resembles in its operation the prisms $\mathbf{G}, \mathbf{H}$. P. 326.

Fig. 383. A, a pencil of parallel rays, made to converge, by a double convex lens of crown glass, to the centre of curvature of one of its surfaces. B a double con- 
cave lens, causing the rays to diverge from the centre of curvature. C, D a planoconvex lens, of which the principal focus is at the distance of a diameter. P. 326 .

Fig. 384. The lenses represented by the shaded surfaces are equivalent in their effects to those of which the sections are shown by the dotted lines; the figures at $\mathbf{A}$ and $\mathrm{B}$ being of equal thickness in the middle, and at $\mathrm{C}$ at the edges also. P. 326.

Fig. 385. At A, a radiant point and its image are both situated at the distance of twice the focal length from the lens; at $\mathrm{B}$, the one is more remote, the other nearer; and $C D$ is to $D E$ as $E F$ to FG; D and F being the principal foci of the lens. P. 327 .

Fig. 386. The oblique pencils of rays $\mathrm{A}, \mathrm{B}$, and the direct pencil $\mathrm{C}$, are supposed to be brought to their respective foci in the same plane D E. P. 327 .

Fig. 387. The square $\mathbf{A}$ intercepts the whole light, proceeding from the point B, which would fall on the surface $C D$, four times as great, placed at a double distance. P. 329 .

Fig. 388. The box of Count Rumford's photometer. The lights, being placed at proper distances on the graduated arms or tables A, B, throw equally dark shadows of the cylinders C, D on a white surface at E F. The wings of the cylinders serve to make the shadows of equal breadth. The shadows are viewed through the aperture at G. P. 329.

Fig. 389. Dr. Wollaston's instrument for the measurement of refractive densities. A is a rectangular prism of flint glass, under which the substance to be examined is attached; BC is a rod, or ruler, 10 inches long, CD and DE are each 15 100 . When the sights at $\mathrm{B}$ and $\mathrm{C}$ are so placed that the division between the light and dark portion of the lower surface of the prism is seen through them, the rod $F$, which carries a vernier, shows the index of the refractive density, which, in the situation here represented, would be 1.43. P. 329 .

Fig. 390. A is the actual image of the candle B, formed by the convex lens $\mathbf{C}$. P. 330 .

Fig. 391. $\mathrm{A}$ is the actual image of the candle $\mathrm{B}$, formed by the concave mirror C. P. 330 .

Fig. 392. A is the actual image of the candle B, formed by the convex lens $\mathbf{C}$, being as much larger than the object as it is more distant from the lens. P. 330 .

Fig. 393. A is the virtual image of the candle $B$, placed within the focal distance of the concave mirror $C$, the image remaining erect. P. 330 .

Fig. 394. A is the virtual image of the candle B, formed by the concave lens $\mathbf{C}$, and less than the object. P. 330 .

Fig. 395. When the object $\mathrm{A}$ is placed in the principal focus of the convex lens $B$, a virtual image is formed at an infinite distance, which subtends, when viewed from $\mathrm{C}$, or from any other point, the same angle as the object subtends at the centre of the lens. P. 330 .

Fig. 396. The object A being placed a little within the focus of the lens B, a virtual image $\mathbf{C}$ is formed, at such a distance as is most convenient to the eye, which subtends the same angle as the object, from the centre of the lens, and therefore appears somewhat more magnified than when the object is in the principal focus. P. 330 . 


\section{PLATE XXVIII.}

Fig. 397. An imperfect image of an external object, painted in a dark room, in an inverted position, by the light coming in right lines through a small aperture. P. 332.

Fig. 398. A portable camera obscura. A is a lens, B a mirror placed obliquely, and throwing the image on a plate of ground glass, C D. E is a moveable cover, and F G a screen attached to it, for excluding foreign light. P. 332.

Fig. 399. A camera obscura, which throws down an image, by means of the mirror $\mathbf{A}$, and the lens $\mathbf{B}$, on the surface $\mathbf{C}$, where it may be seen through the aperture $\mathrm{D}$. The surface $\mathrm{C}$ has here the curvature best adapted to receive every where a perfect image of a distant object. P. 332 .

Fig. 400. An arrangement proposed for a solar microscope, adapted to a window facing the south. The mirror $\mathrm{A}$ is moved by a hinge into the position required for the day, and during the employment of the instrument is turned only round the axis $A \mathrm{~B}$, which is parallel to that of the earth. The mirror $\mathrm{C}$ is fixed : it receives the beam of light from $\mathbf{A}$, and throws it on the object through the lenses $\mathbf{D}$ and $\mathbf{E}$, of which the joint focus is near the magnifying lens $\mathrm{F}$; this lens paints an image of the object in an inverted position on a screen at $G$. If the focus of the condensing lenses were behind the object, as at $\mathrm{H}$, the light would be liable to be condensed into a spot on the screen at I. P. 333 .

Fig. 401. An arrangement proposed for a phantasmagoria. The light of the lamp $A$ is thrown by the mirror $B$ and the lenses $C$ and $D$ on the painted slider at $\mathbf{E}$, and the magnifier $\mathbf{F}$ forms the image on the screen at $\mathbf{G}$. This lens is fixed to a slider, which may be drawn out of the general support or box $\mathrm{H}$ : and when the box is drawn back on its wheels, the rod I K lowers the point $K$, and by means of the rod $\mathrm{KL}$ adjusts the slider in such a manner, that the image is always distinctly painted on the screen G. When the box advances towards the screen, in order that the images may be diminished and appear to vanish, the support of the lens $\mathrm{F}$ suffers the screen $\mathrm{M}$ to fall and intercept a part of the light. The rod $\mathrm{K} N$ must be equal to $I K$, and the point I must be twice the focal length of the lens $F$, before the object, $\mathbf{L}$ being immediately under the focus of the lens. The screen $\mathbf{M}$ may have a triangular opening, so as to uncover the middle of the lens only, or the light may be intercepted in any other manner. P. 334.

Fig. 402. The construction of the astronomical telescope. A BC and DEC are the central parts of the pencils of rays, coming, from the extremities of the visible field, through the middle of the object glass. P. 334 .

Fig. 403. The extreme pencils of rays in the double or compound microscope. P. 334.

Fig. 404. The extreme pencils in the Galilean telescope, or opera glass. P. 334 .

Fig. 405. A, the directions of the extreme pencils in the common day telescope of Rheita. If only two eye glasses were employed, as at B, the field would obviously be more contracted. P. 334 .

Fig. 406. Dr. Herschel's forty feet telescope. ABC the path of a ray of light, reflected by the mirror at $B$ to the eye glass $C$. D a chair in which the observer sits. E a moveable gallery, on which several persons may stand. FG a smooth surface, on which the bottom of the telescope is made to roll along, while its opening is raised or depressed by the pullies at $H$ and $I$. $K$ one of two rooms or huts for the accommodation of the observer's assistants. The wheels, under the frame, serve to turn the whole instrument round its centre. P. 335 .

Fig. 407. The Newtonian telescope, with the direction of the central rays. These are not the rays by which the object is actually seen, because they are intercepted by the small speculum, but they afford the simplest determination of the magnitude of the field of view. P. 335 .

Fig. 408. The supposed path of the central rays in the Gregorian telescope. P. 335 .

Fig. 409. The supposed path of the central rays in Cassegrain's telescope. Here the rays actually represented would not only be intercepted by the small mirror, but 
which are higher than the parts represented, $\mathrm{G}$ the place of the cochlea, $\mathrm{H}$ the round aperture. P. 302.

Fig. 352. A, B, C, a representation of the joint effect of two equal vibrations variously combined, the middle line being always half way between the two outer ones, and showing the compound vibration reduced to half its real extent: D shows the mode of finding the joint effect of vibrations, by cutting a surface into sliders, which are retained in their places by a screw. P. 305 .

Fig. 353. The uppermost and lowermost curves represent a series of vibrations, of which 12 occupy any given period of time : the third and sixth lines two series of which 15 and 16 occupy respectively the same time : the joint effect of each pair is shown by the dotted curves which are interposed between them, the middle one representing the effect denominated a beat. P. 305,306 .

Fig. 354. The proportional lengths of a chord or pipe, constituting the different notes of the simple diatonic scale, with their mutual relations, shown by their divisions into aliquot parts. P. 307.

Fig. 355. A good practical mode of temperament; making all the fifths and the third in the first division perfect concords ; the three remaining fifths equally imperfect. P. 309.

Fig. 356. The trumpet of Marigni, with its bridge, which is supported by the string A B nearly in contact with the sounding board; this string being either stretched by a pin at B, or by a cross string B C. The places at which the string is to be touched, may be marked by frets fixed under them, as they are here shown by points. At $D$, the scale of this instrument is exhibited, resembling that of the trumpet and the French horn. P. 312.

\section{PLATE XXVI.}

Fig.357. The right half of the human larynx. A B C is the outline of the cricoid cartilage, DE F G H of the thyreoid, and I KL of the arytaenoid cartilage; $\mathrm{M}$ is the epiglottis, N K the upper ligament of the glottis, O P the lower ligament, and $Q$ the trachea. P. 313 .

Fig. 358. A view of the ligaments of the glottis, seen from above, the larynx being divided by a horizontal section a little above them. P. 313 .

Fig. 359. Sections of the pipes employed by Kratzenstein for producing the sounds of the different vowels; in general by means of a larynx resembling the mouth piece of a reed organ pipe, but in the case of the vowel I by simple inflation through the tube $B$. The pipe for $U$ produces the sound $O$, exeept when it is very nearly shut up. P. 313 .

Fig. 360. The vox humana organ pipe, with the mouth piece common to reed pipes in general ; the lower part in contact with the tongue being nearly semicylindrical; the tongue being adjusted to the proper pitch by means of a sliding wire, which regulates the length of the part that is at liberty to vibrate. P. 314 .

Fig. 361. The mouth piece proposed by Kratzenstein, for imitating the human voice, the tongue A passing freely in and out of the tube, which is more than half of a cylinder, as is seen at B. P. 314 .

Fig. 362. The form of the regal organ pipe. P. 314 .

Fig. 363. A front view and section of the open diapason organ pipe of metal. It is tuned by opening or contracting the upper orifice. P. 314 .

Fig. 364. A a front view of the flute organ pipe, of wood, which is tuned by a plug. B a section of the pipe. P. 314 .

Fig. 365. A stopped diapason organ pipe, of metal. It is tuned by altering the position of the pieces on each side of the mouth. P. 314 .

Fig. 366. A chimney pipe. P. 314 .

Fig. 367. A spindle shaped organ pipe, contracted above. P. 314 .

Fig. 368. A the form of a cromorn pipe, B, of a trumpet pipe, both having reed mouth pieces. P. 315 .

Fig. 369. A ray or pencil of light A B, C B, falling on the surface D E; a portion of the light is reflected, and another portion is transmitted, in the direction $\mathrm{BF}, \mathrm{BG}$, so that $\mathrm{BG}$ is equal to $\mathrm{BC}$, and $\mathrm{BH}$ to $\mathrm{BI}, \mathrm{C} \mathrm{I} \mathrm{K}$ and $\mathrm{G} \mathrm{H} \mathrm{L}$ being 
lines perpendicular to D E at any such distances, that B $\mathrm{K}$ may be to $\mathrm{B} \mathrm{L}$ in a certain proportion, which is that of the sines of the angles of incidence A B M, C B M, to those of the angles of refraction F BN, GBN. B O and B P are the reflected portions of the rays. P. 322 .

Fig. 370. A mode of determining the position of a refracted ray, which is particularly convenient in the case of refractions at spherical surfaces. A B C being any circle, either tonching the refractive surface at A, or being itself a section of the refracting substance, if another circle DE F be drawn on the same centre, having its diameter to that of the first as the sine of the angle of incidence to that of refraction, and a third circle GH I, which is less than the first in the same proportion as the second is greater; and if the direction of the incident ray $\mathrm{KA}$ be continued to $\mathrm{D}$, and $L D$ be drawn from the centre cutting GH I in G, A G will be the direction of the refracted ray; and if this ray pass again out of the denser medium at $B$, its direction B M may be found by drawing L I F, and F B M will be thus truly determined. P. 322.

Fig. 371. A ray or pencil A B, refracted at B to C, and there reflected by a perperidicular surface into an opposite direction $\mathbf{C ~ B}$, will return also in the direction $\mathrm{BA}$, a portion of it being reflected in the first place to $\mathrm{D}$, and in the second to $\mathrm{E}$. P. 323.

Fig. 372. A pencil A B passing through a substance CD contained between parallel surfaces, continues its course in the direction EF parallel to A B. P. 324 .

Fig. 373. The ray $A B$, entering the medium $C D$ through the transparent substance E F, contained between parallel surfaces, acquires the direction $\mathbf{G H}$, parallel to $I K$, into which $L I$ is at once refracted. P. 324 .

Fig. 374. The appearance of a prism, of which the lower surface is divided into a bright and a dark portion, separated by a coloured arch ABC. P. 324 .

\section{P L A T E XXVII.}

Fig. 375. A is an actual focus of diverging rays, $B$ an actual focus both of converging and of diverging rays, $C$ a virtual focus of converging rays, and $D$ a virtual focns of diverging rays; $A$ and $B, B$ and $C$, and $C$ and $D$ are foci conjugate to each other, with respect to the refractions of the three lenses. P. 325 .

Fig. 376. The image of the point $N$, formed by the plane mirror AB, is at an equal distance behind the mirror; and in this manner the whole image of the word is formed in an inverted position. P. 325 .

Fig. 377. A B CD represents a pencil of parallel rays falling on the concave mirror $\mathrm{CD}$, and collected into the principal focus at $\mathrm{E}$, which is half way between the surface and its centre. $F$ is the principal focus of the convex mirror $G$; and $\mathbf{H}$ that of the refracting surface $I$. P. 326 .

Fig. 378. A being the centre of the concave mirror B, the image of an object at $C$ will be found at D, and the reverse. P. 326 .

Fig. 379. A pencil of light, deflected from its path by a prism of a denser substance, in different positions. P.326.

Fig. 380. A pencil of light scattered into various directions by a multiplying glass. P. 326 .

Fig. 381. A is a section of a double convex lens, $B$ of a double concave. $C$ is a planoconvex, D a planoconcave; and $\mathrm{E}$ and $\mathrm{F}$ meniscus lenses; but a meniscus of the form represented by $F$ is sometimes called a concavoconvex lens. P. 326 .

Fig. 382. The pencils of light A, B are refracted by the convex lens $\mathbf{C}$ in the same manner as they would have been by the circumscribed double prism $\mathbf{D E}$; and in the same manner the concave lens $\mathbf{F}$ resembles in its operation the prisms $\mathbf{G}, \mathbf{H}$. P. 326.

Fig. 383. A, a pencil of parallel rays, made to converge, by a double convex lens of crown glass, to the centre of curvature of one of its surfaces. B a double con- 


\section{PLATE XXX.}

Fig. 436. A section of the human eye. $A$ is the cornea ; $B$ the aqueous humour, in which the uvea hangs; $C$ the crystalline lens; the ciliary processes being between it and the urea; D the vitreous humour; E F G is the choroid coat, lined by the retina; H IK the sclerotica, and L the optic nerve. P. 350.

Fig. 437. A picture painted on the retina in an inverted position, seen by dissecting off the sclerotica and choroid behind it. P. 351 .

Fig. 438. The apparent figure of the heavens being nearly like the curve A B C, the sun or moon at $\mathrm{A}$ or $\mathrm{C}$ appears to be much larger than at B. P. 356 .

Fig. 439. The red square A, inclosing a green square, produces, if viewed attentively, in a strong light, a spectrum resembling $\mathbf{B}$, which is red within and green without, and which appears when we look soon after on any white object. P. 357.

Fig. 440. The spot, which is tinted with black lines only, appears, upon the yellow ground, of a purple hue. P. 357 .

Fig. 441. A grey spot on a purple ground appears of a greenish yellow or olive hue. P. 357 .

Fig. 442. The manner in which two portions of coloured light, admitted through two small apertures, produce light and dark stripes or fringes by their interference, proceeding in the form of hyperbolas; the middle ones are however usually a little dilated, as at A. P. 365 .

Fig. 443. A series of stripes of all colours, of their appropriate breadths, placed side by side in the manner in which they would be separated by refraction, and combined together so as to form the fringes of colours below them, beginning from white. P. 365 .

Fig. 444. A series of coronae, seen round the sun or moon. P. 366 .

Fig. 445. The internal hyperbolic fringes of a rectangular shadow. $P .367$.

Fig. 446. The external fringes seen on each side of the shadow of a hair or wire, which is also divided by its internal fringes. The dotted lines show the natural magnitude of the shadow, independently of diffraction. P. 367 .

Fig. 447. Analysis of the colours of thin plates seen by reflection, beginning from black. A line drawn across the curved fringes wonld show the portions into which the light of any part is divided when viewed through a prism. P. 368 .

Fig. 448. The coloured stripes of a film of soapy water, covering a wine glass. P. 368.

Fig. 449. The colours of a thin plate of air or water contained between a convex and a plane glass, as seen by reflection. P. 368 .

Fig. 450. The colours of a mixed plate; as seen by partially greasing a lens a little convex, and a flat glass, and holding them together between the eye and the edge of a dark object. One half of the series begins from white, the other from black, and each colour is the contrast to that of the opposite half of the ring. P. 369 .

Fig. 451. The composition of the colours of the primary rainbow, when attended by supernumerary bows. P. 369 .

Fig. 452. The colours of concave mirrors. The small circles in the middle white ring represent the aperture by which the light is admitted, and its image; the coloured rings are formed by the light irregularly dissipated before and after reflection. P. 370 . 


\section{PLATE XXXI.}

Fig. 453, 454. The appearance of the star Lyra, viewed with telescopes magnifying 460 and 6450 times respectively. From Dr. Herschel. P. 390.

Fig. 455. The appearance of the nebula in Orion, about half a degree in length. From Messier.* P. 391.

Fig. 456.. 463. The appearances of different nebulae. From Dr. Herschel. P. 391 .

Fig. 464. A section of the nebula to which the sun is supposed to belong, its projection forming the milky way; taken in a plane perpendicular to its longest diameter. From Dr. Herschel. The large star in the middle represents the sun, and the circle drawn round it is at forty times the distance of the nearest fixed stars, comprehending probably all the stars which are visible to the naked eye. P. 362 .

Fig. 465. A large spot, traced through different forms in its path across the sun. From Dr. Wilson. A is its place 23 Nov. $1769 ; \mathrm{B}, 24$ Nov.; C, 11 Dec.; D, 12 Dec.; and E, 17 Dec. P. 399.

Fig. 466. A, a large spot on the sun; B, the arrangement of the luminous and opaque strata of clouds by which Dr. Herschel explains the appearance of the spot. P. 399.

Fig. 467. A, a spot with a lighter portion in the middle; $B$, the arrangement of the strata corresponding to it. P. 399.

Fig. 468. The position assumed by the strata which had formed the spot shown in the last figure, viewed about an hour afterwards. P. 399.

Fig. 469. A and B are the forms of a solar spot, at about two hours' distance of time; C, D, and E, are the successive forms of another spot. P. 399.

Fig. 470. The appearance of the zodiacal light, or solar atmosphere, as it is seen in these climates, in the evening, about the beginning of March; A B being the horizon, and C the supposed place of the sun. P. 400 .

\section{P L A T E XXXII.}

Fig. 471. A representing the sun, B the earth, and C the planet Mars; supposing Mars and the earth to set out together from $\mathbf{D}$ and $\mathbf{E}$, the angle $\mathbf{D} \mathbf{A} \mathbf{C}$ was determined by Kepler from calculation, and the angles B A D and A B C by observation; whence it was easy to construct the triangle A B C, and to find the proportion of $\mathrm{A} \mathrm{B}$ to A C. P. 402 .

Fig. 472. The solar system, representing the form and proportions of the orbits of all the primary planets, and of three of the comets. The parts of the orbits represented by entire lines are on the north of the ecliptic, the dotted parts on the south : the letters $\mathrm{A}$ and $\mathrm{P}$ denote the aphelion and perihelion. The point in the centre, which ought to be only $\frac{1}{700}$ of an inch in diameter, represents the sun. The figures of the respective planets show their comparative magnitude, that of the sun being represented by the innermost of the graduated circles which inclose the whole : they are placed according to their actual situations on the 14th June, 1806. The letters MD show the mean distance of the comet of 1759 , being placed at the extremity of the lesser axis of the ellipsis in which it must be supposed to revolve. P. 408.

Fig. 473. The periodical times of the different planets, represented by lines of different lengths. P.408.

Fig. 474. The comparative velocities of the different planets, represented by lines which show the number of English miles described in a second, on the scale marked on the lowest line. P. 408.

Fig. 475. The places of the ascending nodes of all the planets, marked on one half of the ecliptic, supposed to be extended in a straight line; together with the inclinations of their orbits. The line marked F. F. E. E, shows the situation of the fixed ecliptic. P. 408.

* Hist. et Mém. 1771, p. 458. 


\section{P LATE XXXIII.}

Fig. 476. A. The appearance of Venus, from Dr. Herschel : B, C, from Mr. Schroeter. P. 408.

Fig. 477. A..D, the appearance of Mars, from Dr. Herschel. The figures are inverted, as they appear in the astronomical telescope. P. 408.

Fig. 478. A, B. The appearance of Jupiter, with his belts, from Dr. Herschel. P. 408 .

Fig. 479. The appearance of Saturn, with his ring, from Dr. Herschel. P. 408.

Fig. 480. The appearance of the moon, in an inverted position. The figure is copied from Mr. Nicholson's plate, the references from Cassini and Lalande. Eq. is the place of the moon's equator. P. 408.

Names of the spots, according to Riccioli, and Hevelius.

1 Grimaldus or Palus Mareotis

2 Galileus Mons Audus

3 Aristarchus

4 Keplerus

5 Gassendus

6 Schikardus

7 Harpalus

8 Heraclides

(b) Vulcanus

9 Lansbergius

10 Reinoldus

11 Copernicus

12 Helicon

13 Capuanus

14 Bullialdus

15 Eratosthenes

16 Timocharis

17 Plato

18 Archimedes

(a) Aratus

19 Insula sinus medii

20 Pitatus

21 Tycho

22 Eudoxus

23 Aristoteles

24 Manilius

25 Menelaus
Mons Porphyrites

Loca paludosa

Mons Cataractes

Mons Troicus

Insula sinus hyperborei

Caput mulieris

Insula Malta

Mons Neptunus

Mons Aetna

Insula erroris

Regio Cassiotis

Insula Creta

Insula Vulcania

Insula Corsica

Locus niger major

\section{Mare mortuum}

Mons Sinai

Mons Carpathes

Mons Serrorum

Insula Berbicus

Byzantium
Mons Bodinus

26 Hermes

27 Dionysius

(d) Albategnins

29 Plinius

$30 \mathrm{~S}$. Theophilns

31 Fracastorius

32 Censorinus

33 Messala

34

35 Proclus

36 Cleomedes

37 Snellius

38 Petavius

39 Langrenus

40 Taruntius

Promontorium Acherusia

Mons Moschi

Lacus Thospitis

Promontorium acutum

Promontorium Somnii

Mous Corax

Montes Riphaei

Mons Paropamisus

Petra Sogdiana

Insula major

Sinus Phasianus

Fig. 481..483. The satellites of Jupiter, Saturn, and the Georgian planet, at their proper distances, in proportion to the diameters of the planets, shown on the same scale. P. 408.

Fig. 484. The figure of the tail of the comet of 1680 , represented in the plane of its orbit, from Newton. A B is the earth's orbit, C and D are the first and last appearances of the tail, and E F is the line of the nodes. P.408.

Fig. 485. A, B. Two successive appearances of the comet of 1723 , from Lord Paisley. P. 408. 


\section{P LA T E XXXIV.}

Fig. 486. The gravitating body A B C, being supposed to revolve on the axis A C, the fluid column BD must be longer than $\mathbf{E}$, in order to support its pressure. P. 412 .

Fig. 487. If $A$ represent the place of the sun, $B$ that of the earth, and $C$ that of the moon, taking $\mathrm{AD}$ to $\mathrm{AC}$ as the square of $\mathrm{AC}$ is to the square of $\mathrm{AB}, \mathrm{AD}$ will represent the sun's attraction acting on the earth, and C D the disturbing force, which, together with A D, makes up A C, the force acting on the moon; and it is obvious that, when the nodes are in any oblique situation, as E F, the force being directed to some point $D$, between $B$ and $A$, while the moon moves from $G$ to $H$, the force $\mathrm{CD}$ will tend to lessen the inclination, while the moon is ascending from $\mathbf{E}$ towards $\mathbf{C}$, and to cause the node $\mathbf{E}$ to move back towards $\mathbf{G}$, and, when it is again descending towards $\mathrm{F}$, the inclination will be increased, and the node $\mathrm{F}$ made to recede towards $\mathbf{H}$, nntil the moon arrives at $\mathbf{H}$, and the force becomes directed to a point on the other side of $\mathrm{B}$; the nodes only advancing while the moon is between $\mathrm{H}$ and $\mathrm{F}$, or between $\mathrm{G}$ and $\mathrm{E}$. P. 413 .

Fig. 488. A body attracted towards the centre $A$, and descending from $B$ in the ellipsis $\mathrm{B} \mathrm{C} \mathrm{D,} \mathrm{has} \mathrm{the} \mathrm{inclination} \mathrm{of} \mathrm{its} \mathrm{orbit} \mathrm{to} \mathrm{the} \mathrm{revolving} \mathrm{radius} \mathrm{A} \mathrm{B,} \mathrm{A} \mathrm{C,} \mathrm{A} \mathrm{D,}$ perpetually changed, until at $\mathrm{D}$ it becomes perpendicular to it : but when the force increases more rapidly, the radius does not become perpendicular to the orbit till it arrives at E, and the line of the apsides A D moves forwards to E. P.414.

Fig. 489. A represents the position of the limit of light and darkness on the earth's surface at the vernal equinox, $B$ at the summer solstice, and $C$ at the winter solstice; $\mathrm{EQ}$ denotes the equator, $\mathrm{N}$ the north pole, and $\mathrm{S}$ the south. P. 417.

Fig. 490. N E S W being the horizon, and Z the zenith, E A W shows the sun's apparent path in London at the time of the equinoxes, B C D at midsummer, and F G H at midwinter, projected orthographically, as if the circles were described on the surface of a globe, and viewed from a great distance. The circle I K L is the boundary of twilight, supposing it $18^{\circ}$ below the horizon, and its intersections with the sun's path show the beginning and end of twilight, as at I and K. P.418.

Fig. 491. The rays of light, coming in the direction $\mathrm{AB}$, are bent by the atmosphere so as to arrive at $\mathbf{C}$, and to illuminate a part of the atmosphere there, which is visible, by means of a second refraction, to a spectator at $\mathrm{D}$, and occasions the first and last twilight. P. 527.

Fig. 492. Venus is at her greatest elongation or angular distance from the sun $\mathbf{A}$, when situated as at $B$, with respect to the earth at $C$; and she is stationary at $D$, when she is moving with the same velocity as the earth, with respect to the direction of the earth's motion, the line E D being then more oblique, with respect to a fixed line, than either before or after. P.418.

Fig. 493. A B CD is the apparent path of Venus for the year 1806, supposing the sun $\mathrm{E}$ to revolve round the earth $\mathrm{F}$. The place of the sun and planet is marked for every four weeks. P. 418.

Fig. 494. The apparent path of Saturn in the heavens for the year 1806, referred to its proper place with respect to the ecliptic. The figures denote the places at the beginning of each month. P.418.

Fig. 495. The small figures represent the phases of the moon in different parts of her orbit. The smaller detached figures show the appearance of the moon, as seen from the earth; the larger ones, those of the earth at the same times, as seen from the moon, which are always the reverse of the moon's appearance. At $A$ the moon is new ; $B$ is the first quarter, $C$ the full moon, and $\mathbf{D}$ the last quarter. A and $C$ are sometimes called the syzygies, and $B$ and $D$ the quadratures. $P .419$.

Fig. 496. A, the moon passing through the earth's shadow; which is distinguished into three parts, the perfect shadow, the true shadow, and the penumbra. At $B$ and $C$ the moon is shown passing through the section of the shadow. P. 420.

Fig. 497. The path of the moon's shadow passing over the earth, in the solar eclipse of 1764, the earth being supposed at the same time to revolve on its axis. The line A B is the part in which the eclipse appeared annular, CD being the breadth of the whole shadow or penumbra. P.420.

Fig. 498. The shadow of the moon falling on the earth. The true shadow not 
extending here to the earth, the cone formed by the continuation of its outlines marks the extent of the parts in which the eclipse appears annular. P.420.

Fig. 499. The termination of the moon's disc in a solar eclipse. From Dr. Herschel. P. 420 .

Fig. 500. The apparent magnitudes of the planets, that of the sun or moon being supposed equal to a circle a foot in diameter: where there are two figures, one of them shows the mean apparent magnitude, and the other the greatest. P. 422 .

Fig. 501. The apparent magnitude of the sun, as seen from the different planets; for Mercury, the magnitude is shown by that of the earth in fig. 497 . P. 424 .

\section{P LATE XXXV.}

Fig. 502. A B being the earth's axis, the circle A C B is the meridian of the place $C$, and $C D$ represents the plane of its horizon. P. 426.

Fig. 503. The effect of the obliquity of the ecliptic in the equation of time is shown by the difference of the angles $\mathrm{ABC}$ and D B E, subtended at the pole B by equal portions of the oblique circle A E. P. 427.

Fig. 504. A B being parallel to the earth's axis, the 12 planes passing through it, at equal angular distances, mark, on the circle $C D$ perpendicular to it, the hour lines of an equatorial dial, and on the horizontal surface E F those of a horizontal dial. P.427.

Fig. 505. A method of constructing a dial on any given plane. A B C is the elevation of the pole, or more generally, the angle which the surface makes with the gnomon A B. The circles are divided into equal parts, and 1, 2, 3, 4, 5, 6 are the hour lines, $\mathrm{B}$ being the place of the gnomon. The reason of this construction will appear by comparing the circle in the last figure with the ellipsis which is formed on the horizontal surface. P. 427.

Fig. 506. A dial for a pointed gnomon, or obelisc, drawn on a horizontal surface. P. 427 .

Fig. 507. A mural quadrant, with its telescope ; A B is the plumb line, for adjusting the instrument, and $C$ the counterpoise for the telescope. P. 429.

Fig. 508. A portable transit instrument. $\mathbf{A}$ and $\mathbf{B}$ are screws for adjusting the axis by a vertical and a horizontal motion; C D is a spirit level, which may occasionally be hung on the telescope by the pins $\mathrm{E}$ and F. G is a small graduated arch, to be viewed through the microscope $\mathrm{H}$, for taking elevations of a few degrees. P. 429 .

Fig. 509. A transit circle, resembling Mr. Wollaston's, with a horizontal circle, by means of which both altitudes and azimuths may be measured. $A$ is a microscope for viewing the plumb line, B another for reading off the divisions of the horizontal circle; $\mathrm{C}$ and $\mathrm{D}$ are spirit levels. P. 429.

Fig. 510. A zenith sector, with its telescope, which has usually a reflecting prism, like that of the Newtonian telescope, for its eyeglass. P.429.

Fig. 511. The marine octant, introduced by Hadley. The mode of taking the common or front observation, is shown by the lines drawn to the sun and moon: the back observation by the two stars. A is a dark glass to be used in observations of the sun, and which may be fixed at B, when required. P.430.

Fig. 512. A B being the situation of the earth's axis, if the angle C B D, or the altitude of the body $\mathrm{D}$, be measured, and we subtract from it the elevation of the equinoctial C B E, the remainder will be the declination E B D. P. 430.

Fig. 513. The angle A B C is the moon's horizontal parallax, and D B C the parallax when she is elevated above the horizon D E in the angle B D E. P.430.

Fig. 514. The situation of the earth at the transit of Venus in June 1769. A spectator at the North Cape was carried during the transit from $\mathbf{A}$ to $\mathbf{B}$, and the transit appeared to him to last while Venus moved from $C$ to $D$ : the island of Otaheite, on the contrary, which is situated on the lower part of the illuminated hemisphere, was carried from $\mathrm{E}$ to $\mathrm{F}$, and the duration of the transit was there only while Venus moved from $G$ to $H$. Hence the rotatory motion of the earth was 
compared with the excess of the motion of Venus in its orbit above that of the earth. P. 431.

Fig. 515. A planisphere nearly resembling that of Professor Bode. The outer circle is fixed to the chart, and is divided either according to the degrees of the ecliptic, or the days of the month; the graduated circle immediately within it is divided into 24 hours, and is fixed to a circle of pasteboard, ont of which the circle NE S W, representing the horizon, is cut, the place being filled by thin varnished paper, with circles of azimnth and altitude engraved on it, which is carried round with the hour circle. P. 433.

Fig. 516. A diagram showing the length of the day, and the time of the sun's rising and setting in any part of the globe, within a few minutes; the time of the year being found in the graduated circle representing portions of the ecliptic, and the latitude on the middle line, by following the concentric circles of declination till they meet the horizon passing through the given latitude, the line drawn from the pole through this point will cut the equator in the point showing the length of the day or night. Thus, on the first of March, in latitude $50^{\circ}$ north, the length of the day appears to be nearly 10 hours and $\frac{3}{4}$, whence the sun must rise about 37 minutes after six; but in latitude $85^{\circ}$ the sun never sets on that day. P. 433.

\section{P L A T E XXXVI.}

Fig. 517. Projection of the constellations of the northern bemisphere on the plane of the equator. P. 433.

\section{P LA T E XXXVII.}

Fig. 518. Projection of the southern hemisphere. P. 433.

\section{PLATE XXXVIII.}

Fig. 519. A scale of the height of different parts of the earth's surface above the level of the sea, in English feet and miles, and in French toises. P. 439.

Fig. 520. A. The dotted ellipsis shows the section of a spheroid, which would be the form of the earth and sea if it were always in a state of equilibrium with the attraction of a distant body, and the shaded ellipsis the actual form assumed in consequence of its rotation round its centre, the depth of the sea being less than 13 miles. B. The surface of the sphere being supposed to be flattened, and the tides spread on it, they would assume the form of the waves here shown. The dotted straight line shows the mean height, which is a little above the surface in the principal sections of the spheroid, although not universally. C. The nature of the tides of lakes, the surface being regulated by that of the dotted line at $B$, nearly agreeing with it in direction, as at $D$, when the lake is narrow and deep, but differing from it, as at $\mathbf{E}$, when shallower. P. 444.

Fig. 521. The progress of the tides from the Atlantic through the channels surrounding the British islands, the lunar tides happening in any part of the shaded lines nearly at the hour after the moon's southing, which is indicated by the figure annexed to it. P. 446.

Fig. 522. The lines $A B$ and BC, representing the heights of the lunar and solar tides, and the angle A B C twice their angular distance, or A D C being simply the 
angular distance, the line $\mathrm{AC}$ shows the height of the compound tide, and the angles $B A C$ and A CB its distance from the lunar and solar tides respectively. P. 448.

Fig. 523. The two unequal tides represented by the elevation of the ellipsis above the smaller circle may be considered as composed of two equal tides cut off by the dotted circle, and the single tide between the two circles; as the tides $\mathrm{B}$ and $\mathrm{C}$ make the unequal tides at D. P. 449.

Fig. 524. The first and second curves represent two equal semidiurnal and one diurnal tide, which would make together two unequal tides: the third and fourth the same tides six hours more advanced : and when these are combined, the first and third destroy each other, but the second and fourth together compose the fifth, or a large diurnal tide. P. 449.

Fig. 525. A, the ancient system of the world, adopted by Ptolemy. B, the arrangement supposed by some other astronomers. P. 452.

Fig. 526. The Egyptian system of the world. P. 452.

Fig. 527. The system of the Pythagoreans, and of Copernicus. P. 454.

Fig. 528. The mode of representing the inequalities of the celestial motions employed by Ptolemy, the small circle being carried round the circumference of the larger, while the luminary revolves in it, so as to describe the dotted curve. P. 456 .

Fig. 529. The Tychonic system of the world. P. 457.

\section{PLATE XXXIX.}

Fig. 530. The repulsive force of two particles of matter, situated at the distance $\mathrm{AB}$ or $\mathbf{A ~ C}$, is represented by the ordinates or perpendiculars B D, C E, drawn to the curve D E, supposing the force to be inversely as the distance; but the law of the force appears to be more nearly represented by a curve like F E. The line DFG shows the magnitude of the cohesive force, which overcomes the repulsion at the distance A G, and is balanced by it when the particles arrive at the distance $\mathbf{A ~ B ~ o r ~ A ~ H . ~}$ The dotted lines represent the nature of the changes made in the lines FE, DFG, and FH, by an elevation of temperature. P. 474 .

Fig. 531. The general direction of the cohesive force acting on a particle of a liquid at $\mathbf{A}$ being represented by $\mathbf{A B}$ or $\mathbf{A C}$, that of the repulsive force will be $\mathbf{D A}$ or $\mathbf{E A}$, and in order to maintain the equilibrium, the forces $\mathrm{BF}$ and $\mathrm{CG}$, making together $\mathrm{HA}$, must be supplied by the pressure or reaction of the internal parts. P. 475 .

Fig. 532. A. The transverse section of a drop, supposed to be of considerable length, and flat at the sides : the curvature of the outline being every where proportional to its distance from the horizontal line A B. B, a round drop, the concavity at the horizontal line being equal to the convexity which would be found by cutting off the drop horizontally; the sum or difference of the curvatures being every where proportional to the distance from this line. P. 476.

Fig. 533. The solid A B possessing half the attractive power of the liquid CD, the surface of the liquid will remain horizontal : for the attractions will be represented by DA, DE, and DC; and of these DA and DE make DB, and DB and $\mathrm{DC}$ make DF, which is in a vertical direction. If the solid be more attractive, the forces will be combined nearly as at G, and if less attractive, as at H. P. 476 .

Fig. 534. The form of the surface of a liquid in contact with a plane and vertical side of a solid which is wetted by it. The height of the ascent of water is about one fourth of that which is here represented. P.477.

Fig. 535. The form of the surface of a liquid elevated between two plates which meet at $\mathbf{A}$, and are at a little distance from each other at $\mathbf{B}$; about one third of an inch, supposing the liquid to be water. P. 477.

Fig. 536. The beight at which water will stand in tubes of the form and magnitude which are here represented. P. 477.

Fig. 537. The depression of mercury, in contact with a large or flat glass vessel, is one fourth as great as that which is here represented. P. 478 .

Fig. 538. The depression of mercury within a small tube of glass. P. 478. 
Fig. 539. The actual elevation of a portion of water in contact with a horizontal surface which is wetted by it. P. 478.

Fig. 540. The elevation of mercury in contact with a horizontal surface of glass. P. 478.

Fig. 541. A, a wide drop of water standing on a dry surface, not attracting it. $B$, a wide drop of mercury, standing on glass. P. 478.

Fig. 542. A magnified representation of the manner in which the seeds of licopodium prevent a drop of water from wetting the substance on which it stands. P. 478.

Fig. 543. The bodies $A$ and $B$, and the bodies $C$ and $D$, appear to attract, and $\mathbf{E}$ and $F$ to repel each other. P. 479.

Fig. 544. The apparent cohesion of two plates, between which a fluid is interposed. P. 479.

Fig. 545. The apparent attraction of a drop between two plates, tending to draw it towards the line of their junction, causes the drop to rest in an inclined position of the plates. P. 479 .

Fig. 546. Dr. Herschel's figure, representing by the distance of the curve A BC from the line AC the heat thrown on different parts of $\mathrm{AC}$ by a prism, while DC is the illuminated part, divided according to Newton's experiments, the quantity of light being expressed by the distance of the line DEC. P. 490.

Fig. 547. Dr. Herschel's figure of the distribution of heat and light corrected according to the division of the coloured spectrum, as ascertained by Dr. Wollaston. P. 490.

Fig. 548. Bernoulli's air thermometer. P. 499.

Fig. 549. A differential air thermometer, or thermoscope, from which the pressure of the atmosphere is excluded. From Kunze. P. 499.

Fig. 550. A differential thermometer on Mr. Leslie's construction. P. 499.

Fig. 551. The distribution of the electric fluid in spheres of different sizes, and at different distances, and in a conical point. The density is represented by the distance of the dotted line from the surface. P. 511.

\section{P L A T E XL.}

Fig. 552. A. A spark passing between a negative and a neutral ball; B, between a neutral and a positive ball; $C$, between a negative and a positive ball. $D$, two sparks between a negative and a positive cylinder, each of the same form as if it were passing singly from the end of a charged to the side of a neutral cylinder. From Mr. Nicholson. P. 518.

Fig. 553. A compound galvanic circuit, formed by portions of an acid, pieces of zinc, and wires of silver; the arrows show the directions of the electric current. P. 522.

Fig. 554. A compound galvanic circuit, formed by an acid, charcoal and water, the water and acid communicating by a small siphon. P.522.

Fig. 555. A compound galvanic circuit, formed by portions of an alkaline sulfuret, and water, and pieces of copper: the liquids being connected by a siphon. Fig. 522.

Fig. 556. A simple galvanic circuit, formed by wires of zinc and silver, or platina, the lower ends being immersed in an acid, and the upper being brought into contact at pleasure. P. 522.

Fig. 557. A galvanic battery, in the form of a trough, composed of plates of zinc, silvered on one side, with vacant spaces for the reception of an acid: the letters show the order of the elements, and the arrows the direction of the current, from the positive wire + to the negative wire - P. 523 .

Fig. 558. An electrical machine, on Nairne's construction. A, the cylinder of glass ; B, the cushion, or rubber ; C, the silk flap; D, the negative conductor; $E$, the positive conductor; F, a ball connected with the internal coating of a glass jar, contained in the conductor. The conductors are insulated by varnished rods of glass. P. 525 . 
Fig. 559. A plate machine. A and B, the rubbers, which are usually double; C D, double flaps of oiled silk, for confining the electricity; E, the conductor. P. 525 .

Fig. 560. An electrophorus. A, the cake of resin; B, the plate of metal; C, the ball for taking the spark; D, the handle of glass. P. 526.

Fig. 561. A condenser, as arranged by Mr. Cavallo, under the name of a collector : the middle plate is insulated : the two outward plates communicate with the earth; they stand near the first plate when the electricity is imparted to it, and are afterwards removed by means of their hinges. P. 526 .

Fig. 562. Mr. Cavallo's multiplier. The electricity being first communicated to the insulated plate $\mathbf{A}$, the moveable plate $\mathrm{B}$ is brought near it, while the wire $\mathbf{C}$ touches the pin $D$ so as to form a communication with the earth; the plate $B$ is then made to communicate with $\mathbf{E}$, which is insulated, and stands near the plate $\mathbf{F}$, which enables it to receive almost the whole of the electricity brought at each alternation by $\mathrm{B}$; and when the plate $\mathrm{F}$ is removed from the neighbourhood of $\mathrm{E}$, this plate becomes strongly charged. P. 527 .

Fig. 563. A revolving doubler, on the principle of Mr. Bennet's instrument. The fixed and insulated plate $\mathrm{A}$ first receives the electricity, and when the moveable plate B stands opposite to it, it receives by a wire from the stand of the instrument C the opposite electricity; when it is brought opposite to $\mathrm{D}$, this plate is made to communicate with the stand by the wire $\mathbf{E}$, and acquires a charge similar. and nearly equal to that of $\mathbf{A}$. When $\mathbf{B}$ comes again to $\mathbf{A}$, the wire $\mathbf{F}$ forming a communication between $A$ and $D$, nearly the whole charge of both these plates is brought into $A$, and $B$ receives a charge almost twice as great as at first. ${ }^{\circ}$ P. 527.

Fig. 564. Mr. Coulomb's electrical balance. The needle $\mathrm{A}$ is made of silk, covered with sealing wax; it supports, at the end B, a ball of the pith of elder, another similar ball being fixed at $\mathbf{C}$; the force of attraction or repulsion is ascertained by the torsion of the wire $\mathbf{A ~ D}$, which is measured by a graduated circle $\mathbf{E}$. P. 528 .

Fig. 565. Mr. Henry's quadrant electrometer; it is made of box wood, supported by metal : the ball is of cork, the graduated arc of ivory. P. 528.

Fig. 566. A, Mr. Bennet's gold leaf electrometer; B, a piece of excited sealing wax held over it, for distinguishing the electricity. Instead of the pieces of gold leaf C, we may substitute Mr. Cavallo's pith balls D, or the straws E, employed by Volta. P. 528 .

Fig. 567. Mr. Lane's discharging electrometer. The distance of the balls A, B is measured by the turns of the screw on the scale $\mathbf{C}$; and the parts of a turn are ascertained by the graduated circle D. P. 528 .

Fig. 568. A discharger for a battery. When the repulsion of the balls A, B, becomes greater than the weight of a wire which passes through a perforation in the balls, they separate, and the ball $\mathbf{C}$, descending to $\mathrm{D}$, forms a communication, which completes the circuit, so that the shock passes through any substance placed at E. P. 528.

\section{P LATE XLI.}

Fig. 569. The form of the curves which show the direction of the magnetic needle, in consequence of the attraction and repulsion of two poles, situated at $\mathbf{A}$ and B. They are found by drawing the lines ACD,BED, so that the sum or difference of the parts A C, B E, shall be always equal, A CE B being a semicircle ; and the direction D F may be found by making A F to BF as the cube of A D to that of B D. P. 534 .

Fig. 570. The arrangement of iron filings in the neighbourhood of a magnet. P. 534.

Fig. 571. The particle of iron A B, lying on a card nearly over the magnet C, assumes, when the card is shaken, first the position $\mathbf{D}$, then, falling to $\mathbf{E}$ and $F$, is left a little further from the magnet than at first. P. 534 .

Fig. 572. An azimuth compass. The box is turned round, until the shadow of the thread A B or A C falls on the line CD: the position of the needle is then 
ascertained by that of the card $\mathbf{E}$, which is fixed on it. The compass is kept always in a horizontal position, by means of a double suspension on the gimbals $\mathbf{E} \mathbf{G}$. Instead of this suspension, Mr. $\mathbf{M}^{\prime}$ Culloch makes the bottom of the box in the form of a hollow cone, resting on a point, and loaded with a weight, which brings the centre of gravity below the point of support, as at H. P. 535 .

Fig. 573. A dipping needle. The piece A B is brought into such a situation, that the line drawn on it coincides with the middle of the vibrations of the needle. The position of the needle may be changed, either by turning the stand half round, or by turning the needle within the stand. P. 535 .

Fig. 574..576. The situations of the lines of equal declination in 1700, 1744, and 1794, in the hemisphere, which is bisected by the meridian of London. The first two from Mountaine's Tables, * the last from Churchman's Chart. $\dagger$ P. 536.

Fig. 577. The actual situations of the lines of equal dip. From Churchman's Chart. P. 537.

Fig. 578. The lines of equal dip, calculated from the supposition of a small magnet, situated at the centre of the earth, directed to a point in latitude $75^{\circ} \mathrm{N}$. and longitude $70^{\circ} \mathrm{W}$. P. 537 .

Fig. 579. A, Six's thermometer ; B, the wire with a fine spring, which serves as an index. P. 545 .

Fig. 580. Rutherford's double thermometer. P. 545.

Fig. 581. Deluc's whalebone hygrometer. A, the slip of whalebone; B, a spiral spring, serving to keep it stretched; C, the index. P.554.

\section{PLATES XLII. XLIII.}

Fig. 582. A chart of the world, on Mercator's projection, from Arrowsmith; with the dip and variation of the compass, principally from Churchman, for the year 1794 ; and with the trade winds and monsoons. P. 437, 536.

* Ph. Tr. 1757, p. 329. Account of Methods, 4to, 1758.

† Churchman's Magnetic Atlas, 1794. 


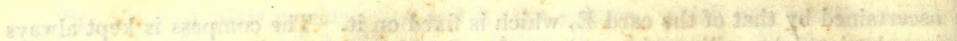

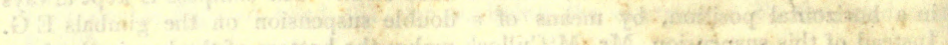

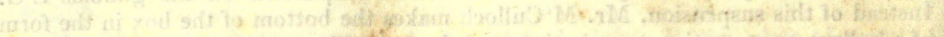

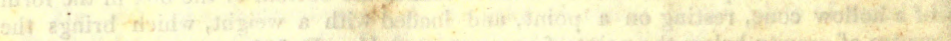

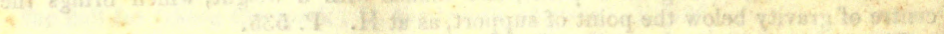

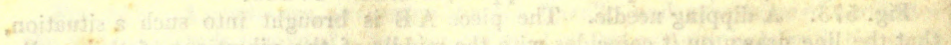

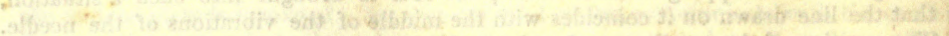

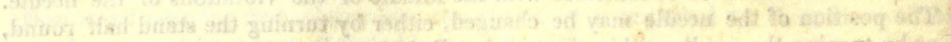

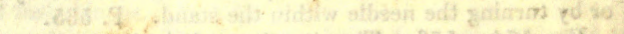

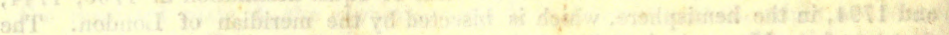

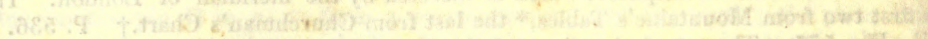

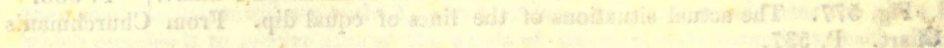

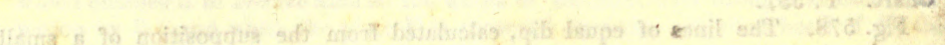

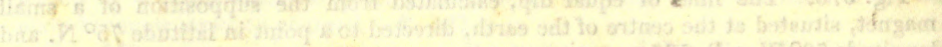

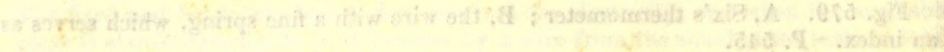

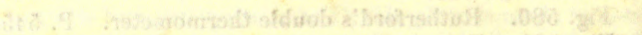

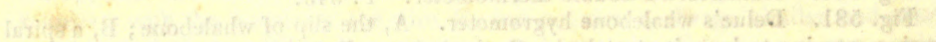

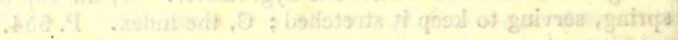

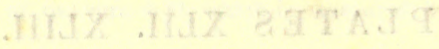

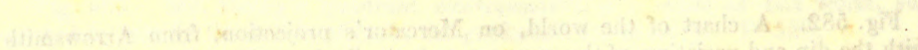

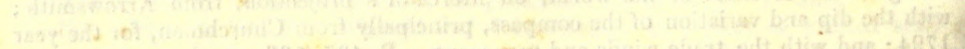

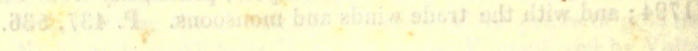

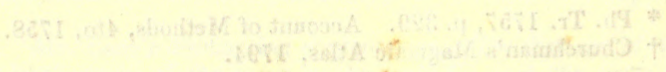




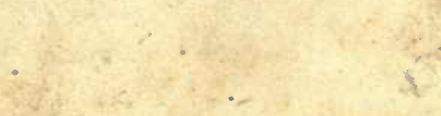

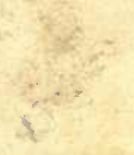

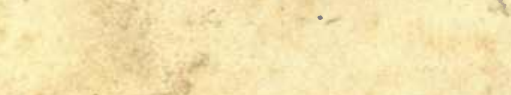

3.t.

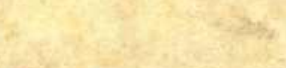

8

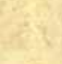



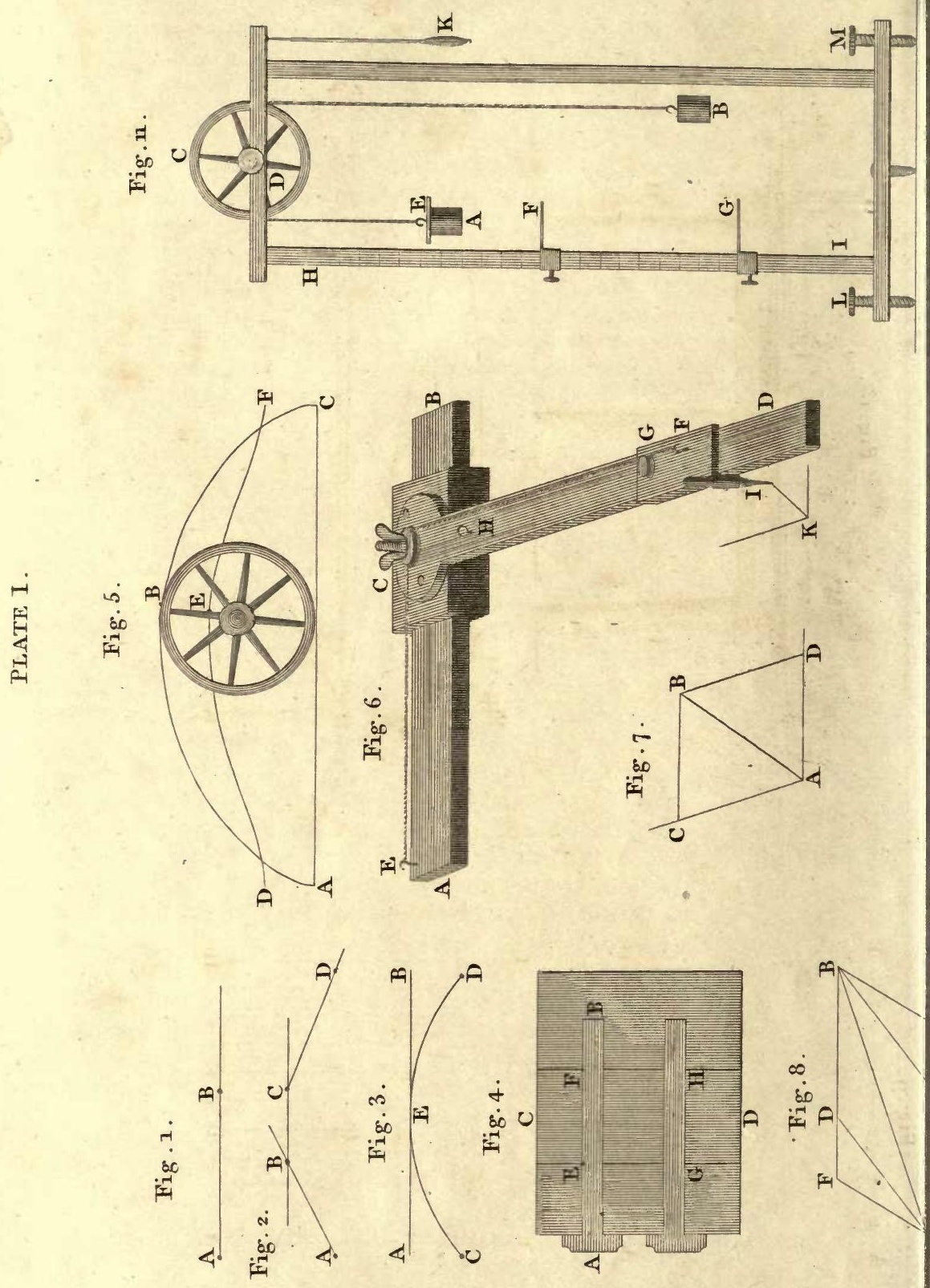

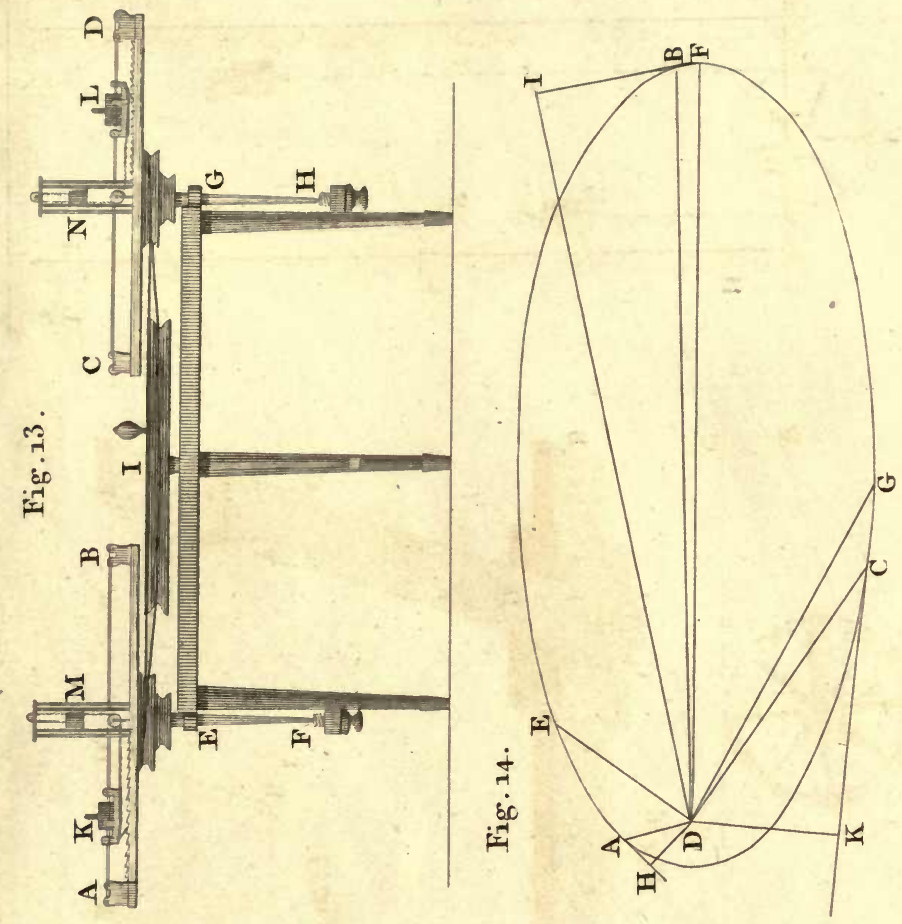

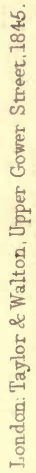
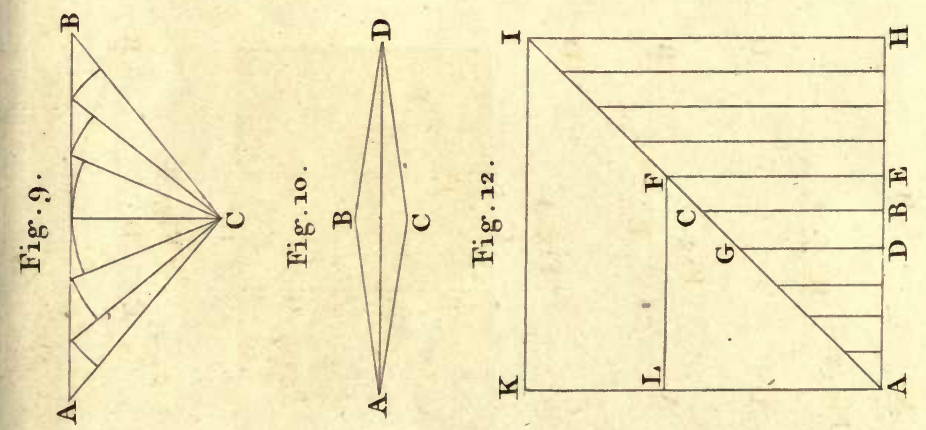



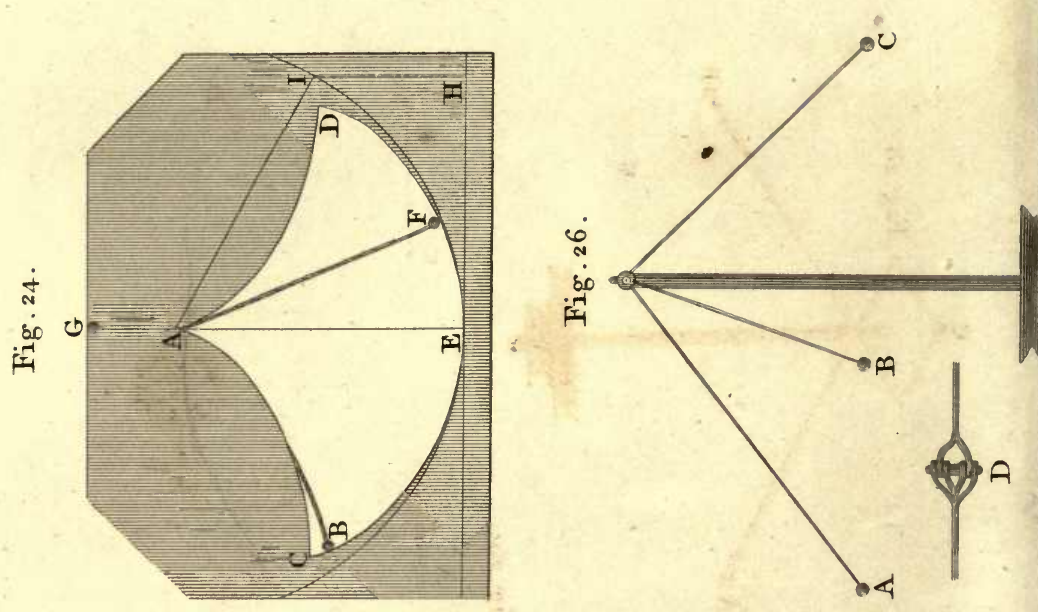

E
a-
$\frac{5}{4}$
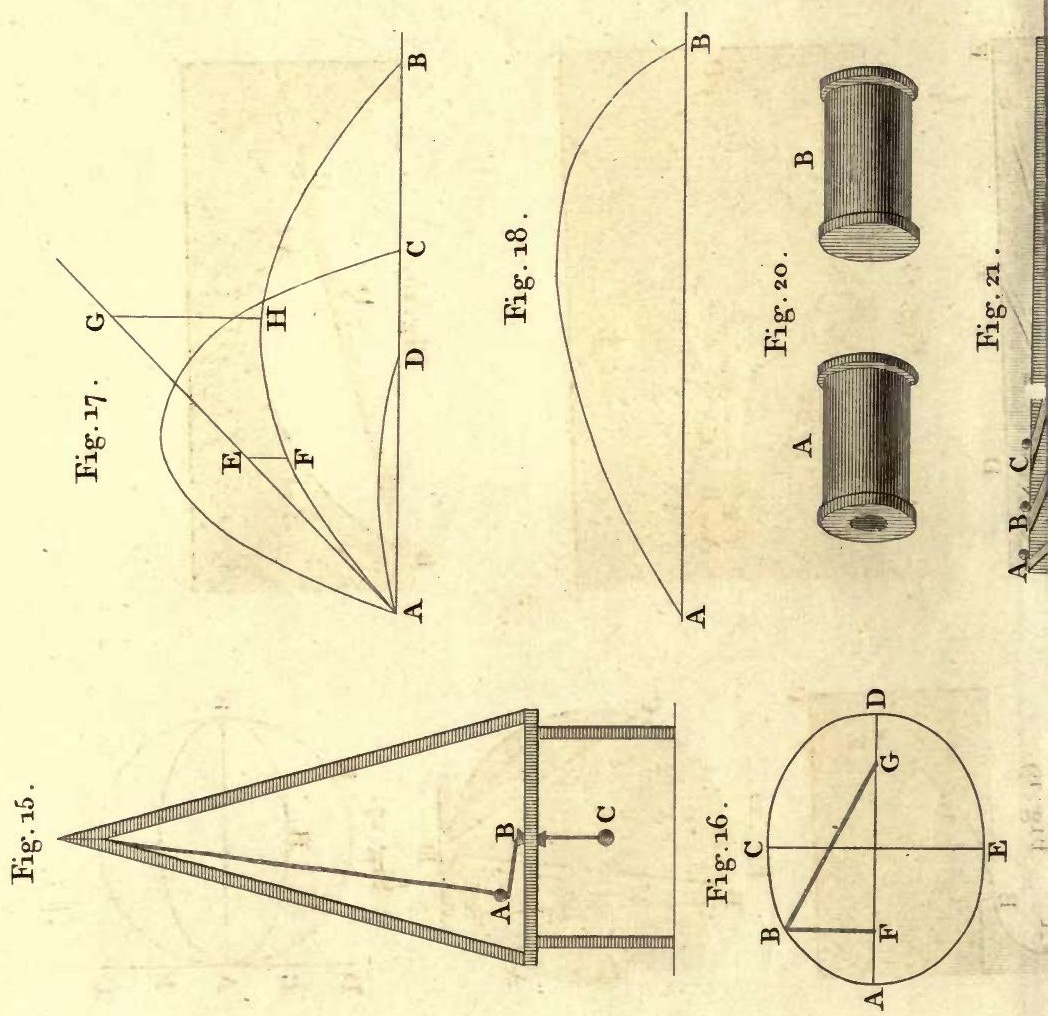

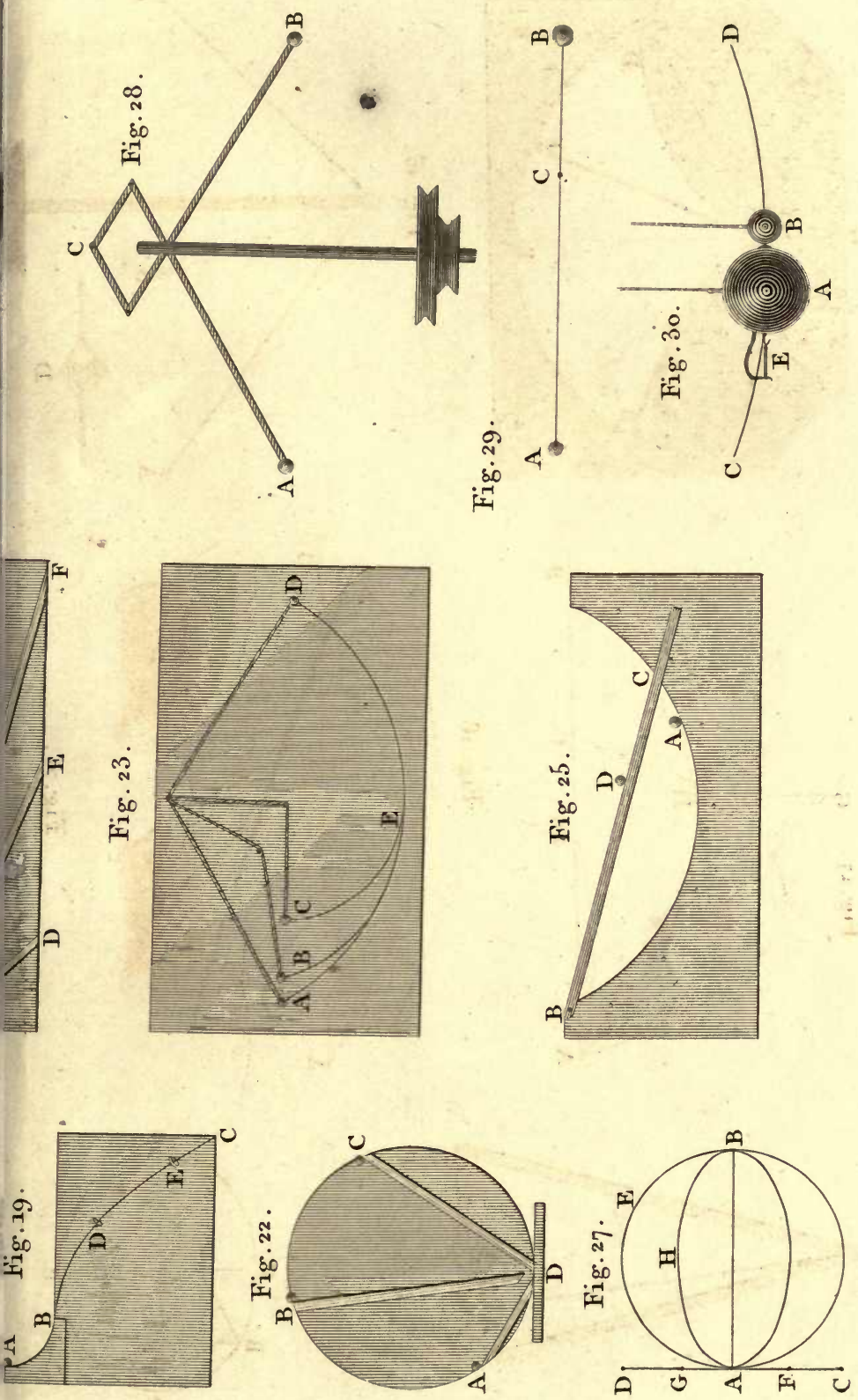



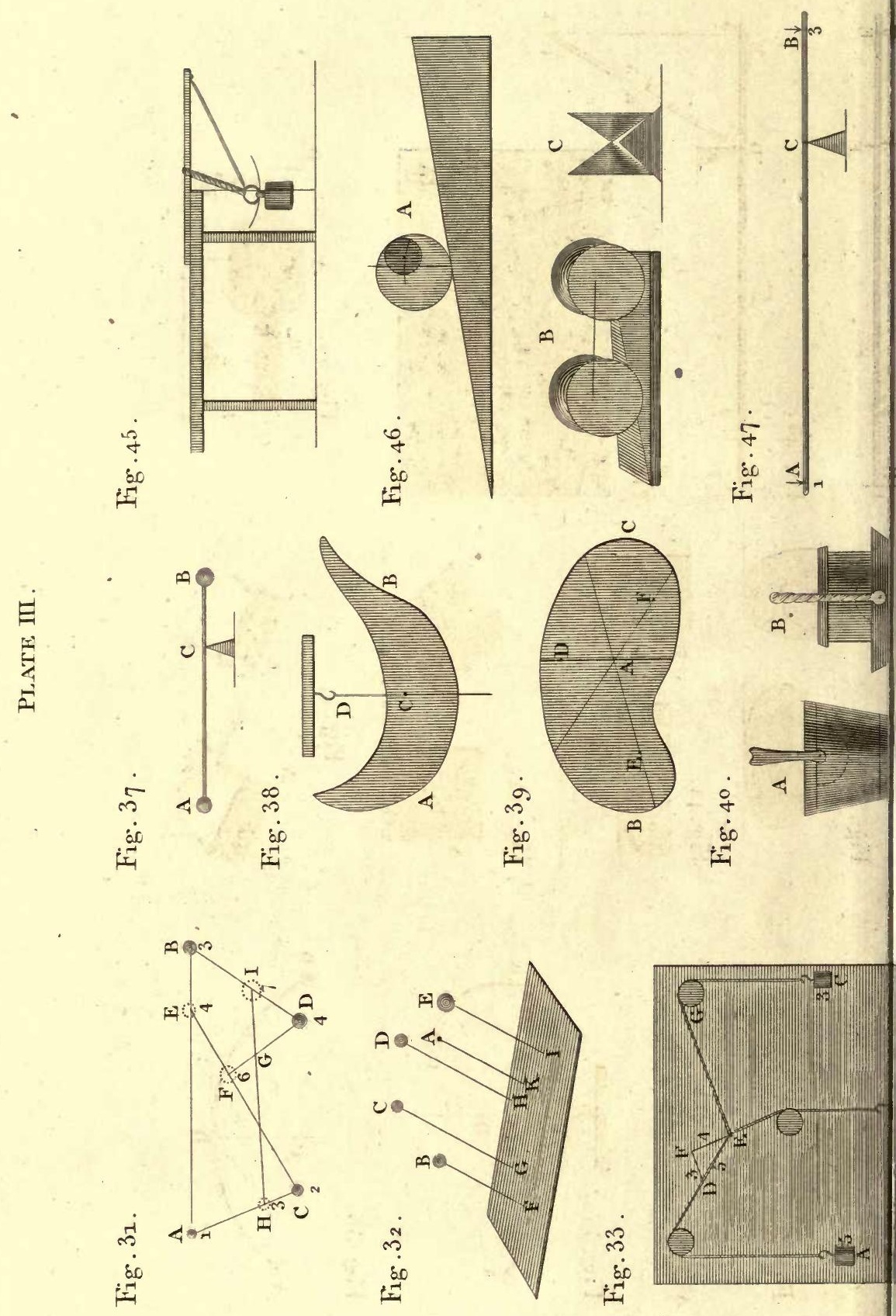



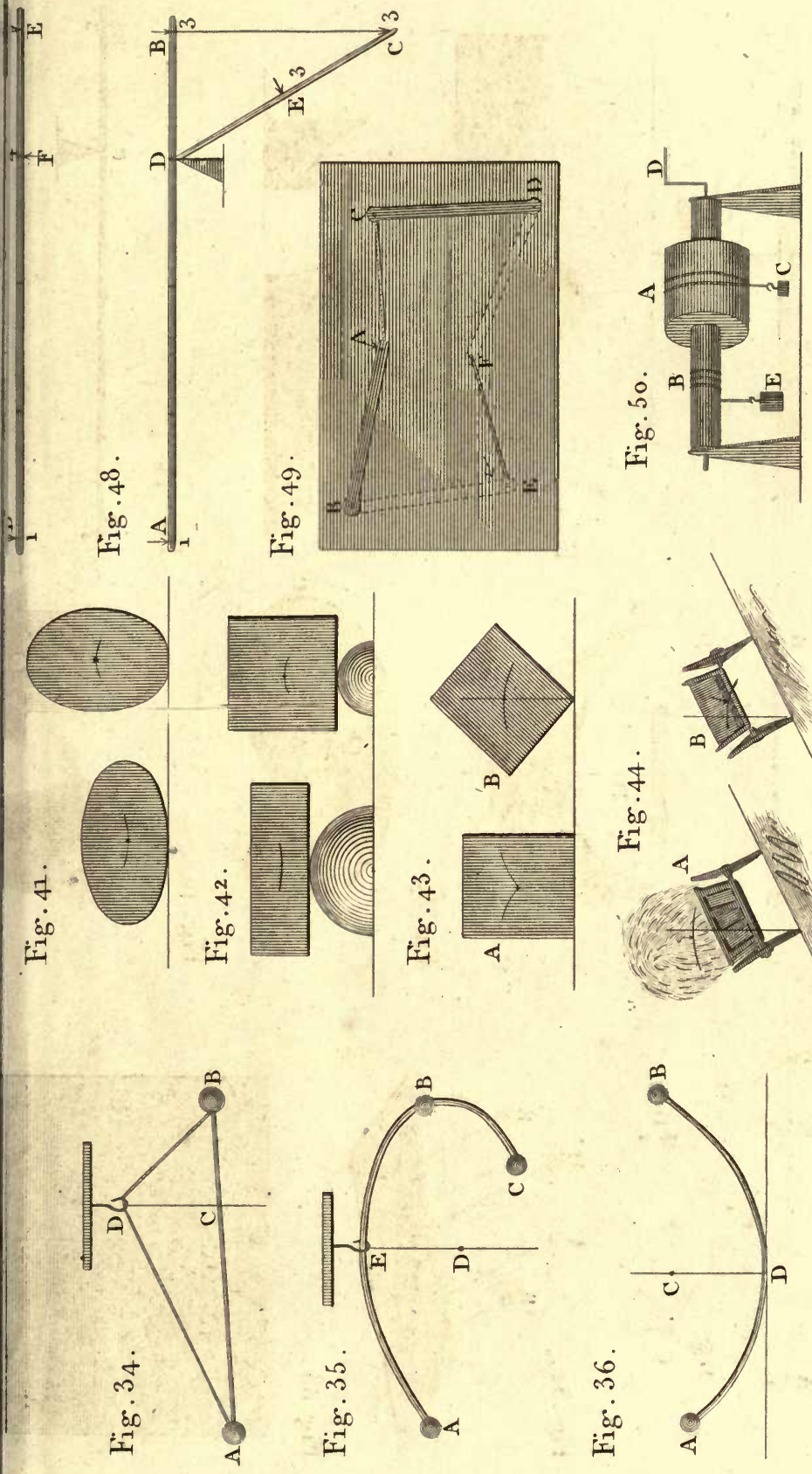



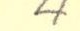



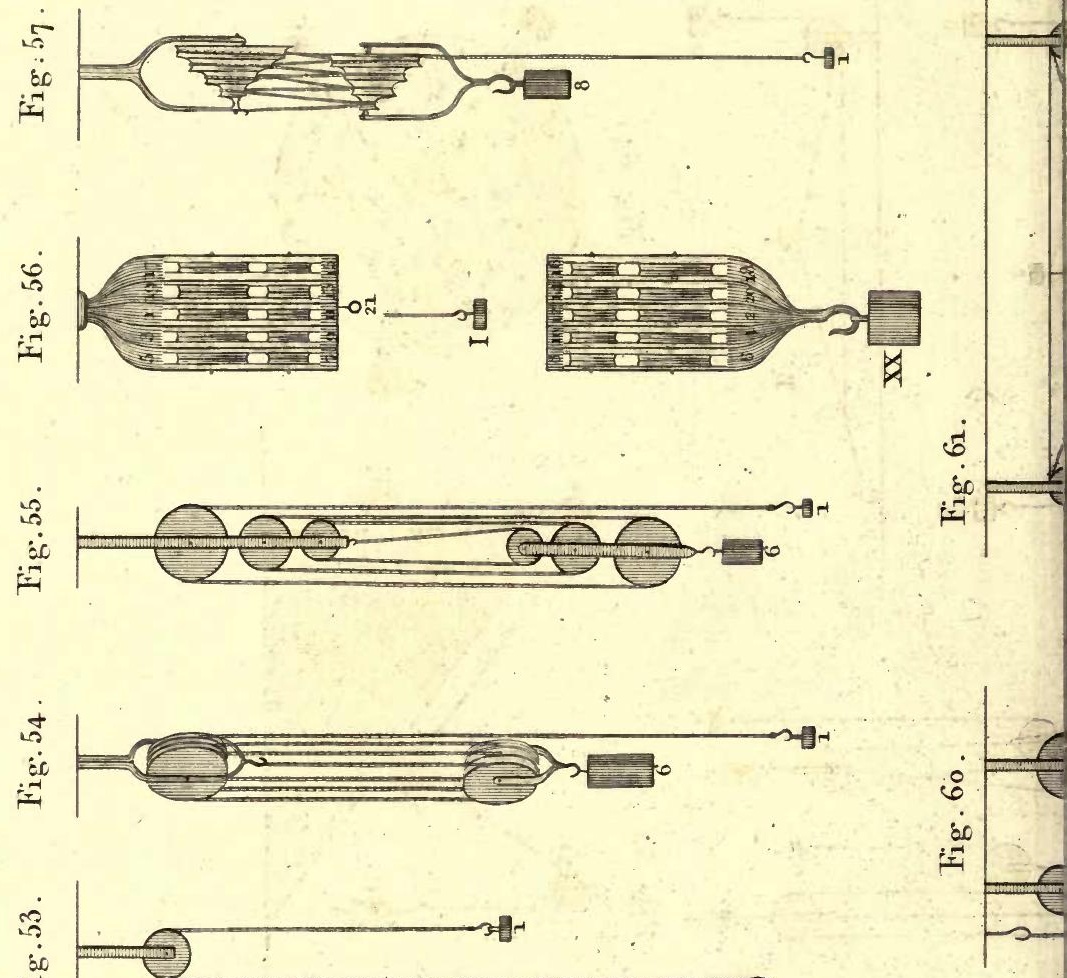

7
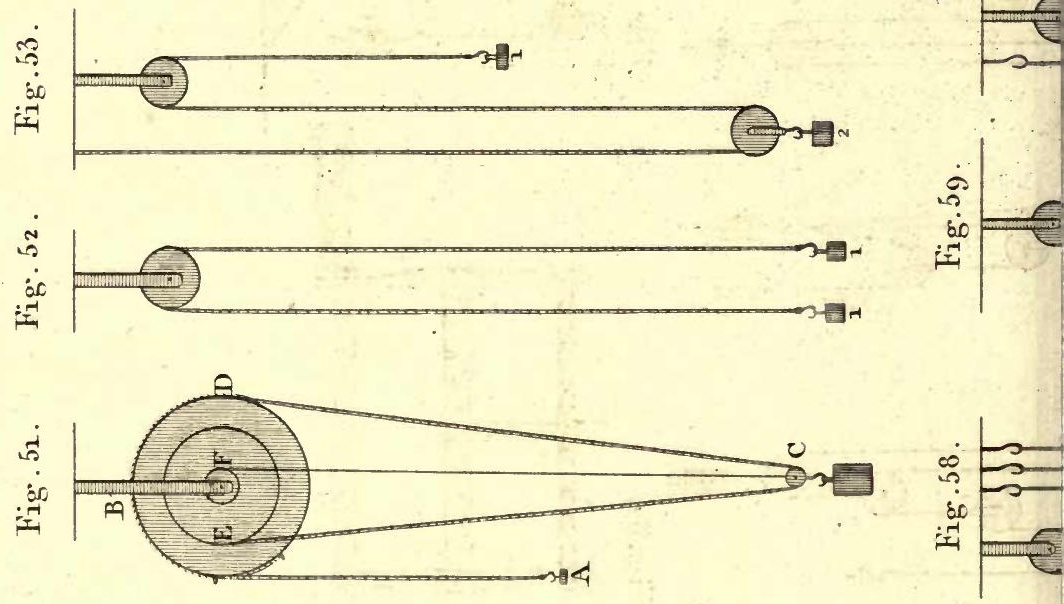


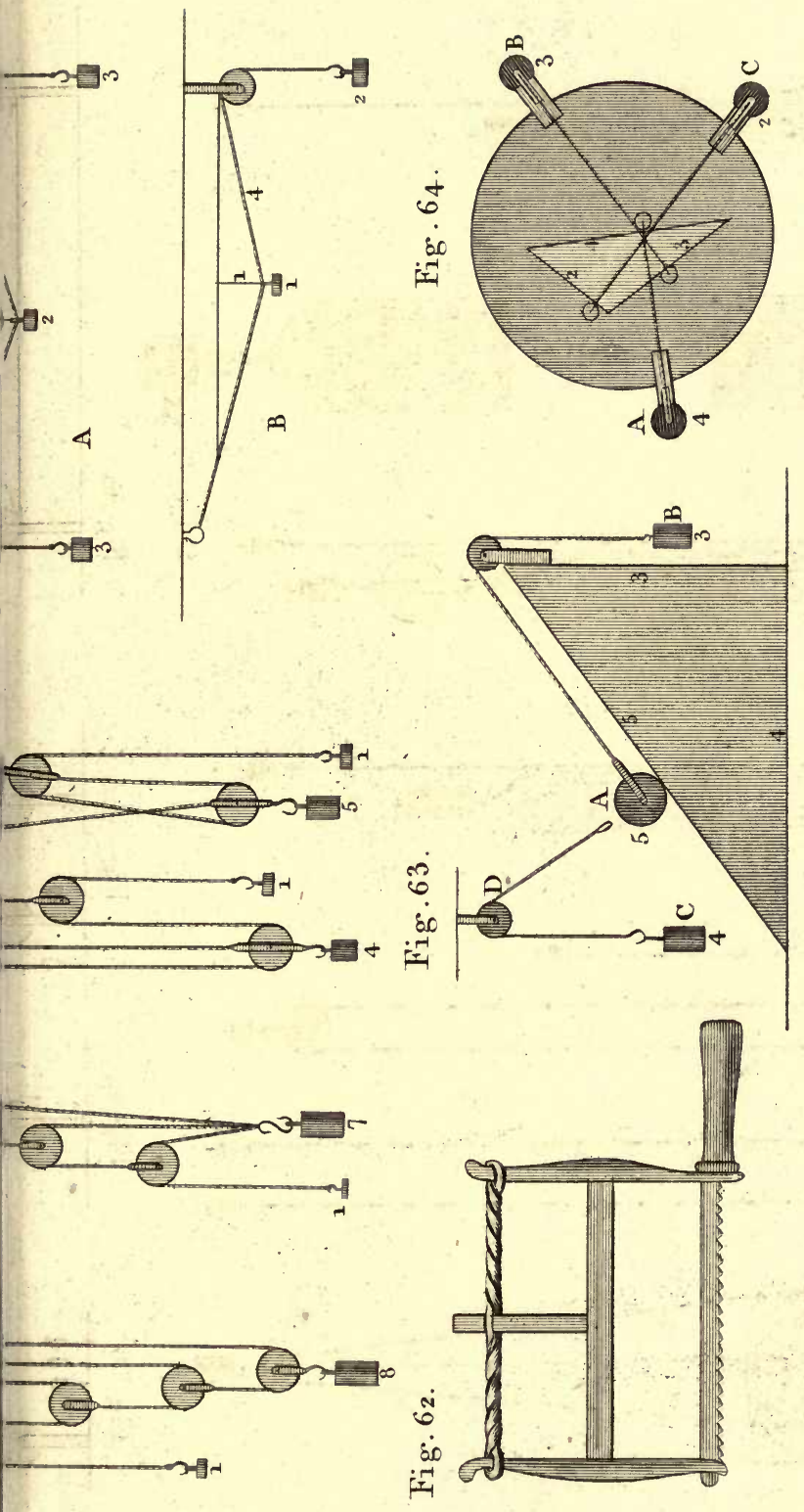



(2) से 


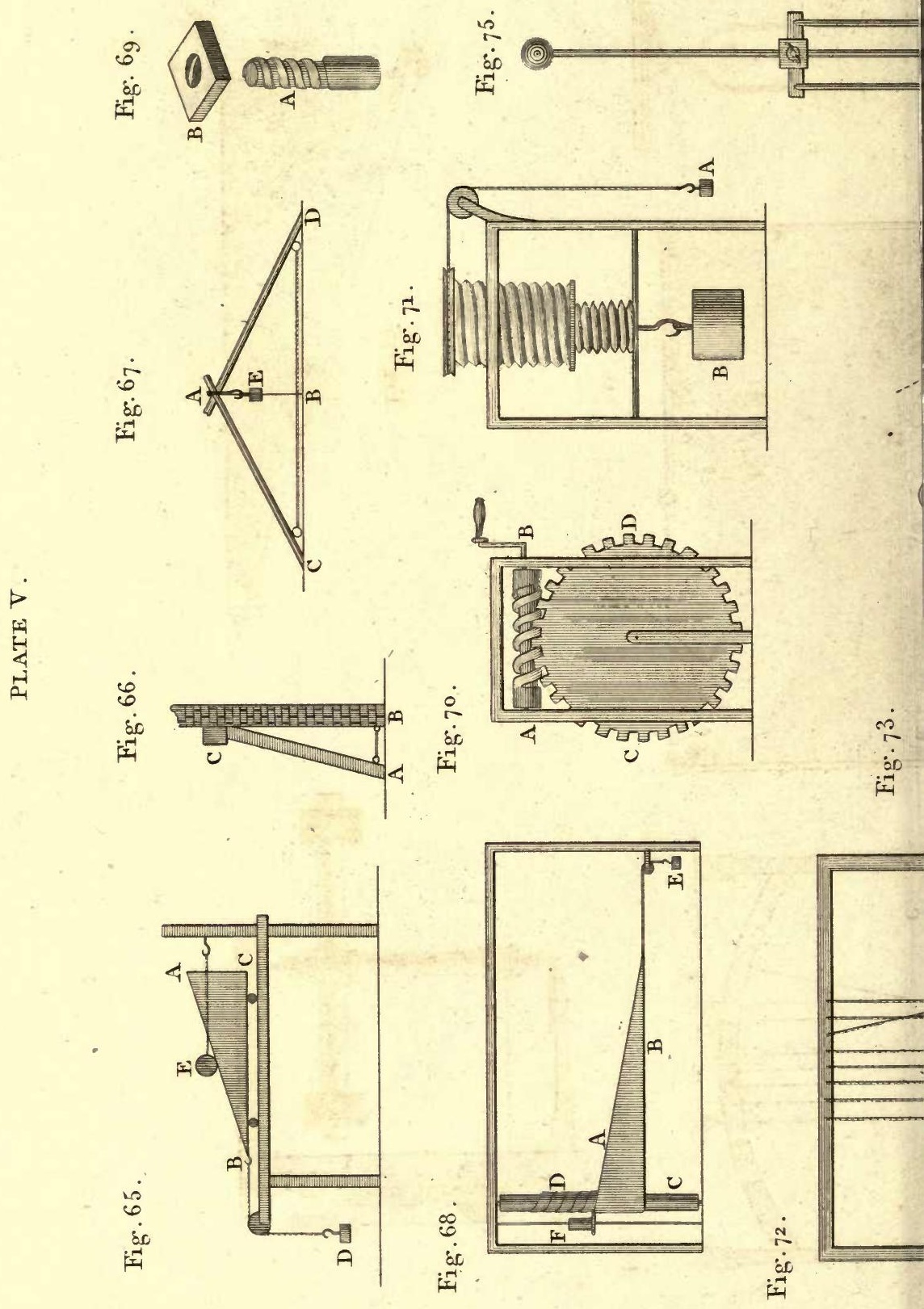




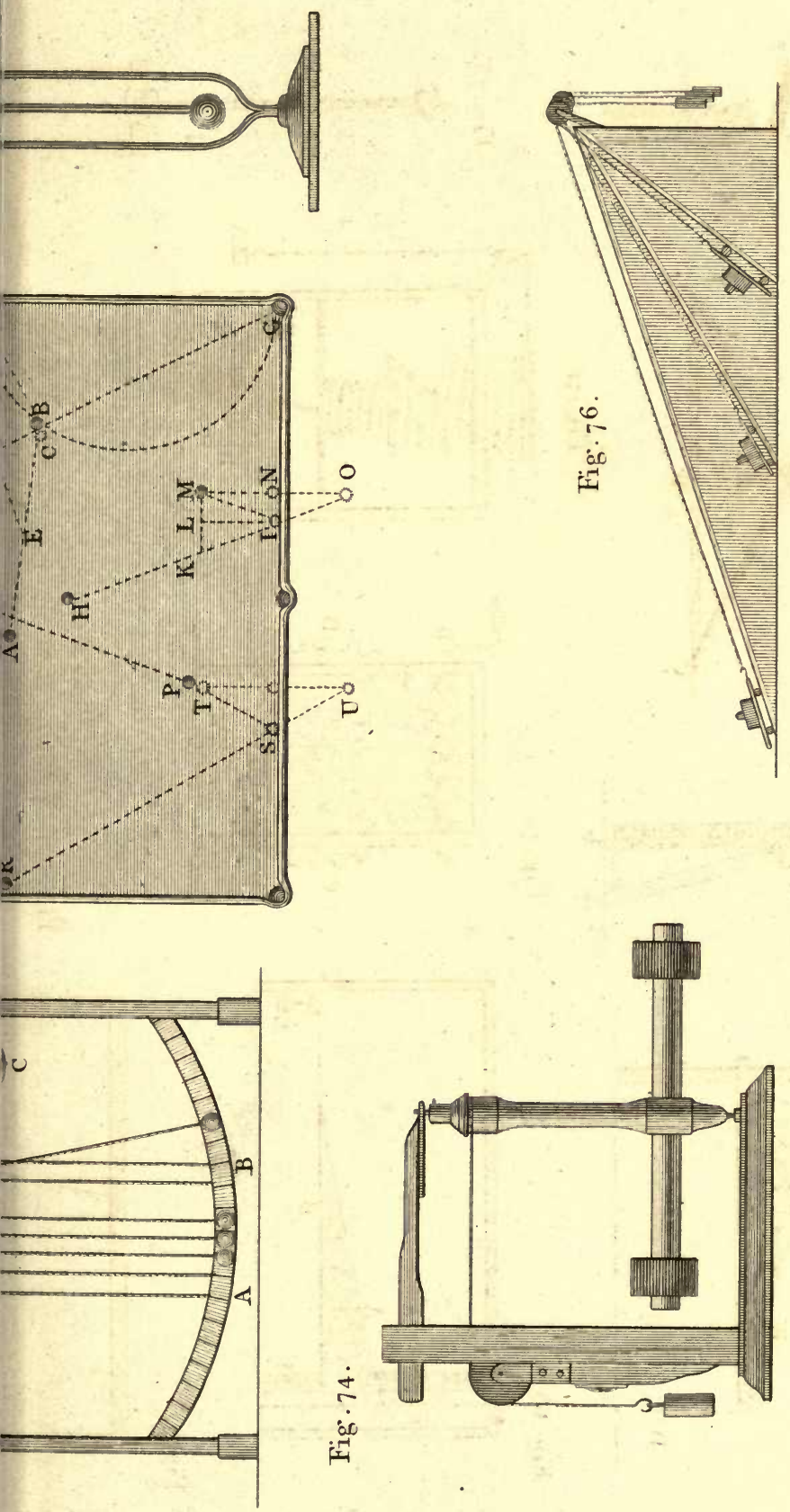

 


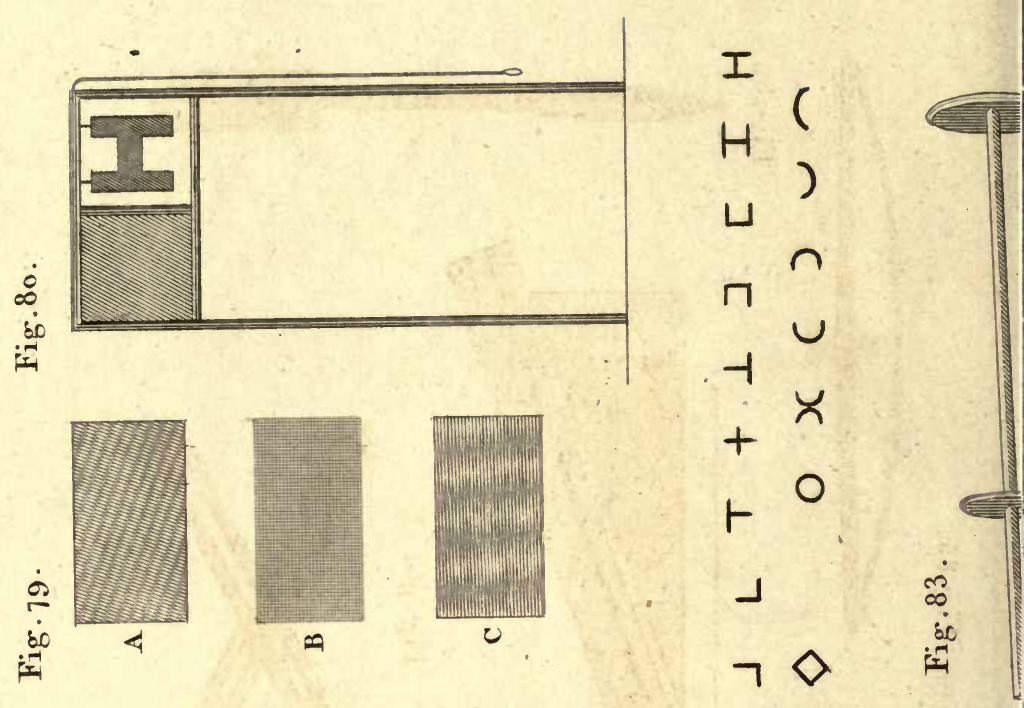

एं
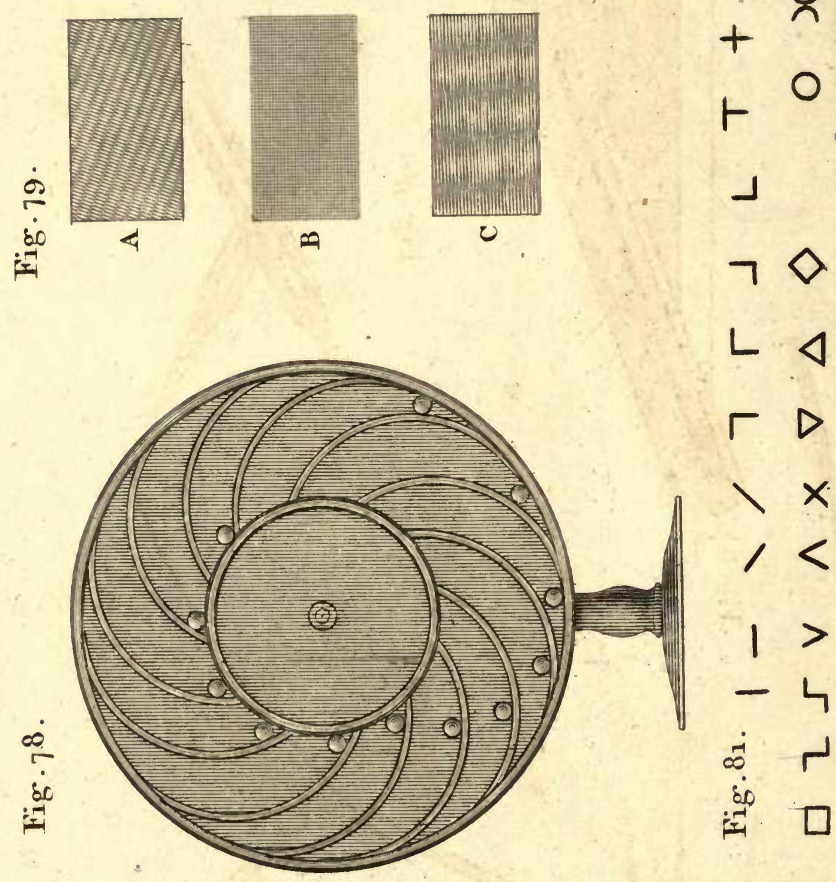

$\Gamma D$

$>x$

, <

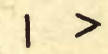

$-4$

-

这

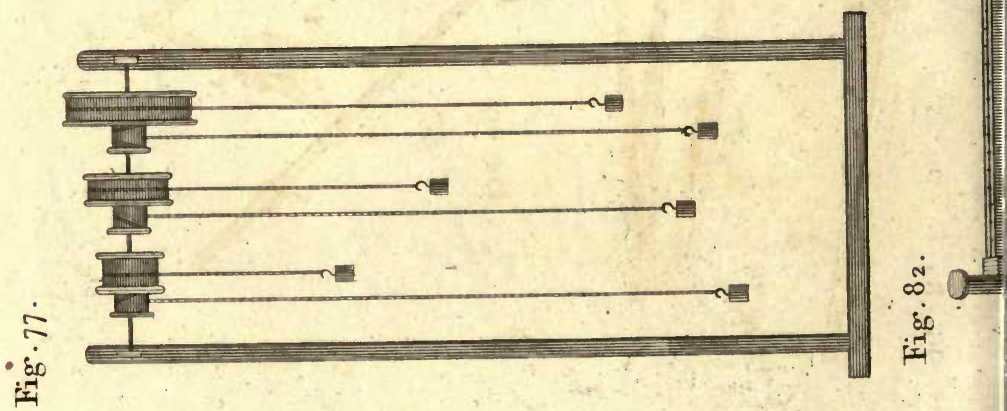




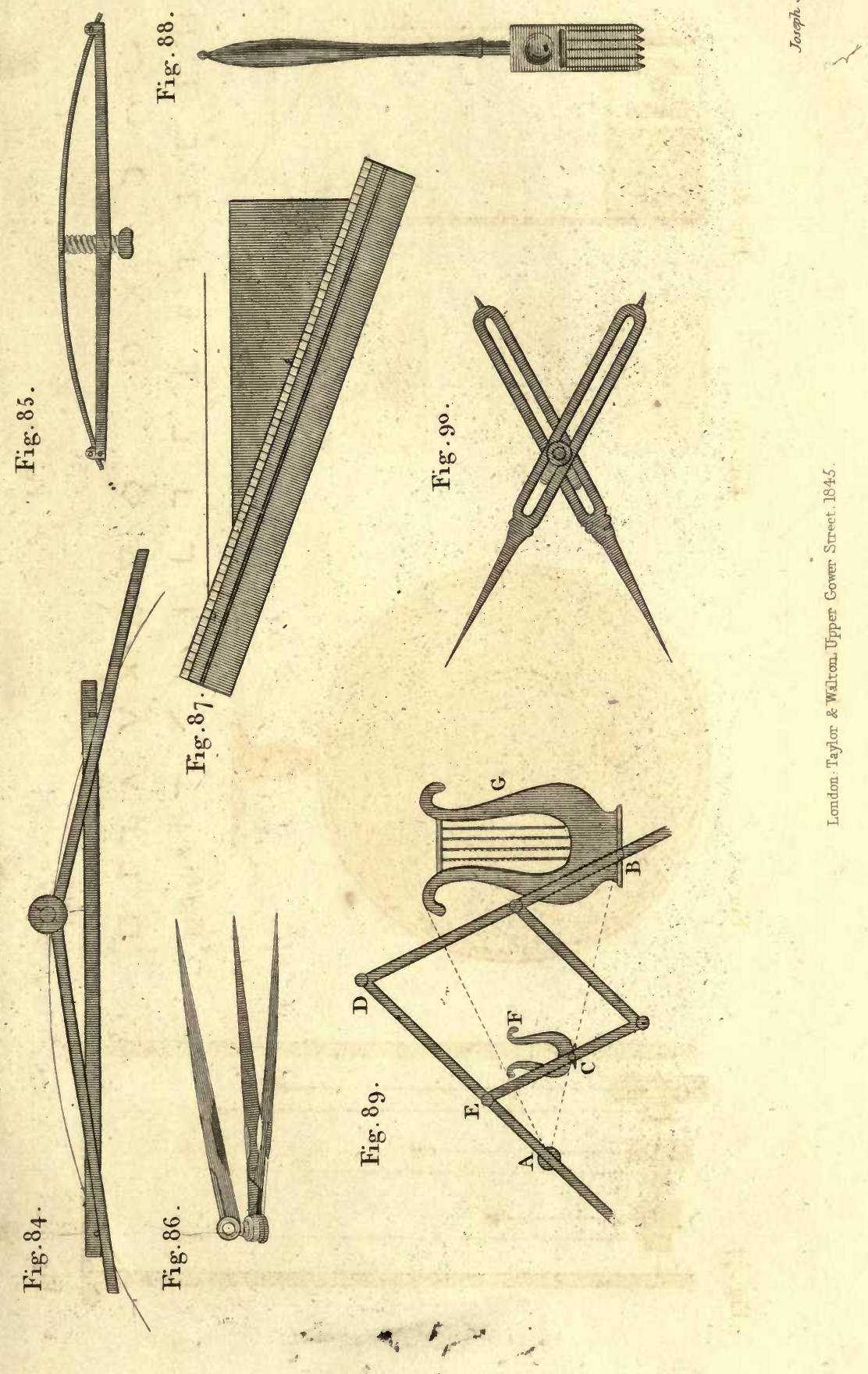




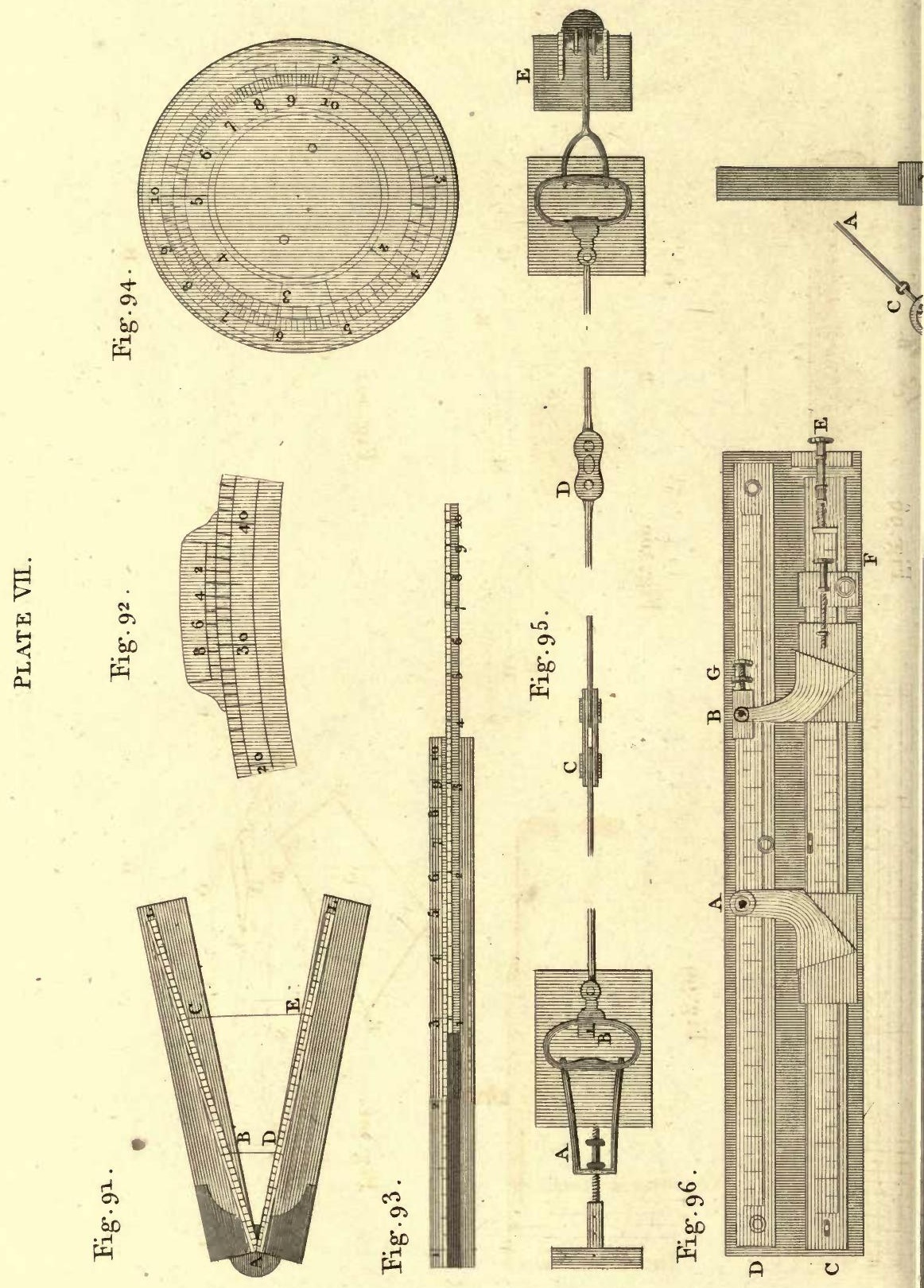


运

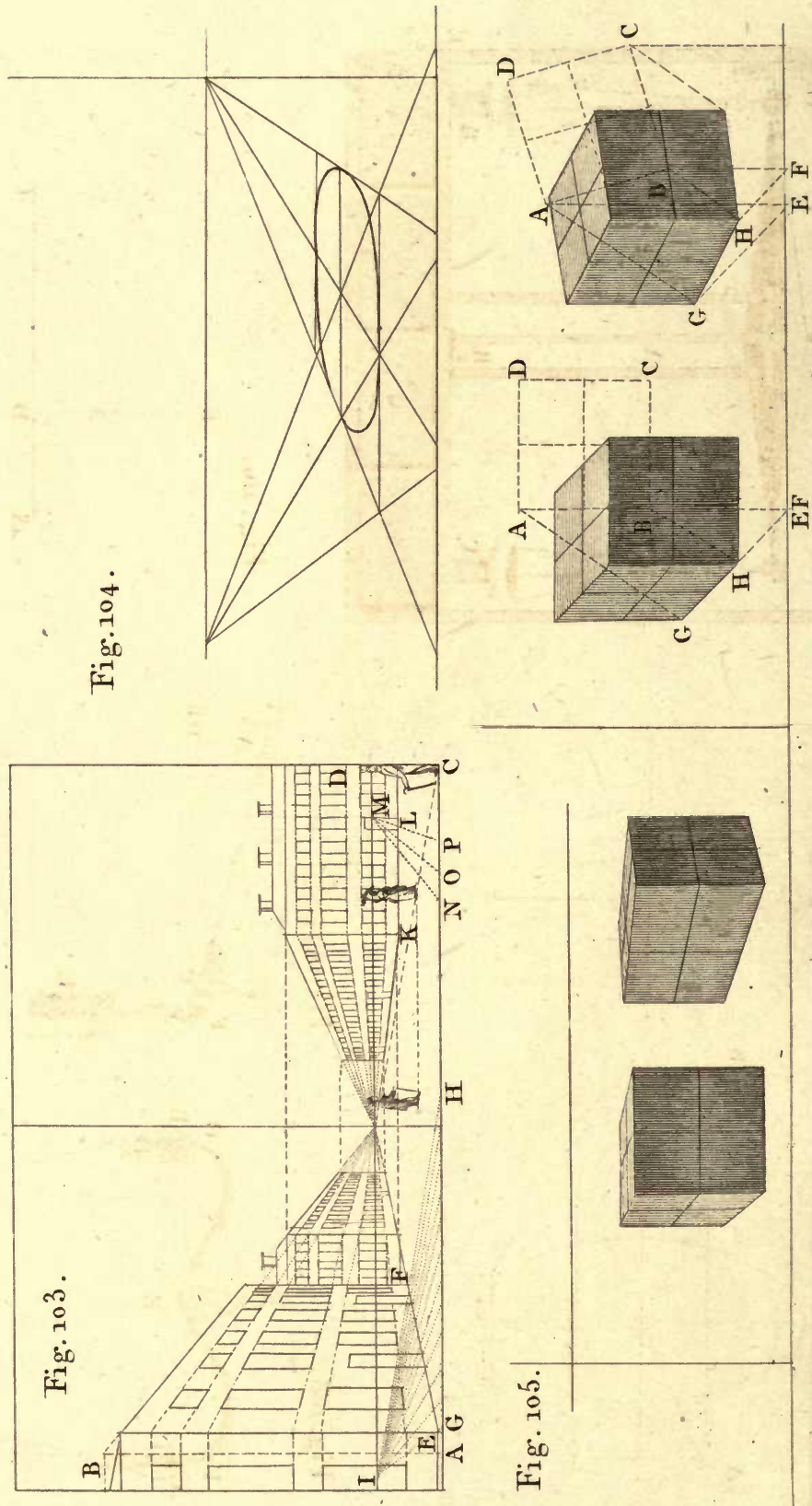





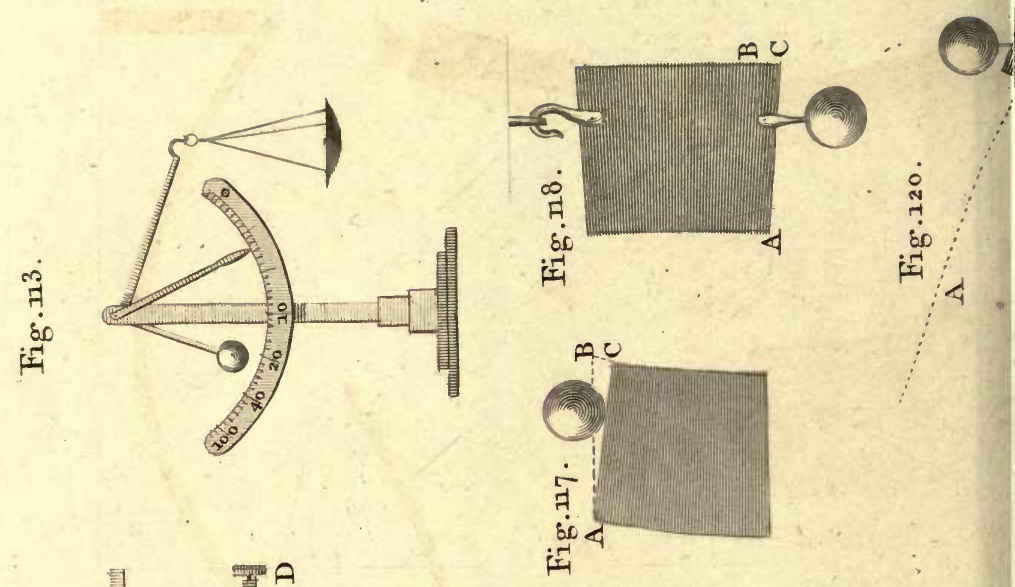

界
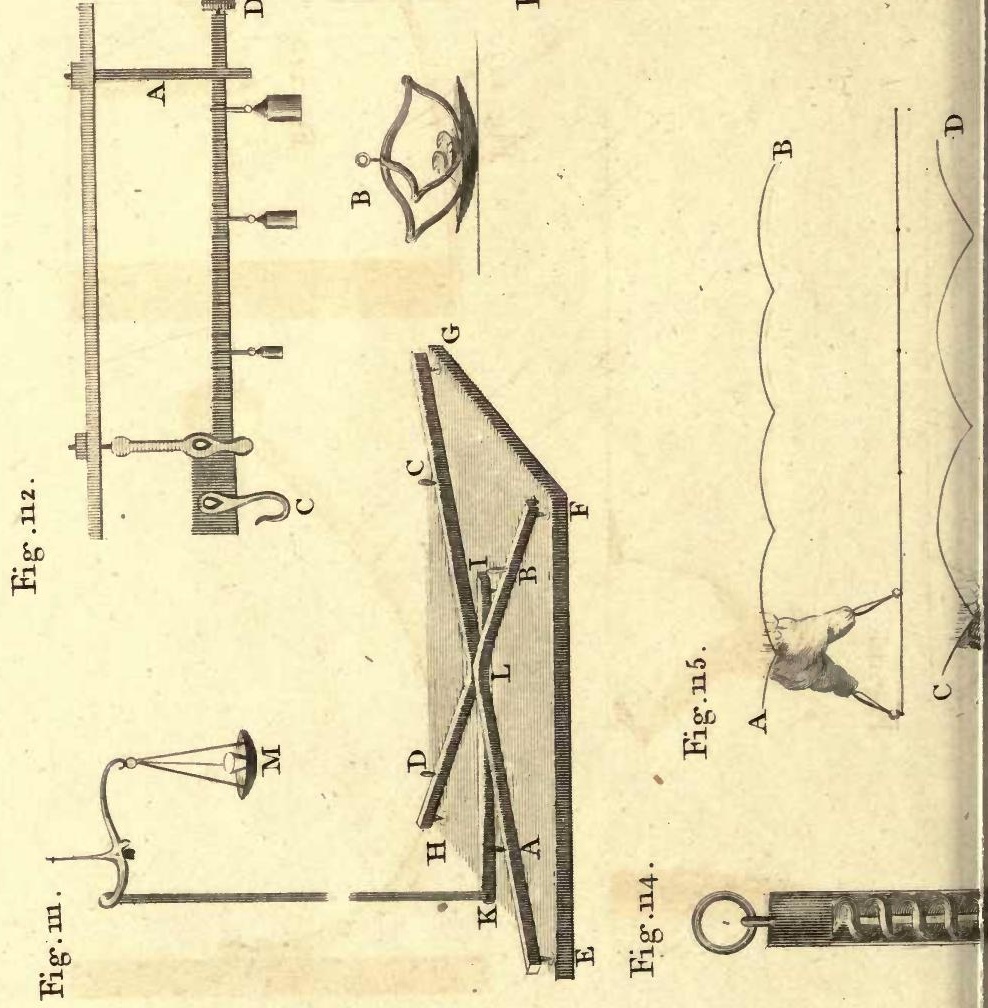


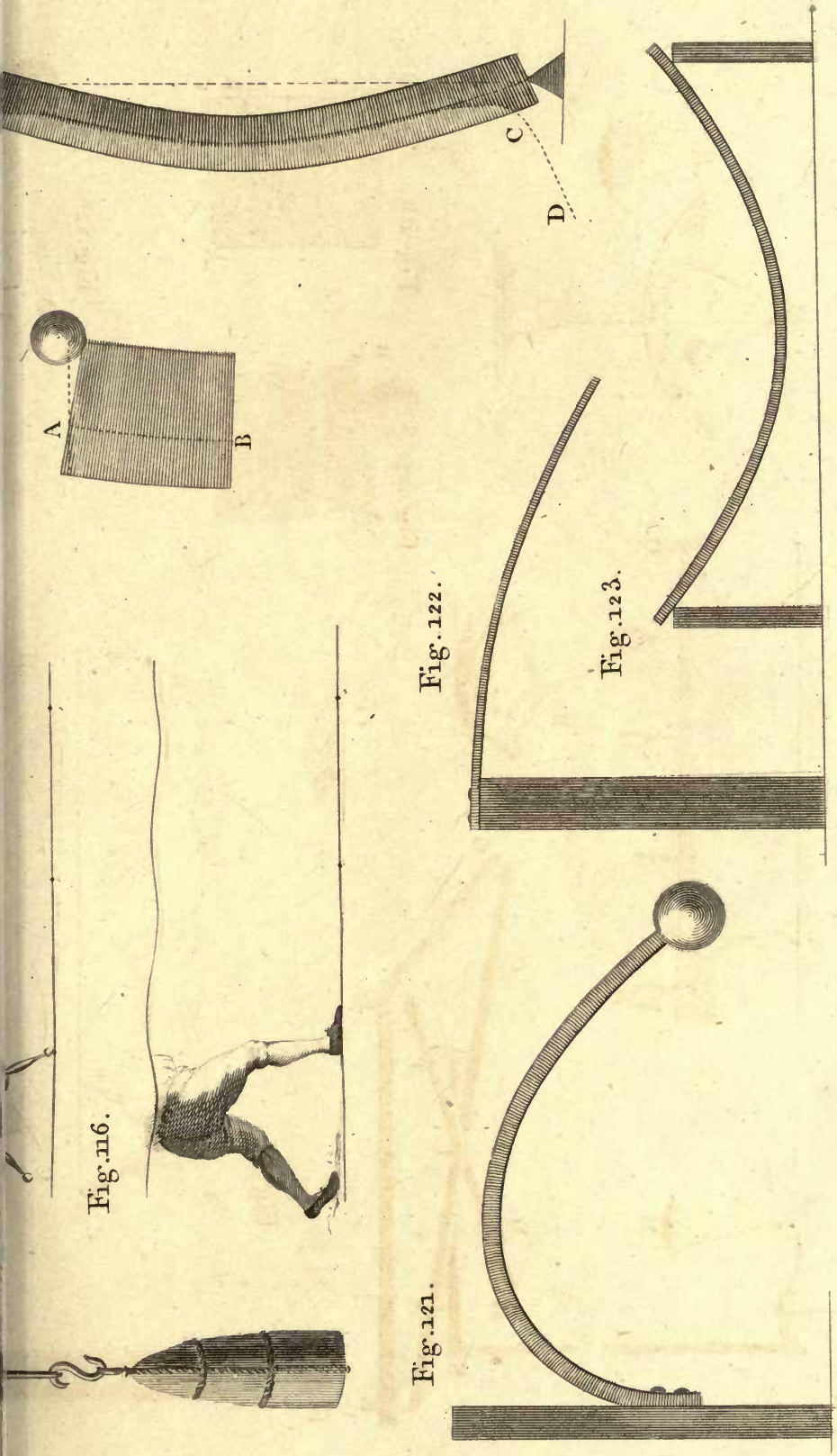




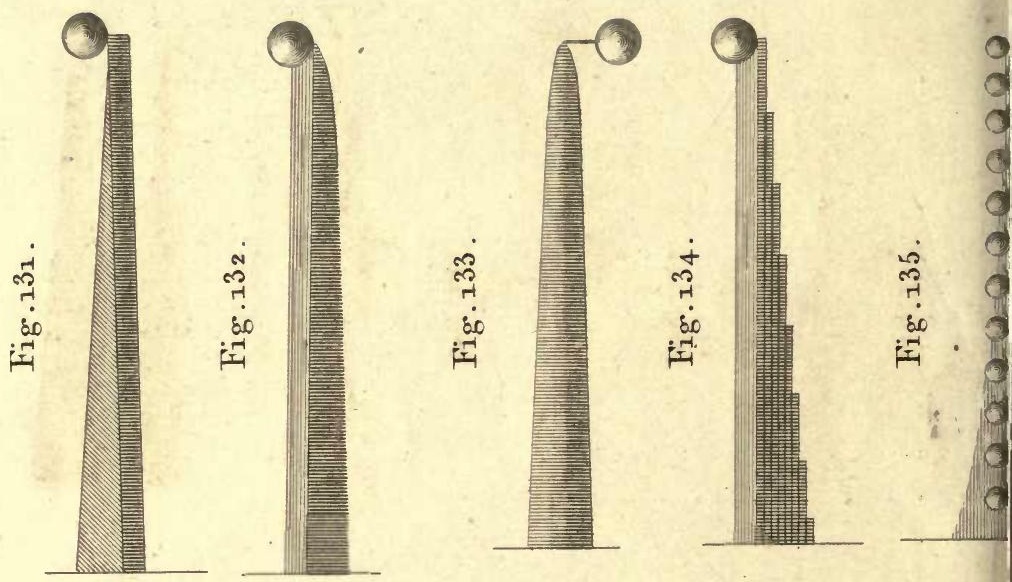

过
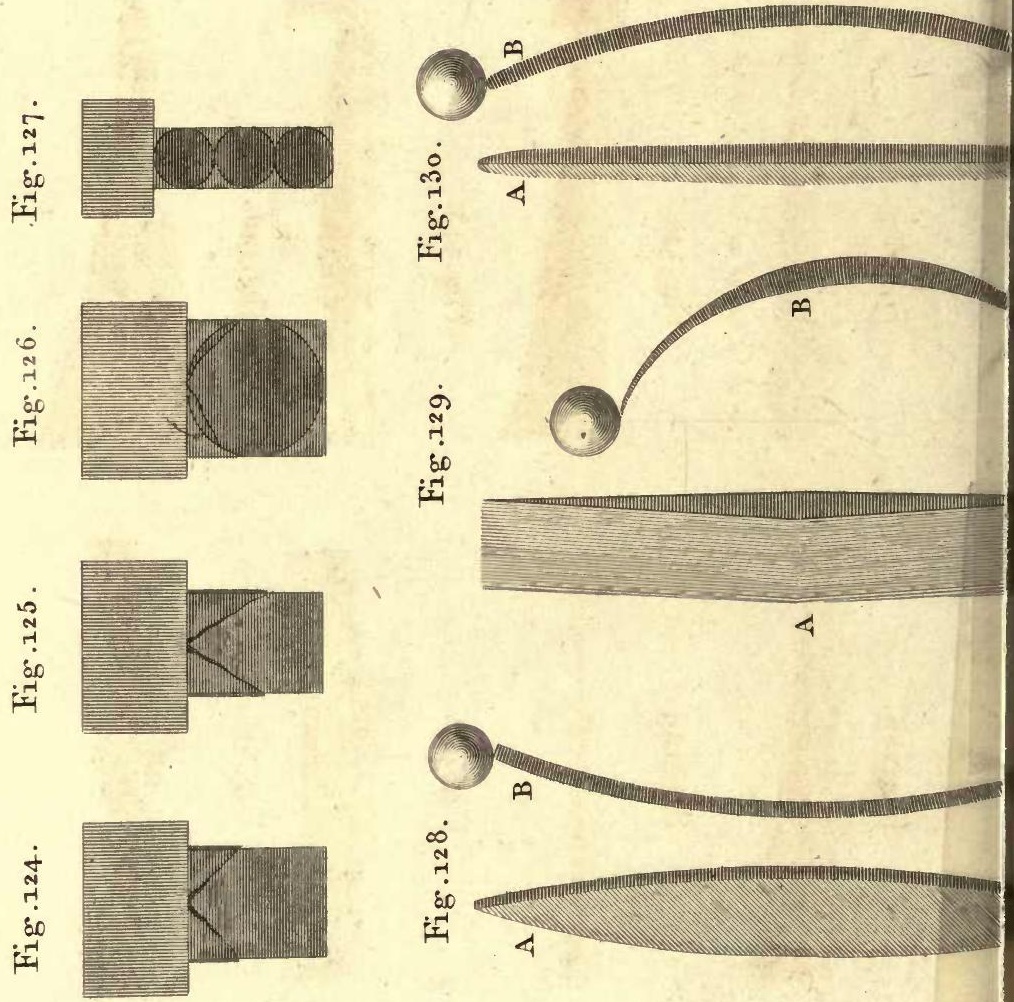

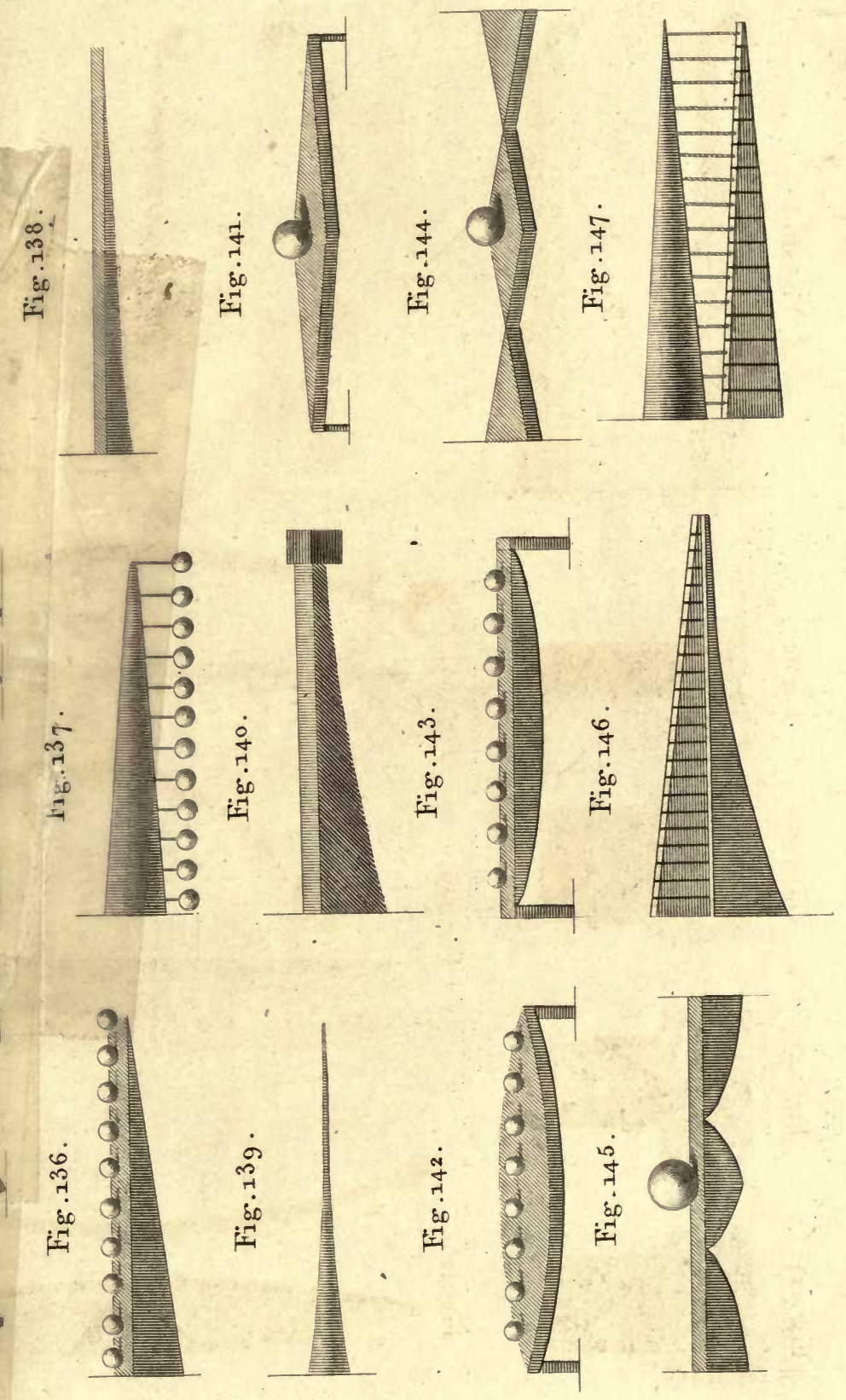



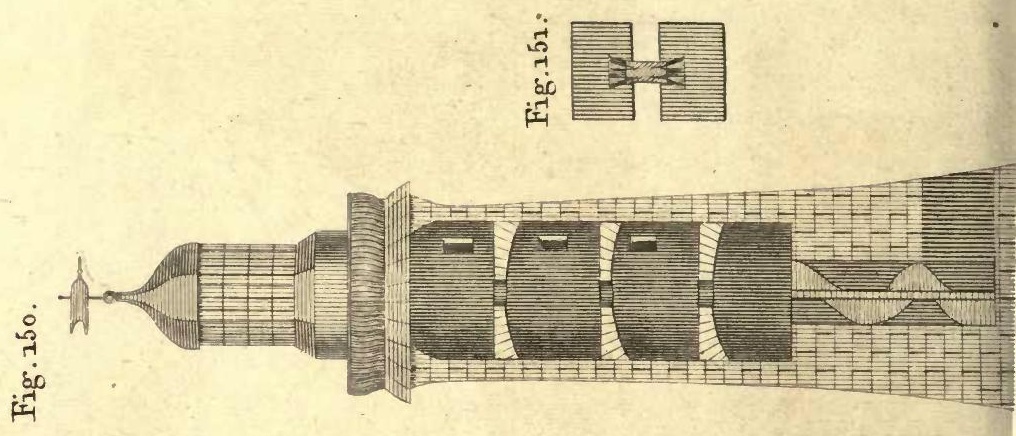

궁
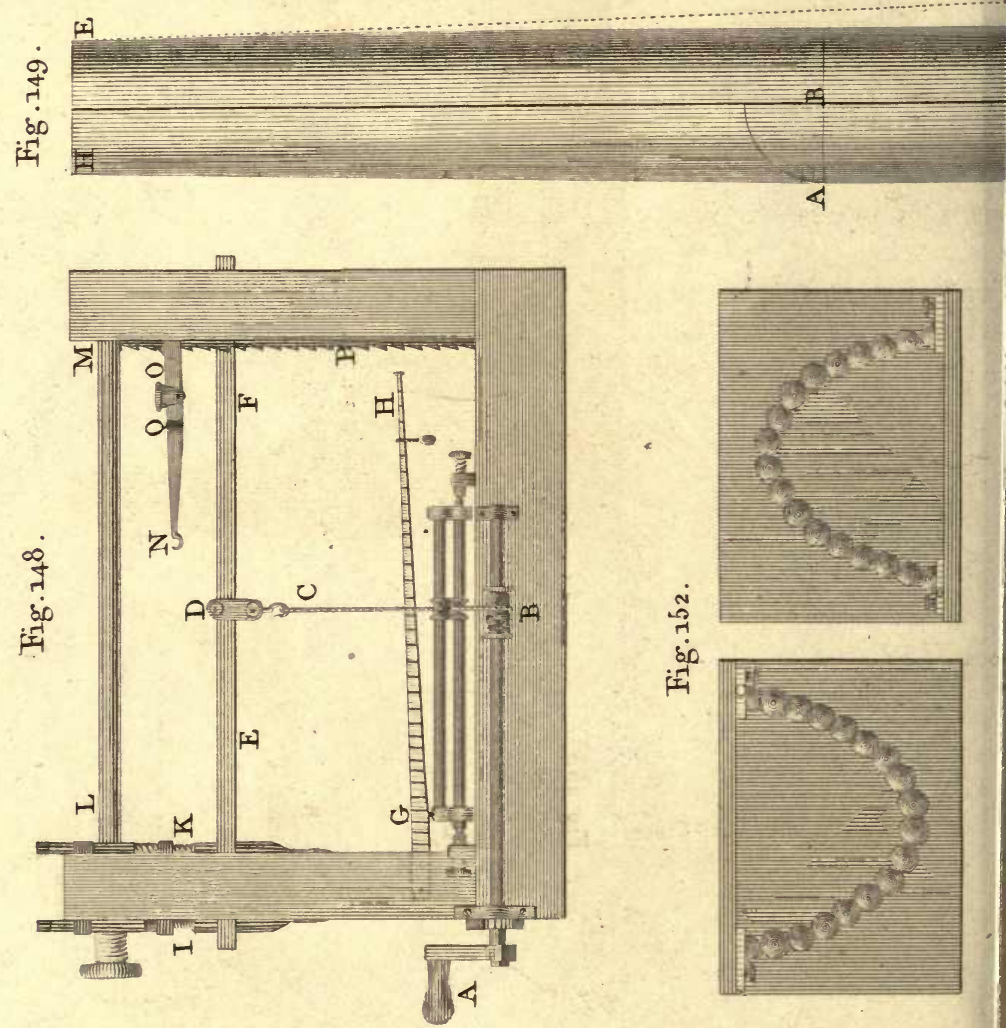

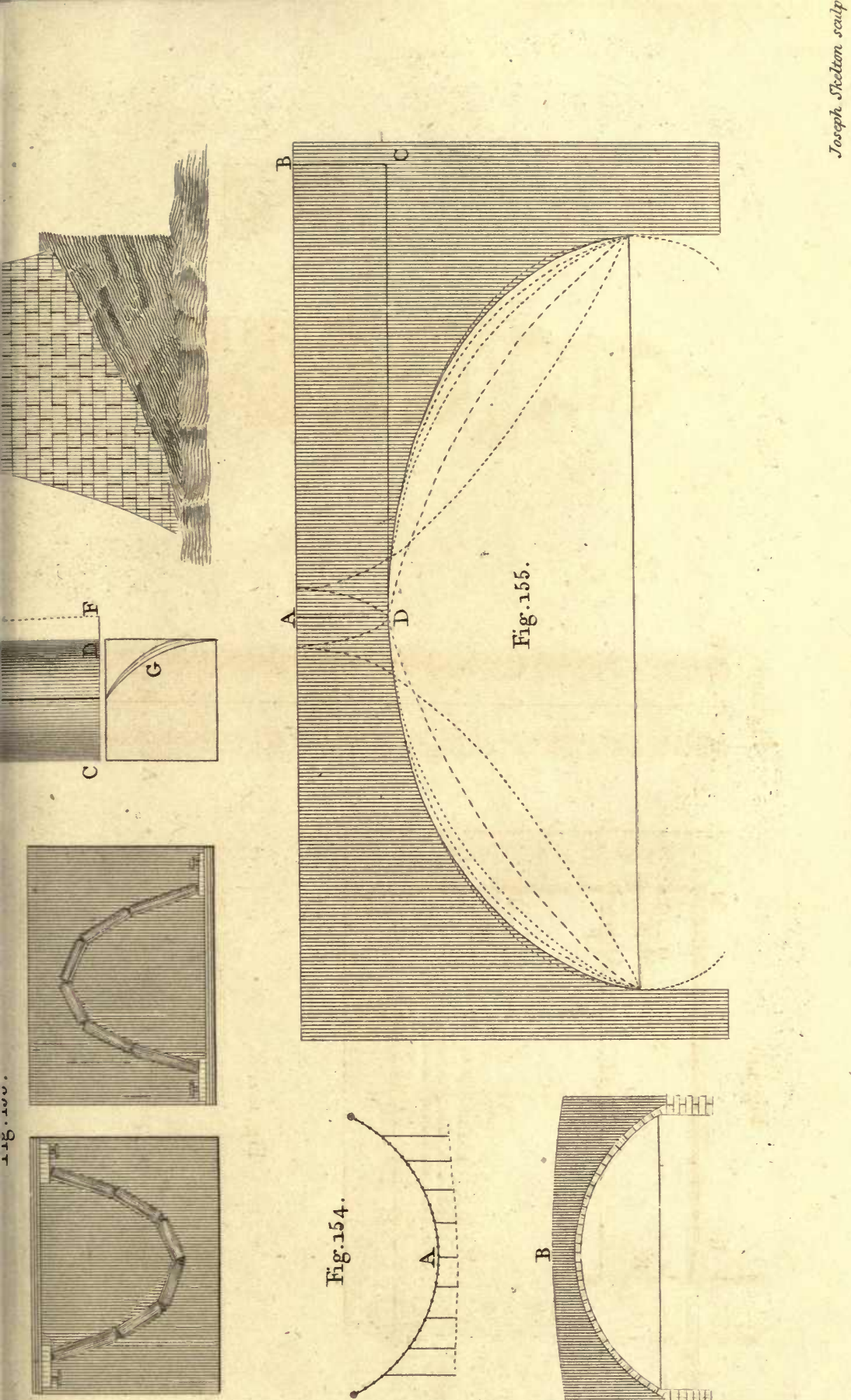




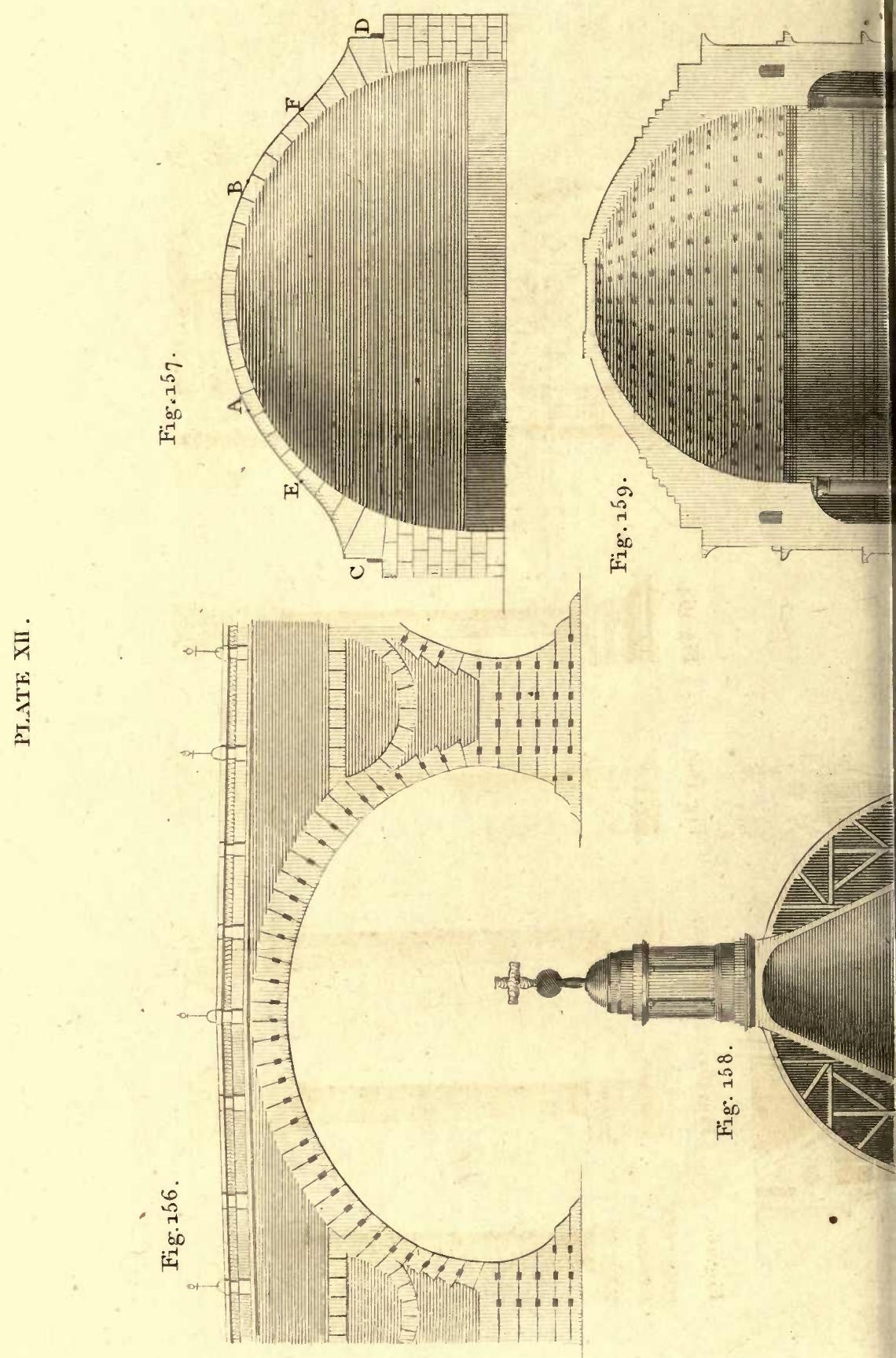




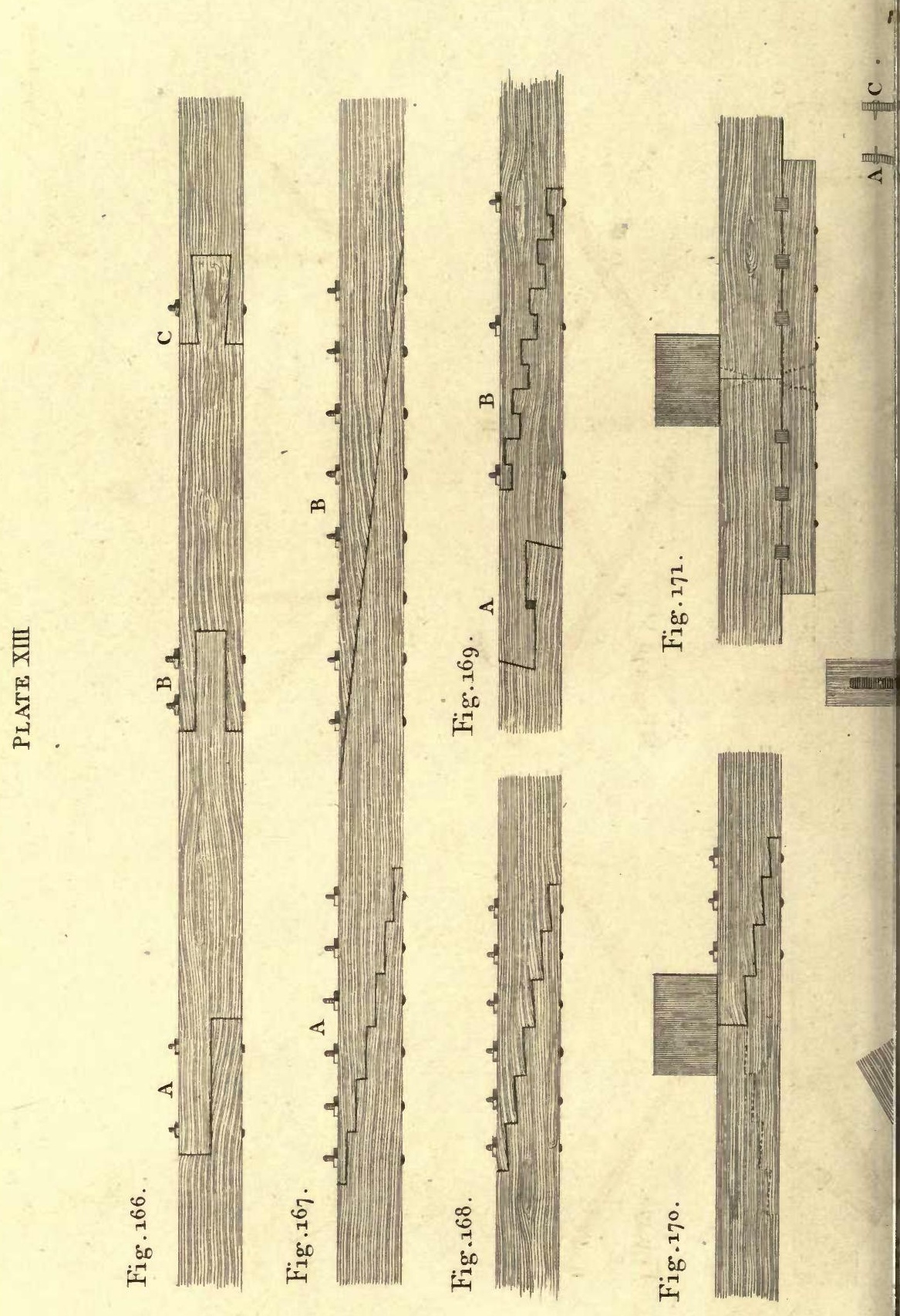



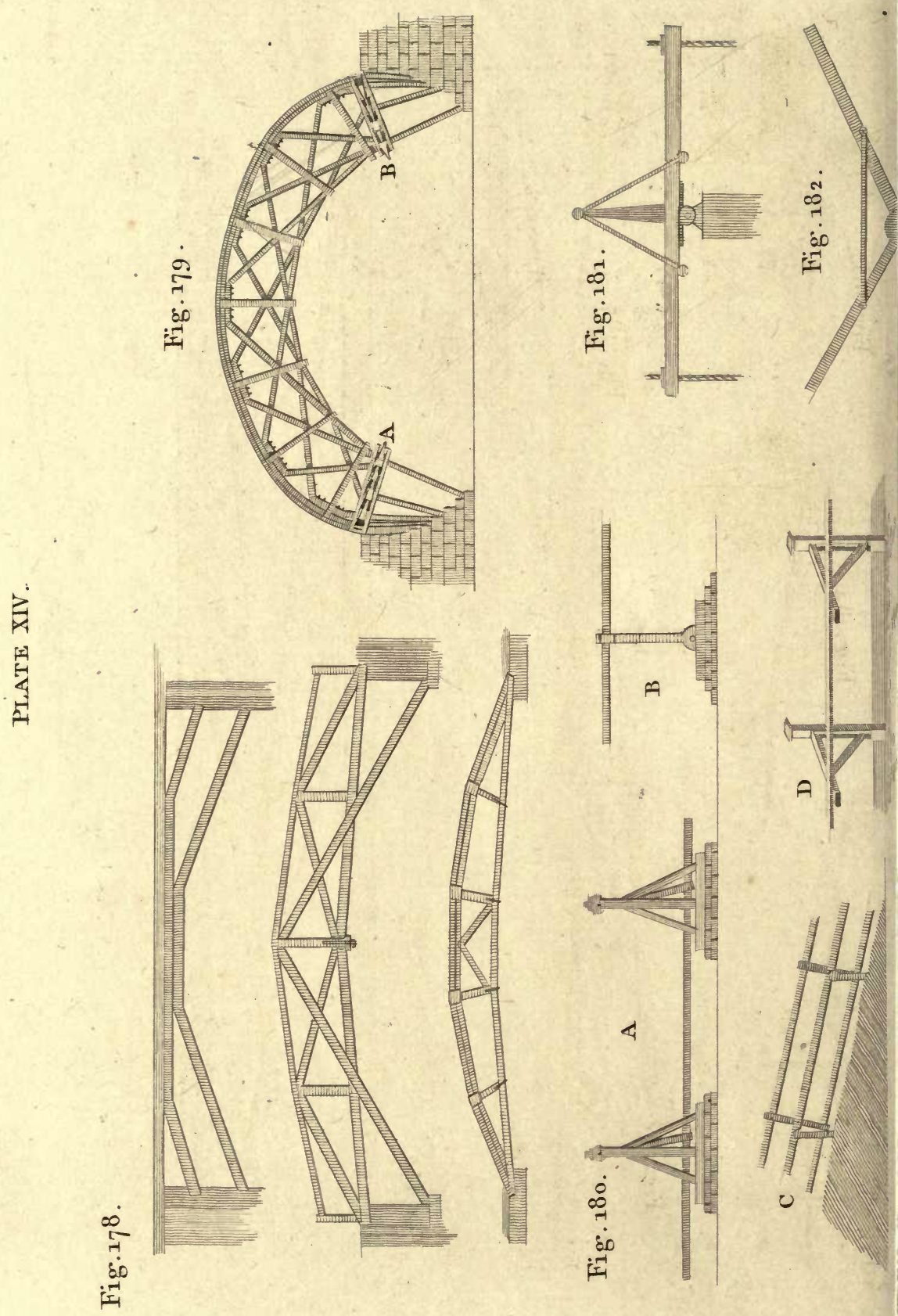




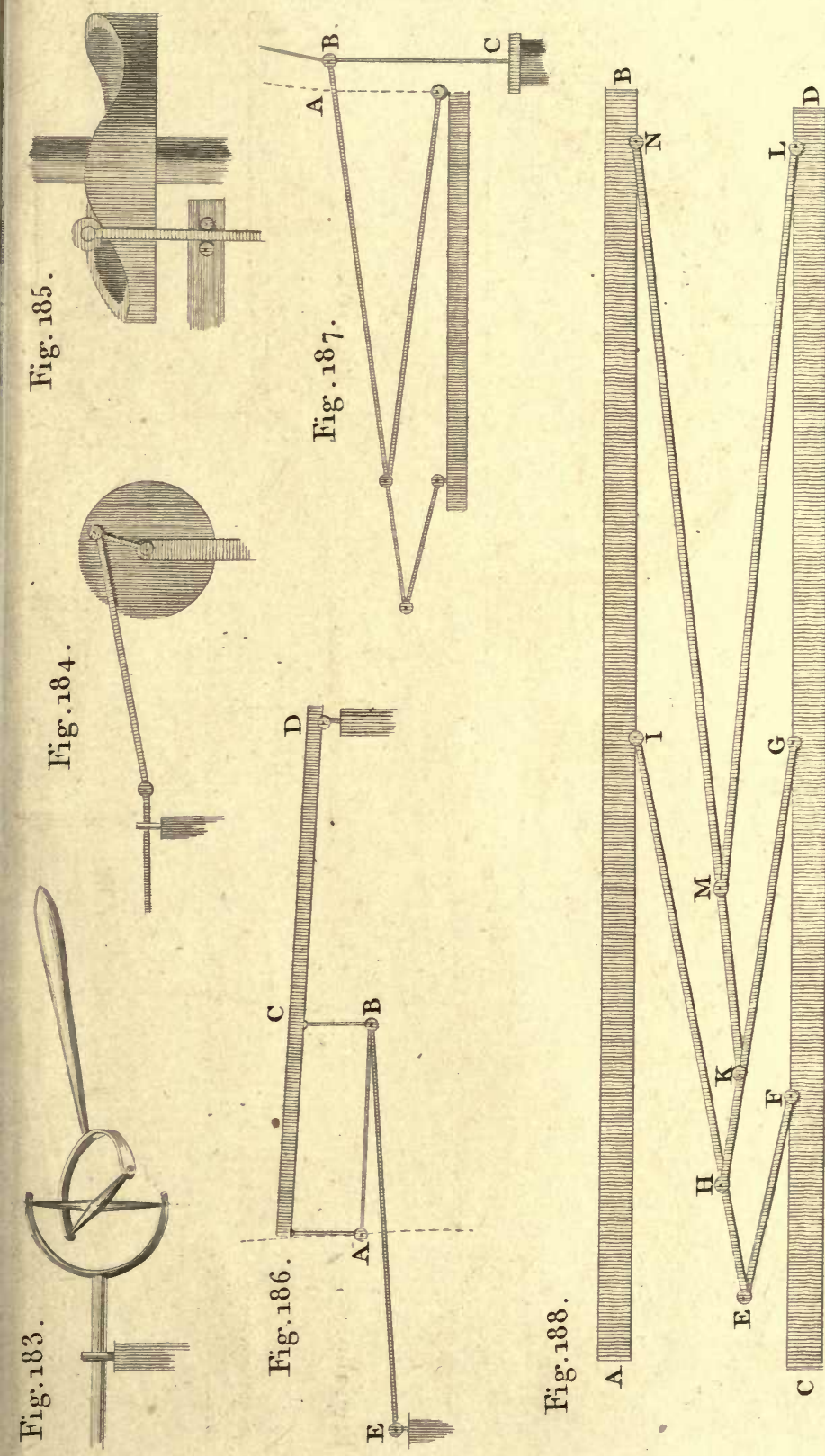

है

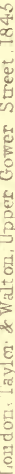





$$
15
$$

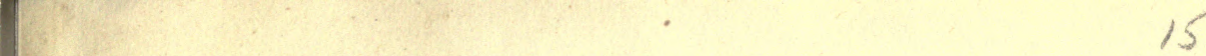



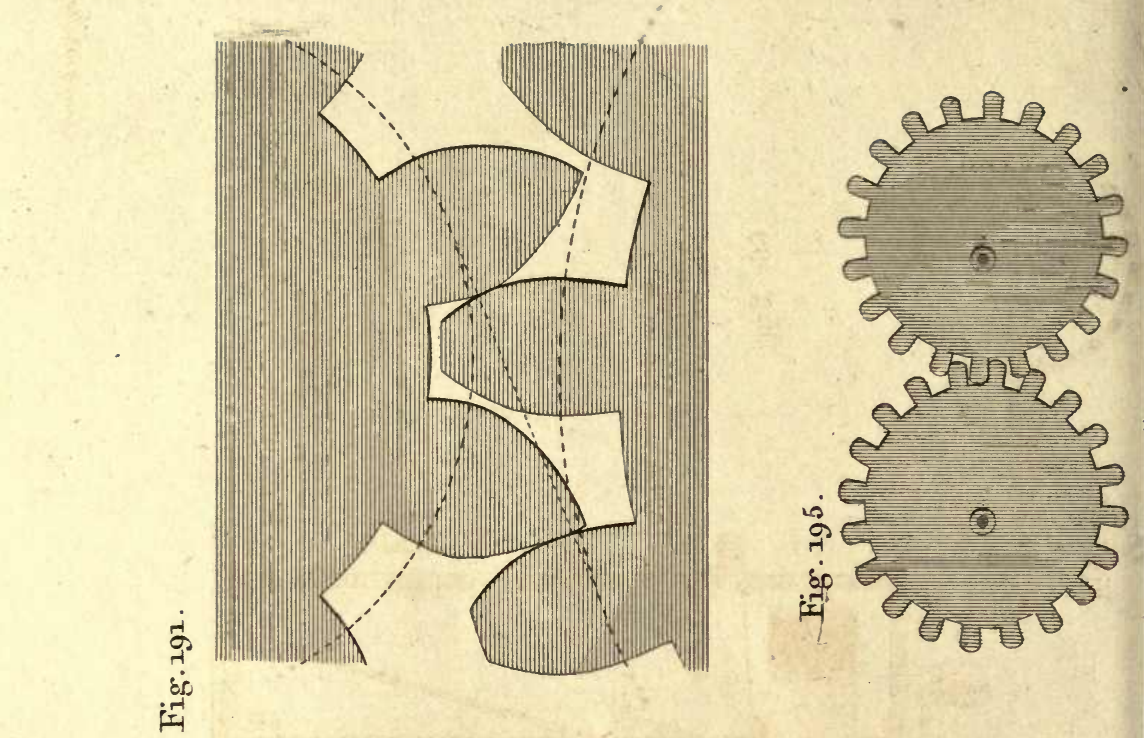

若
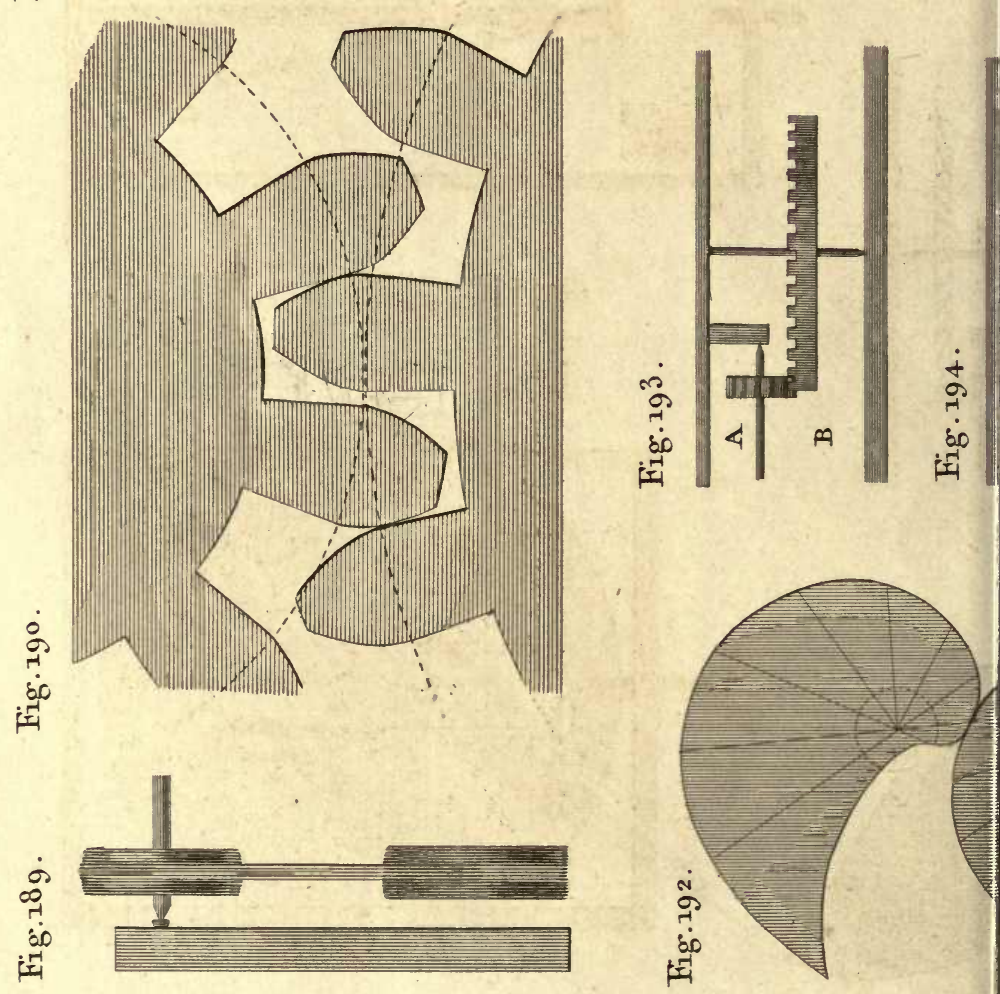


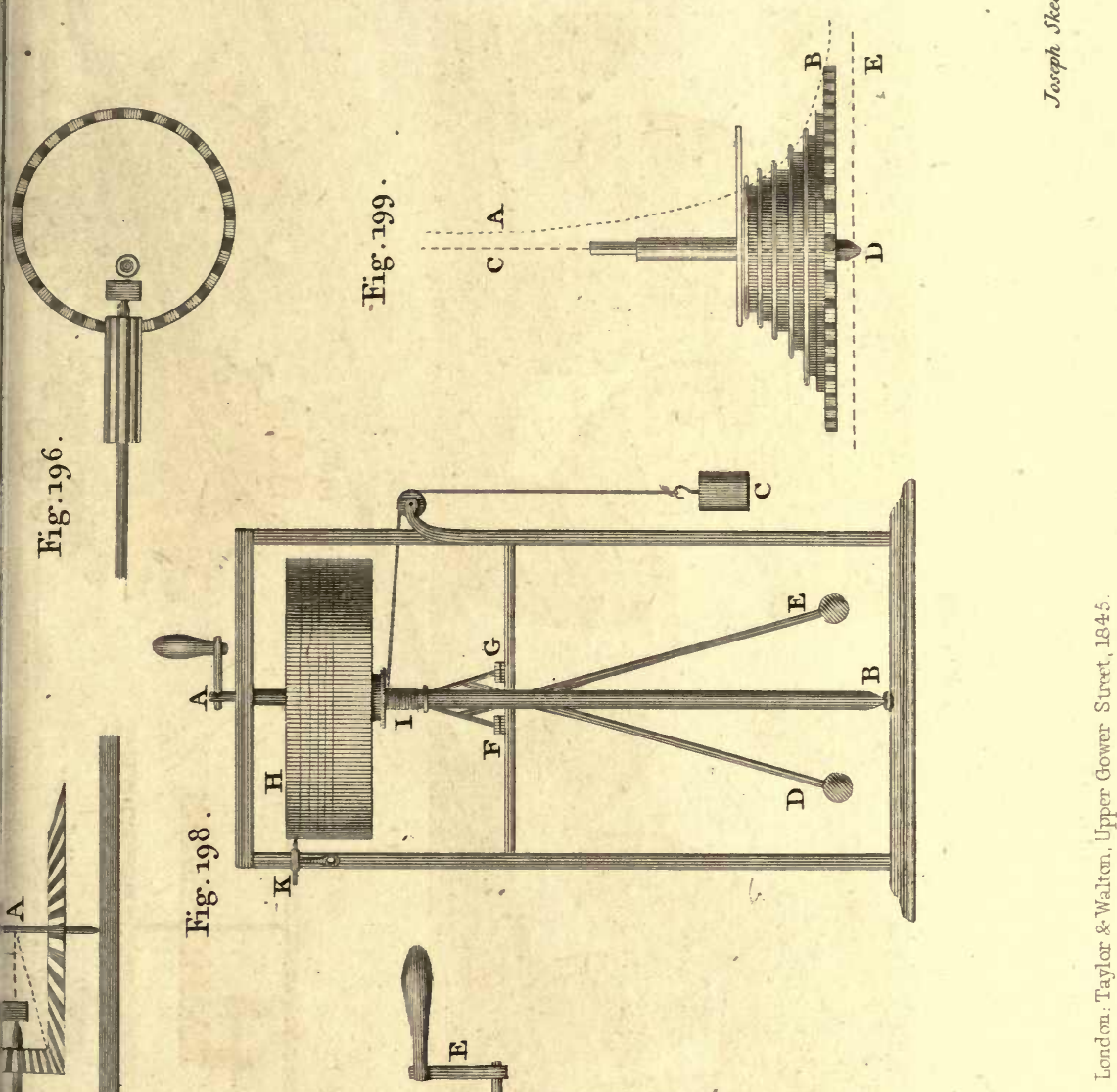





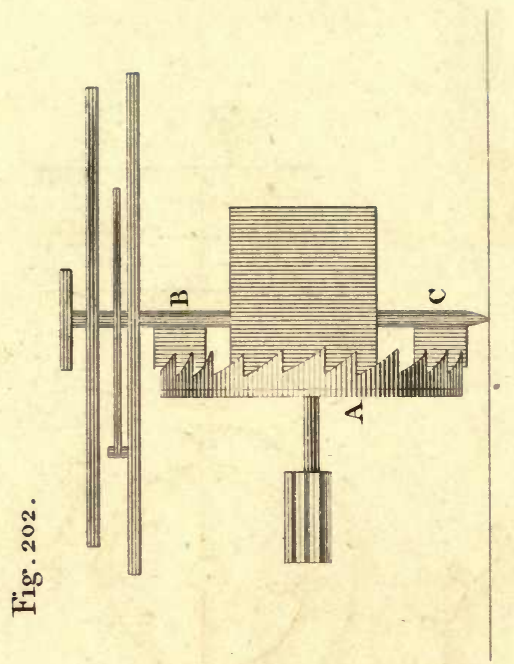

近
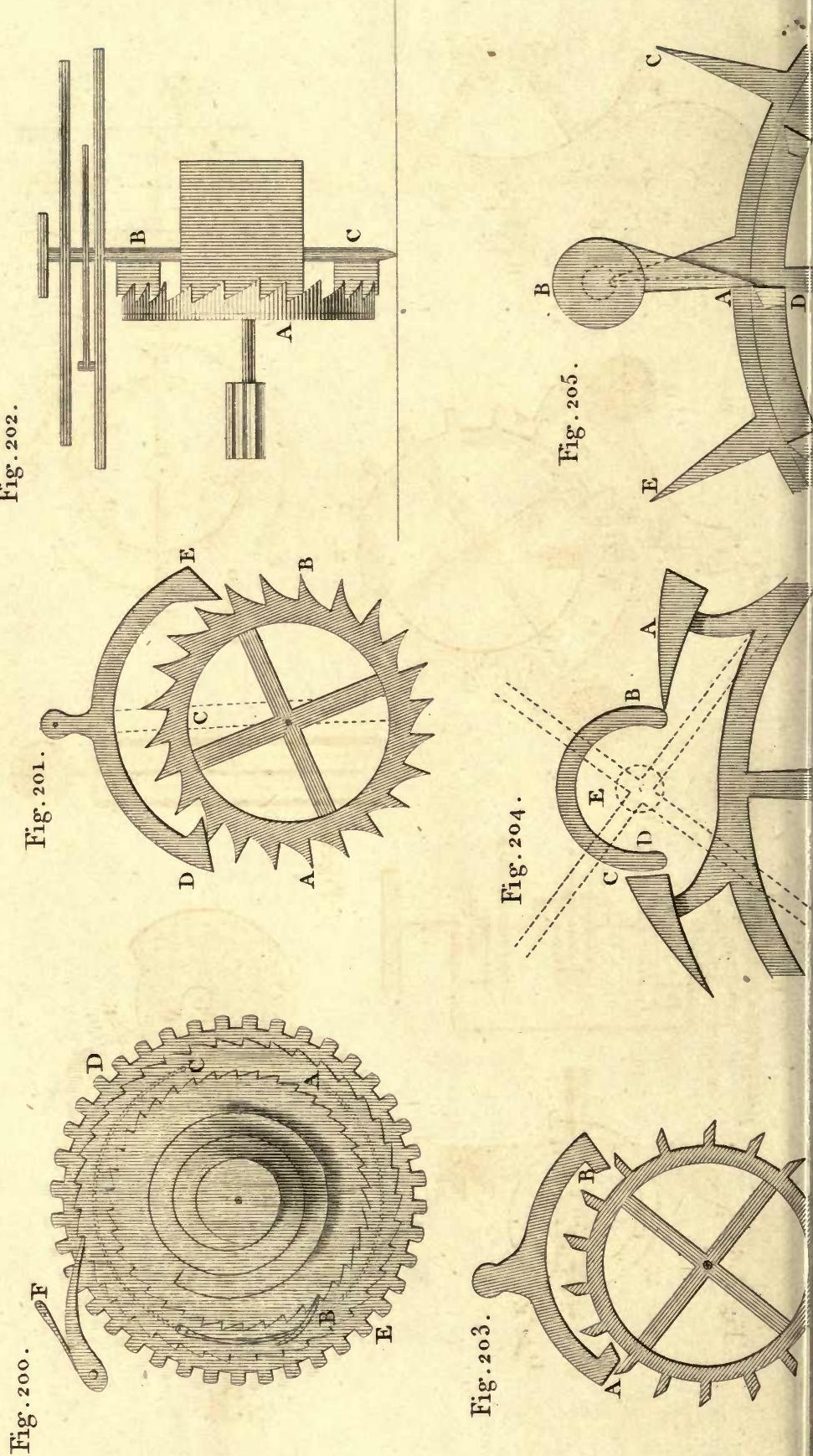


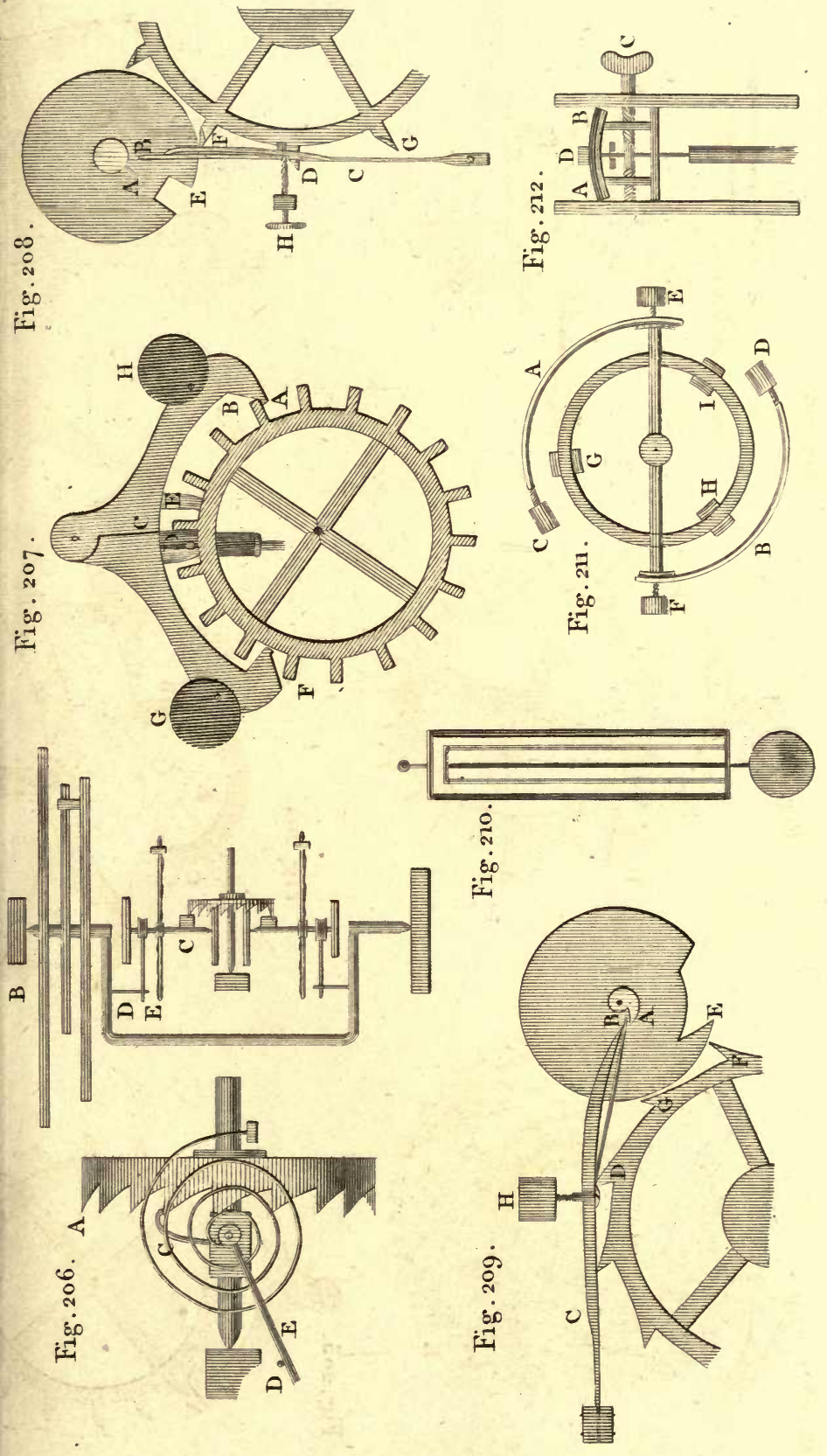





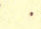



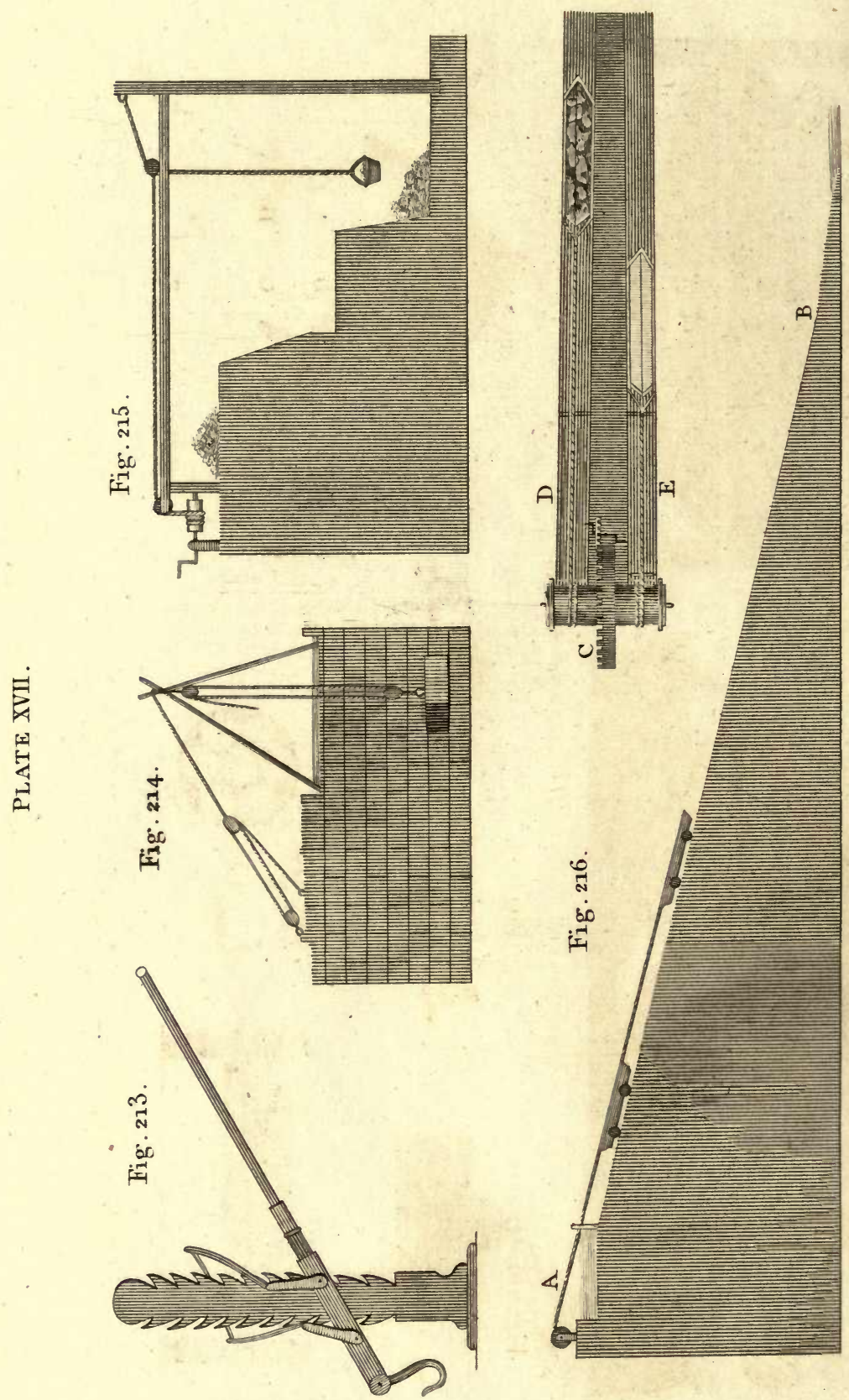

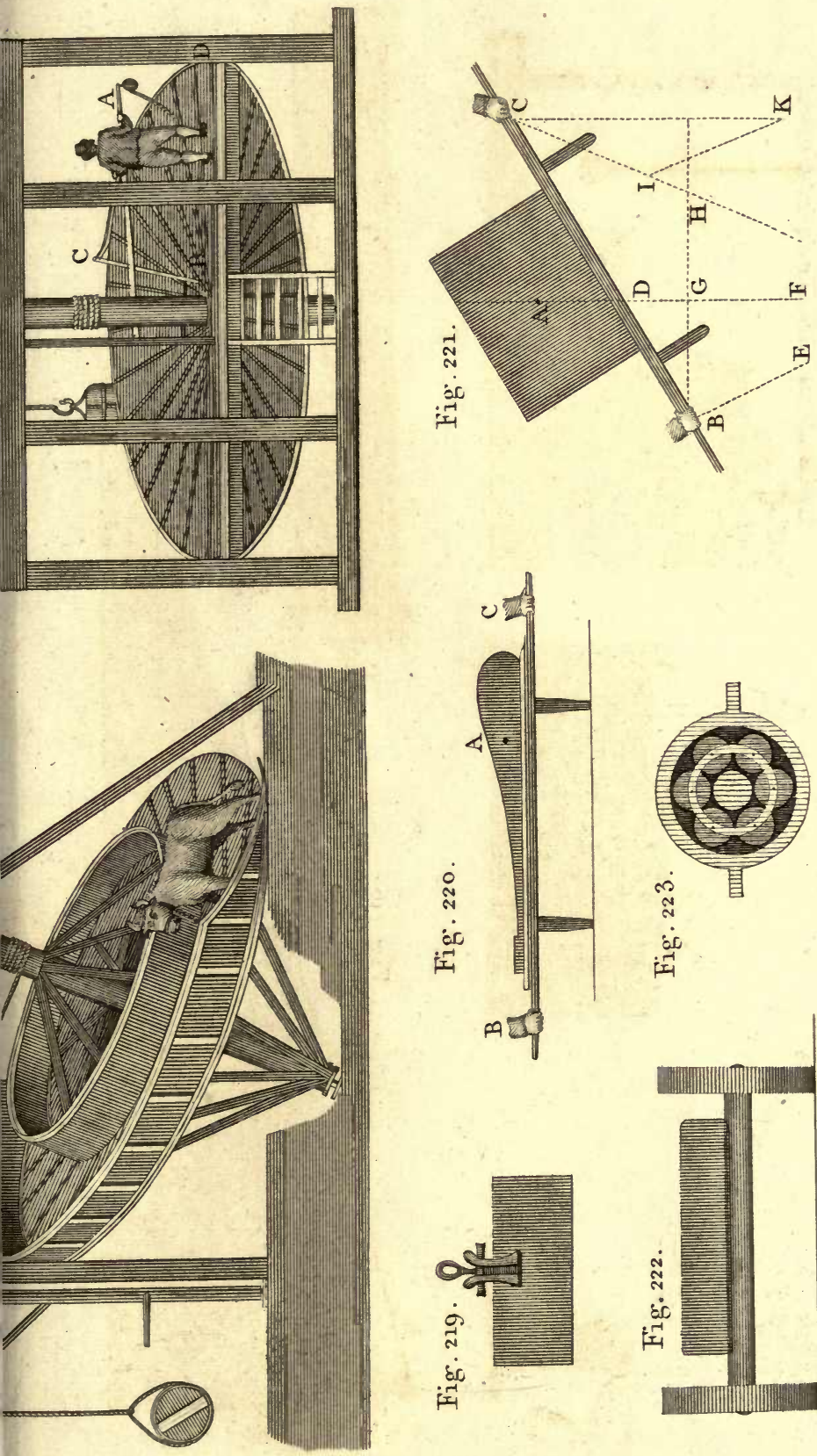

$\approx$

N

is

iन्य
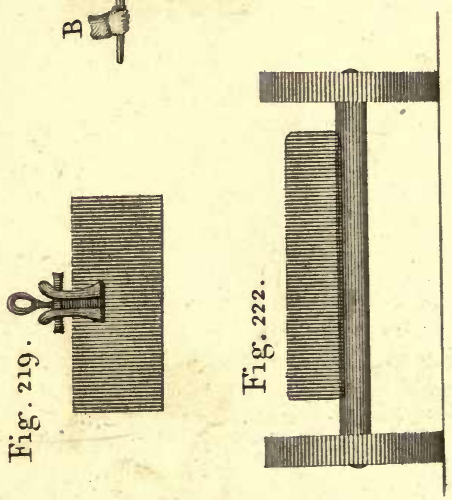



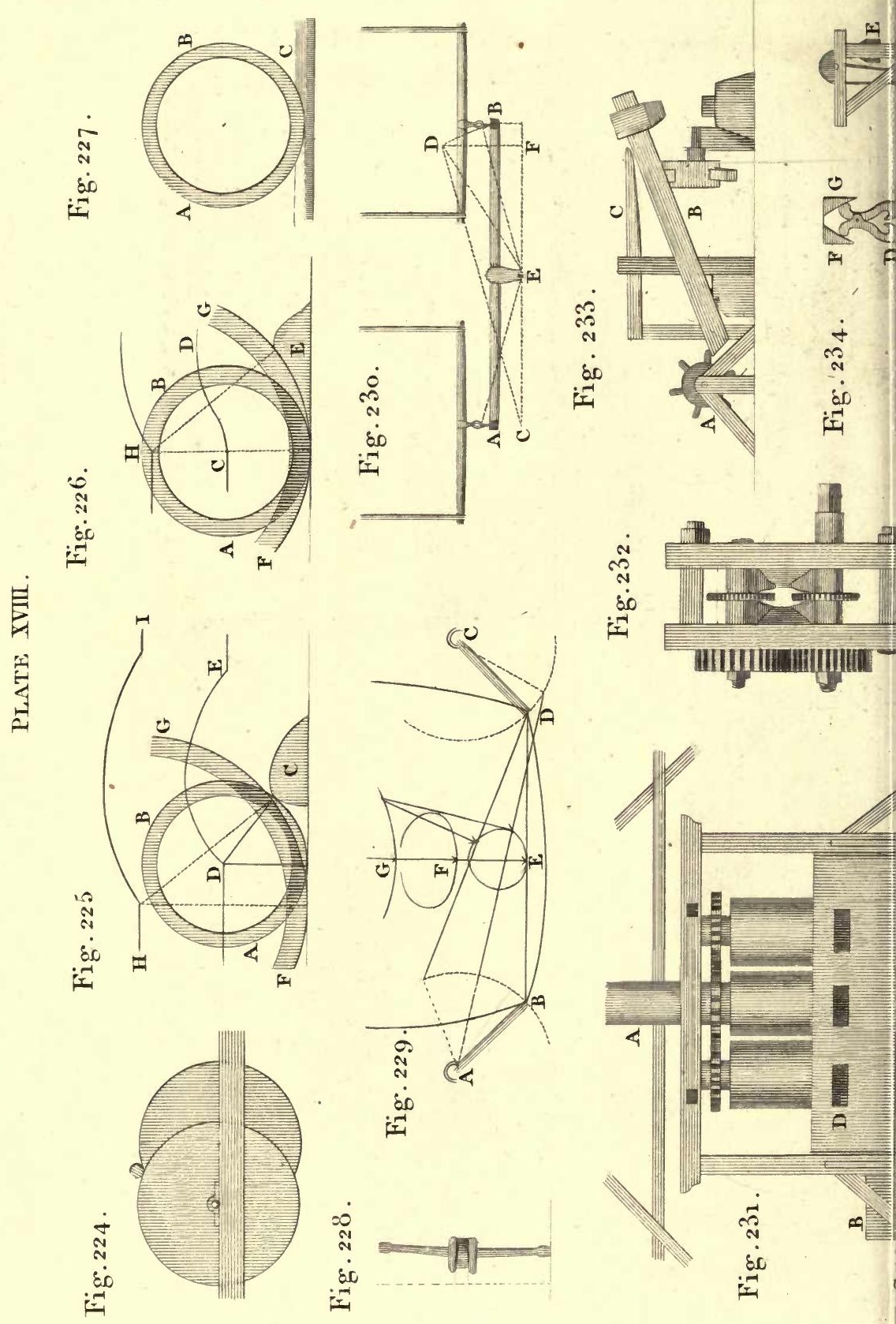




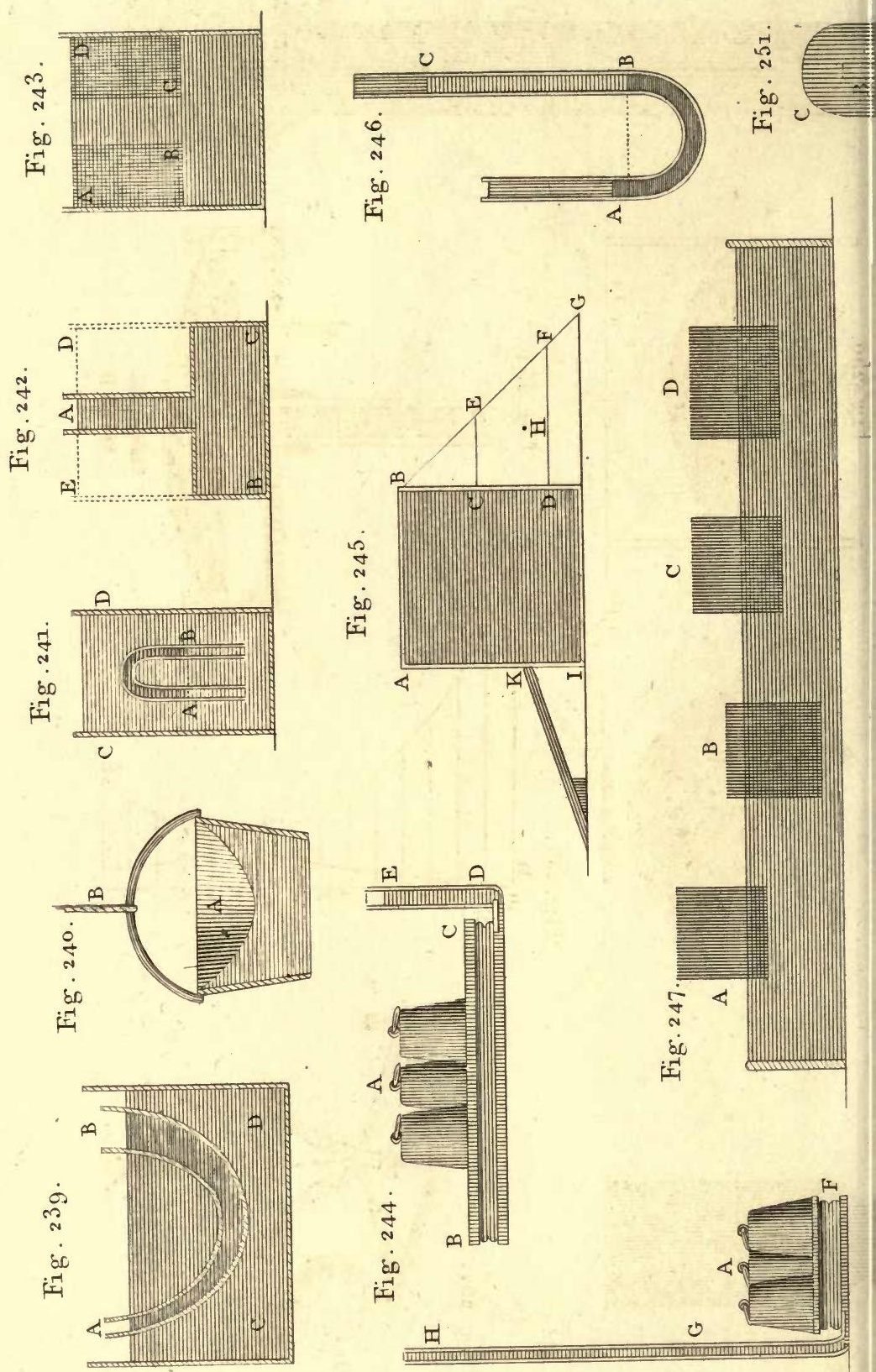



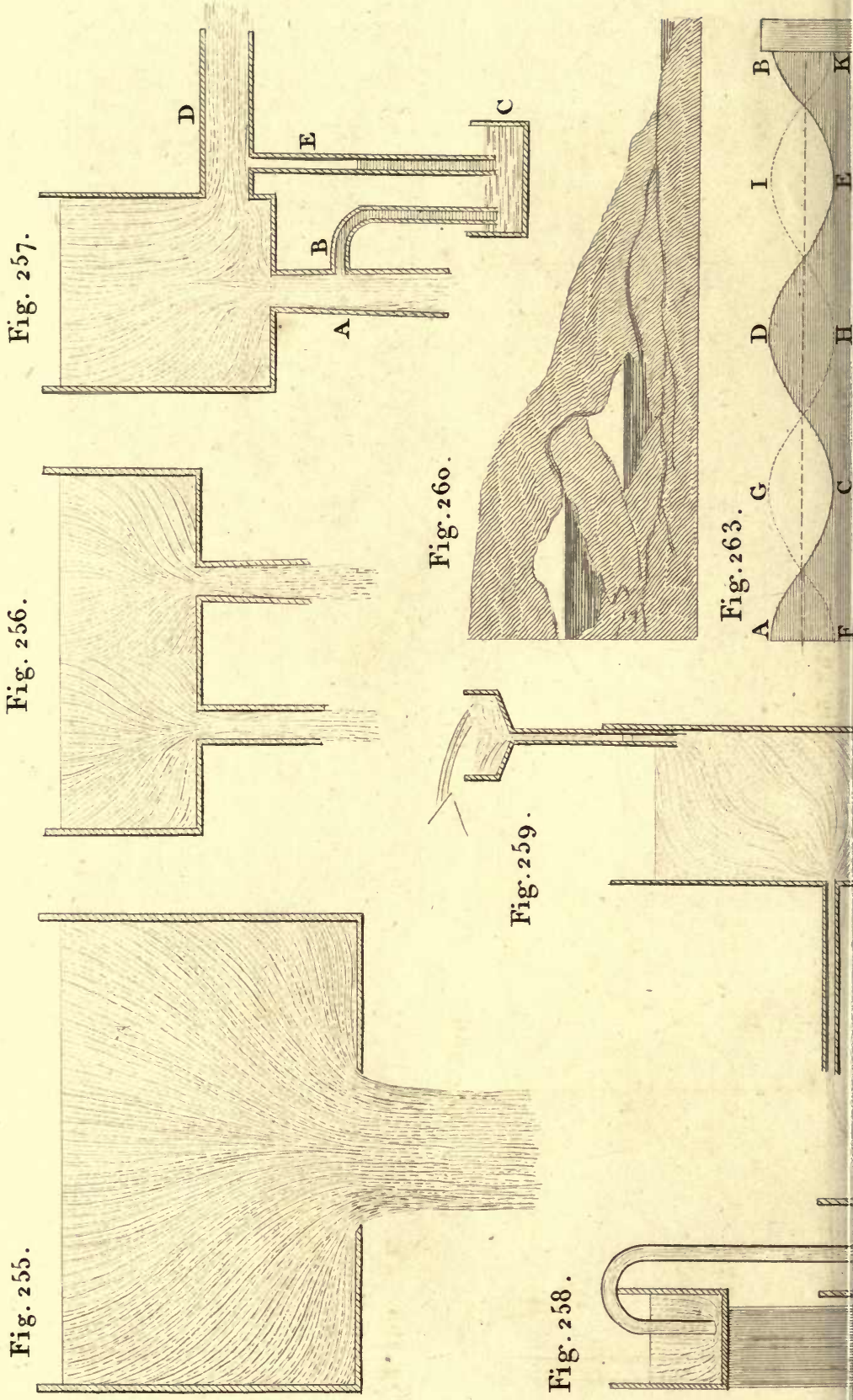



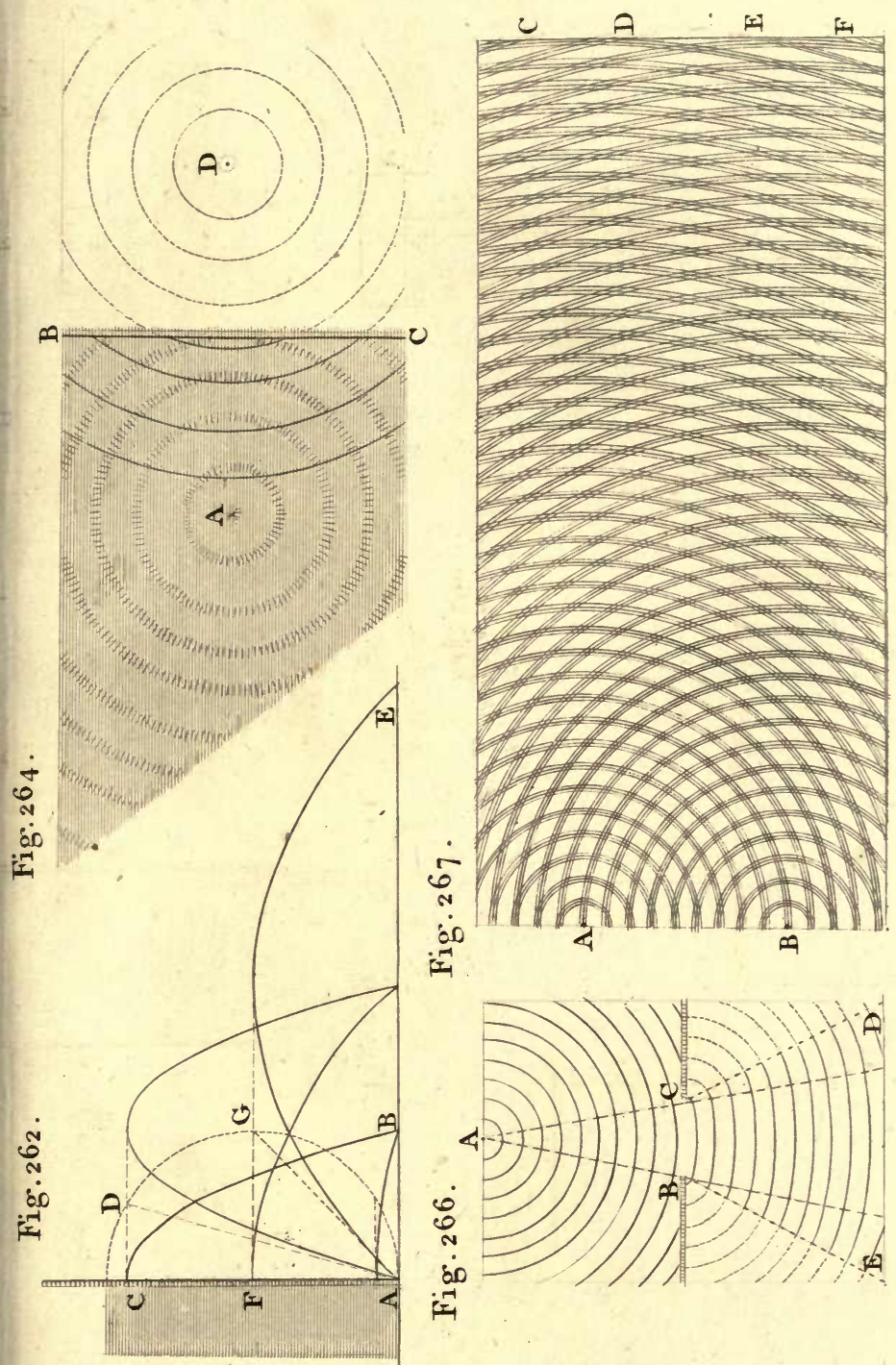

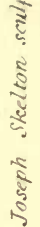
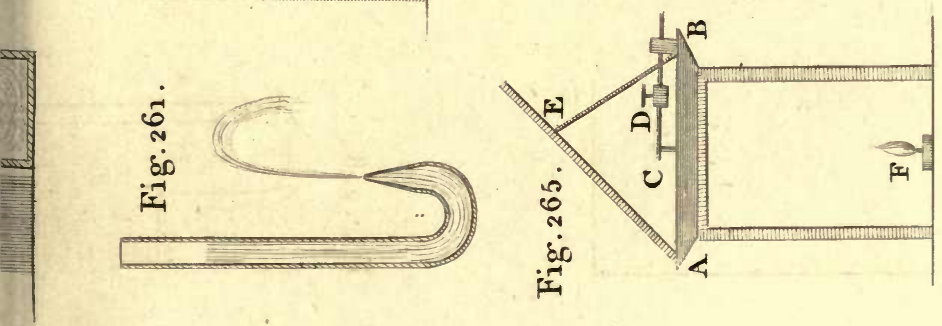



\section{$x$}




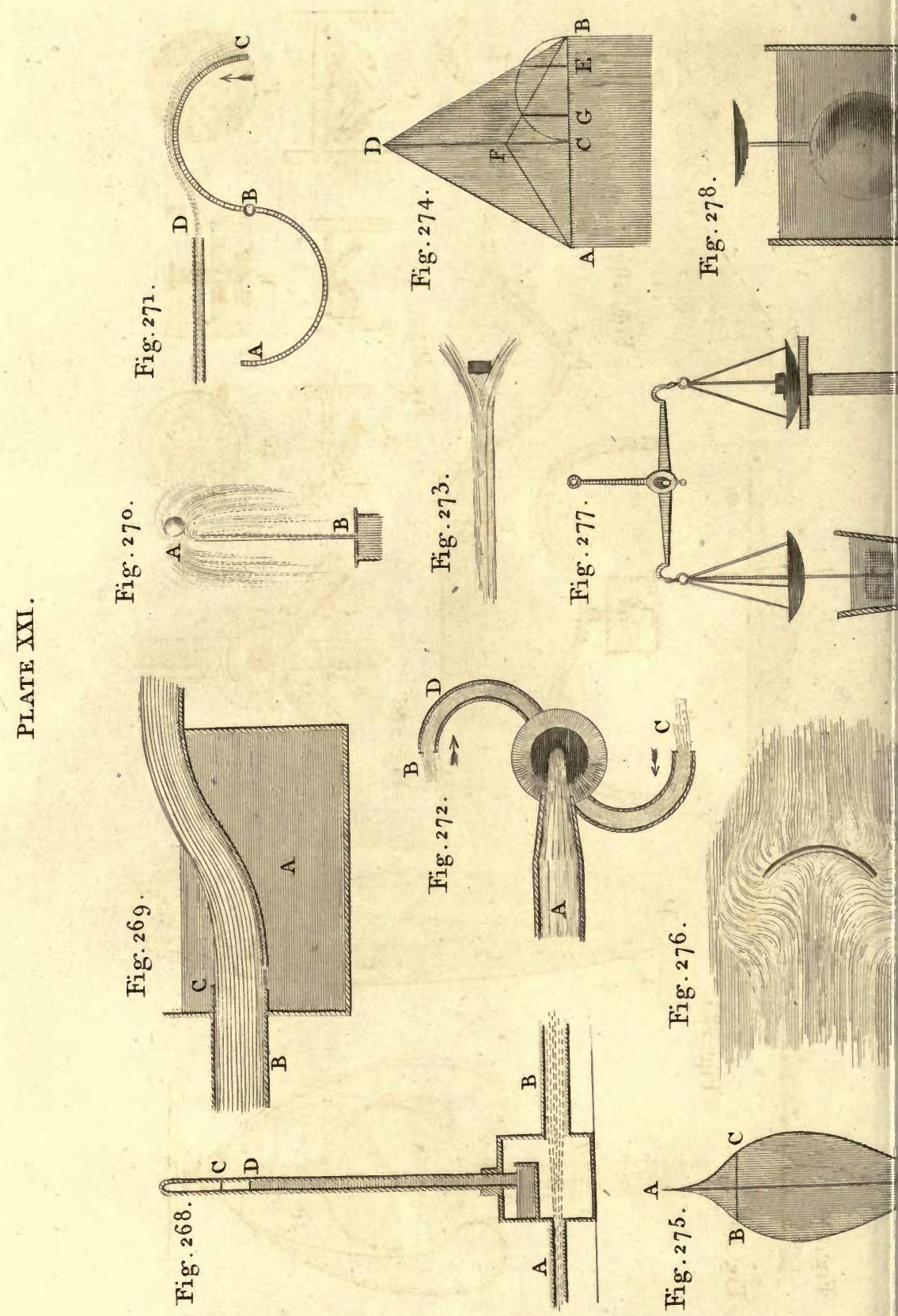




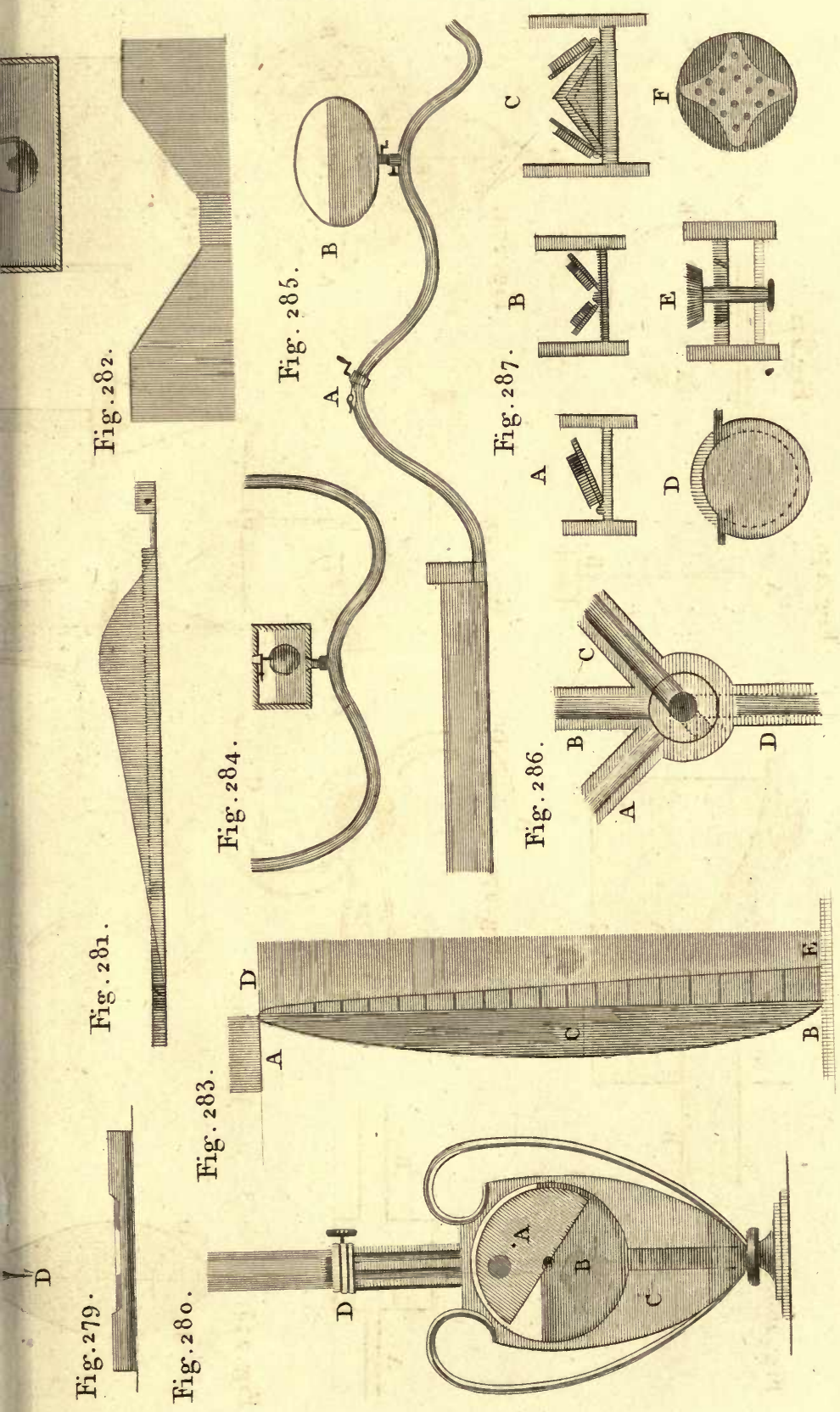

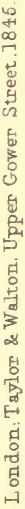




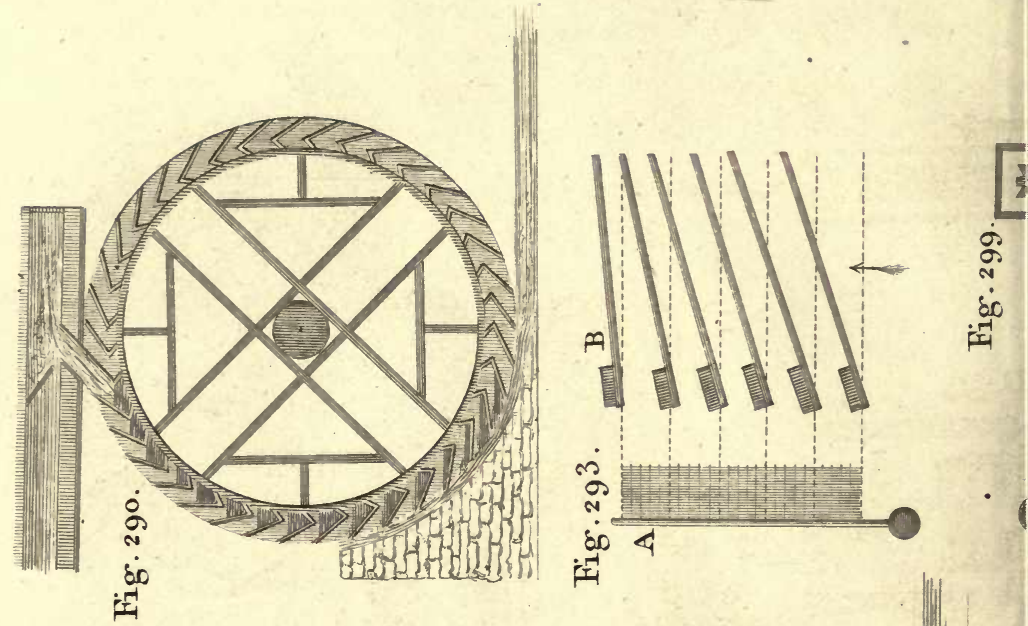

곡
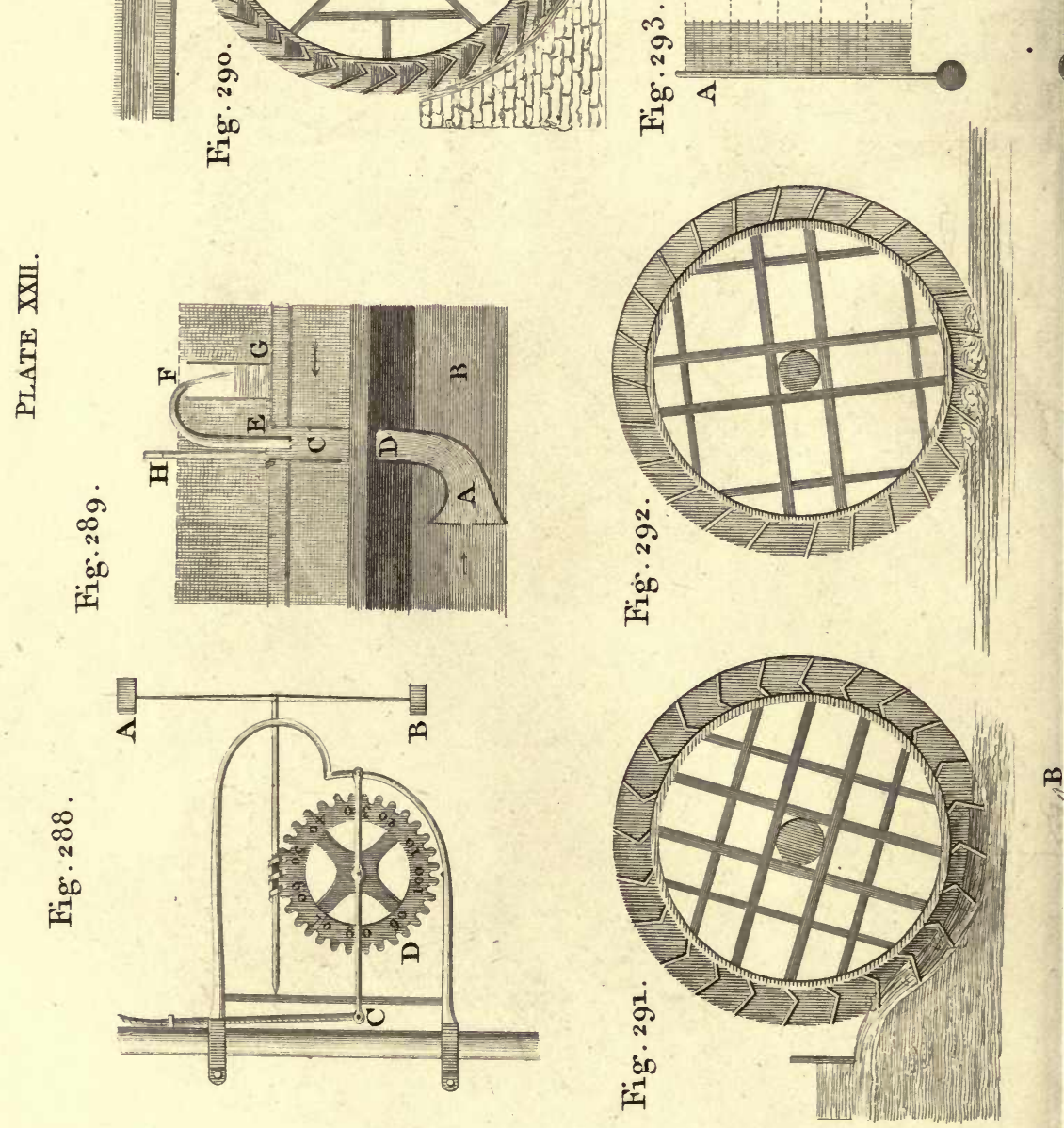



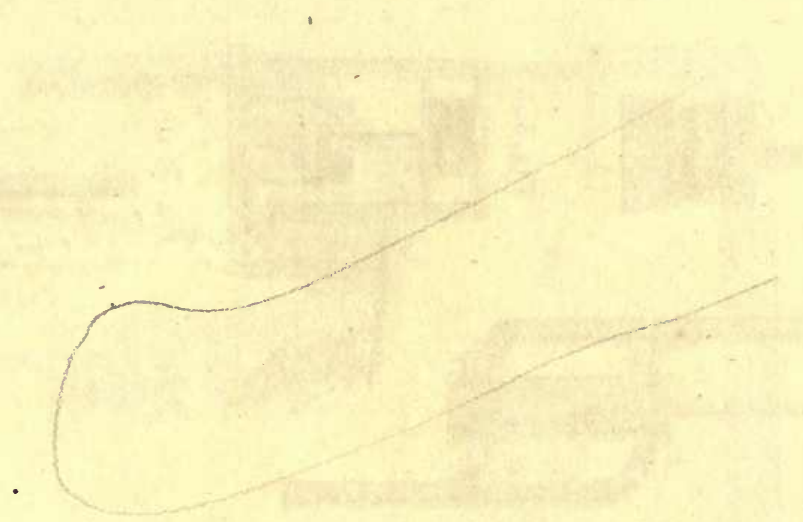



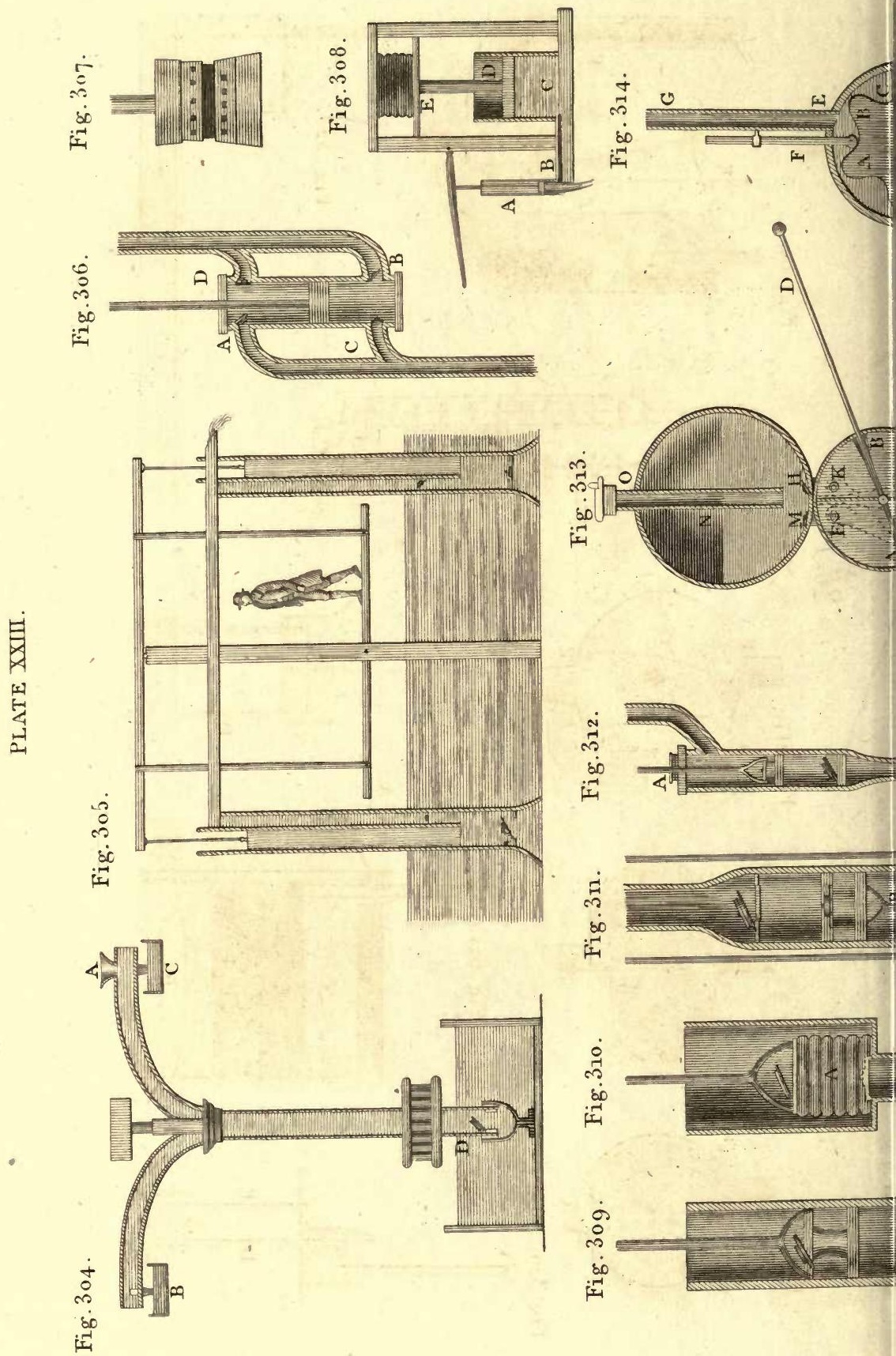


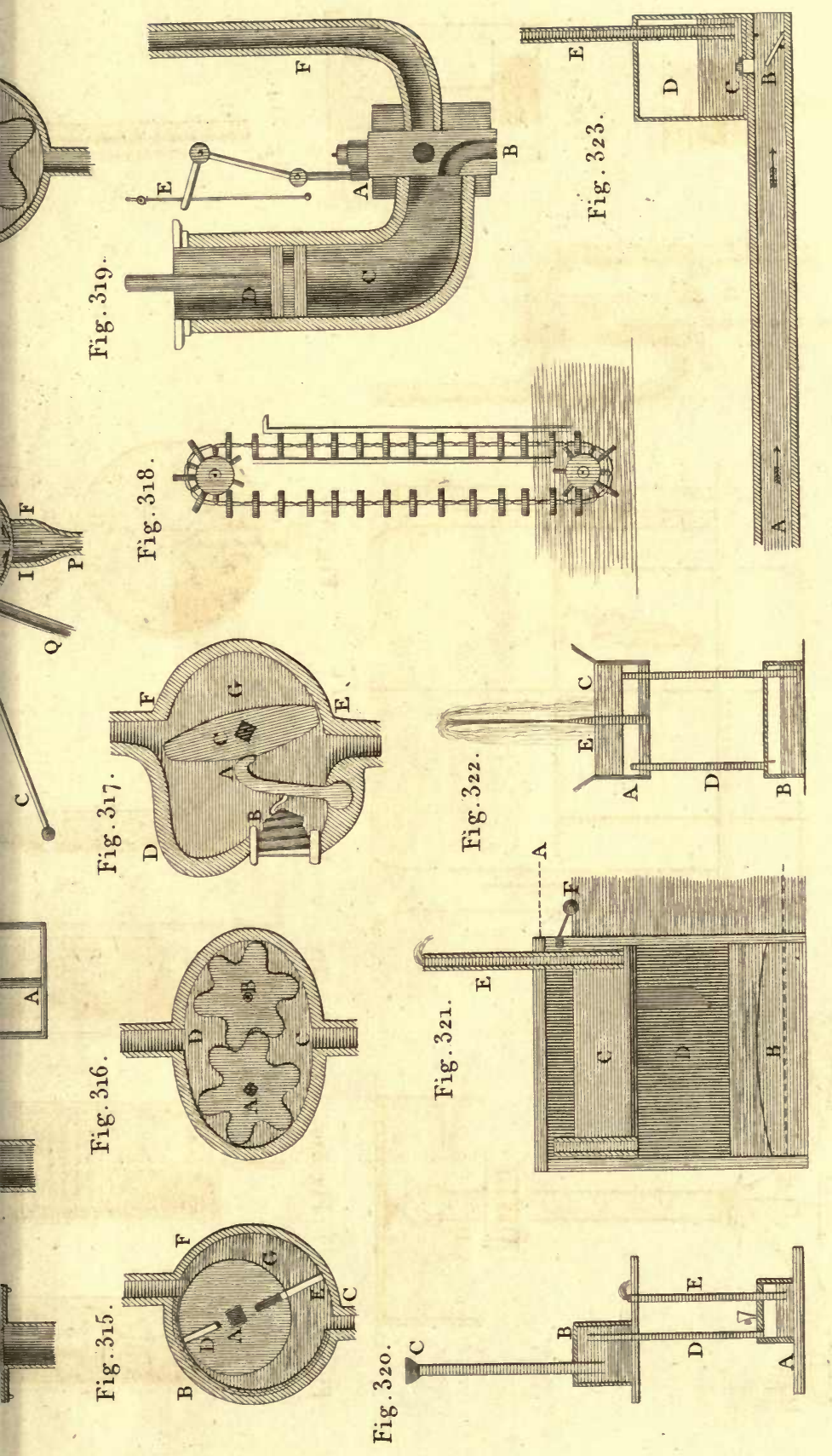




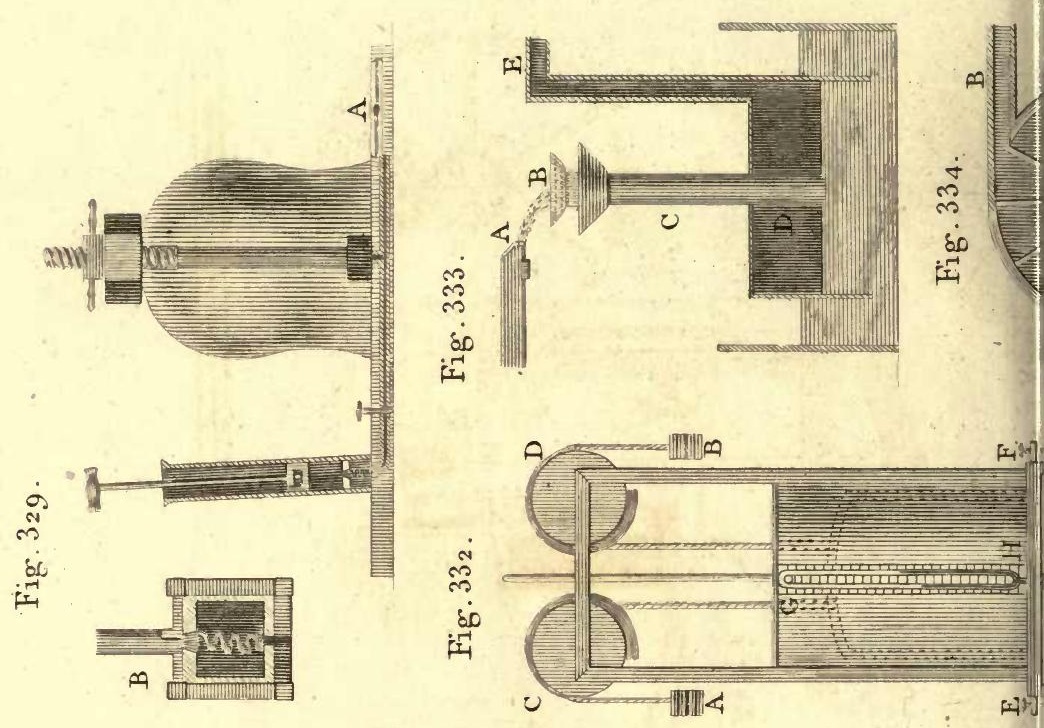

릉

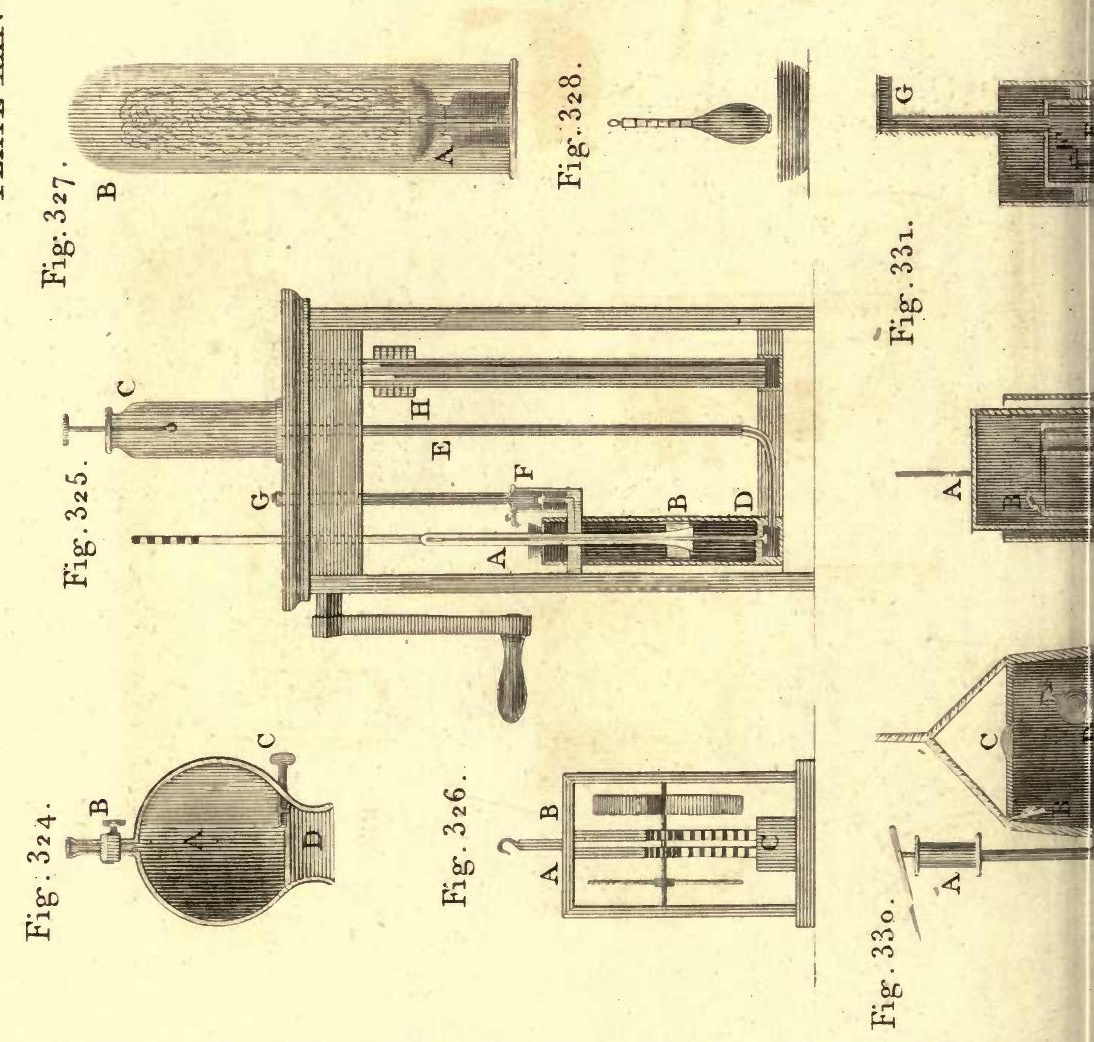




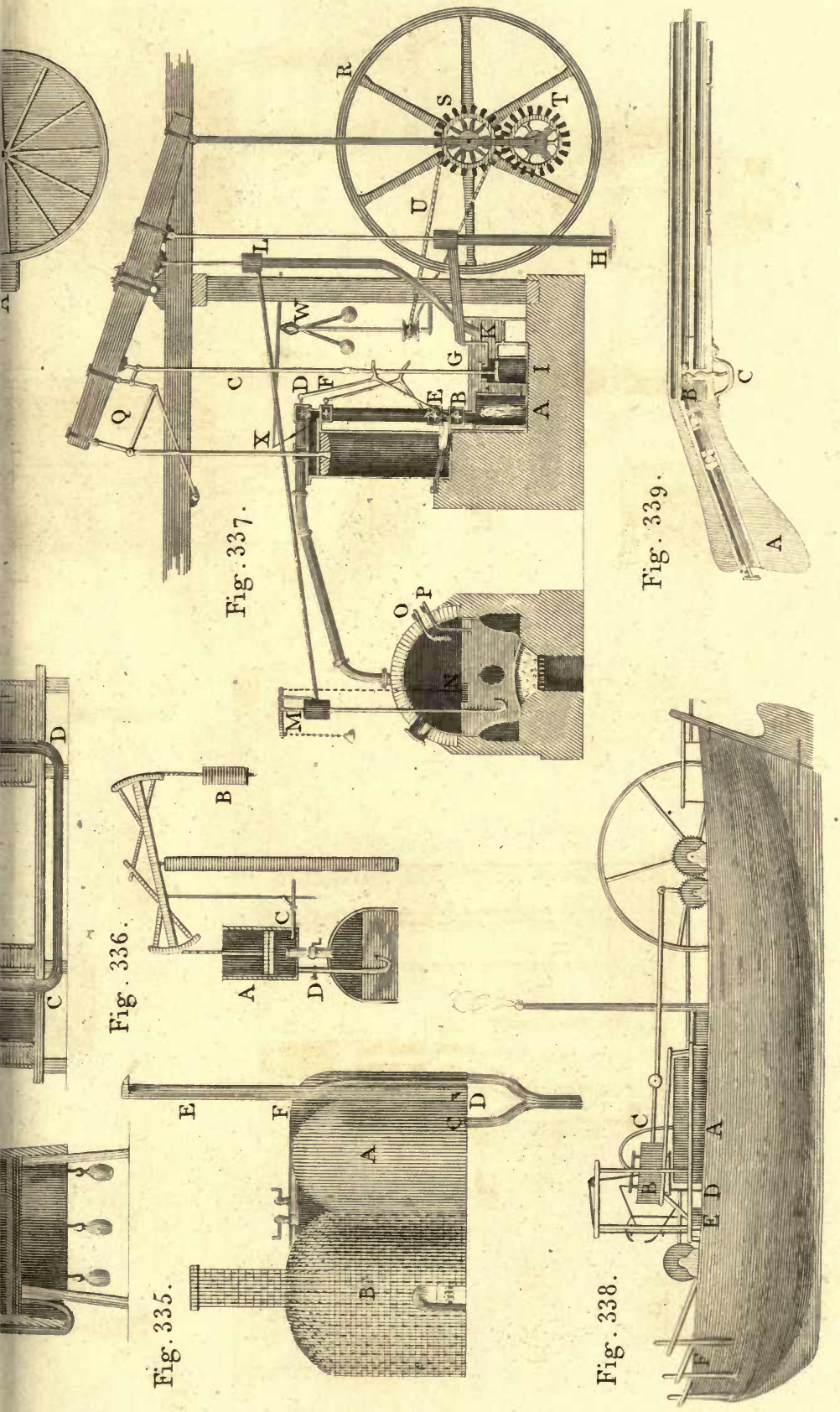



25

is 


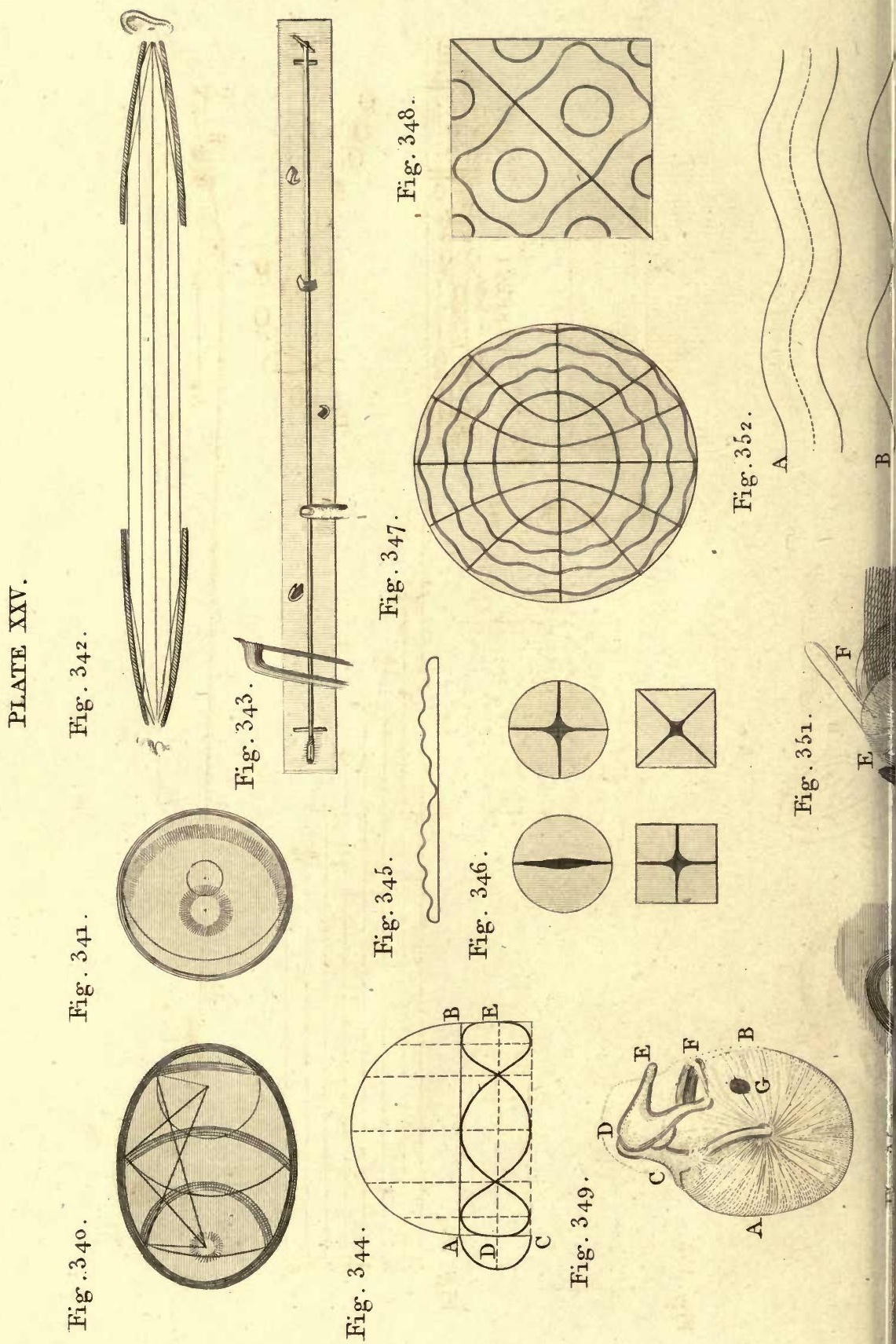



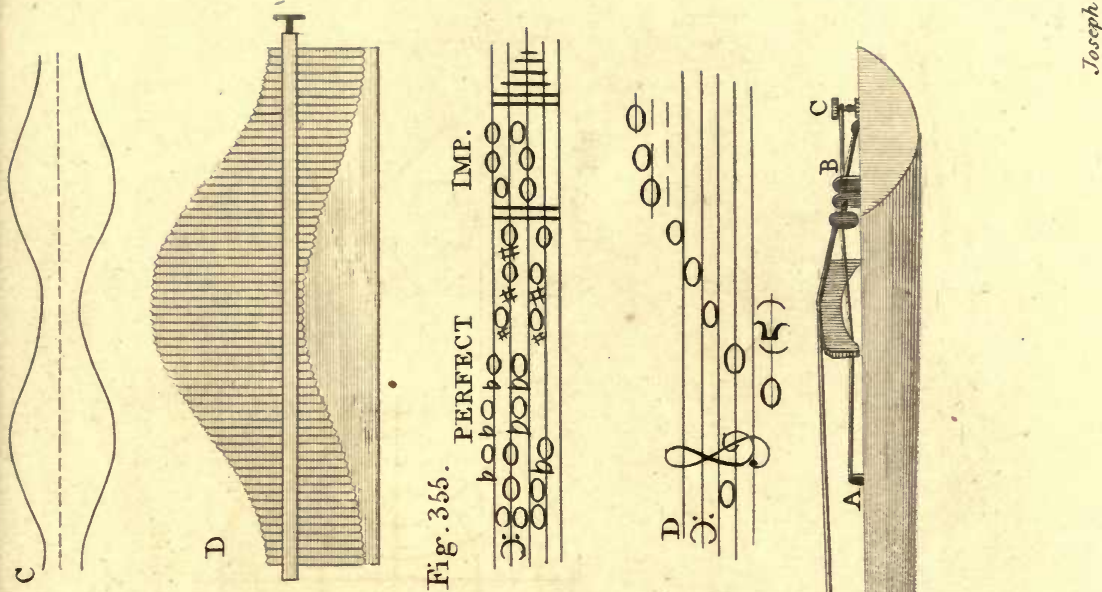

है

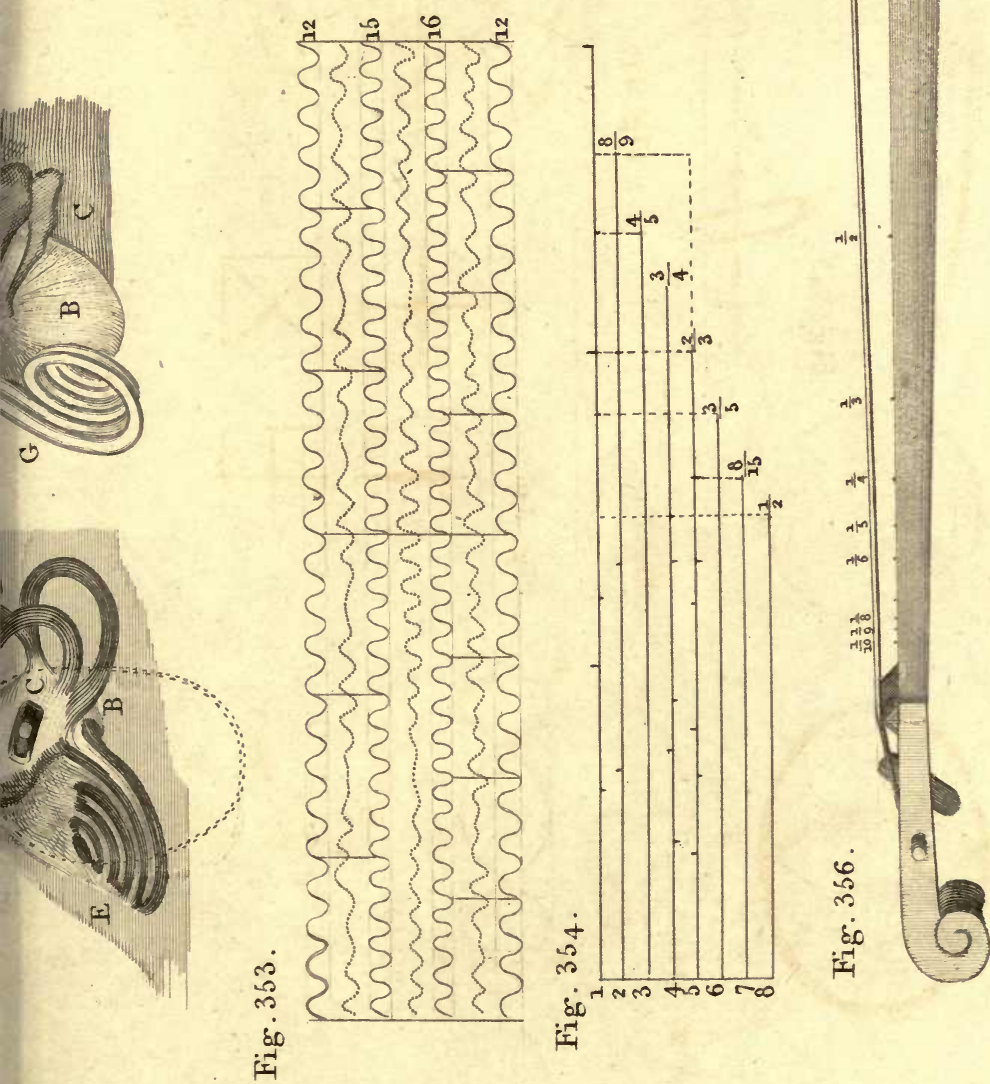

告 

$x^{3} x^{2}=$ 

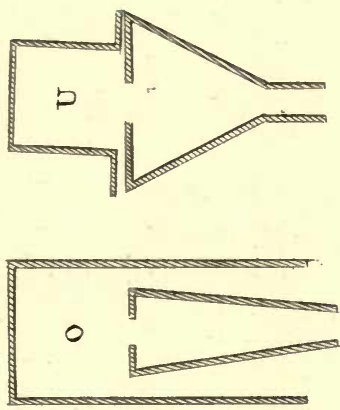

है
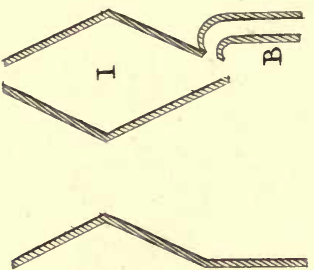

Fi
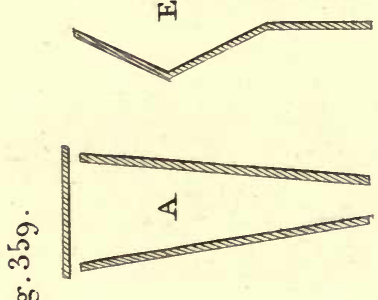

๑

至
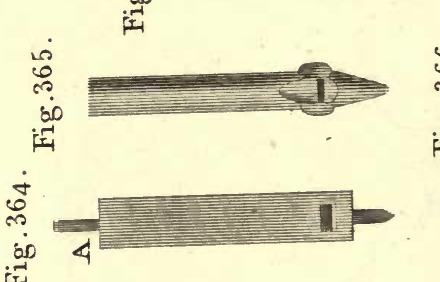
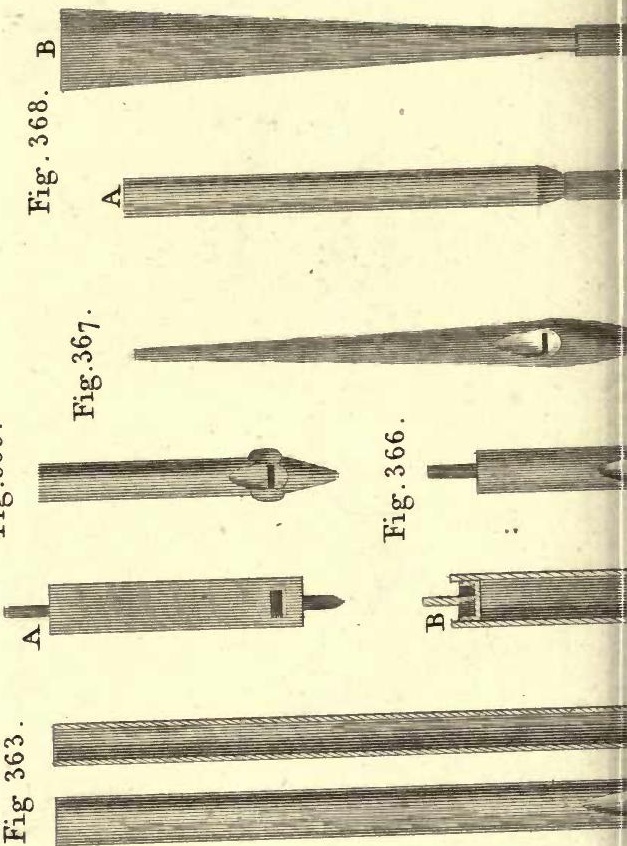

兵 



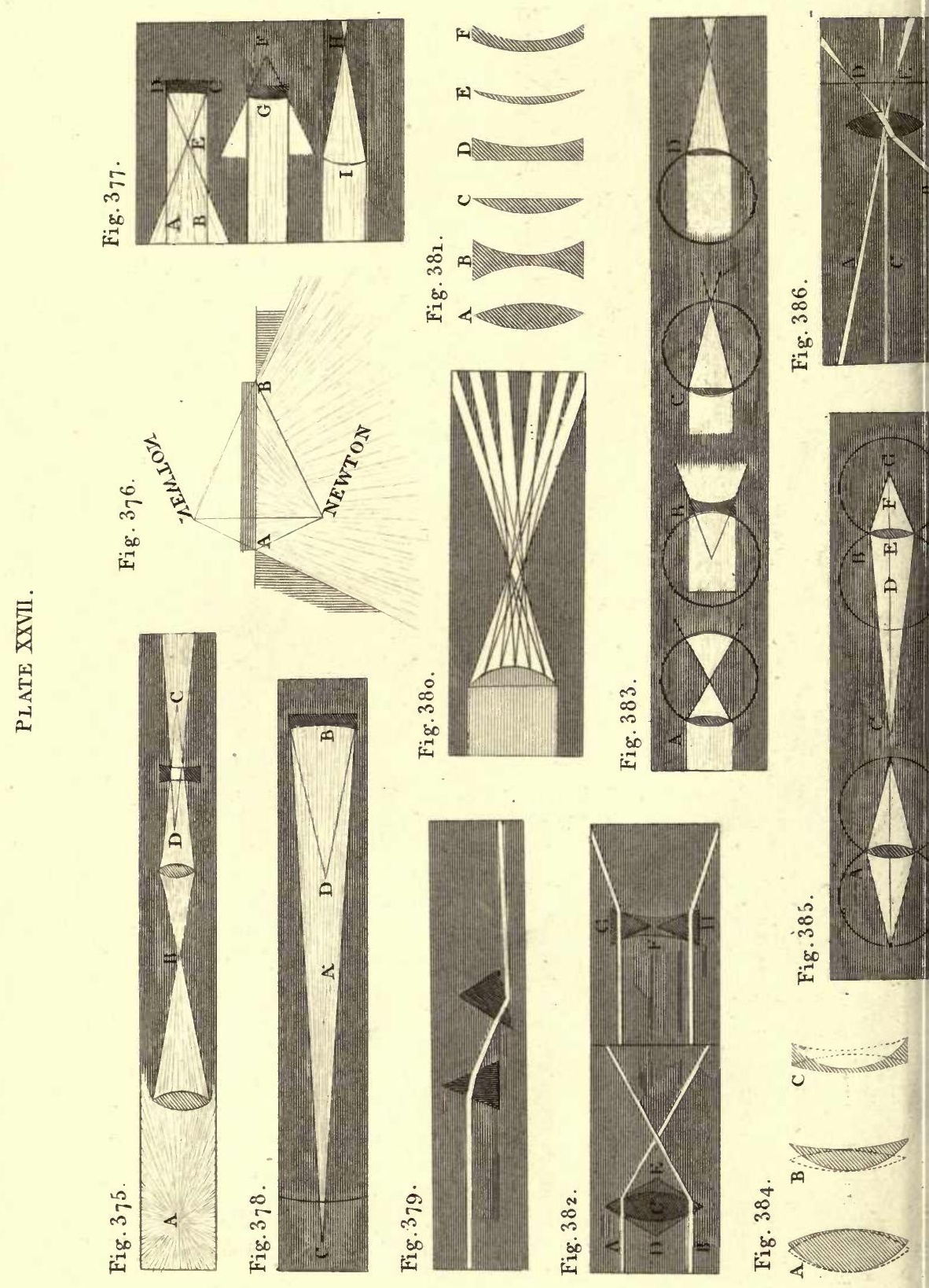




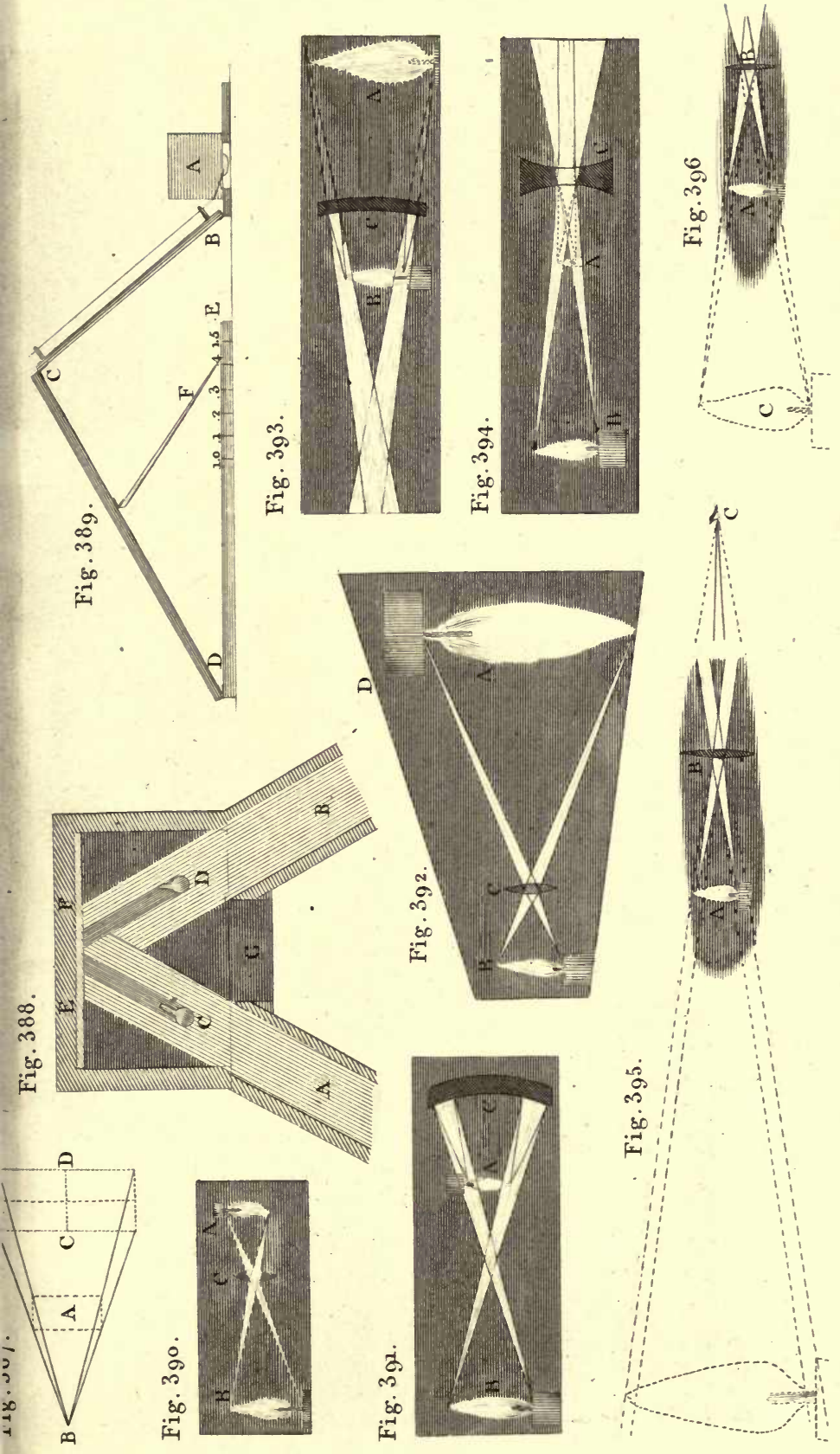





$$
29 \text { Fobsue } 42
$$




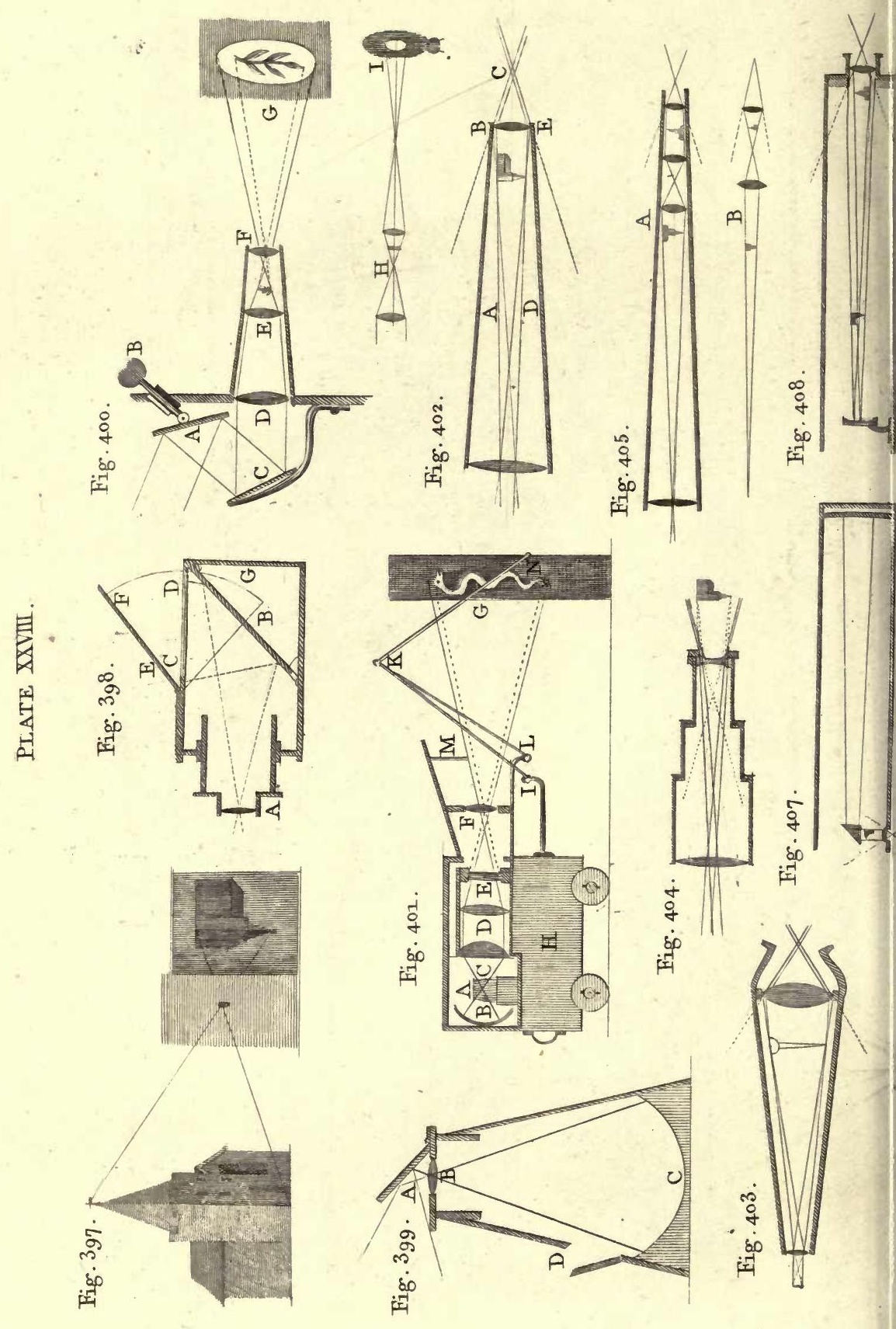




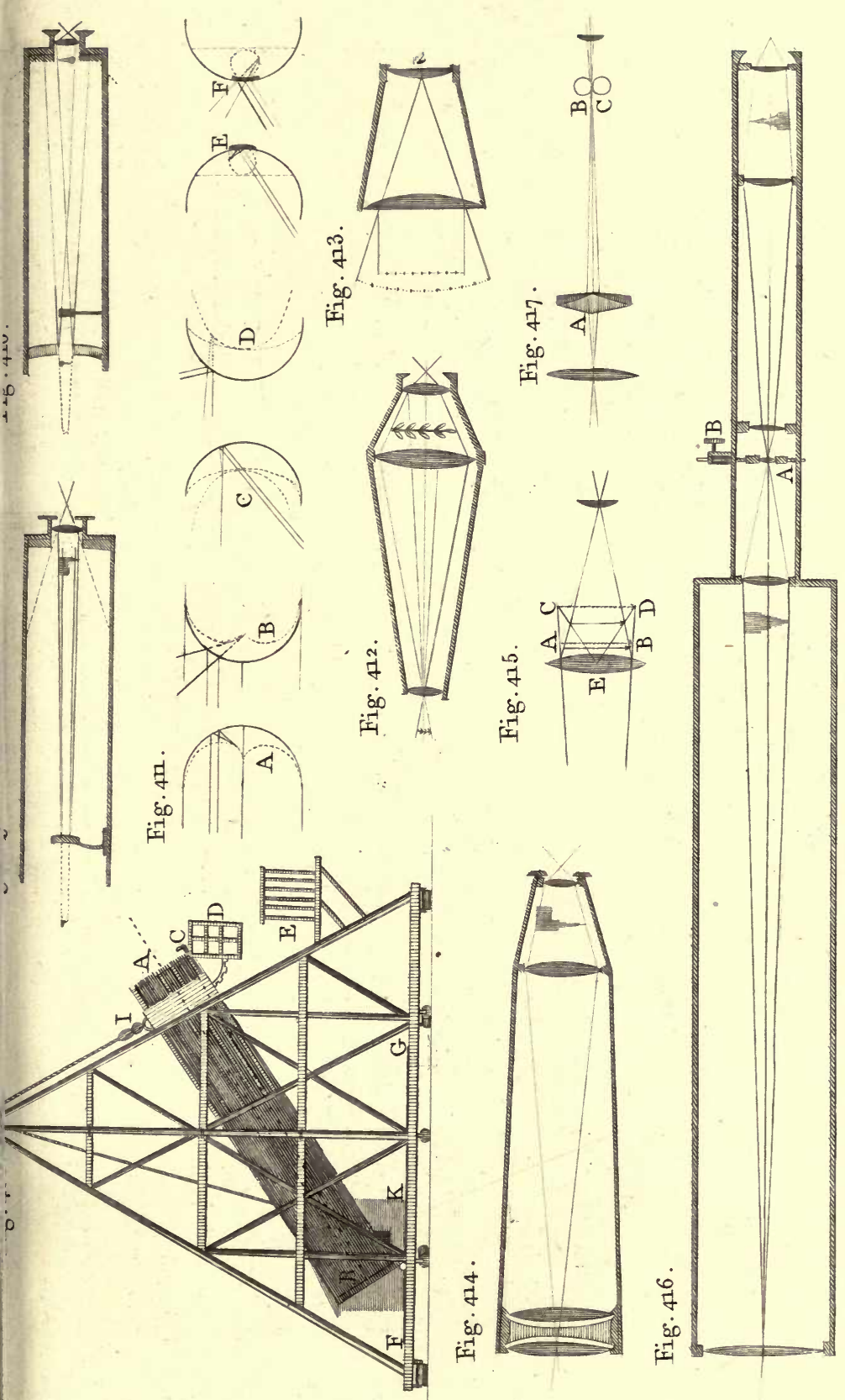



$29+30$

Foktow 41 


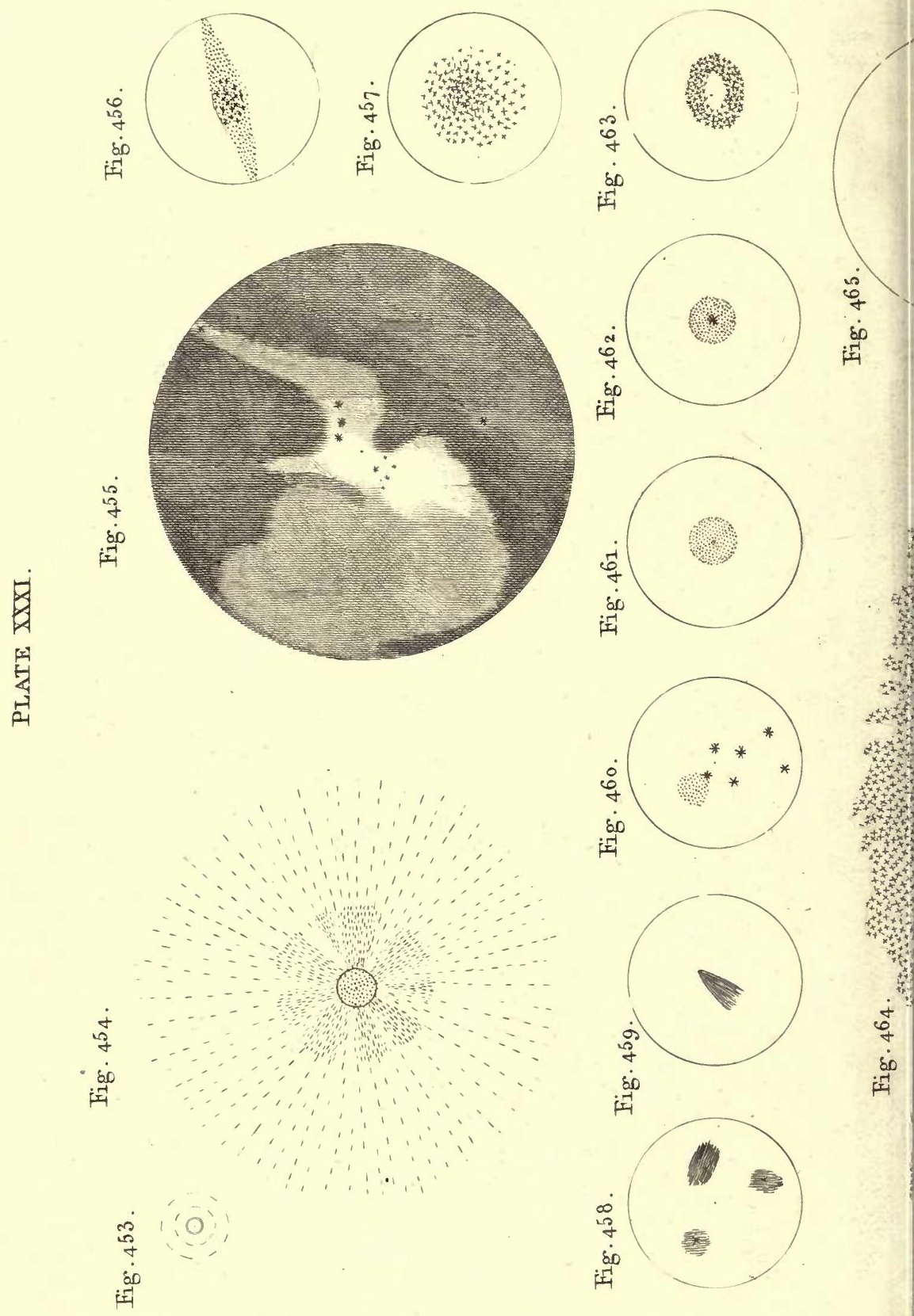




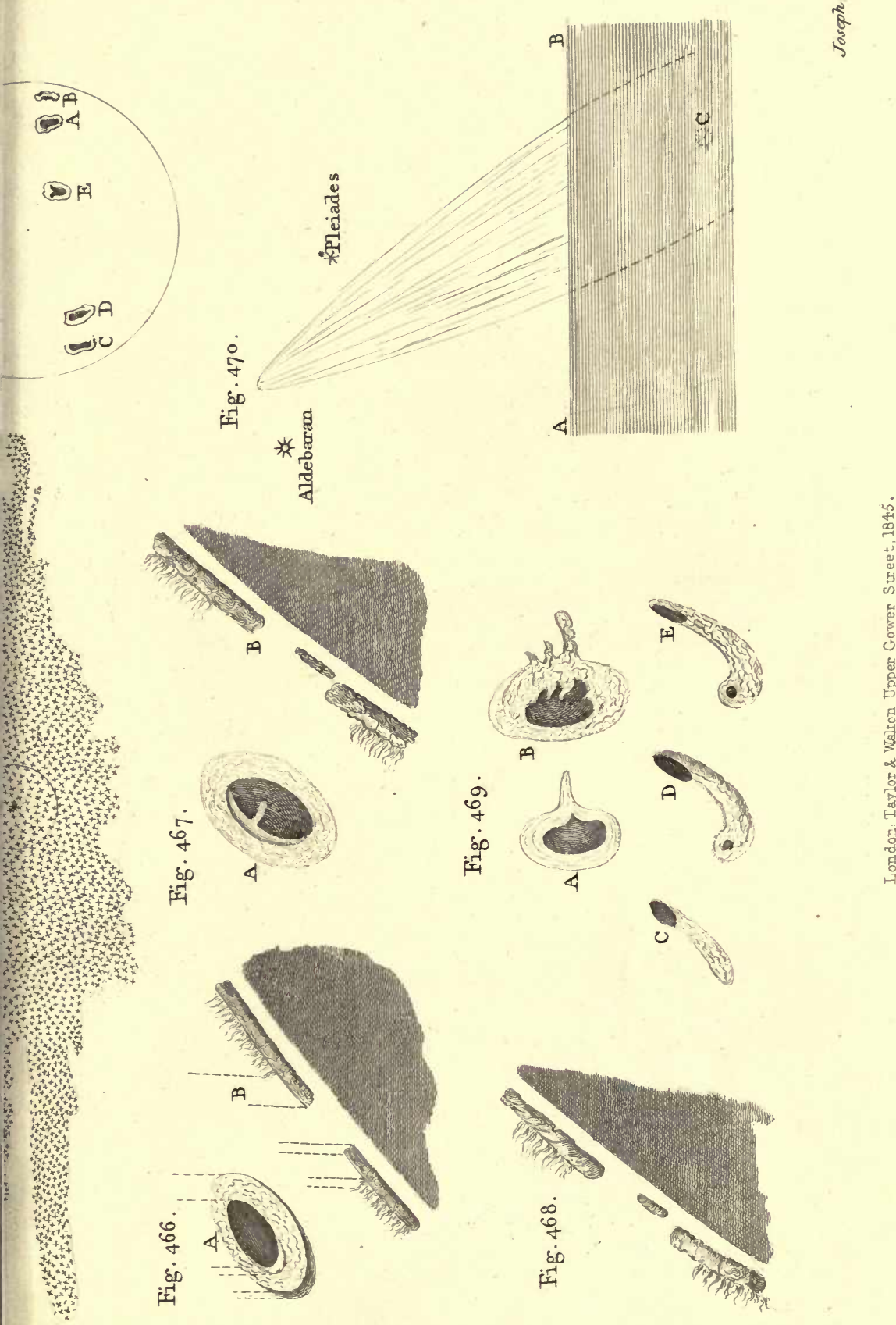





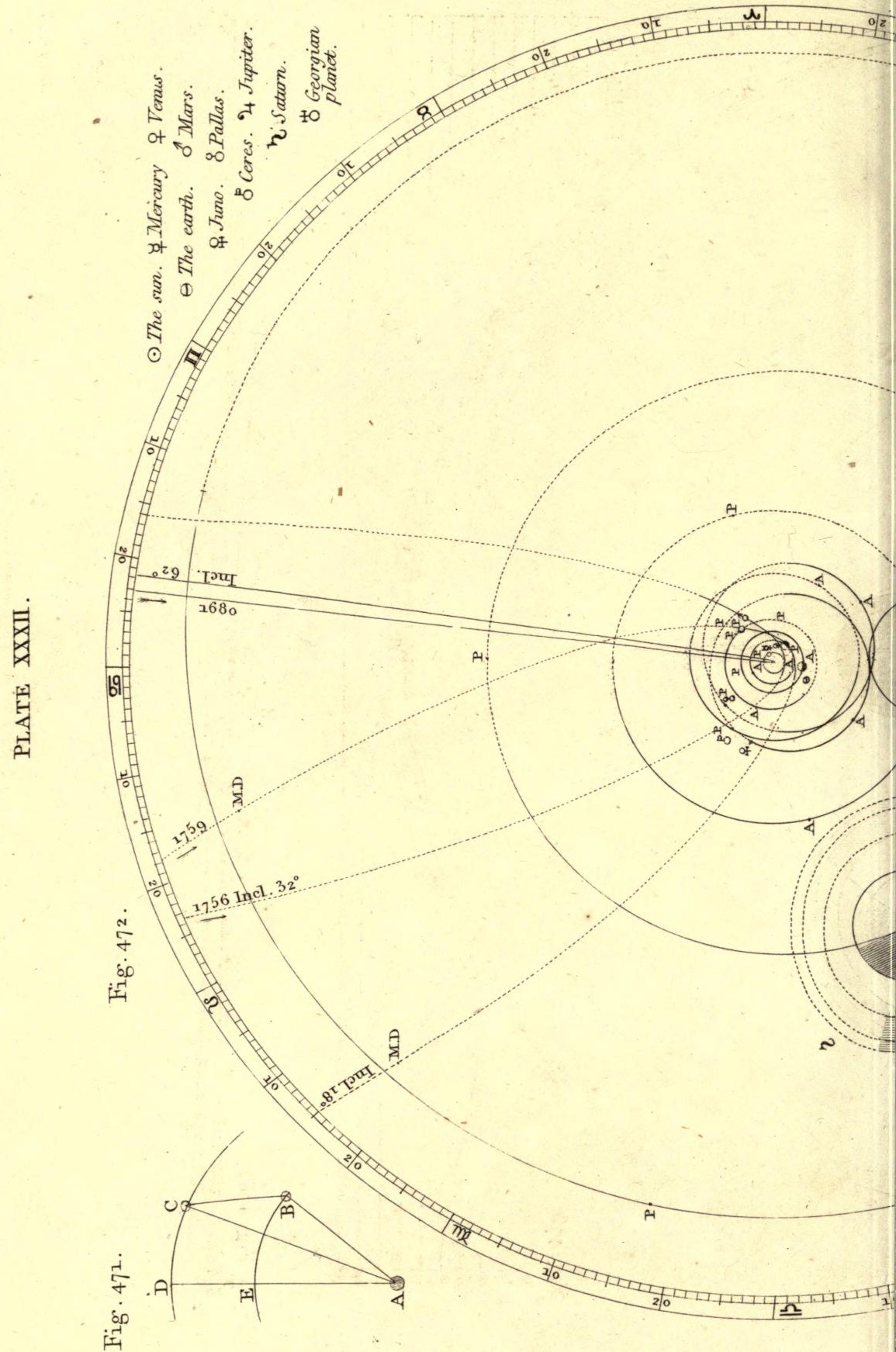





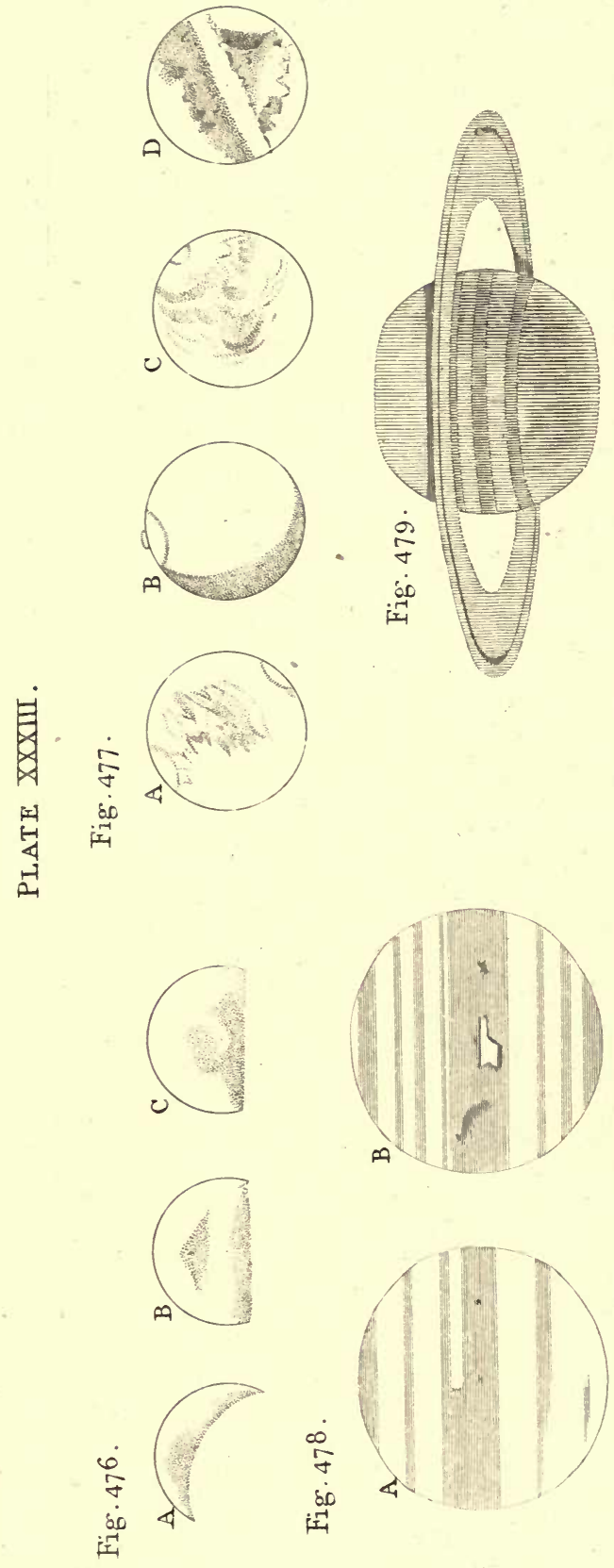

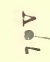




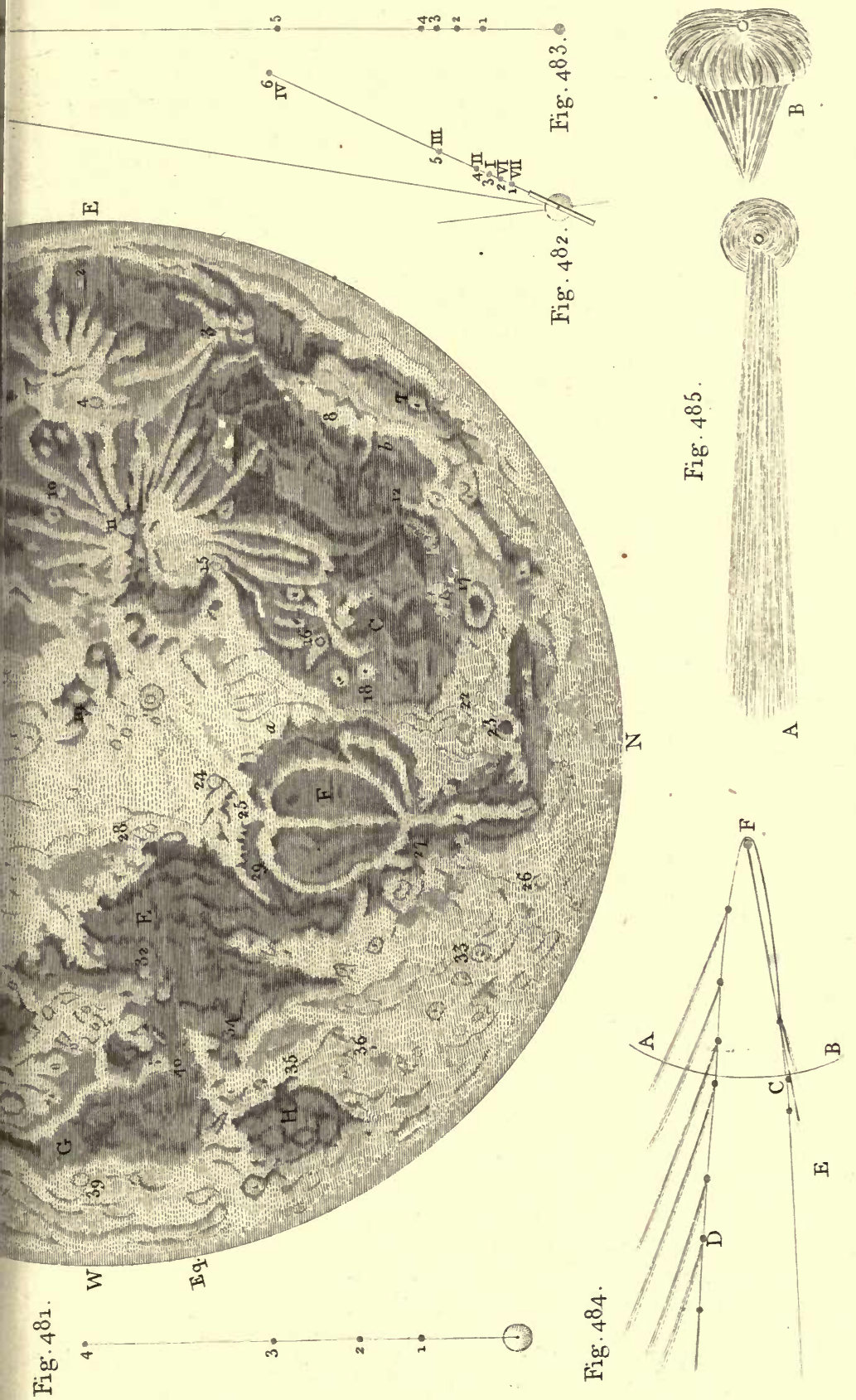

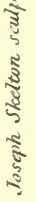





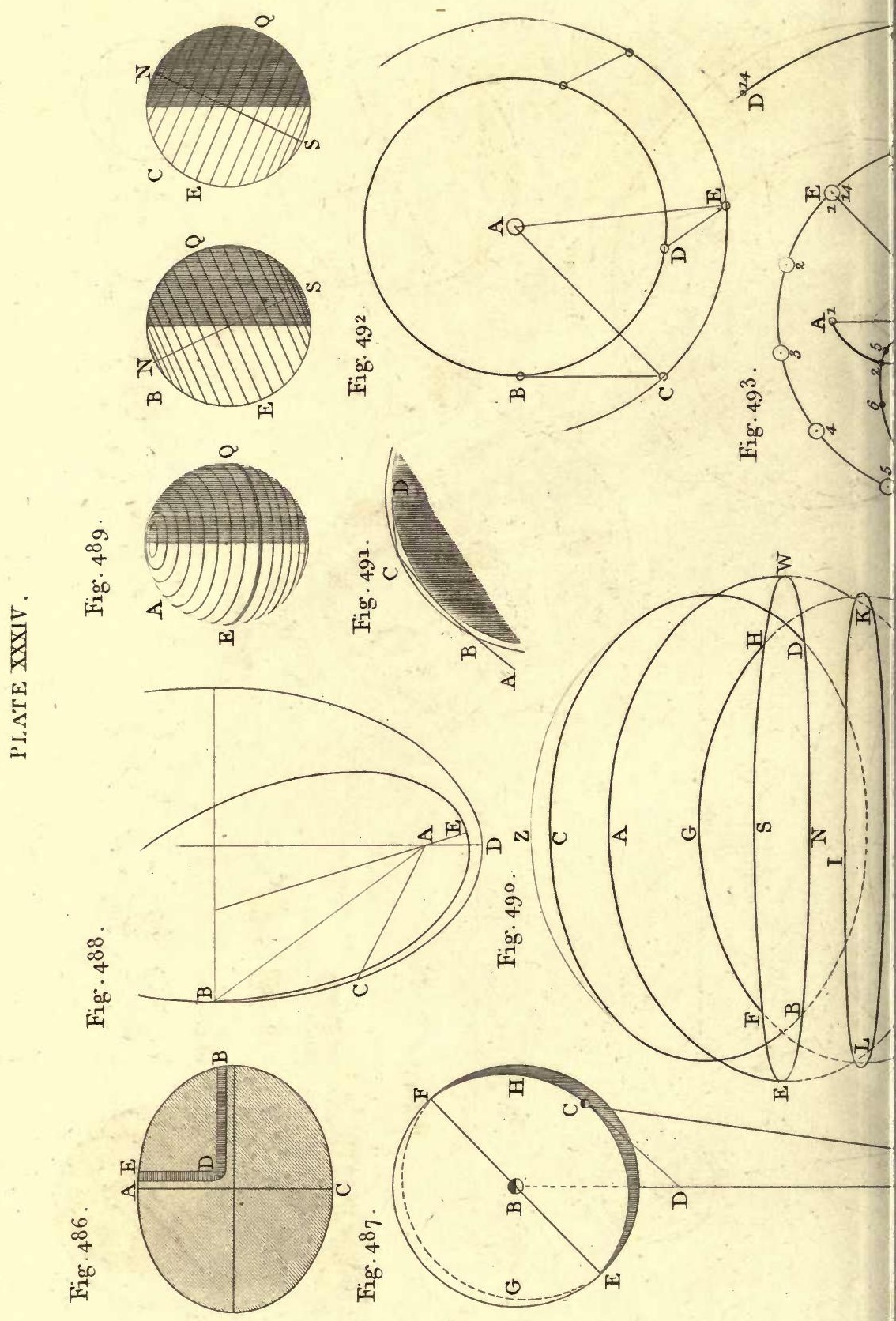




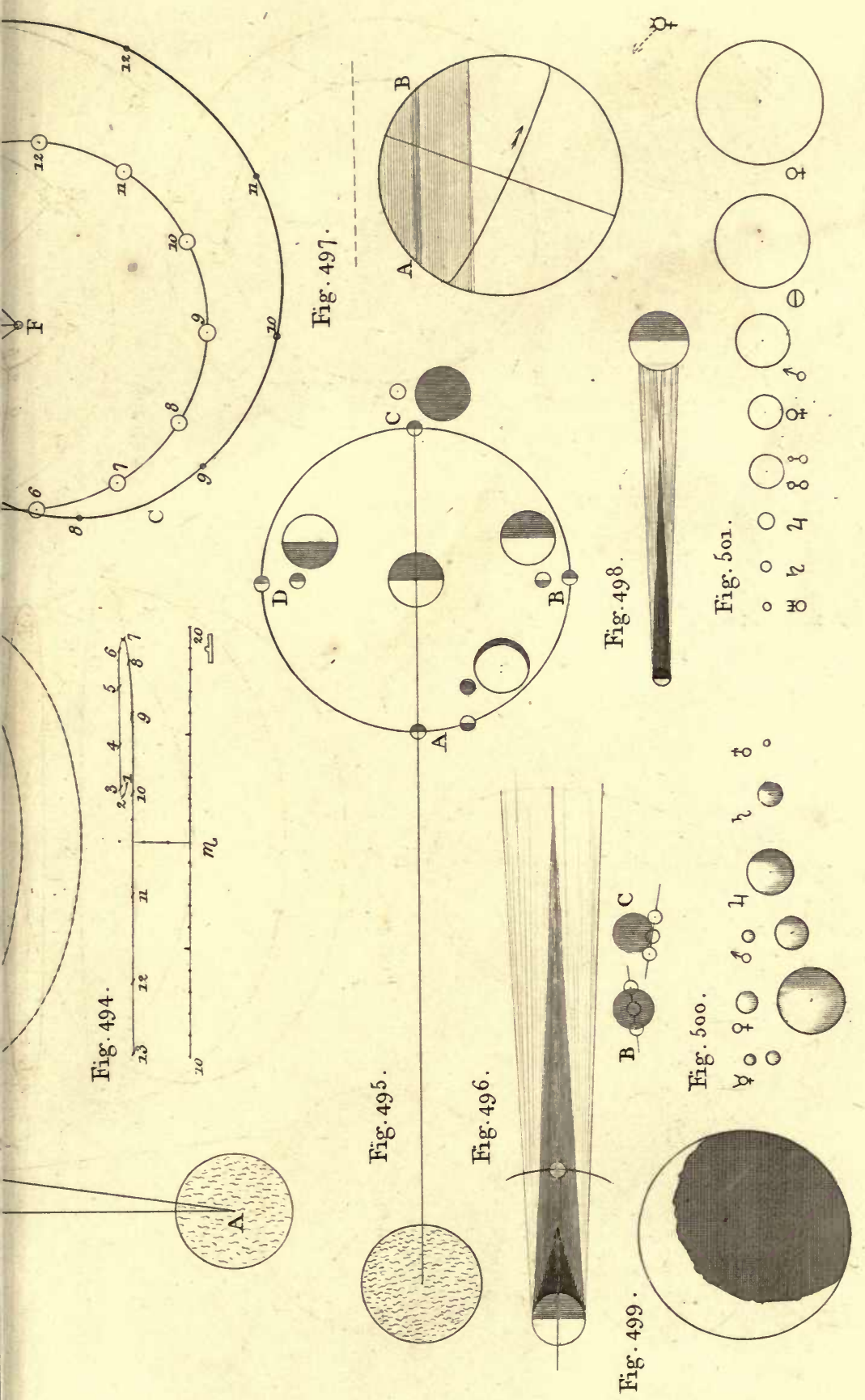






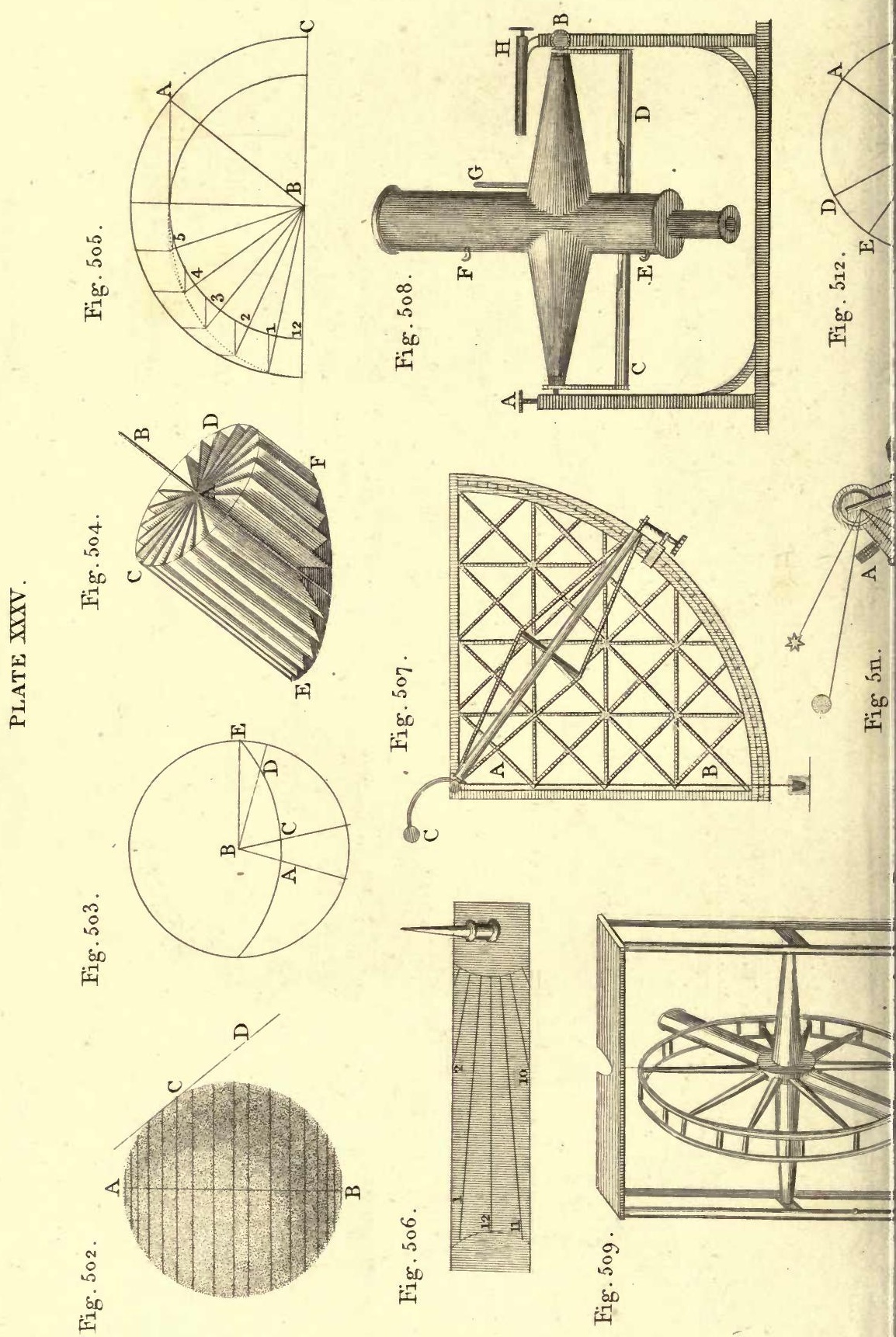




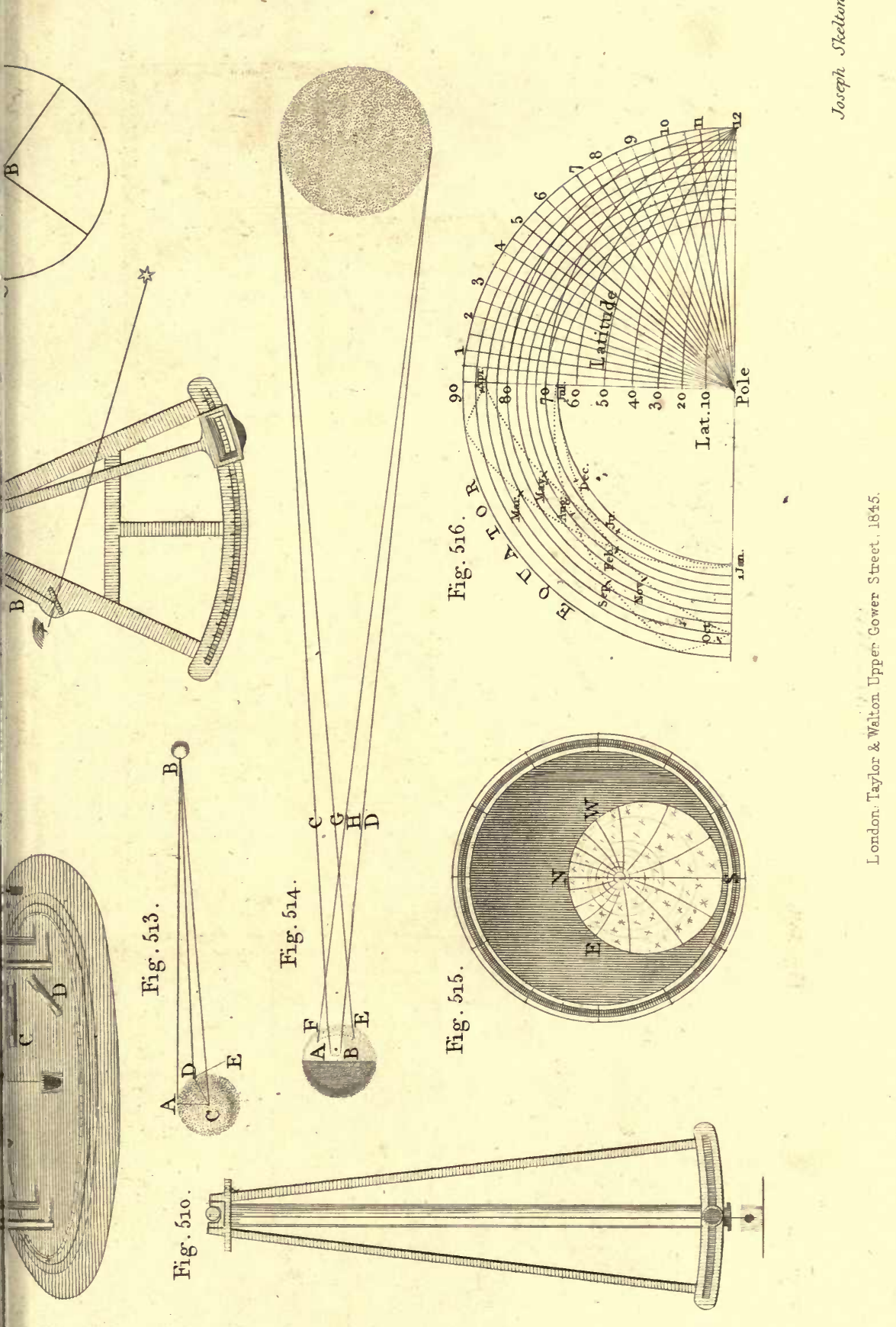





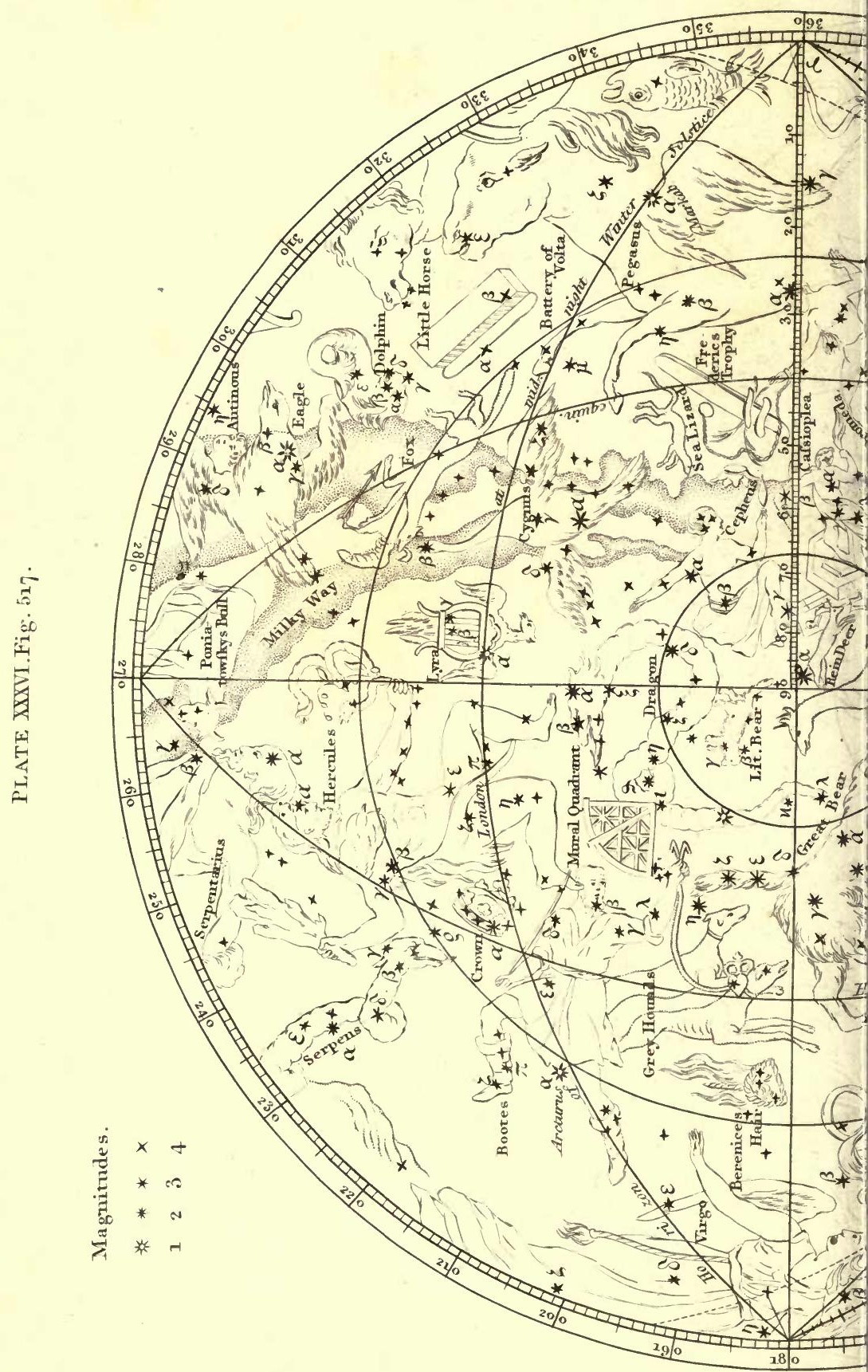





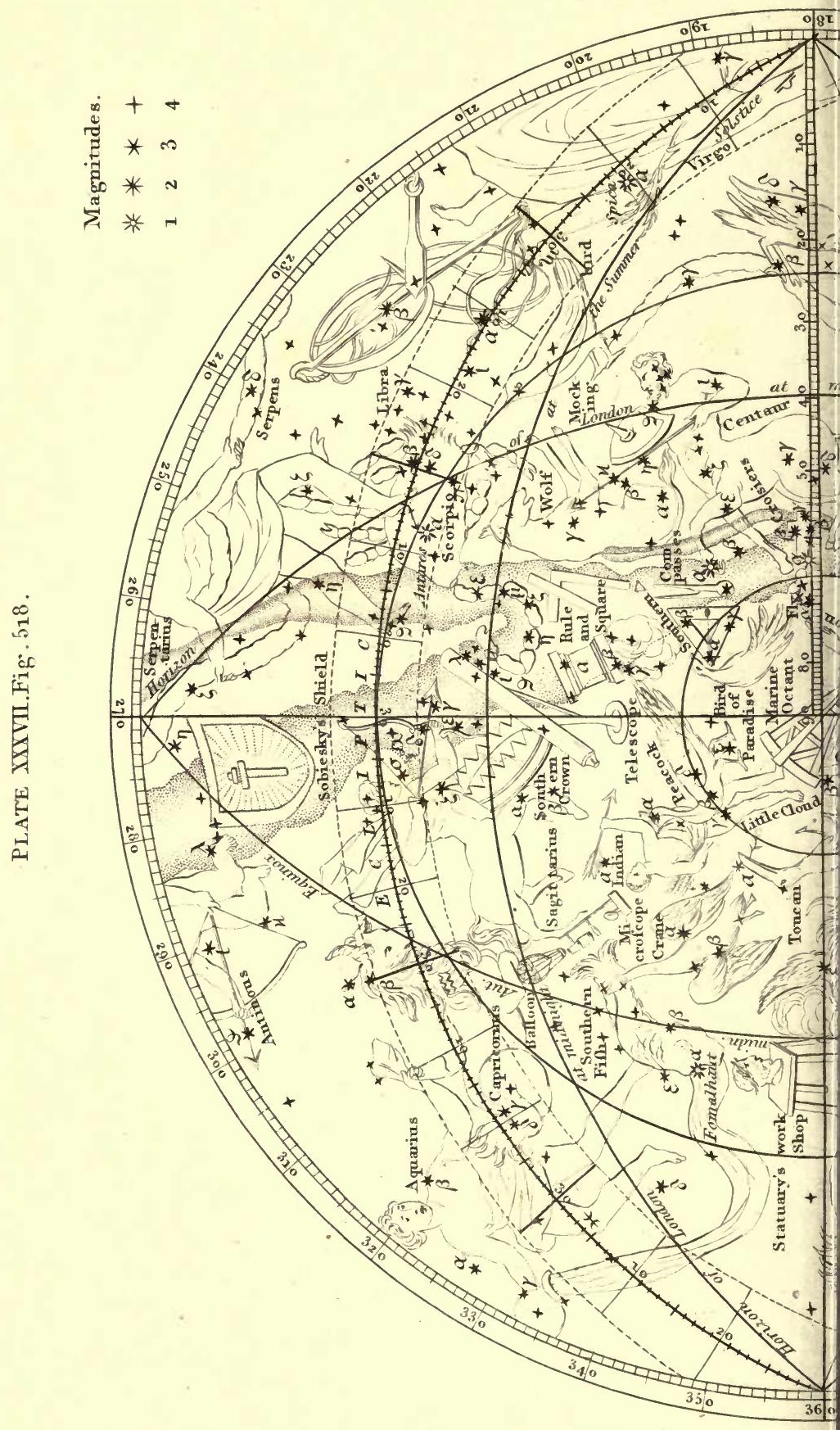




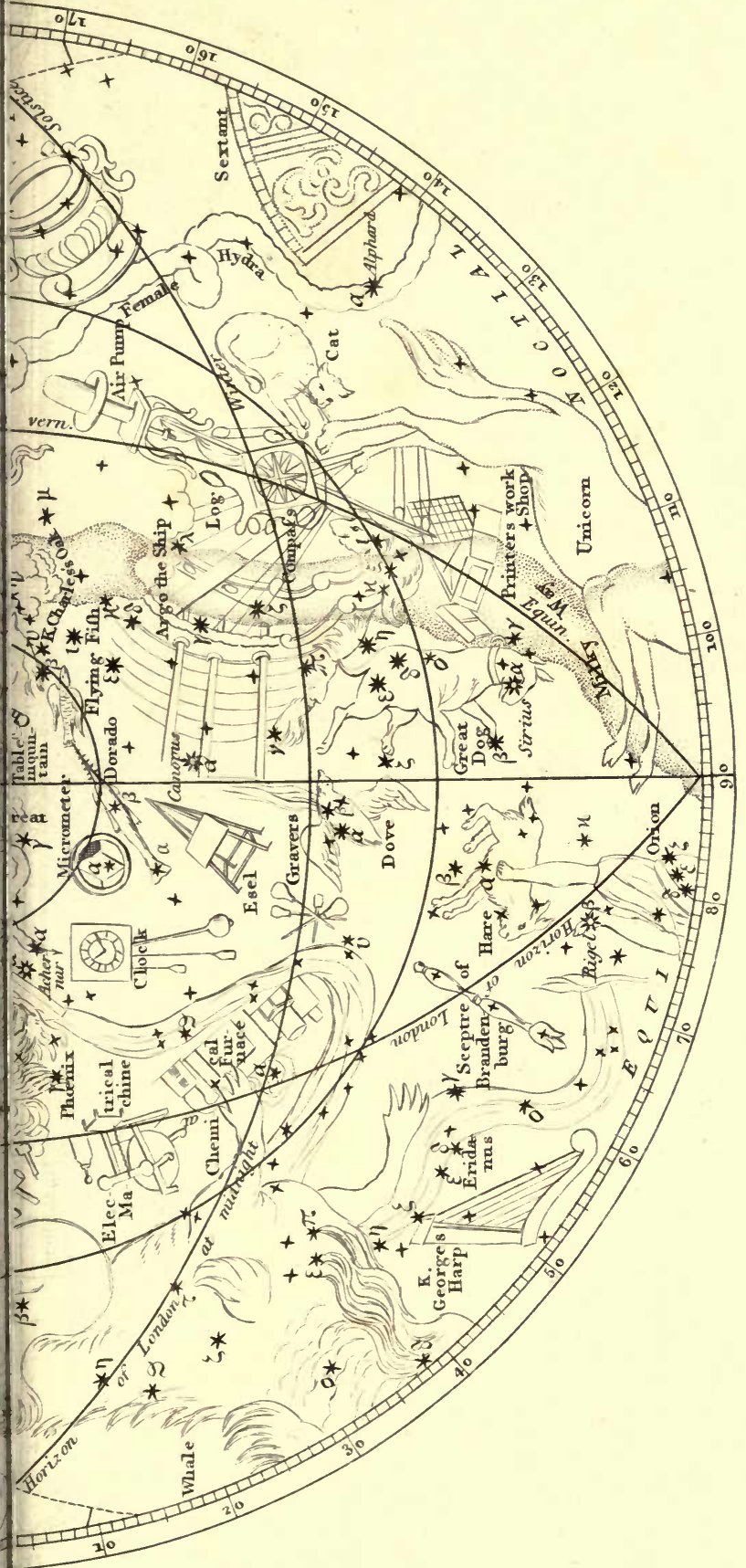




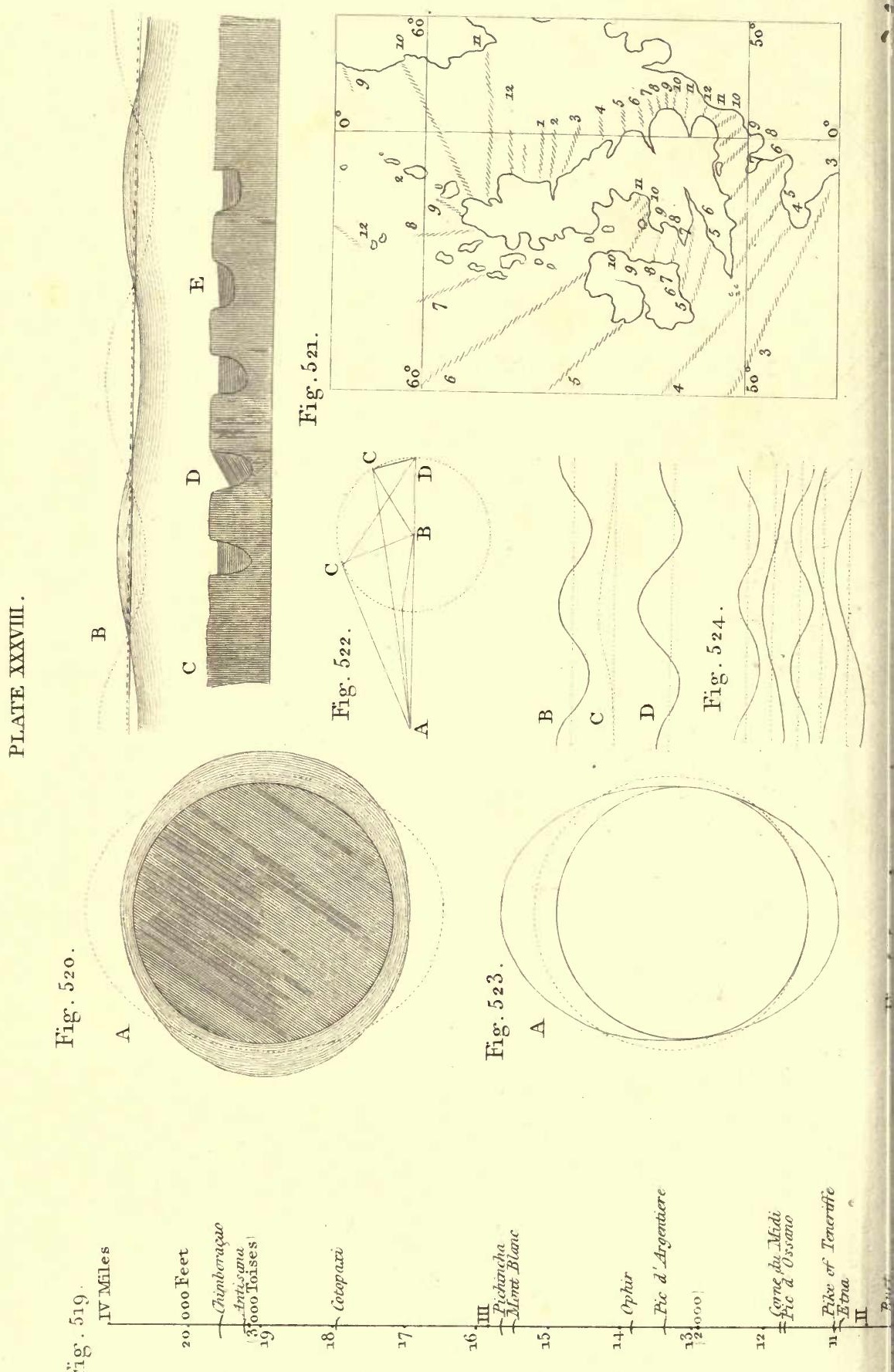



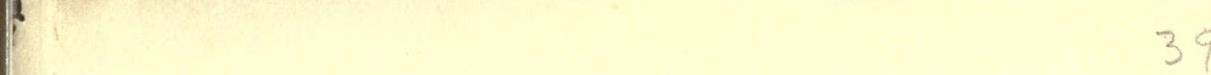




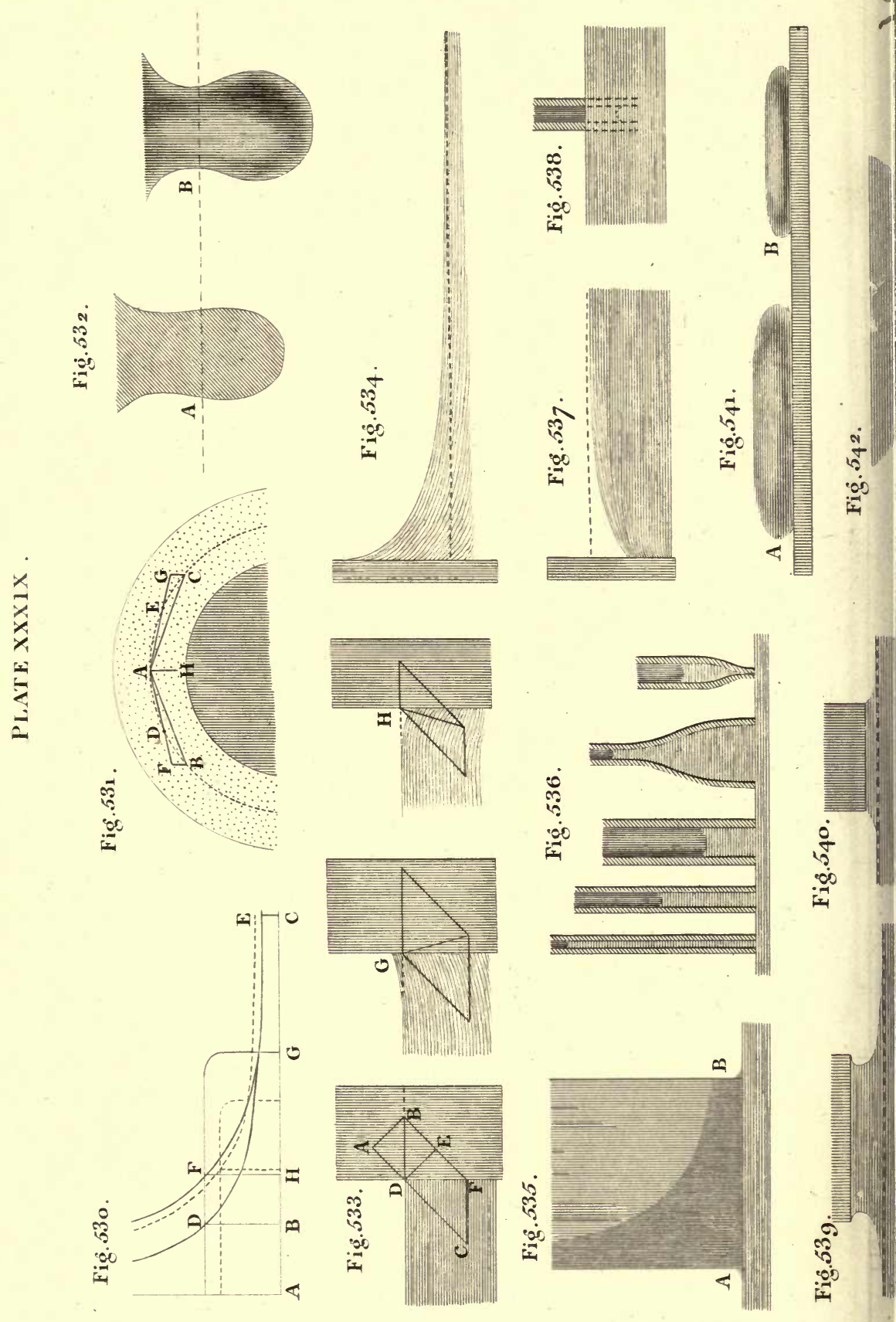



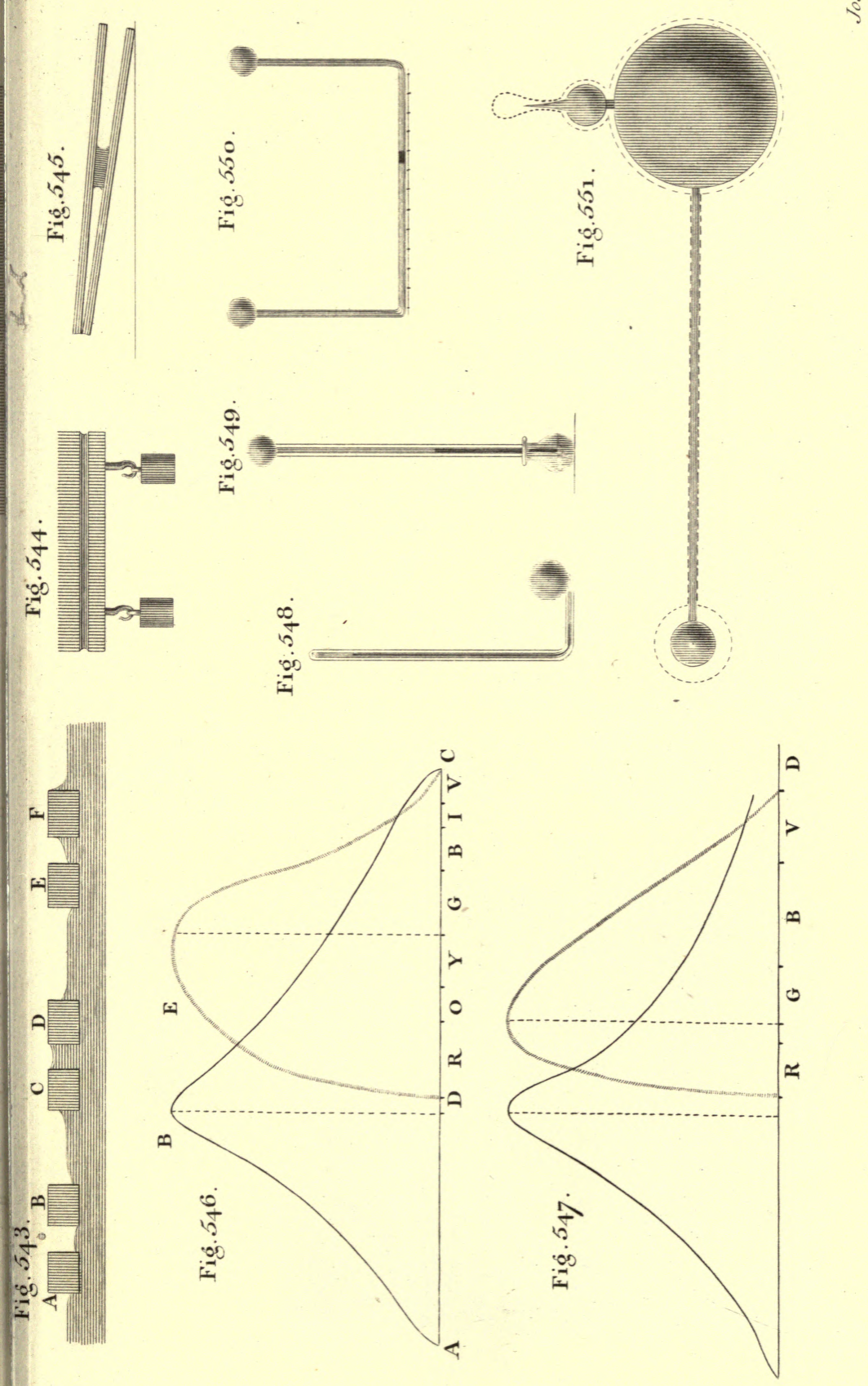



$$
40
$$




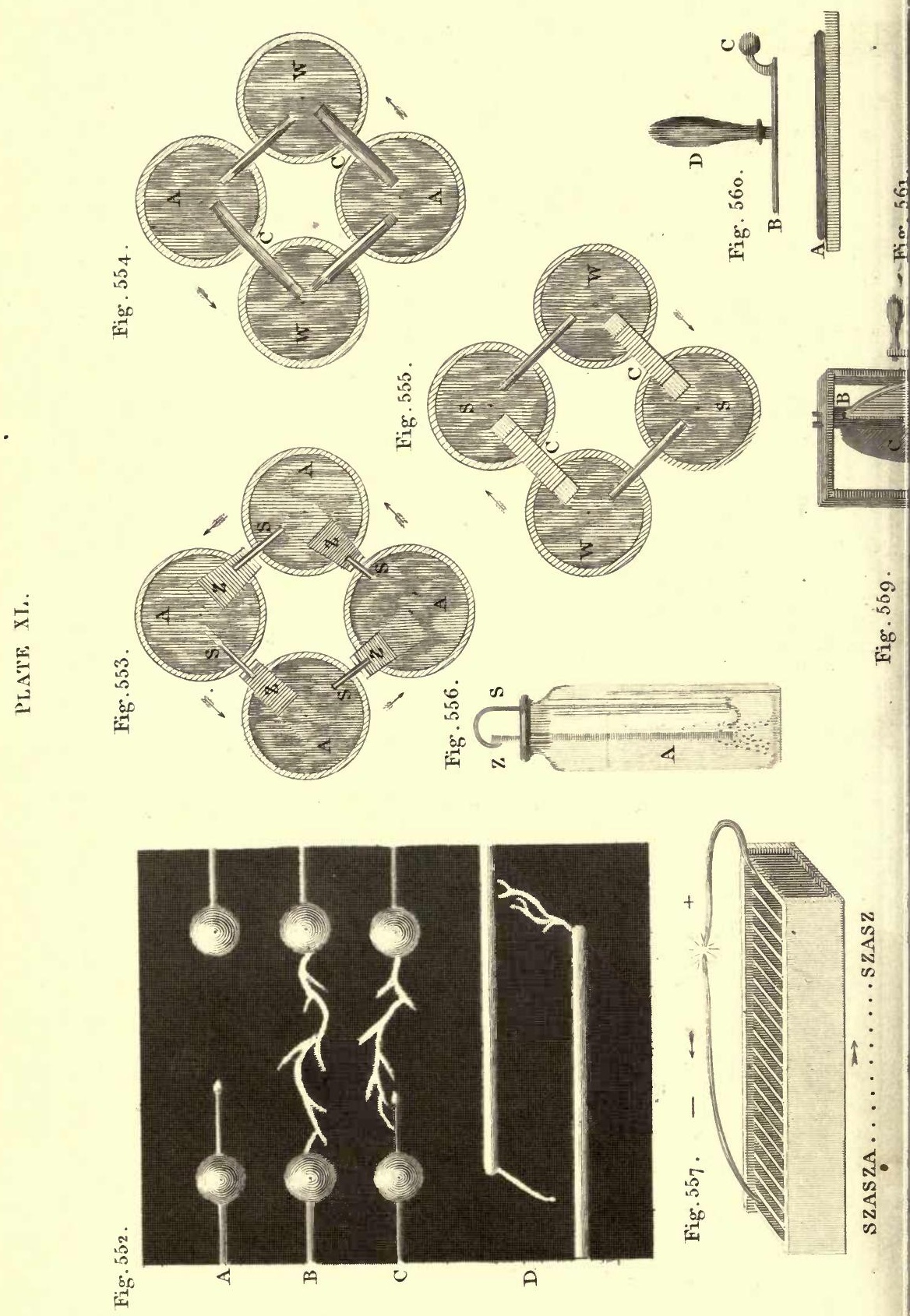




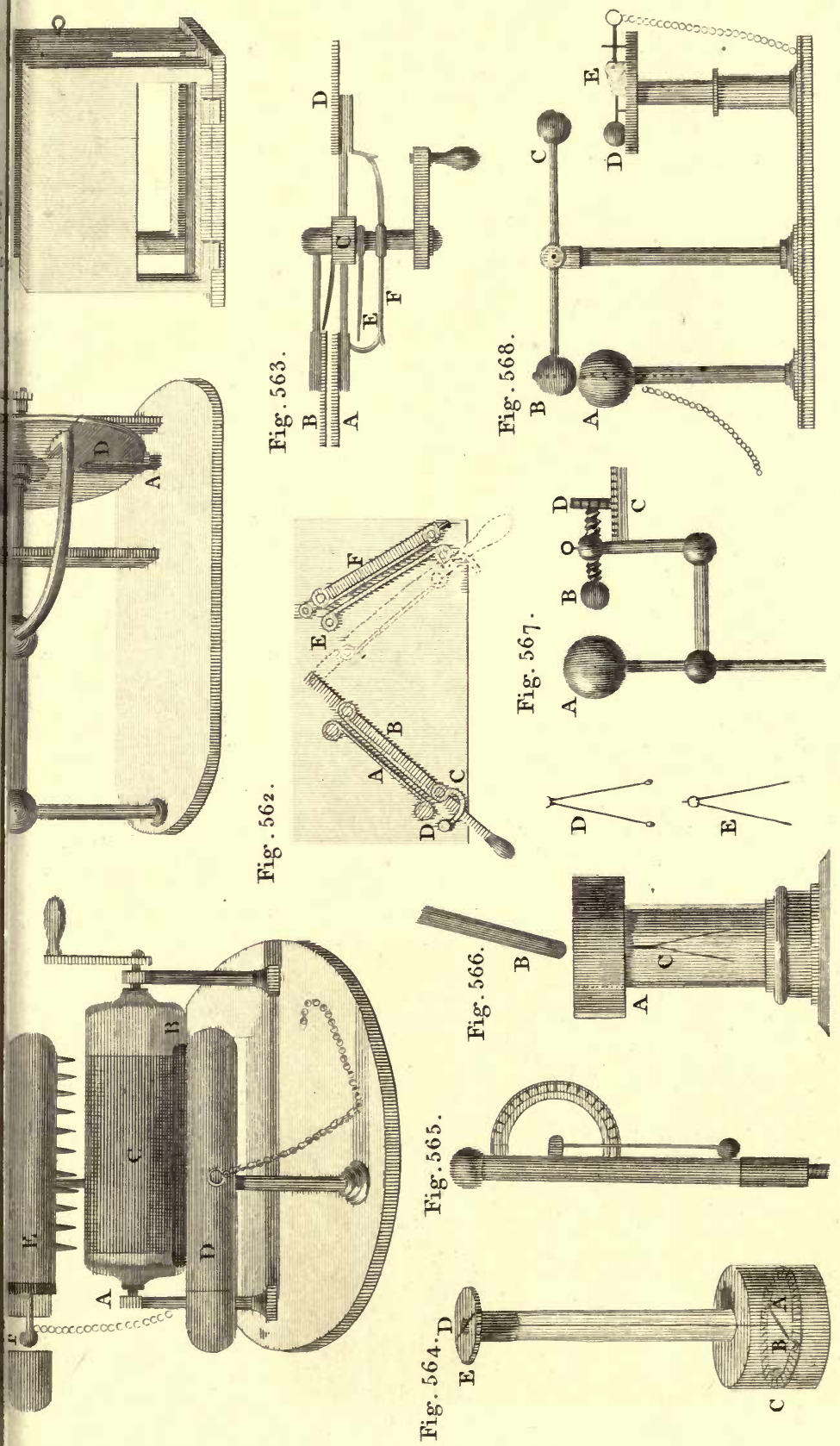




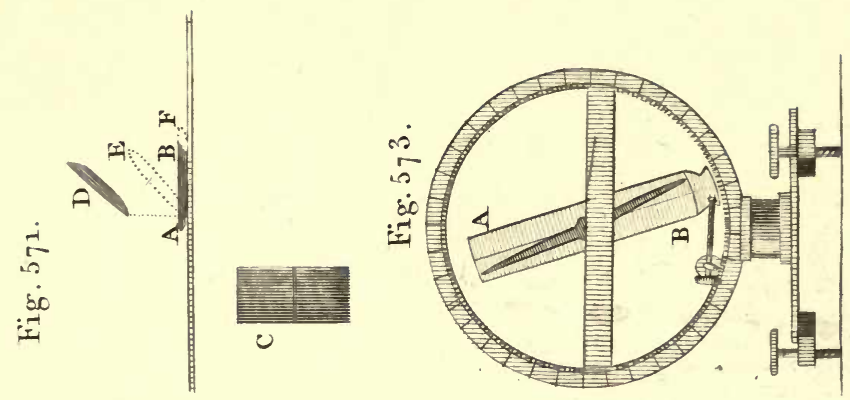

言
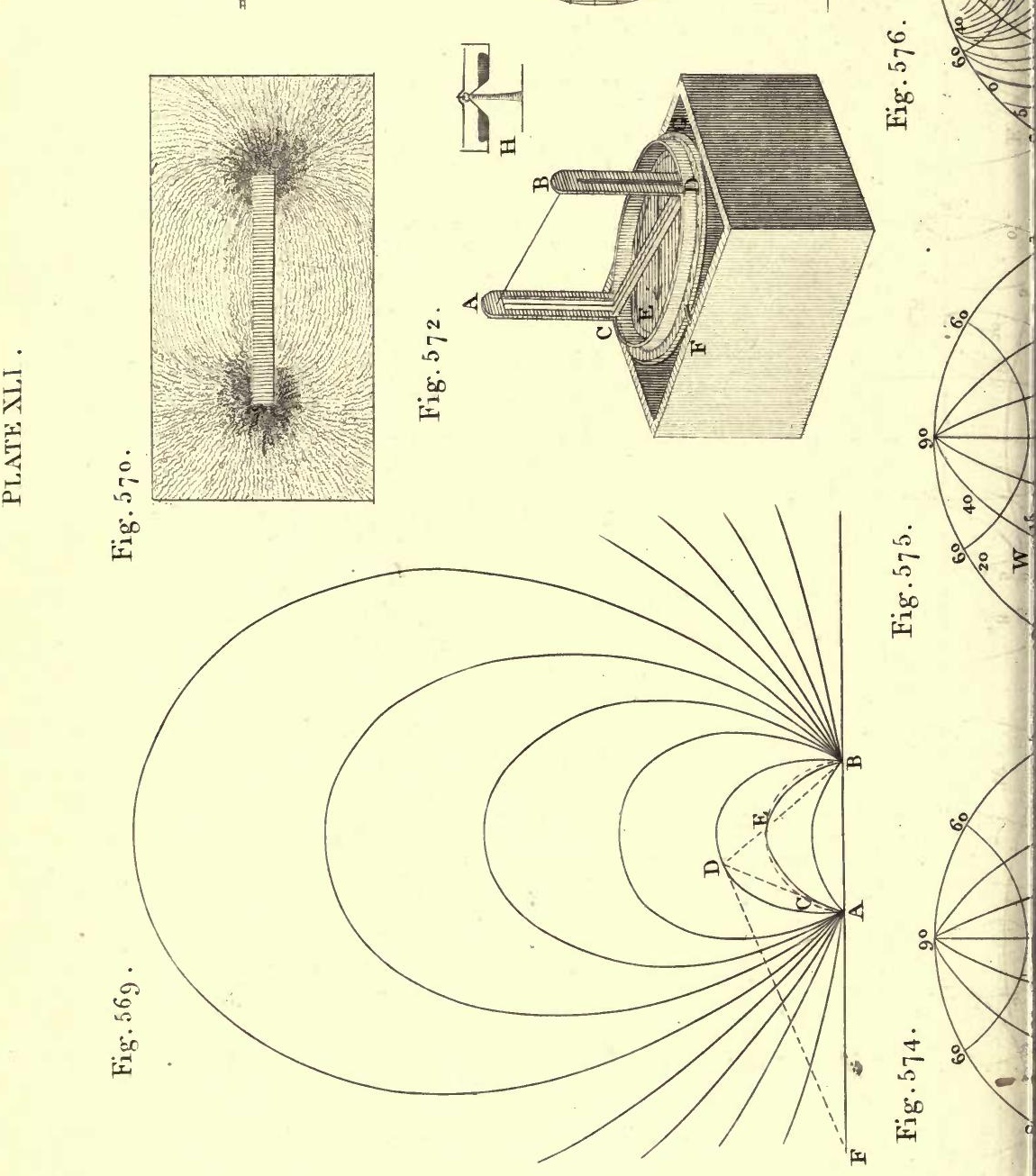


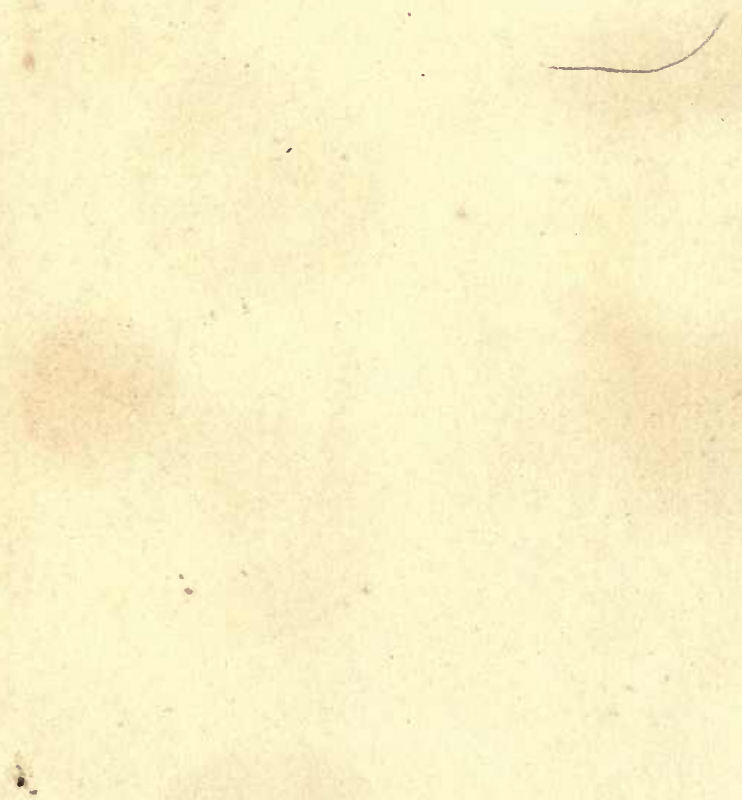


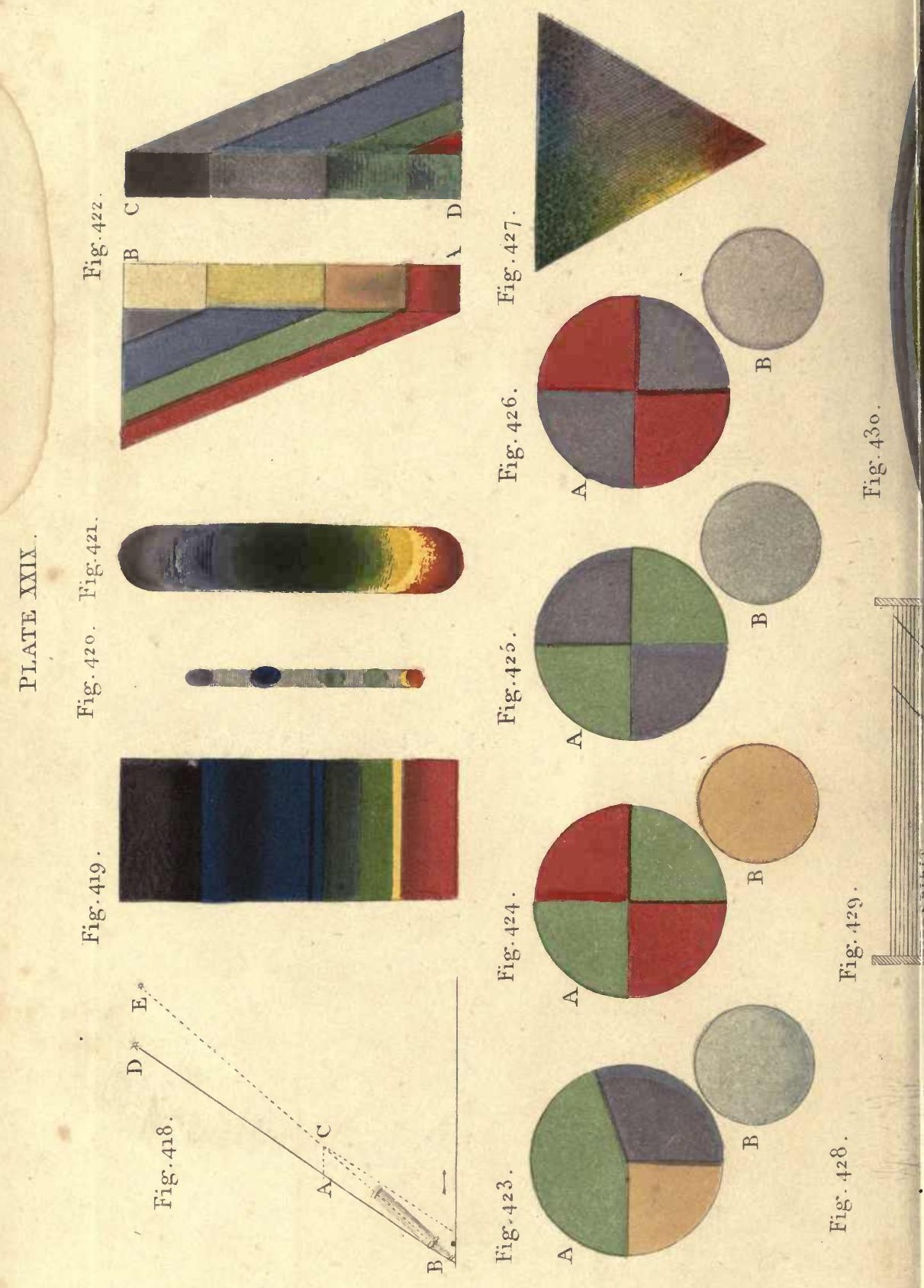




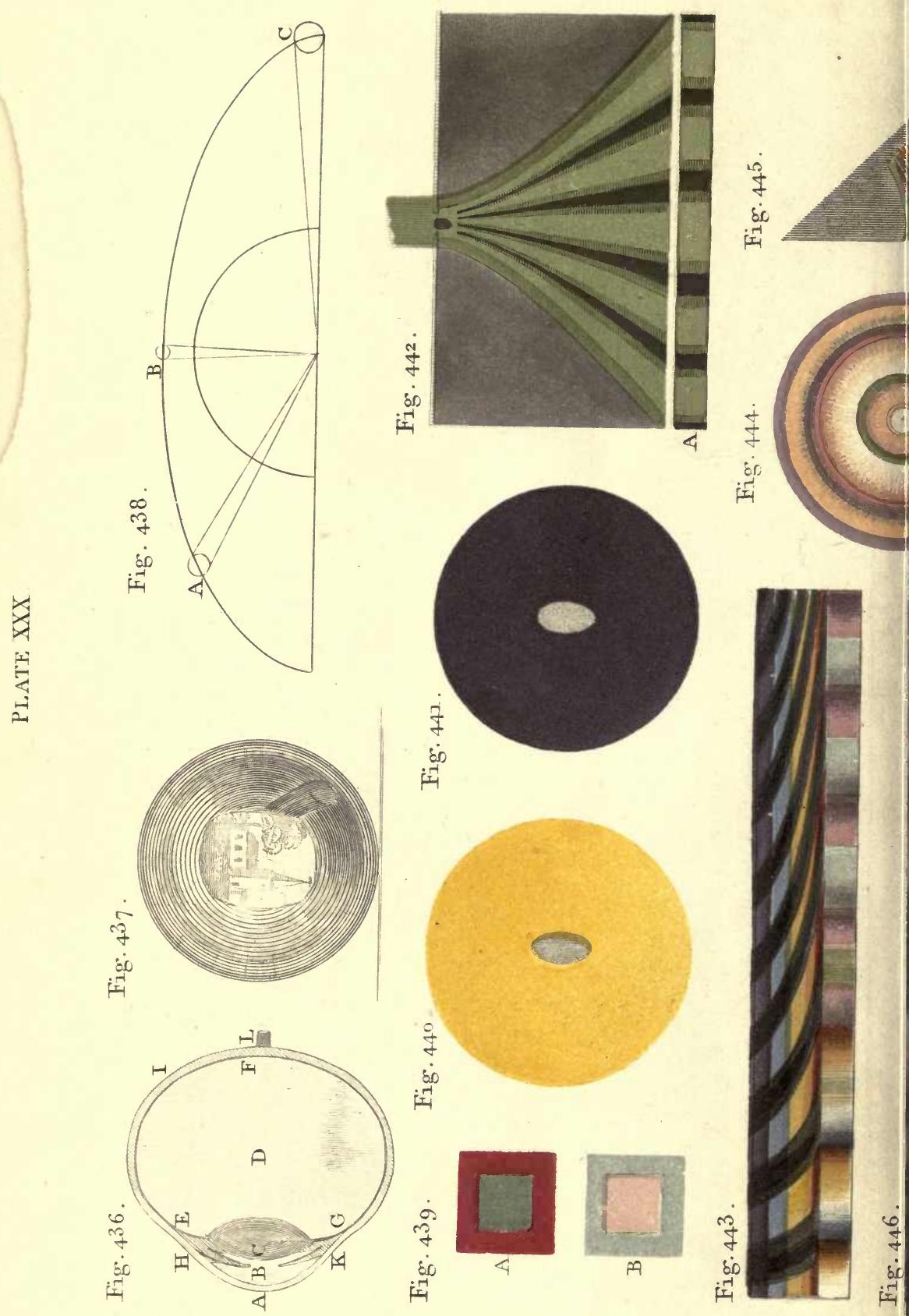


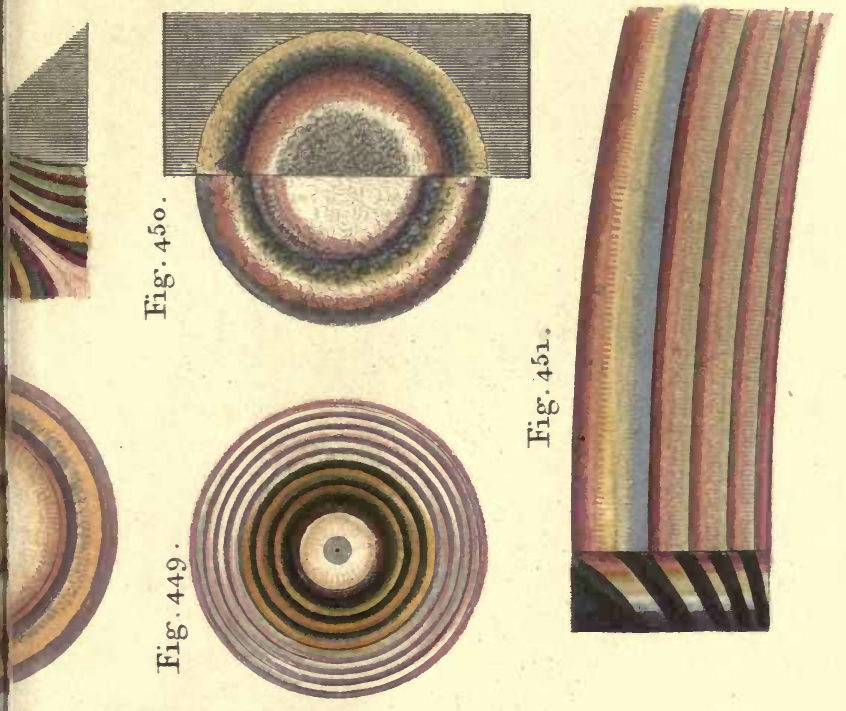

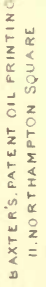
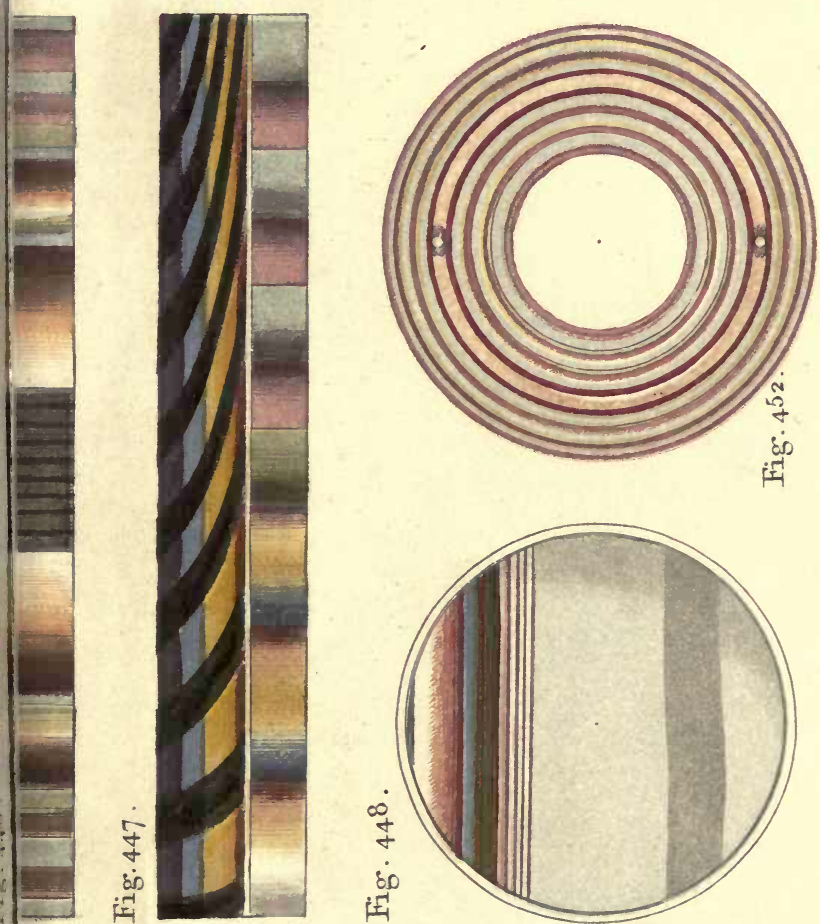

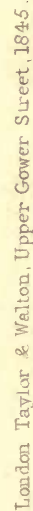

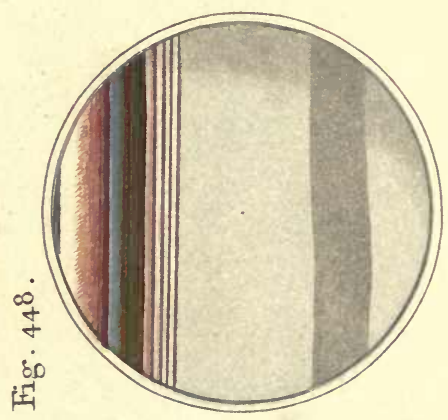





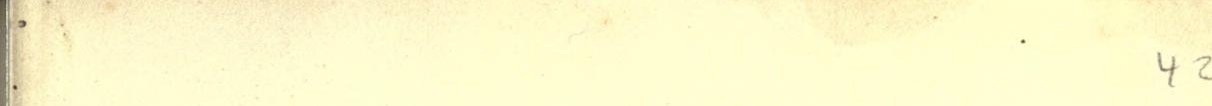




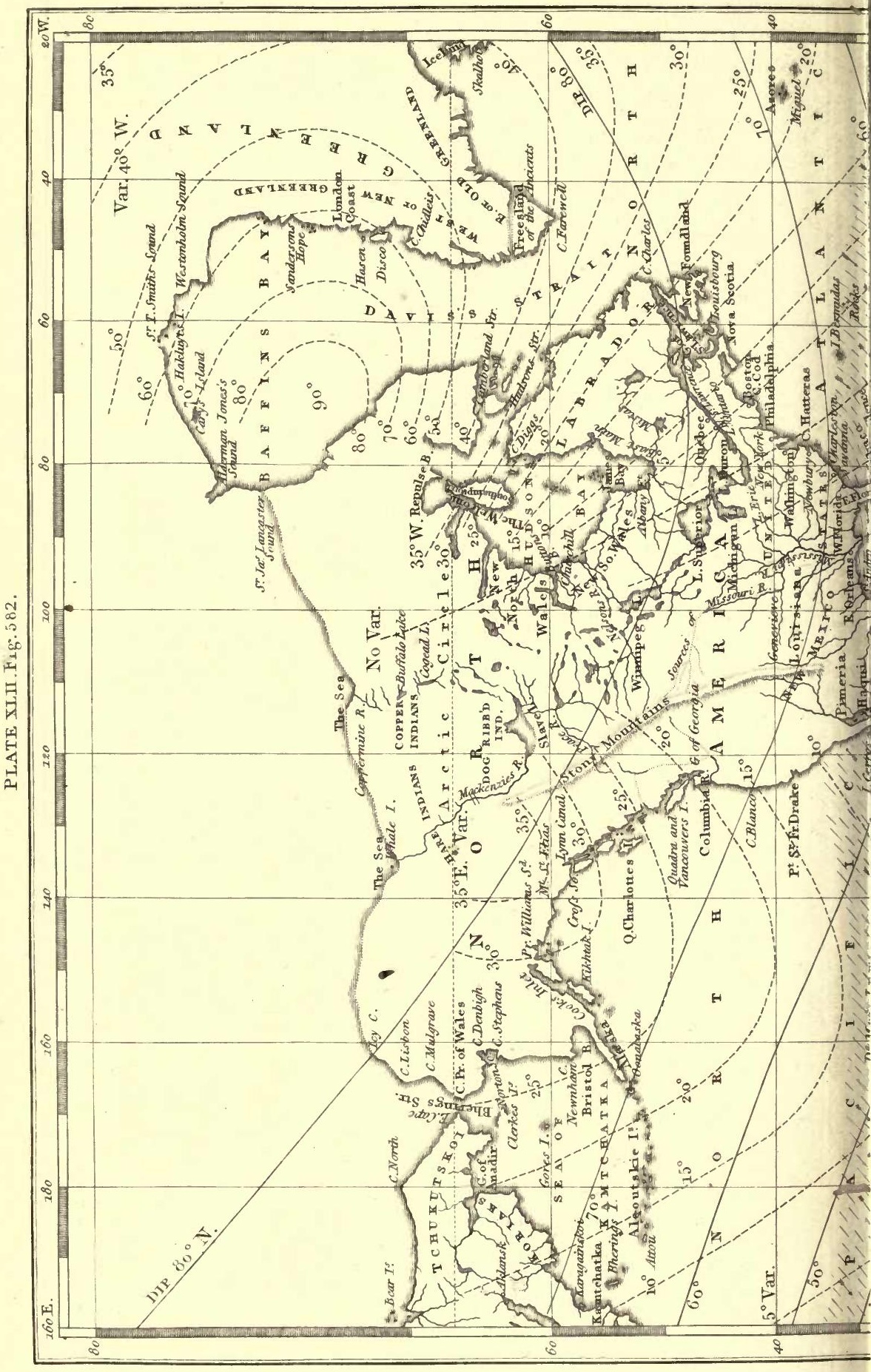




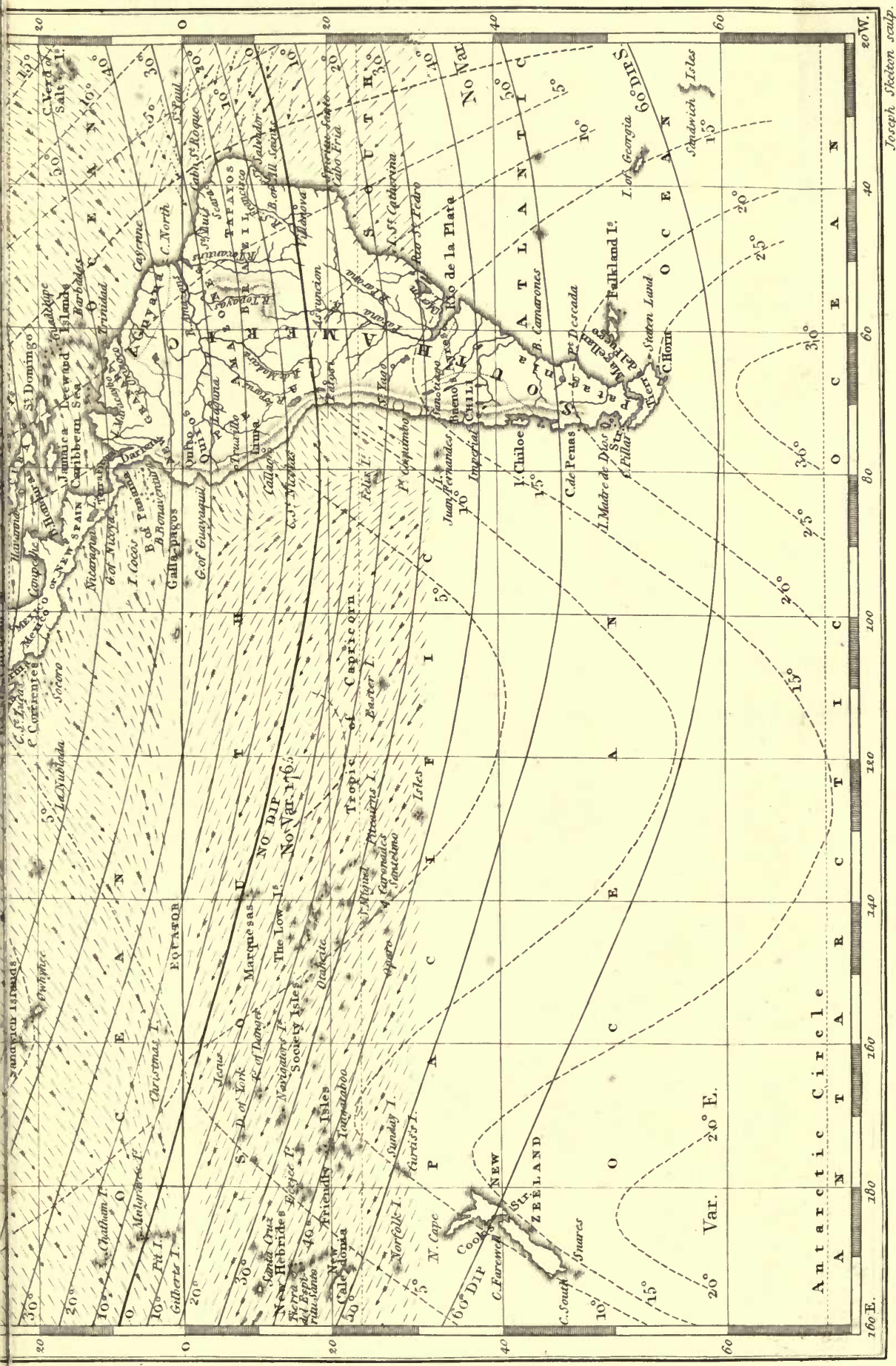





$$
43
$$




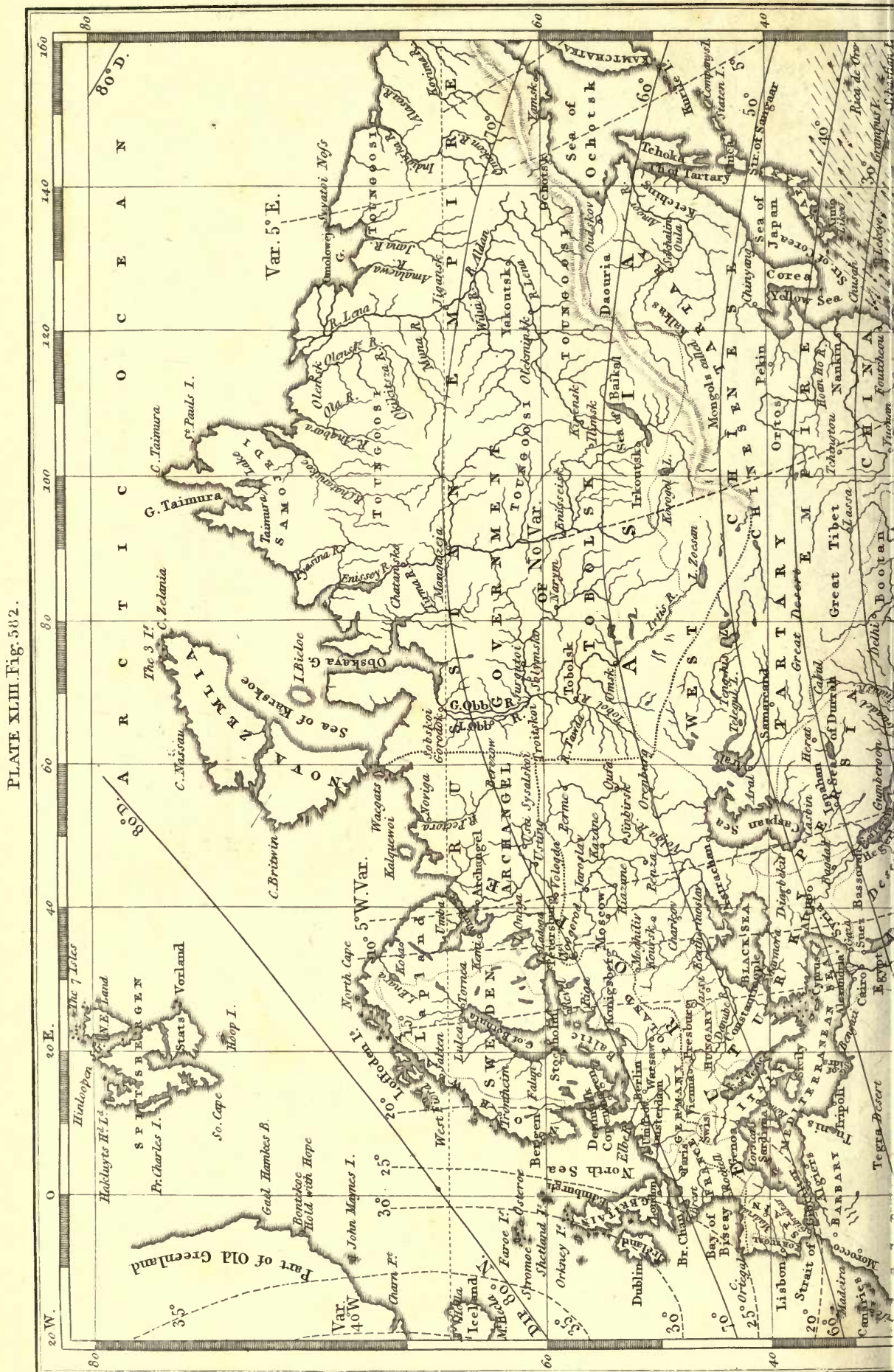








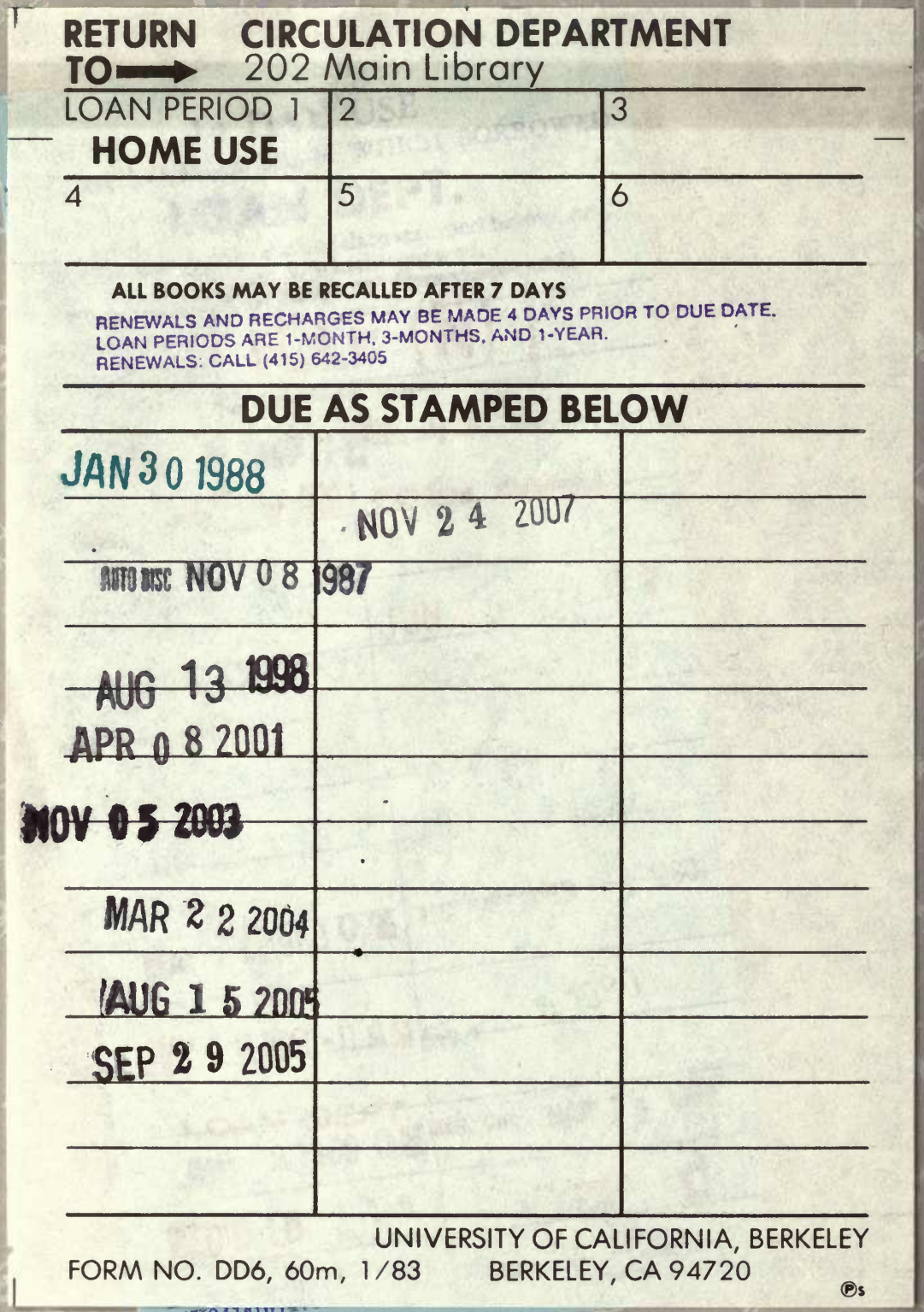




\section{YC 22701}

\section{U.C. BERKELEY LIBRARIES ||||||||||||||||||||||||||||||||||||||||||| C004366489}
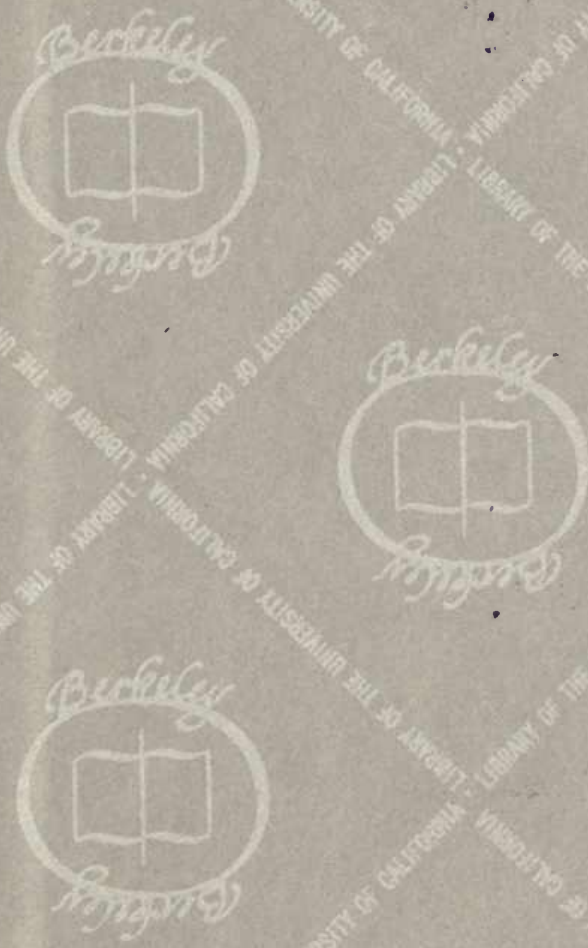
